\title{
Characterization of FBXO7 (PARK15) knockout mice modeling Parkinsonian-Pyramidal Syndrome
}

\author{
Dissertation \\ for the award of the degree \\ "Doctor rerum naturalium" \\ of the Georg-August-Universität Göttingen \\ within the Neuroscience Program \\ of the Georg-August University School of Science (GAUSS)
}

submitted by

Siv Vingill

from

Tynset, Norway

Göttingen 2016

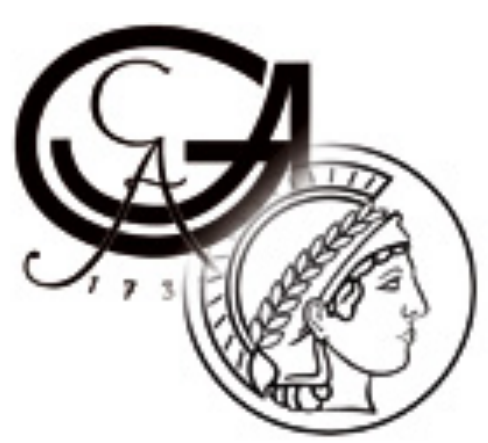




\section{Thesis Committee}

Dr. Judith Stegmüller, Department of Cellular and Molecular Neurobiology, Max-Planck Institute of Experimental Medicine

Prof. Dr. Thomas Bayer, Division of Molecular Psychiatry, University Medical Center Göttingen

Prof. Dr. Tiago Fleming Outeiro, Department of Neuro Degeneration and Restaurative Research, University Medical Center Göttingen

\section{Members of the Examination Board}

Referee: Dr. Judith Stegmüller, Department of Cellular and Molecular Neurobiology, MaxPlanck Institute of Experimental Medicine

$2^{\text {nd }}$ Referee: Prof. Dr. Thomas Bayer, Division of Molecular Psychiatry, University Medical Center Göttingen

\section{Further members of the Examination Board}

Prof. Dr. Tiago Fleming Outeiro, Department of Neuro Degeneration and Restaurative Research, University Medical Center Göttingen

Prof. Dr. Thomas Dresbach, Department of Anatomy and Embryology, University Medical Center Göttingen

Prof. Dr. Nils Brose, Department of Molecular Neurobiology, Max-Planck Institute of Experimental Medicine

Prof. Dr. Ralf Heinrich, Department of Cellular Neurobiology, Schwann-Schleiden Research Centre

Date of oral examination: 11.05.16 


\section{Affidavit}

I hereby declare that this $\mathrm{PhD}$ thesis entitled "Characterization of FBXO7

(PARK15) knockout mice modeling Parkinsonian-Pyramidal Syndrome" has been written independently with no other sources that quoted.

Siv Vingill

March, 2016

Göttingen, Germany 


\section{Contents}

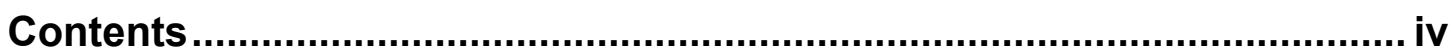

List of abbreviations ................................................................................. viii

List of figures ......................................................................................... xiv

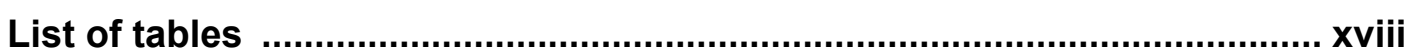

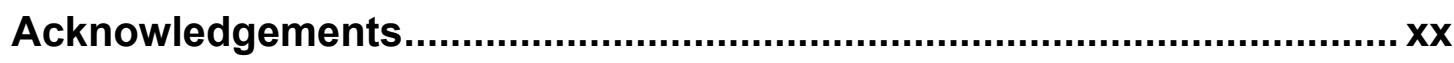

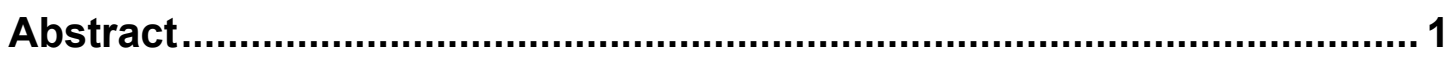

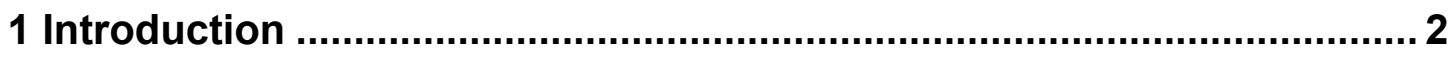

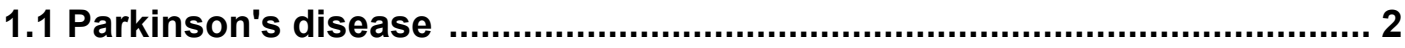

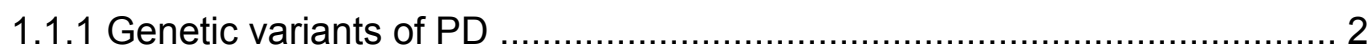

1.2 Rodent models of Parkinson's disease ..................................................... 4

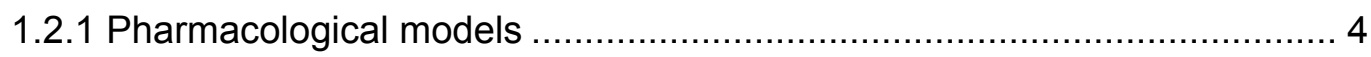

1.2.2 Knock-in mouse models ..................................................................... 5

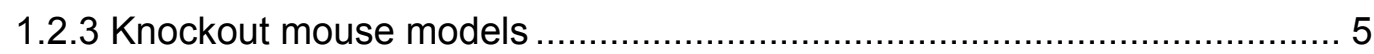

1.2.4 Novel animal models of PD ........................................................... 6

1.3 Molecular mechanisms of Parkinson's disease ....................................... 6

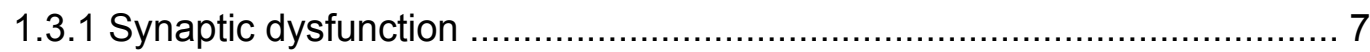

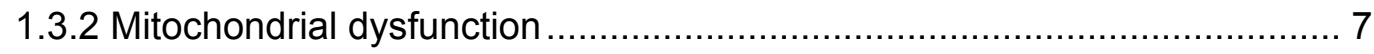

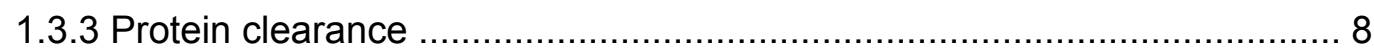

1.4 FBXO7

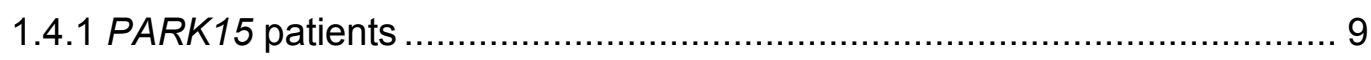

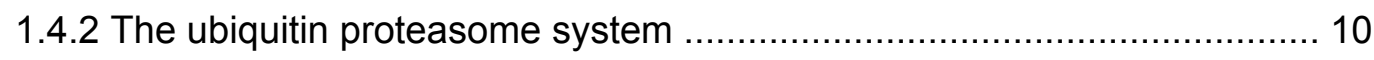

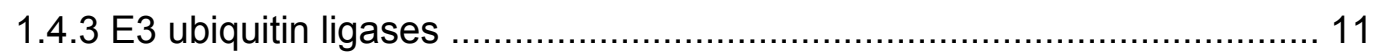

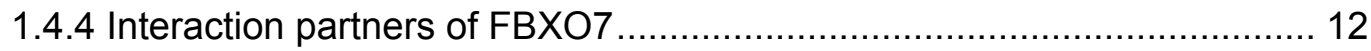

1.4.5 Proteasomal inhibitor 31 (PI31) ........................................................ 16

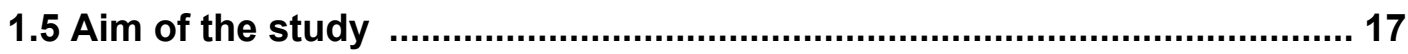

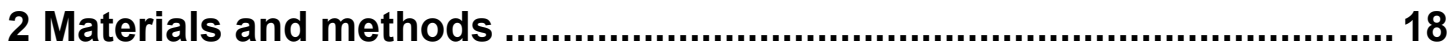

2.1 Reagents and equipment ……............................................................ 18

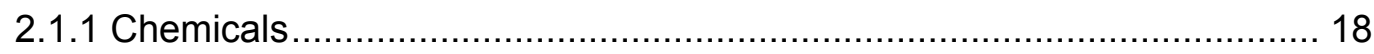

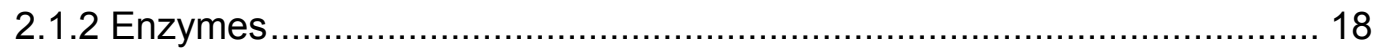

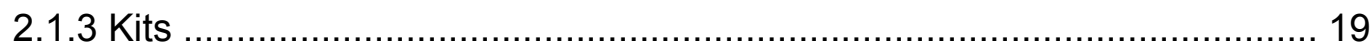




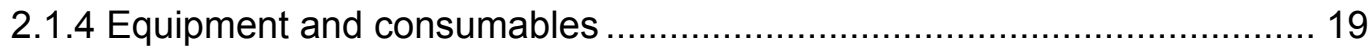

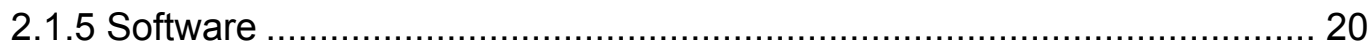

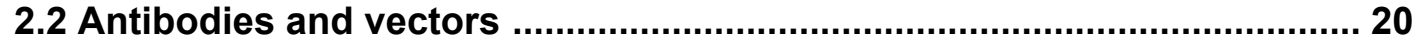

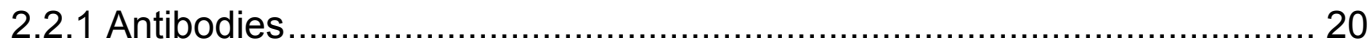

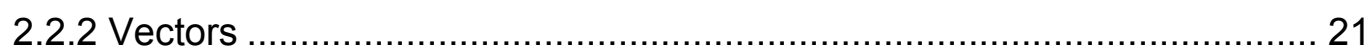

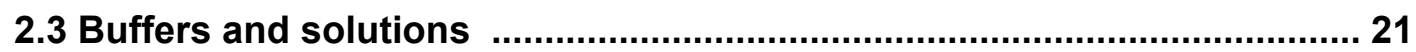

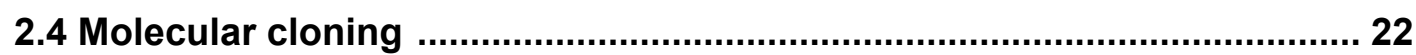

2.4.1 Generation of short hairpin RNA ..................................................... 22

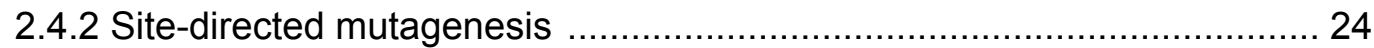

2.4.3 Generation of FBXO7 FP-domain deletion construct ............................. 25

2.4.4 Bacterial transformation and selection of positive clones....................... 26

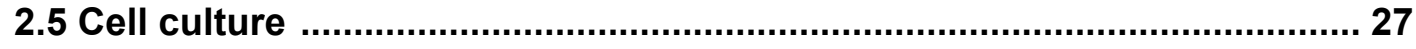

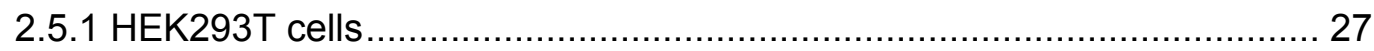

2.5.2 Cerebellar granule neuron culture .................................................. 27

2.5.3 Plasmid transfection of HEK293T cells ............................................... 27

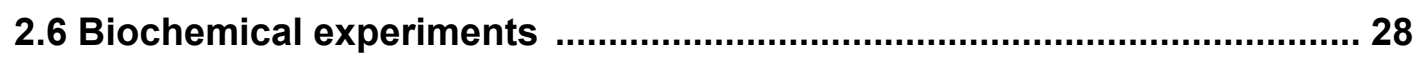

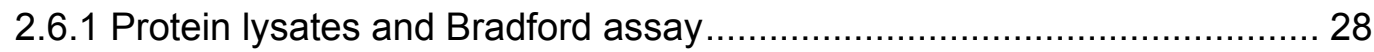

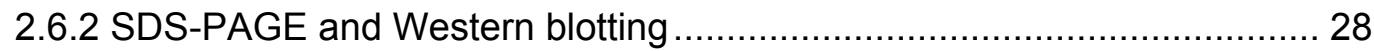

2.6.3 Co-immunoprecipitation (Co-IP) analysis ........................................... 29

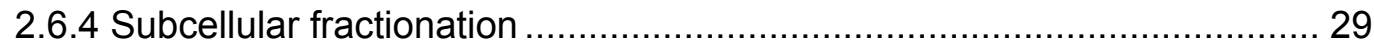

2.6.5 Purification of GST fusion proteins ....................................................... 29

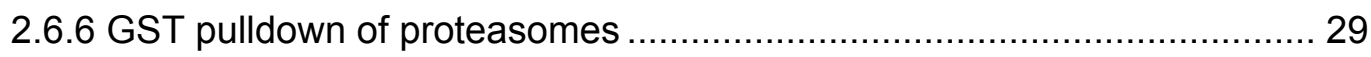

2.6 .7 Proteasomal activity assay ...................................................... 30

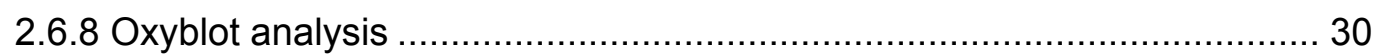

2.6.9 High Performance Liquid Chromatography (HPLC) ............................. 30

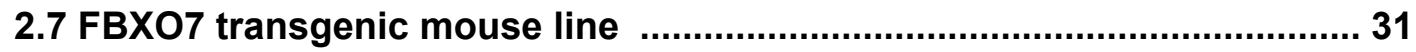

2.7.1 Generation of FBXO7 transgenic mouse line ..................................... 31

2.7.2 Isolation of genomic DNA and genotyping …..................................... 31

2.7.3 RNA isolation, cDNA synthesis and quantitative PCR ......................... 32

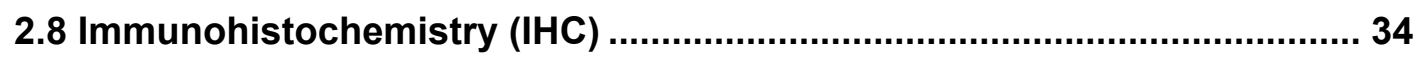

2.8.1 Transcardial perfusion and cryo-sectioning....................................... 34

2.8.2 Fluorescent IHC .................................................................... 34

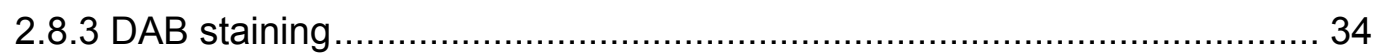

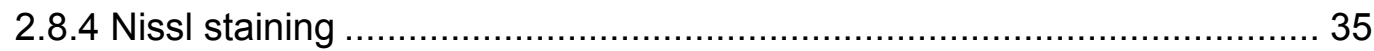

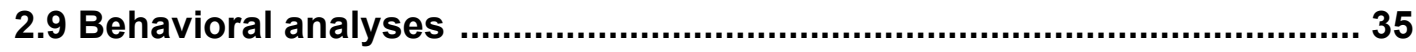

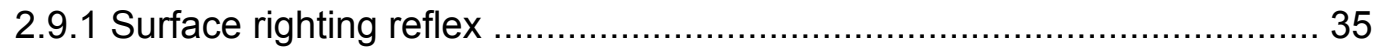

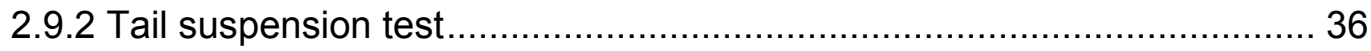




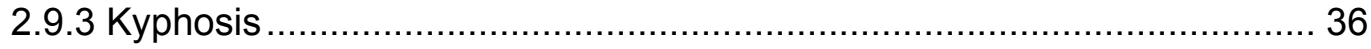

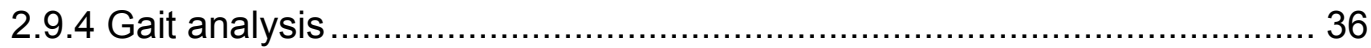

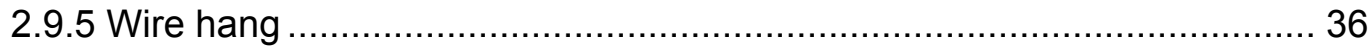

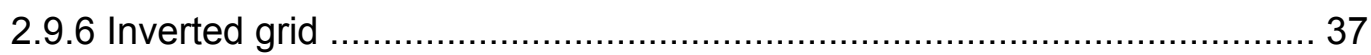

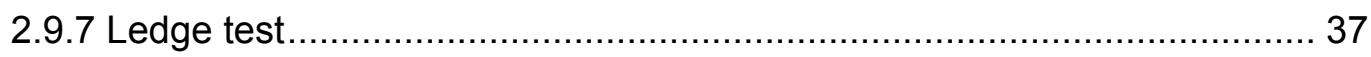

2.9.8 Balance beam test................................................................. 37

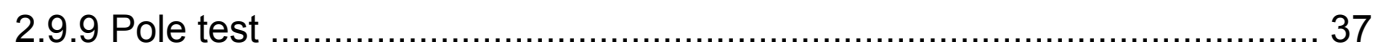

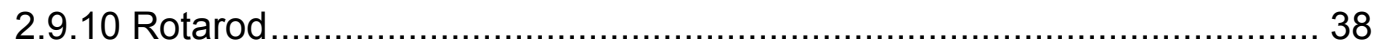

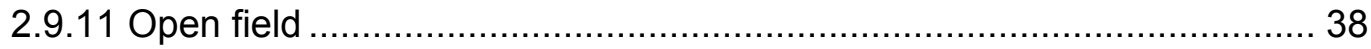

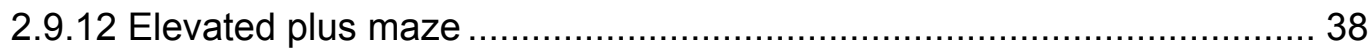

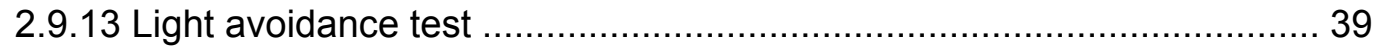

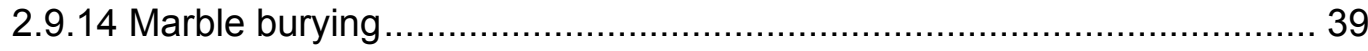

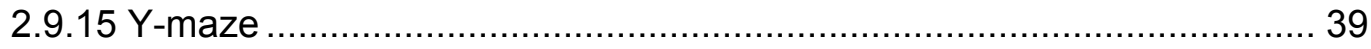

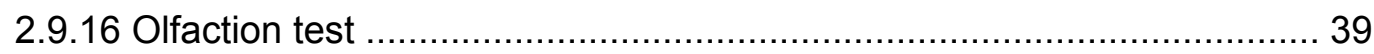

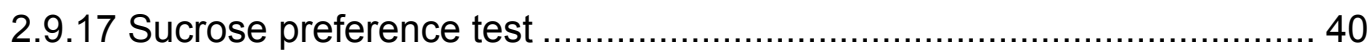

2.9.18 Laboratory animal behavior, observation, registration and

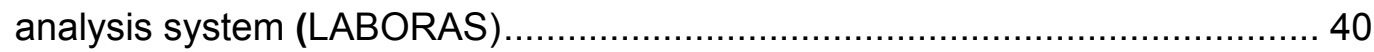

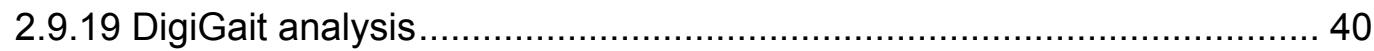

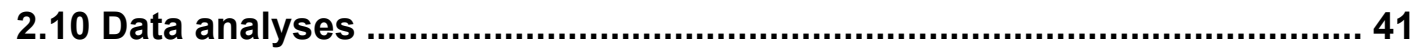

2.10.1 Western blot quantification .................................................... 41

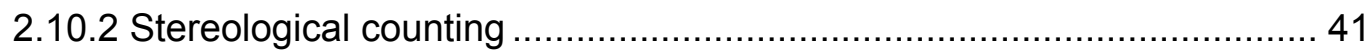

2.10.3 Quantification of IHC images....................................................... 41

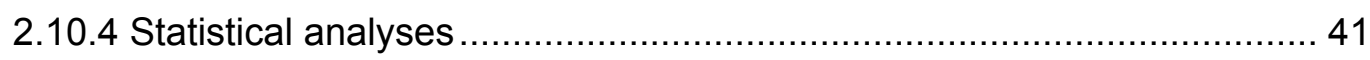

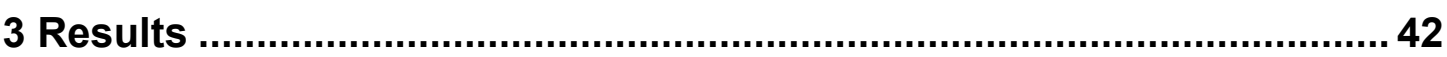

3.1 FBXO7 is ubiquitously expressed in rodents and present in the cytoplasm of cerebellar granule neurons ................................................... 42

3.1.1 Expression pattern of FBXO7 .................................................... 42

3.1.2 FBXO7 is expressed mainly in the cytoplasm in rat cerebellar

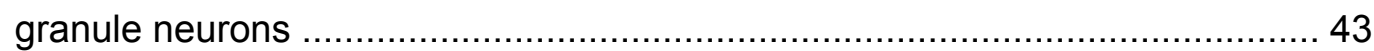

3.2 FBX07 positively regulates proteasome activity .................................... 44

3.3 Characterization of the novel FBXO7 knockout mouse model ................ 49

3.3.1 Generation of FBXO7 conventional knockout mice............................. 49

3.3.2 FBXO7-/- mice show retarded growth and premature death ................... 50

3.3.3 FBXO7-/- mice show diminished motor control at P18 .......................... 52

3.3.4 Heterozygous FBXO7 mice show normal behavior at 6 and 12

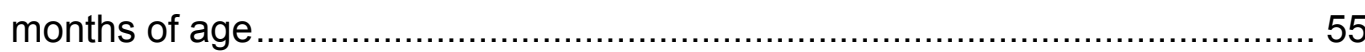


3.3.5 FBXO7-/- mice show normal dopaminergic neuron morphology and function

3.3.6 FBXO7-/- mice show reduced level of PI31, but no change in

proteasome activity or oxidation levels

3.4 NEX-Cre;fl/fl mice show pyramidal symptoms with stereotypic movements and hyperactivity 62

3.4.1 FBXO7 $\mathrm{fl} / \mathrm{fl}$ mice show normal behavior up to 12 months of age 62

3.4.2 FBXO7 protein expression is reduced in cortex of NEX-Cre;fl/fl mice

3.4.3 NEX-Cre;fl/fl mice show spasticity and decreased coordination 65

3.4.4 NEX-Cre;fl/fl mice show hyperactivity and stereotypic behavior

3.4.5 NEX-Cre;fl/fl mice show altered protein expression in the striatum.

3.5 TH-Cre;fl/fl mice show progressive motor decline and loss of dopamine in the striatum

3.5.1 TH-Cre;fl/fl mice show abnormal weight-regulation and hind limb clasping

3.5.2 TH-Cre;fl/fl mice show normal olfaction and anxiety phenotype 81

3.5.3 TH-Cre;fl/fl mice display a progressive loss of motor function 83

3.5.4 $\mathrm{TH}-\mathrm{Cre} ; \mathrm{fl} / \mathrm{fl}$ mice have reduced amount of dopamine in the striatum. 86

3.5.5 TH-Cre;fl/fl mice show astrogliosis in the midbrain 89

4 Discussion 91

4.1 FBXO7 as a proteasomal regulator 92

4.2 Systemic loss of FBXO7 is detrimental to the organism 93

4.3 Modeling the pyramidal symptoms seen in PARK15 patients 95

4.4 Loss of FBXO7 in catecholaminergic neurons 97

4.5 Loss of FBXO7 causes neuronal dysfunction 101

5 Conclusions and perspectives

Bibliography 104

Appendix. 120

Curriculum Vitae. 


\section{List of abbreviations}

${ }^{\circ} \mathrm{C}$

Degrees Celsius

6-OHDA 6-hydroxy dopamine

A Adenine

A/Ala Alanine

AAA-ATPase ATPases associated with diverse cellular activities

AD

Autosomal dominant

ADP

Adenosine diphosphate

ANOVA Analysis of variance

APC Anaphase-promoting complex

APP Amyloid precursor protein

APS Ammonium persulfate

AR Autosomal recessive

AraC cytosine-b-D-arabinofuranoside

ATP Adenosine triphosphate

ATP13A2 ATPase type 13A2

ATPase Adenylpyrophosphatase

$\beta$-Gal $\quad \beta$-Galactosidase

bp Basepairs

BME Basal Medium Eagle

BSA Bovine serum albumin

C Cytosine

CB Cerebellum

Cdk6 Cell division protein kinase 6

cDNA complementary DNA

clAP1 Cellular inhibitor of apoptosis 1

CIT-SPECT 2-beta-carboxymethoxy-3-beta-(4-iodophenyl) tropane -SPECT

cm Centimeter

Co-IP Co-Immunoprecipitation

CP Core particle

Cre Cyclization recombinase

Crm1 Chromosomal maintenance 1

CTX Cortex 


\begin{tabular}{|c|c|}
\hline DAB & 3-3'-diaminobenzidine \\
\hline DAPI & 4'6-diamidino-2-phenylindole \\
\hline DAT & Dopamine transporter \\
\hline DIV & Days in vitro \\
\hline DMEM & Dulbecco's modified Eagle's medium \\
\hline DmPI31 & Drosophila melanogaster proteasome inhibitor 31 \\
\hline DNA & Deoxyribonucleic acid \\
\hline DNAJC6 & DnaJ (Hsp40) homolog, subfamily C, member 6 \\
\hline DNP & dinitrophenyl \\
\hline DNPH & 2,4-dinitrophenylhydrazine \\
\hline dNTP & Deoxyribonucleotide triphosphate \\
\hline DOPAC & 3,4-dihydroxyphenylacetic acid \\
\hline DTT & Dithiothreitol \\
\hline E/Glu & Glutamic acid \\
\hline E1 & Ubiquitin-activating enzyme \\
\hline E2 & Ubiquitin-conjugating enzyme \\
\hline E3 & Ubiquitin ligase enzyme \\
\hline E18 & Embryonic day 18 \\
\hline ECL & Enhanced chemiluminescence \\
\hline EDTA & Ethylenediaminetetraacetic acid \\
\hline EGTA & Ethylene glycol tetraacetic acid \\
\hline EIF4G1 & Eukaryotic translation initiation factor 4 gamma, 1 \\
\hline En2 SA & Mouse engrailed 2 gene splice acceptor \\
\hline EOPD & Early-onset PD \\
\hline ES & Embryonic stem cells \\
\hline FBP & F-box protein \\
\hline FBXL & F-box protein with leucine-rich repeats \\
\hline FBXO & F-box protein with other domain motifs \\
\hline FBXW & F-box protein with WD40 domains \\
\hline FBS & Fetal bovine serum \\
\hline FLP & Flippase \\
\hline FP & FBOX7-PI31 interaction domain \\
\hline FRT & FLP-recognition target \\
\hline$g$ & Gravity \\
\hline g & Gram \\
\hline G & Guanine \\
\hline G/Gly & Glycine \\
\hline
\end{tabular}




\begin{tabular}{|c|c|}
\hline GAD & Gracile axonal dystrophy \\
\hline GFAP & Glial fibrillary acidic protein \\
\hline GFP & Green fluorescent protein \\
\hline GIGYF2 & GRB10 interacting GYF protein 2 \\
\hline GRB10 & Growth factor receptor bound protein 10 \\
\hline GST & Glutathione S-transferase \\
\hline gt & Goat \\
\hline GYF & Glycine-tyrosine-phenylalanine domain \\
\hline HBSS & Hank's Balanced Salt Soulution \\
\hline HCP & Hippocampus \\
\hline HECT & Homologous to E6-associated protein carboxy terminal \\
\hline HEK293T & Human Embryonic Kidney 293T cells \\
\hline HEPES & 4-(2-hydroxyethyl)-1-piperazineethanesulfonic acid \\
\hline HPLC & High Performance Liquid Chromatography \\
\hline HRP & Horseradish peroxidase \\
\hline hs & Homo sapiens \\
\hline HS & Horse serum \\
\hline HtrA2 & HtrA serine peptidase 2 \\
\hline HURP & Hepatoma up-regulated protein \\
\hline HVA & Homovanillic acid \\
\hline $\mathrm{I} / \mathrm{lle}$ & Isoleucine \\
\hline IB & Immunoblot \\
\hline lba1 & Ionized calcium-binding adapter molecule 1 \\
\hline ICC & Immunocytochemistry \\
\hline IgGH & Immunoglobulin G heavy chain \\
\hline $\lg G L$ & Immunoglobulin G light chain \\
\hline IHC & Immunohistochemistry \\
\hline IL & Interleukin \\
\hline IP & Immunoprecipitation \\
\hline IPTG & Isopropyl $\beta$-D-1-thiohalactopyranoside \\
\hline IRES & Internal ribosome entry site \\
\hline K/Lys & Lysine \\
\hline Kan & Kanamycin \\
\hline kb & Kilobase \\
\hline KD & Knock-down \\
\hline kDa & Kilodalton \\
\hline kg & kilogram \\
\hline
\end{tabular}




KO Knockout

L Liter

LC Locus coeruleus

LC1 Light chain 1

L/Leu Leucine

LRRK2 Leucine-rich repeat kinase 2

m Mouse

M/Met Methionine

mA Milliampere

MAP1B Microtubule-associated protein 1B

mg Milligram

ml Milliliter

$\mathrm{mm} \quad$ Mus musculus

mRNA Messenger RNA

$\mu \mathrm{g} \quad$ Microgram

$\mu \mathbf{l} \quad$ Microliter

$\mu$ Micromolar

$\mu \mathrm{m} \quad$ Micrometer

mM Milimolar

mm milimeter

MPP+ 1-Methyl-4-phenyl-pyridin

MPTP 1-methyl-4-phenyl-1,2,3,6-tetrahydropyridine

ms millisecond

mV millivolt

NeuN Neuronal nuclei

NEX Neuronal helix-loop-helix protein-1

NF- $\mathbf{k} \quad$ Nuclear factor 'kappa-light-chain-enhancer' of activated B-cells

ng Nanogram

nm Nanometer

OCT Optimal cutting temperature

OD Optical density

OE Over-expression

P/Pro Proline

$\mathbf{P}(n) \quad$ Postnatal Day (n)

pA Polyadenylation

PARK PD-associated gene locus

PBS Phosphate-buffered saline 


$\begin{array}{ll}\text { PBST } & \text { Phosphate-buffered saline with Triton-X } \\ \text { PCR } & \text { Polymerase chain reaction } \\ \text { PD } & \text { Parkinson's disease } \\ \text { PFA } & \text { Paraformaldehyde } \\ \text { PFFs } & \text { Preformed fibrils } \\ \text { PLA2G6 } & \text { Phospholipase A2 group VI } \\ \text { PMSF } & \text { Phenylmethanesulfonylfluoride } \\ \text { PRR } & \text { Proline-rich region } \\ \text { PSG } & \text { Penicillin/Streptomycin with L-Glutamine } \\ \text { PI31 } & \text { Proteasomal inhibitor 31 } \\ \text { PSMA2 } & \text { Proteasomal subunit alpha 2 } \\ \text { PSMB5 } & \text { Proteasomal subunit beta 5 } \\ \text { PINK1 } & \text { PTEN Induced Putative Kinase 1 } \\ \text { pmol } & \text { picomolar } \\ \text { PTEN } & \text { Phosphatase and tensin homolog } \\ \text { qPCR } & \text { Quantitative PCR } \\ \text { RIArg } & \text { Arginine } \\ \text { Rad } & \text { Radiation sensitive } \\ \text { rb } & \text { Rabbit } \\ \text { Rbx1 } & \text { RING-box protein 1 } \\ \text { RING } & \text { Really Interesting New Gene } \\ \text { RIPA } & \text { Radioimmunoprecipitation assay buffer } \\ \text { RNA } & \text { Ribonucleic acid } \\ \text { RNAi } & \text { RNA interference } \\ \text { ROS } & \text { Reactive oxygen species } \\ \text { RP } & \text { Regulatory Particle } \\ \text { rpm } & \text { Revolutions per minute } \\ \text { Rpn } & \text { RP non-ATPase } \\ \text { Rpt } & \text { RP triphosphatase } \\ \text { RT } & \text { Room temperature } \\ \text { SES } & \text { Sedimentation region } \\ \text { Second } \\ \text { SEine }\end{array}$




\begin{tabular}{|c|c|}
\hline shRNA & Short hairpin RNA \\
\hline SKP1 & S-phase kinase-associated protein 1 \\
\hline SN & Substantia nigra \\
\hline SNCA & Synuclein, alpha (non A4 component of amyloid \\
\hline SNP & Single nucleotide polymorphism \\
\hline SP1 & Specificity protein 1 \\
\hline SPECT & Single-photon Emission Computed Tomography \\
\hline SYNJ1 & Synaptojanin 1 \\
\hline $\mathbf{T}$ & Thymine \\
\hline T/Thr & Threonine \\
\hline TEMED & Tetramethylethylenediamine \\
\hline TH & Tyrosine hydroxylase \\
\hline TNF & Tumor necrosis factor \\
\hline TRAF2 & TNF receptor-associated factor 2 \\
\hline TUNEL & TdT-mediated dUTP nick-end labeling \\
\hline Ub & Ubiquitin \\
\hline Ubl & Ubiquitin-like \\
\hline UCHL1 & Ubiquitin carboxyl-terminal esterase L1 \\
\hline UIM & Ubiquitin-interacting motif \\
\hline UPS & Ubiquitin proteasome system \\
\hline UV & Ultra violet \\
\hline $\mathbf{v}$ & Volts \\
\hline V/Val & Valine \\
\hline VGLUT & Vesicular glutamate transporter \\
\hline VPS35 & Vacuolar protein sorting-associated protein 35 \\
\hline W/Trp & Tryptophan \\
\hline WB & Western blot \\
\hline WT & Wild type \\
\hline $\mathbf{X}$ & Termination \\
\hline
\end{tabular}




\section{List of figures}

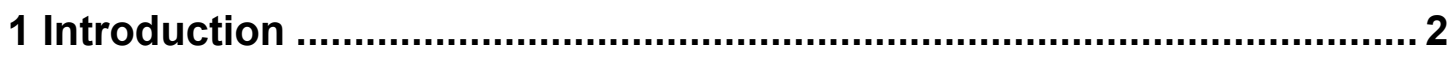

1.1 Schematic of the ubiquitin proteasome system ....................................... 11

1.2 Schematic representation of the Skp1-cullin1-F-box protein (SCF)

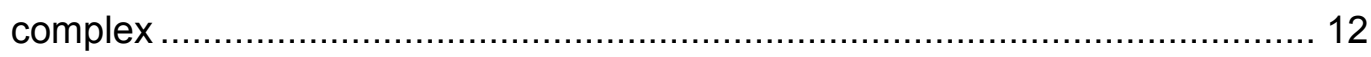

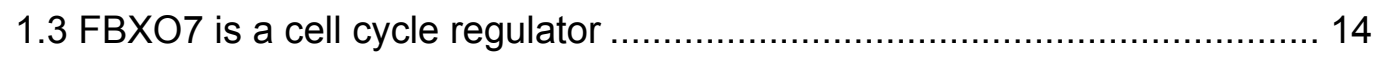

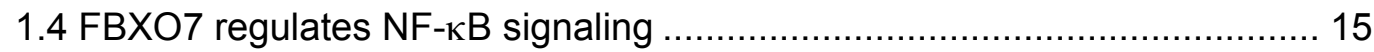

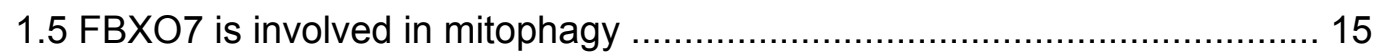

1.6 Domain structures of FBXO7 and PI31 ................................................ 16

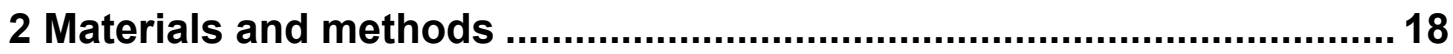

2.1 Schematic of open field for adult animals ................................................ 38

2.2 Schematic of olfaction test ................................................................. 40

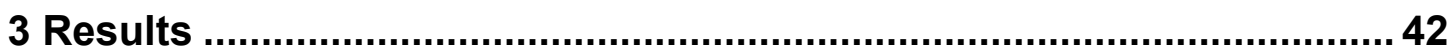

3.1.1 FBXO7 shows ubiquitous expression in rat tissue .............................. 42

3.1.2 FBXO7 is expressed in the brain throughout development..................... 43

3.1.3 FBXO7 is expressed mainly in the cytoplasm of cultured

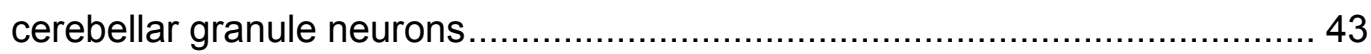

3.2.1 The FP domain of FBXO7 is required for interaction with $\mathrm{PI} 31 \ldots \ldots \ldots \ldots . . . . .44$

3.2.2 Mutant FBXO7 proteins interact with PI31 ......................................... 45

3.2.3 FBXO7 and PI31 interact with the proteasome ................................... 45

3.2.4 Validation of shRNA constructs for FBXO7, PSMA2 and PI31 ............... 46

3.2.5 PI31 interacts with PSMA2 through FBXO7 and may affect its

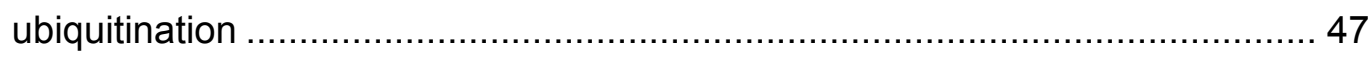

3.2.6 Knockdown of PSMA2 and FBXO7, but not PI31, lowers

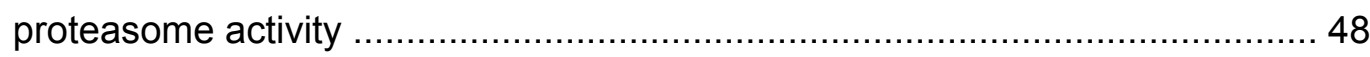

3.2.7 Overexpression of FBXO7 and PI31 do not influence

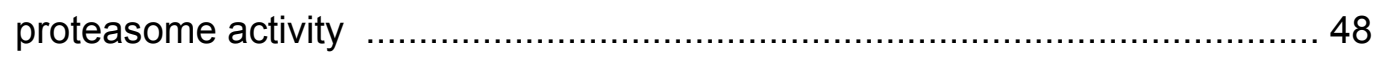

3.3.1 Targeting cassette for homologous recombination of $F B X O 7 \ldots \ldots \ldots \ldots \ldots . . . . . .49$

3.3.2 Disruption FBXO7 gene, mRNA and protein expression in FBXO7-/- mice. 
3.3.3 FBXO7-/- mice show premature lethality. 51

3.3.4 FBXO7 knockout mice show retarded growth ..................................... 52

3.3.5 FBXO7-/- mice show normal surface righting reflex at P5 .................... 52

3.3.6 FBXO7-/- mice display mild kyphosis and hind limb clasping ................. 53

3.3.7 FBXO7-/- mice display normal ambulation and slight gait

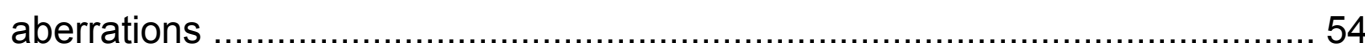

3.3.8 FBXO7-/- mice show diminished motor control ..................................... 54

3.3.9 FBXO7+/- mice show normal body characteristics and muscle

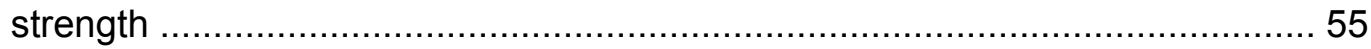

3.3.10 FBXO7+/- mice show normal ambulation........................................ 56

3.3.11 FBXO7+/- mice show normal anxiety levels .................................... 56

3.3.12 FBXO7+/- mice show normal motor function....................................... 57

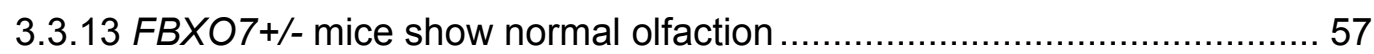

3.3.14 FBXO7-/- mice show no substantial loss of dopaminergic neurons at $\mathrm{P} 18$ 58

3.3.15 FBXO7-/- animals show no evident loss of transporters in the striatum. 59

3.3.16 FBXO7-/- mice show no evident loss of dopamine in the striatum. 60

3.3.17 FBXO7-/- mice show a decrease in PI31 levels . 61

3.3.18 Cultured cerebellar granule neurons of FBXO7-/- mice show no decrease in proteasome activity

3.3.19 FBXO7-/- mice show no difference in oxidized protein levels in the cortex.

3.4.1 FBXO7 fl/fl show no abnormal behavioral phenotype 63

3.4.2 FBXO7 protein and mRNA expression is reduced in cortex of NEX-Cre;fl/fl mice.

3.4.3 NEX-Cre;fl/fl show no increase in weight and a strong hind limb clasping up to 4 months of age

3.4.4 NEX-Cre;fl/fl mice show progressive decrease in motor coordination 67

3.4.5 NEX-Cre;fl/fl mice show severe hyperactivity...................................... 68

3.4.6 NEX-Cre;fl/fl mice show stereotypic circling ....................................... 69

3.4.7 NEX-Cre;fl/fl mice show increased rearing and scratching, but no difference in grooming and climbing............................................................ 70

3.4.8 NEX-Cre;fl/fl mice show decreased marble moving and burying ............ 71 
3.4.9 NEX-Cre;fl/fl mice show no aberrations in spatial memory, but patterned

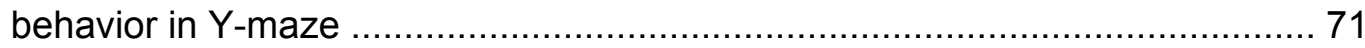

3.4.10 NEX-Cre;fl/fl mice show stereotypic movement pattern ....................... 72

3.4.11 NEX-Cre;fl/fl mice show increased eating and reduced drinking frequency

3.4.12 NEX-Cre;fl/fl mice show decreased anxiety in the elevated plus maze test

3.4.13 NEX-Cre;fl/fl mice show reduced change of location in light/ dark paradigm 74

3.4.14 NEX-Cre;fl/fl mice show normal olfaction 74

3.4.15 Cortical thickness and number of neurons in layer $V$ seems normal in NEX-Cre;fl/fl mice

3.4.16 NEX-Cre;fl/fl animals show a decrease in VGLUT1 expression in the striatum

3.4.17 NEX-Cre;fl/fl mice show an increase in DAT expression in the striatum.

3.4.18 NEX-Cre;fl/fl mice show an increase of dopamine in the striatum 77

3.4.19 NEX-Cre;fl/fl mice show a decrease in PI31 levels 78

3.4.20 NEX-Cre;fl/fl mice show no difference in oxidized protein levels in cortex.

3.5.1 Verification of $\mathrm{TH}$-cre; $\mathrm{fl} / \mathrm{fl}$ mouse line. 79

3.5.2 TH-Cre;fl/fl mice show increased weight at 6 months and are either over- or underweight at 12 months of age 80

3.5.3 TH-Cre;fl/fl mice are not anhedonic at 2 months of age ....................... 81

3.5.4 TH-Cre;fl/fl mice show hind limb clasping at 12 months of age ............... 81

3.5.5 TH-Cre;fl/fl mice show no anxiety phenotype ....................................... 82

3.5.6 TH-Cre;fl/fl mice show no loss of olfaction ........................................... 82

3.5.7 TH-Cre;fl/fl mice move less at 12 months of age ................................. 83

3.5.8 TH-Cre;fl/fl mice show progressively slower movements ....................... 83

3.5.9 TH-Cre;fl/fl mice show progressive loss of motor function ...................... 84

3.5.10 TH-Cre;fl/fl mice show progressive loss of fine motor control ................ 84

3.5.11 TH-Cre;fl/fl mice show no loss of neurons in substantia nigra .............. 86

3.5.12 TH-Cre;fl/fl mice show little or no striatal terminal loss ........................ 87

3.5.13 TH-Cre;fl/fl mice show normal expression of TH and DAT protein levels in the striatum at 2 months of age 
3.5.14 TH-Cre;fl/fl mice show downregulation of DAT protein levels in the striatum at 12 months of age

3.5.15 TH-Cre;fl/fl mice show downregulation level of dopamine levels in the striatum

3.5.16 TH-Cre;fl/fl animals show increased GFAP positive area in the midbrain 


\section{List of tables}

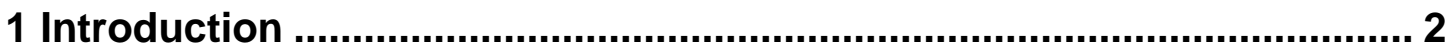

1.1 Loci and genes associated with Parkinson's disease .................................

2 Materials and methods......................................................................... 18

2.1.1 Enzymes used in this study ......................................................... 18

2.1.2 Commercial kits used in this study .............................................. 19

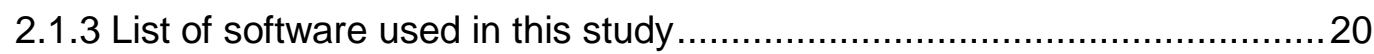

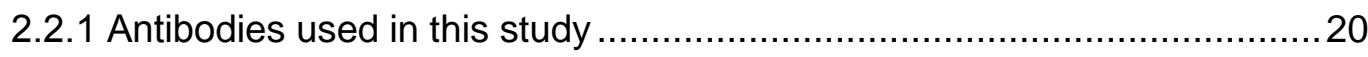

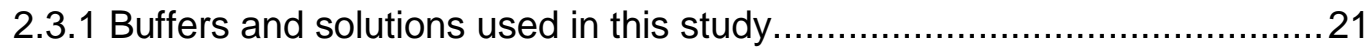

2.4.1 Target sequences for shRNA .................................................... 23

2.4.2 PCR reaction for generation of shRNA ......................................... 23

2.4.3 PCR reaction for generation of point mutations .................................... 24

2.4.4 PCR reaction for site-directed mutagenesis.........................................2 24

2.4.5 PCR program for site-directed mutagenesis ......................................... 24

2.4.6 PCR reaction for fragment amplification .......................................... 25

2.4.7 PCR program for fragment amplification............................................ 25

2.4.8 PCR reaction for fragment fusion ................................................. 26

2.4.9 Ligation of fragment into vector ................................................. 26

2.6.1 Composition of Tris-SDS polyacrylamide gels.....................................28

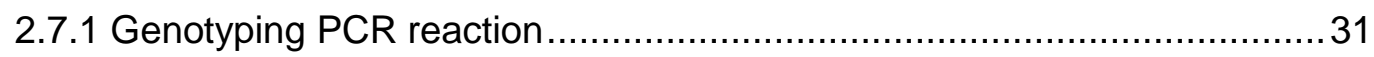

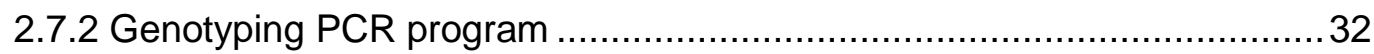

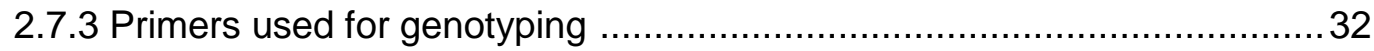

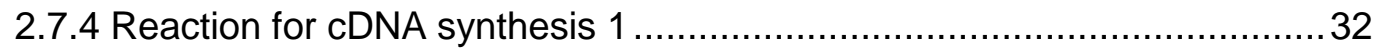

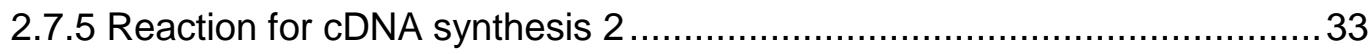

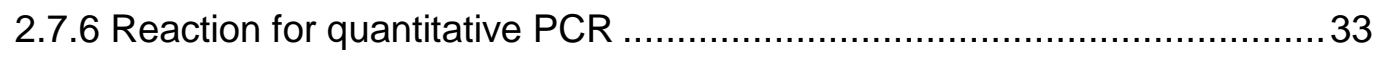

2.7.7 Primers for quantitative PCR used in this study ................................. 33

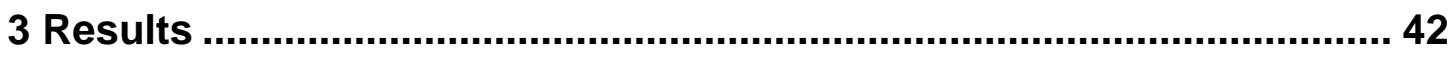

3.3.1 Ratio of mice pups born to FBXO7+/- parents ....................................51 
3.4.1 Loss of FBXO7 in NEX-Cre;fl/fl mice is not embryonically lethal 64

3.4.2 NEX-Cre; $\mathrm{fl} / \mathrm{fl}$ mice stereotypic circling is uni-directional within test session. 68

3.5.1 Reason for sacrifice of TH-Cre mice 80

3.5.2 TH-Cre;fl/fl mice show alteration in gait parameters at 8 months of age 85

Appendix 120

A1 Mutations and clinical features described in PARK15 patients 120 
"The grass was greener. The light was brighter.

The taste was sweeter. The nights of wonder. With friends surrounded."

Pink Floyd, "High Hopes"

\section{Acknowledgements}

I could not have finished my PhD without the support from a lot of people. Firstly, I want to thank Judith for your great supervision. I appreciate your patience, your support and the freedom you allowed me during my $\mathrm{PhD}$. Through this project I have become a scientist and that stems from the discussions we have had and the guidance you have given me. Secondly, I thank my thesis committee, Prof. Dr. Thomas Bayer and Prof. Dr. Tiago Outeiro, for your valuable input during fruitful thesis committee discussions.

The IMPRS program has some people who have gone above and beyond to make our PhD journeys smooth sailing. Michael, Sandra and Mirja, thank you so much for the support, the discussions and the inspiring retreats. Steffen, Kerstin, Kirsten, Antje, Katrin, Mandy and Susanne, thank you so much for everything you do for the PhD community and for the excellent cooperation we have had through my time as a part of the GGNB.

I also want to thank my collaboration partners for their incredible help and support. Camille Lancelin, for the great work with the DigiGait, Dr. Lars Tatenhorst for excellent HPLC analysis and Prof. Dr. Ekrem Dere for all the good advice and relentless experimental support.

None of this would be possible without AG Awesome. Nicola, I wouldn't have gotten a single experiment done in the beginning without your steel control. Mayur, you are my mentor and councilor, and I owe you a great deal of coffee and advice by now (and some magnificent pranks). Shih-Ju, you make the ups seem more up and the downs seem less down (in my mind this is accompanied by ukulele). Without you the last years would have been way less interesting. Anna, Alina, Annika, Guergana and Madhu you made the coziest (yes, it's a word) lab atmosphere one could wish for. David, thank you for being a great project partner and for all our interesting discussions. Chaitali and Sabitha, you guys made the summer of 2015 memorable, let's all meet for coffee and chocolate soon! And to David, Shih-Ju, Mayur and Ludo, thank you for the thorough input you provided on my thesis and the supply of food and coffee you more or less volunteered.

I could never have done anything in life if it wasn't for the support of my family. Dear mamma and pappa, you are my advisors and my mental strength. The biggest reason I'm crazy for Christmas is that I always go home. Wherever I am in the world, I know I have 
one true home. Eystein Magnus, you're the best brother a sister can wish for. I hope you know how much you mean to me. Bodil, we are sometimes so alike, and sometimes so different. Maybe you're the one I have been the angriest at, but also one of the most loved ones. Thank you for calling me out when I am wrong and still always forgiving me.

On the same note I thank Tuva and Synne. You are sisters in everything but name, and I really don't know what I would have done without you. You are my psychologists, my partners in crime, my inspirations and my best friends. And to Kjell, Gjermund, Amund, Bjørnar, Tore and the rest of Vingelen/Tolga, a big hug! "Ei kjem tu Vinnila og ei gjer ke ei vil!" Martin, An, Kristo and Eivind, thank you so much for the football, the board games, the Sunday dinners and for making the Bachelor studies such a lot of fun!

Last, but definitely not least, to my AKB family. Vingelen is my true home, but you guys have made my time in Göttingen some of the best years of my life. I know I will always find room where you are, and you will always find a home with me. Going through this experience together, you have shaped my way of thinking and showed me such a lot of joy in life! Marta, thank you for keeping us sane and telling us everything will be fine. Paola, thank you for the music, the songs you're singing, thanks for all the joy you're bringing. Victor, you're a firework, thank you for inspiring me to be a better person, always! Irena, you wonderful roomie, thanks for all the coffee, food, patience and emotional support! Mateusz, thank you for so much, but especially for enriching my vocabulary in the weirdest ways and keeping me real at all times! And lastly, Goran: thank you for always being there when support is needed! 


\section{Abstract}

The second most common neurodegenerative disease, Parkinson's disease (PD), is caused by loss of dopaminergic neurons in the substantia nigra leading to impaired motor control. Genetic analyses have revealed recessively and dominantly inherited mutations in the so-called PARK genes that cause symptoms reminiscent of those of PD. Mutations in the FBXO7 gene (PARK15) have been linked to several families presenting with Parkinsonian-Pyramidal Syndrome, but its precise role in neurons remains unknown. Proteasomal dysfunction has previously been linked to PD. Owing to the previously published finding that $\mathrm{FBXO} 7$ interacts with the proteasomal modulator $\mathrm{PI} 31$, I investigated the role of $\mathrm{FBXO} 7$ in proteasomal regulation and found in loss-of-function experiments that FBXO7 promotes proteasome activity. To model the PARK15 syndrome in mice, we generated a conventional FBXO7 knockout mouse line. With behavioral experiments, I showed that these mice present early onset motor defects and premature death. As FBXO7 is ubiquitously expressed in the brain, we dissected the pyramidal and Parkinsonian phenotype using a conditional knockout strategy. I found that loss of FBXO7 in pyramidal forebrain neurons caused severe motor deficits reminiscent of the pyramidal symptoms seen in PARK15 patients, in addition to stereotypic behavior. I could show that the glutamatergic transporter level was downregulated in the striatum of these mice, along with an increase in dopamine. On the other hand, loss of $\mathrm{FBXO} 7$ in murine catecholaminergic neurons lead to a progressive loss of fine motor control with reduced ambulation in the end stage, which can be interpreted as a Parkinsonian phenotype. This was accompanied by a significant decrease of striatal dopamine, without neuronal loss. Taken together, this study shows FBXO7 as a regulator of the $26 \mathrm{~S}$ proteasome and characterizes novel mouse models for investigation of Parkinsonian-Pyramidal Syndrome. 


\section{Introduction}

\subsection{Parkinson's disease}

With an aging population the world faces new challenges in the shape of age-related diseases. Second only to Alzheimer's disease, Parkinson's disease (PD) affects about 6 million people worldwide and is one of the most common neurodegenerative disorders known today (Dorsey et al., 2007). It is a complex motor disease whose symptoms comprise bradykinesia, resting tremor, rigidity and postural instability. However, additional symptoms such as cognitive decline, mood disorders and sleep disturbances are mostly present and further contribute to the discomfort of patients' every day life. The underlying reason for PD symptoms is a loss of dopaminergic neurons in the substantia nigra, but what causes these neurons to die is still debated. PD research aims at easing the pain and giving hope to patients, while simultaneously saving the society costly symptomatic treatment. This quest begins with identifying the pathogenic mechanisms causing the disease and requires a translational approach involving meaningful animal models.

\subsubsection{Genetic variants of PD}

Most cases of Parkinson's disease are of idiopathic origin with only about $10 \%$ of patients reporting a family history of PD (Thomas and Beal, 2007). However, in recent years, several families have been diagnosed with familiar PD caused by mutations in identified genetic loci, the so-called PARK loci (Table 1.1). Although these genetic variants are rare, they can offer valuable insight into the signaling pathways that are rendered dysfunctional and hence shed light on therapeutic strategies for both familiar and sporadic forms of PD.

The first gene to be associated with PD was SNCA (Polymeropoulos et al., 1997), which encodes for the protein $\alpha$-synuclein. This protein was later shown to be a major constituent of the so-called Lewy bodies; intra-neuronal protein deposits in the brain known as the pathological hallmark of PD (Spillantini et al., 1997). Patients have been found to have point-mutations or duplication events in the SCNA gene (PARK1/4) (Nuytemans et al., 2010). These mutations are inherited in an autosomal dominant manner and the severity of the symptoms correlate with increased gene number, suggesting a gain-of-function toxicity of the dysfunctional protein. The symptoms in PARK1/4 patients are often early-onset (>55 years), with dementia as a secondary 
symptom. Another PD variant that is inherited in the same manner is caused by point mutations in the PARK8 gene encoding leucine-rich-repeat kinase 2 (LRRK2) (Brice, 2005; Lesage et al., 2005; Ozelius et al., 2006). This is the most prevalent PD gene known so far and is found both in late-onset familiar and sporadic PD, with the familiar cases closely resembling the symptoms of the sporadic variant. LRRK2 is a protein kinase whose mutant variants are also thought to confer a toxic gain-of-function.

Three other genes that have been unequivocally associated with PD are PARK2, PARK6 and PARK7, which encode the E3 ubiquitin ligase parkin, PTEN-induced putative kinase 1 (PINK1), and the antioxidant stress sensor DJ-1, respectively (Bonifati et al., 2003; Kitada et al., 1998; Valente et al., 2004). These syndromes are inherited in an autosomal recessive manner and the symptoms seen in these patients are often early-onset and caused by point mutations in their respective genes. PARK2, 6 and 7 are symptomatically indistinguishable and cause early-onset PD. Mutations in parkin are found in $77 \%$ of all juvenile Parkinson's cases (Lucking et al., 2000). All these genes are thought to be rendered dysfunctional by their mutations, suggesting that alterations in protein function cause PD symptoms in these patients.

Additional genes and loci are associated with PD, both as isolated cases and risk factors, but many of these remain controversial or unconfirmed (Klein and Westenberger, 2012). So far 20 loci have been included in the PARK gene list. Some genes are associated with syndromes that are not strictly Parkinsonian, others are either uncharacterized genes or genetic variants, which are predominantly linked with other syndromes (Table 1.1).

Table 1.1 Loci and genes associated with Parkinson's disease. Modified from Klein and Valadas (Klein and Westenberger, 2012; Valadas et al., 2015). AD=Autosomal dominant, $A R=$ Autosomal recessive, $P D=P a r k i n s o n ' s$ disease, $E O P D=E a r l y-o n s e t P D$.

\begin{tabular}{|c|c|c|c|c|c|}
\hline $\begin{array}{l}\text { PARK } \\
\text { locus }\end{array}$ & $\begin{array}{l}\text { Clinical } \\
\text { phenotype }\end{array}$ & Inheritance & Gene & Pathology & Status \\
\hline PARK1/4 & EOPD & $\mathrm{AD}$ & SNCA & Lewy bodies & Confirmed \\
\hline PARK2 & EOPD & AR & parkin & Lewy bodies & Confirmed \\
\hline PARK3 & Classical PD & $A D$ & unknown & Lewy bodies & Unconfirmed \\
\hline PARK5 & Classical PD & $A D$ & UCHL1 & Unknown & Unconfirmed \\
\hline PARK6 & EOPD & AR & PINK1 & $\begin{array}{l}\text { Lewy bodies ( } 1 \\
\text { case) }\end{array}$ & Confirmed \\
\hline PARK7 & EOPD & AR & $D J-1$ & Unknown & Confirmed \\
\hline PARK8 & Classical PD & $\begin{array}{l}A D \text { and risk } \\
\text { factor }\end{array}$ & LRRK2 & $\begin{array}{l}\text { Lewy bodies } \\
\text { (usually) }\end{array}$ & Confirmed \\
\hline PARK9 & $\begin{array}{l}\text { Kufor-Rakeb } \\
\text { syndrome }\end{array}$ & AR & ATP13A2 & Unknown & Confirmed \\
\hline PARK10 & Classical PD & Risk factor & unknown & Unknown & $\begin{array}{l}\text { Confirmed } \\
\text { susceptibility locus }\end{array}$ \\
\hline PARK11 & Late-onset PD & $\begin{array}{l}A D \text { or risk } \\
\text { factor }\end{array}$ & GIGYF2 & Unknown & $\begin{array}{l}\text { Not independently } \\
\text { confirmed }\end{array}$ \\
\hline
\end{tabular}




\begin{tabular}{|c|c|c|c|c|c|}
\hline Introductic & & & & & \\
\hline PARK12 & Classical PD & Risk factor & unknown & Unknown & $\begin{array}{l}\text { Confirmed } \\
\text { susceptibility locus }\end{array}$ \\
\hline PARK13 & Classical PD & $\begin{array}{l}A D \text { or risk } \\
\text { factor }\end{array}$ & Omi/HtrA2 & Unknown & Unconfirmed \\
\hline PARK14 & $\begin{array}{l}\text { Early-onset } \\
\text { dystonia- } \\
\text { parkinsonism }\end{array}$ & $\mathrm{AR}$ & PLA2G6 & Lewy bodies & Confirmed \\
\hline PARK15 & $\begin{array}{l}\text { Early-onset } \\
\text { parkinsonian- } \\
\text { pyramidal } \\
\text { syndrome }\end{array}$ & AR & FBXO7 & Unknown & Confirmed \\
\hline PARK16 & Classical PD & Risk factor & unknown & Unknown & $\begin{array}{l}\text { Confirmed } \\
\text { susceptibility locus }\end{array}$ \\
\hline PARK17 & Classical PD & $A D$ & VPS35 & Unknown & Confirmed \\
\hline PARK18 & Classical PD & $A D$ & EIF4G1 & Unknown & Unconfirmed \\
\hline PARK19 & $\begin{array}{l}\text { Early-onset } \\
\text { parkinsonism }\end{array}$ & AR & DNAJC6 & Unknown & Unconfirmed \\
\hline PARK20 & $\begin{array}{l}\text { Early-onset } \\
\text { dystonia- } \\
\text { parkinsonism } \\
\text { with cognitive } \\
\text { decline }\end{array}$ & AR & SYNJ1 & Unknown & Unconfirmed \\
\hline
\end{tabular}

\subsection{Rodent models of Parkinson's disease}

Novel insight into the mechanisms behind neurological disorders has come from the use of animal models, which are also prerequisites for development of new treatment strategies and evaluation of their efficacy. The focus lies on rodent models as they are closely related to humans. A good animal model of Parkinson's disease should capture the features of PD in humans such as slow onset, loss of dopaminergic neurons and consequently the reduction in dopamine levels. The model should also allow for testing the response to current pharmacological treatment.

\subsubsection{Pharmacological models}

For Parkinson's disease the first models developed were toxin models. A group of drug abusers in 1986 California suddenly developed full-blown Parkinson's after intake of 1methyl-4-phenyl-1,2,3,6-tetrahydropyridine (MPTP), a by-product of heroin synthesis (Davis et al., 1979). The metabolite of this compound, MPP+, is a specific blocker of complex I of the mitochondrial electron transport chain (Trevor et al., 1988). Administration of this drug in mice, especially in the C57BL/6 strain, gives rise to motor symptoms and loss of dopaminergic neurons similar to PD (Donnan et al., 1987; Sonsalla and Heikkila, 1988). The pesticide rotenone is also a blocker of complex I activity in mitochondria, but in contrast to MPTP, it induces systemic mitochondrial defects (Xiong et al., 2012). MPTP and rotenone function very differently in different genetic backgrounds and the effects are also sometimes spontaneously reversible (Sedelis et al., 2001). This 
prompted the use of the 6-hydroxy dopamine (6-OHDA) as a selective toxin of dopaminergic neural terminals when injected in the striatum. Mice treated in this way show long-lasting motor effects on top of dopaminergic neuron loss (Alvarez-Fischer et al., 2008).

These models have in common that they represent a tool to investigate acute onset of symptoms, but might fail to model the slowly progressing cellular defects that underlie PD.

\subsubsection{Knock-in mouse models}

To model the impending cellular breakdown, researchers have taken advantage of information from genetic PD cases. Initially, mouse lines overexpressing either wild type human $\alpha$-synuclein or mutant versions were generated. Several of these lines have successfully recapitulated the formation of Lewy body-like inclusions containing $\alpha$ synuclein accompanied by a progressive neurodegenerative phenotype (Chesselet et al., 2012; Martin et al., 2006; Rockenstein et al., 2002). These lines, however, use panneuronal promoters and the pathology is not restricted to dopaminergic neurons. When SNCA was expressed under the chatecholaminergic tyrosine hydroxylase (TH) promoter, pathology was absent (Matsuoka et al., 2001). The symptoms showing in the former lines could be attributed to inappropriately high expression of $\alpha$-synuclein in neurons and nuclei of the brainstem and spinal cord including motor neurons, hence explaining why no nigral loss is present. One reason why such over-expression models might not be suitable is that rodents handle aggregations differently from humans. Overexpression of APP, normally thought to cause Alzheimer's plaques in humans, fail to cause protein aggregations in mice until very late, which could be due to a protective enzymatic cascade (Stein and Johnson, 2002). Also the a-synuclein A53T mutation, which causes PD in humans, is the wild type mouse variant.

LRRK2 mouse models have similar to SNCA used a knock-in approach with mutations found in patients. Mice with both overexpression of the human R1441G and G2019S mutants displayed motor deficits, with the latter model even showing dopaminergic neuronal loss ( $\mathrm{Li}$ et al., 2009b; Ramonet et al., 2011). This is controversial however, as several other lines failed to show any loss of dopaminergic neurons (Lin et al., 2009; Melrose et al., 2010).

\subsubsection{Knockout mouse models}

Recessively inherited PARK syndromes are thought to be best modeled by knockout lines that mimic a loss-of-function mechanism. The most common variant, PARK2 (parkin), has been modeled in several knockout lines, but none have shown significant motor 
symptoms or neuronal loss (Goldberg et al., 2003; Perez and Palmiter, 2005; Von Coelln et al., 2004). The same is true for PINK1 knockout models (Kitada et al., 2007). Several DJ-1 knockout models have also been created, but only recently has one been shown to have clear dopaminergic neuron loss (Rousseaux et al., 2012). The results remain controversial, as only a subset of the animals showed neuronal loss, potentially due to other modulatory genetic factors. Notably, a triple knockout of all three genes showed neither a motor phenotype nor loss of neurons (Kitada et al., 2009).

\subsubsection{Novel animal models of PD}

Recently, novel $\alpha$-synuclein models have been developed that take advantage of both adeno-associated viruses and lentiviral vectors to overexpress $\alpha$-synuclein in the substantia nigra (Fischer et al., 2016). The initial problems with these models were that the load and targeting of the vectors gave highly variable results. However, the technique has been developing rapidly, and models that show (i) initial axon terminal dysfunction, (ii) progressive nigrostriatal degeneration, (iii) neuroinflammation and (iv) robust motor phenotypes have been characterized (Chung et al., 2009; Decressac et al., 2012; Gombash et al., 2013; Lundblad et al., 2012).

Another interesting strategy for $\alpha$-synuclein based murine PD models is based on the finding that once the $\alpha$-synuclein-preformed fibrils (PFFs) appear, they can spread in a prion-like fashion in vivo. Mice inoculated with PFFs of recombinant mouse a-synuclein showed a progressive formation of aggregations in a subset of neurons accompanied by reduced motor function and dopaminergic neuron death (Luk et al., 2012). Building on this notion, researchers have developed an interesting new approach where a-synuclein aggregates are administered intranasally. This model shows rapid onset of motor defects and avoids stressful animal handling, but is still new and further characterization is needed (Gruden et al., 2014).

\subsection{Molecular mechanisms of Parkinson's disease}

The molecular mechanisms underlying PD are heavily debated and several questions need answers. Firstly, what underlies the selective loss of dopaminergic neurons? The current hypothesis suggests that dopaminergic neurons are more vulnerable due to high metabolic rates and exposure to toxic oxidative by-products of dopamine metabolism. Additionally, most sporadic Parkinson's patients show Lewy body pathology. But are these proteinaceous deposits a cause or a consequence of cellular dysfunction? It has recently been shown that $\alpha$-synuclein aggregates can "infect" healthy neurons in a prion-like 
manner, suggesting an extracellular propagation mechanism (Olanow and Brundin, 2013). This is confounded by the fact that not all Parkinson's patients show heavy Lewy body pathology and that familiar variants largely lack this hallmark. And lastly, although it is clear that dopaminergic neurons are lost, this loss is often subsequent to a loss of dopamine release. Hence, what is the sequence of events leading to dopaminergic cell death and which mechanisms are responsible?

\subsubsection{Synaptic dysfunction}

The loss of dopamine release seen in PD patients suggests a failure of the synaptic machinery. This notion is supported by a-synuclein being present at synapses (Cheng et al., 2011) and involved in neurotransmitter release (Nemani et al., 2010). Particularly, $\alpha-$ synuclein is thought to be involved in vesicle formation and release. This is partially conveyed by binding to dopaminergic vesicles directly, and partially by interaction with phospholipase D2, an enzyme that stimulates recruitment of adaptor molecules upon neurotransmitter activation (Lotharius and Brundin, 2002). In addition, LRRK2 has been found to phosphorylate proteins involved in vesicle recycling (Matta et al., 2012; Piccoli et al., 2011; Yun et al., 2013). A dysfunction in these proteins might lead to a failure of vesicle release and synaptic dysfunction. This might again trigger a so-called dying-back mechanism hypothesized by multiple studies, in which synaptic dysfunction triggers axonal terminal loss and subsequently lead to neuronal cell death. An early observation in a human morphological study supporting this view shows that loss of axon terminals in the striatum is more pronounced than dopaminergic neuron loss in the substantia nigra (Bernheimer et al., 1973). Later investigation has revealed early axonal dysfunction both in chronic and acute MPTP rodent models (Li et al., 2009a; Serra et al., 2002). Also in some of the viral vector $\alpha$-synuclein models, axonal pathology was seen prior to dopaminergic neuronal loss causing the authors to hypothesize that the dying-back mechanism is important for $\alpha$-synuclein pathology (Chung et al., 2009; Decressac et al., 2012).

\subsubsection{Mitochondrial dysfunction}

Mitochondrial dysfunction has been shown in brains of PD patients in several studies (Keeney et al., 2006; Parker et al., 1989; Schapira et al., 1989). Although PD is characterized by the loss of dopaminergic neurons, mitochondrial defects have been observed in other brain areas as well as in skeletal muscles and platelets of PD patients (Bindoff et al., 1991; Haas et al., 1995). The connection between mitochondrial impairment and $\mathrm{PD}$ was further strengthened by the discovery of toxic compounds causing PD. MPTP, the first toxin to be associated with PD, is metabolized to MPP+, 
which resembles dopamine and is taken up by dopaminergic neurons where it inactivates complex I of the electron transport chain in mitochondria (Trevor et al., 1988). The same is the case for rotenone, a pesticide that can be used to model PD in rats (Xiong et al., 2012). Interestingly, these pharmacological models of PD ties mitochondrial dysfunction to synaptic failure: MPTP-treated monkeys show destruction of striatal terminals before cell body loss (Herkenham et al., 1991), and MPTP-treated mice show reduced neuronal loss when the striatal terminal degradation is prevented (Wu et al., 2003). Additional line of evidence for mitochondrial involvement stems from research done on parkin, PINK1 and DJ-1. These proteins are involved in clearance of dysfunctional mitochondria and mutations in either gene cause early-onset PD. Healthy mitochondria can maintain a stable membrane potential that causes PINK1 to be transported into the mitochondrial lumen and proteolysed. On the other hand, when this potential is lost, PINK1 is stabilized on the outer membrane where it functions to recruit parkin (Ziviani et al., 2010). Parkin is an E3 ubiquitin ligase that subsequently ubiquitinates several proteins associated with the mitochondrial membrane and targets these for destruction by the autophagosome (Gegg et al., 2010). DJ-1 is predominantly involved in protecting cells from oxidative stress (Taira et al., 2004; Yokota et al., 2003) and PD patients have shown an increase in oxidized proteins in the brain (Alam et al., 1997). DJ-1 is also localized to the mitochondria and has been shown to induce mitochondrial damage in a knockout mouse model (Krebiehl et al., 2010). Taken together, there are strong indications that mitochondrial dysfunction is heavily involved in PD pathology.

\subsubsection{Protein clearance}

In addition to the mitochondria, another of the cells' crucial regulatory systems, the ubiquitin proteasome system (UPS) has been investigated as a potential PD mechanism. Firstly, Lewy bodies are proteinaceous deposits that consist of many proteins, and notably both ubiquitin and proteasomal subunits have been found amongst them (Forno, 1996; li et al., 1997). Aside from classical Lewy bodies, protein aggregation has been observed in the substantia nigra of Parkinson's patients (Lopiano et al., 2000) and proteasomal activity seems to be decreased in PD patients compared to healthy control individuals (McNaught and Jenner, 2001). Although the composition of the proteasome seems normal (Furukawa et al., 2002), selective decrease in $20 \mathrm{~S}$ proteasome function has been found in the substantia nigra (SN) of PD patients (McNaught et al., 2002a). Also, systemic administration of proteasome inhibitors was shown to cause Parkinsonian symptoms in mice (McNaught et al., 2004), but this study is controversial, as the findings have not been replicated in subsequent studies (Bove et al., 2006; Kordower et al., 2006; Manning-Bog et al., 2006). When the $26 \mathrm{~S}$ proteasome, however, was selectively disrupted through the 
knock-down of Rpt2 in mouse forebrain or tyrosine hydroxylase positive neurons, severe neurodegeneration was found (Bedford et al., 2008). In addition, a study aiming at identifying novel biomarkers for PD found elevated levels of proteasomal subunit $\alpha 2$ (PSMA2) in blood samples from PD patients compared to healthy individuals (Grunblatt et al., 2010).

One of the recently discovered PARK genes encodes UHCL1, a de-ubiquitylase responsible for replenishing the pool of monomeric ubiquitin required for marking protein for degradation (Pickart, 2000). Mutations in this protein in a gracile axonal dystrophy (GAD) mouse model led to inclusion body formation, which stained positive for ubiquitin (Saigoh et al., 1999). In addition, overexpression of human $\alpha$-synuclein leads to aggregation formation in mice (Masliah et al., 2000). The E3 ubiquitin ligase parkin (PARK2) has also been found to bind to the proteasome in a ligase independent manner and knockdown of parkin lowered proteasomal activity by 25\% (Um et al., 2010).

The accumulation of protein aggregates and proteasomal dysfunction in PD patients strongly suggest the UPS as a potential therapeutic target for PD and warrants further investigation.

\subsection{FBX07}

This study centers around one of the recently identified PARK genes, Fbxo7 (PARK15), which encodes an E3 ubiquitin ligase harboring an F-box motif (Cenciarelli et al., 1999; Winston et al., 1999).

\subsubsection{PARK15 patients}

Fbxo7 was first identified as a Parkinson-associated gene by a genome-wide linkage analysis in an Iranian family with Parkinsonian-Pyramidal syndrome (Shojaee et al., 2008). These patients presented with Babinski sign, spasticity and hyperactive reflexes as initial symptoms and later onset of extra-pyramidal symptoms like rigidity and bradykinesia, but no signs of tremor. The disease-causing mutation was found to be the missense mutation Arg378Gly. Two other families, one Italian and one Dutch, were then found to carry three other mutations in Fbxo7; a nonsense mutation (Arg498X) and a compound heterozygous mutation involving an internal splice site (IVS7+ 1G/T and Thr22Met) (Di Fonzo et al., 2009). The affected individuals in these families presented with early-onset Parkinsonism as their first symptoms and later pyramidal signs similar to the ones seen in the Iranian family. FBXO7 was on grounds of these findings proposed as 
a Parkinson-associated gene and the syndrome named PARK15. Since then, patients carrying mutations in Fbxo7 has been found in a Chinese cohort study (Lin et al., 2013), and in several patients from Pakistan and Turkey (Conedera et al., 2016; Gunduz et al., 2014; Hanagasi et al., 2007; Lohmann et al., 2015; Paisan-Ruiz et al., 2010; YalcinCakmakli et al., 2014). A summary of mutations and symptoms of PARK15 patients is found in the appendix (Table A1). Parkinsonian symptoms were alleviated in all patients treated with L-dopa, but dyskinesia and behavioral disturbances were frequent side effects and treatment often discontinued. In some patients general brain atrophy was found upon MRI scanning and one patient showed a diffuse CIT-SPECT, suggesting presynaptic disturbances in the striatum (Di Fonzo et al., 2009). In most families Parkinsonian features were prominent, while in the Iranian family some patients only showed pyramidal signs. PARK15 is therefore classified as a Parkinsonian-Pyramidal Syndrome likely involving multiple brain regions.

\subsubsection{The ubiquitin proteasome system}

E3 ubiquitin ligases such as FBXO7 are part of the ubiquitin proteasome system, which is mainly responsible for protein degradation (Hershko and Ciechanover, 1998). To ensure protein homeostasis in the cell, it is crucial to have a degradation system that recognizes proteins to be destroyed at the right time and place. Proteins are marked for degradation by ubiquitin, a small molecule of $8.5 \mathrm{kDa}$ that is covalently attached to lysine residues of the target substrate. Three enzymes conduct this procedure; firstly the E1 ubiquitinactivating enzyme activates ubiquitin by binding to a sulfide group in an ATP-dependent manner, then the E2 ubiquitin-conjugating enzyme carries the ubiquitin to the E3 ubiquitin ligase. Here, the substrate is brought into close contact with the E2-ubiquitin conjugate from where ubiquitin is transferred onto the substrate (Figure 1.1).

Substrates ubiquitinated by lysine (K)48 chains are mostly recognized and degraded by the proteasome (Figure 1.1). These are multi-protein organelles consisting of a barrelshaped core with two outer alpha rings and two inner beta rings (Bochtler et al., 1999). Three of the beta subunits harbor different catalytic properties termed the chymotrypsinlike $(\beta 5)$, trypsin-like $(\beta 2)$ and post-glutamylpeptide hydrolysing or caspase-like ( $\beta 1)$ activity (Groll et al., 2005). The alpha-rings confer structural stability and the N-termini of the alpha-ring normally extend into the pore, keeping the proteasome closed when the regulatory particle is not bound. The regulatory particle binds to the alpha-rings and consist of a base and a lid (Tomko and Hochstrasser, 2013). The base has six AAAATPase subunits (Rpt1-6) responsible for the energy-consuming process of unfolding and transfer of the targeted proteins into the proteolytic chamber. It also has two large non- 
ATPase subunits, Rpn 1 and 2, and is bound to the lid by Rpn10 and 13 . Rpn10 and 13 might also function as substrate recognizing subunits. The lid consists of eight nonATPase subunits (Rpn3, 5-9, 11 and 12). In addition to harboring substrate recognition motifs, the proteasome transiently binds to interacting particles. Such particles, including Rad23, harbor ubiquitin-recognition motifs as well as ubiquitin-like domains allowing the recruitment of poly-ubiquitinated substrates to the proteasome.

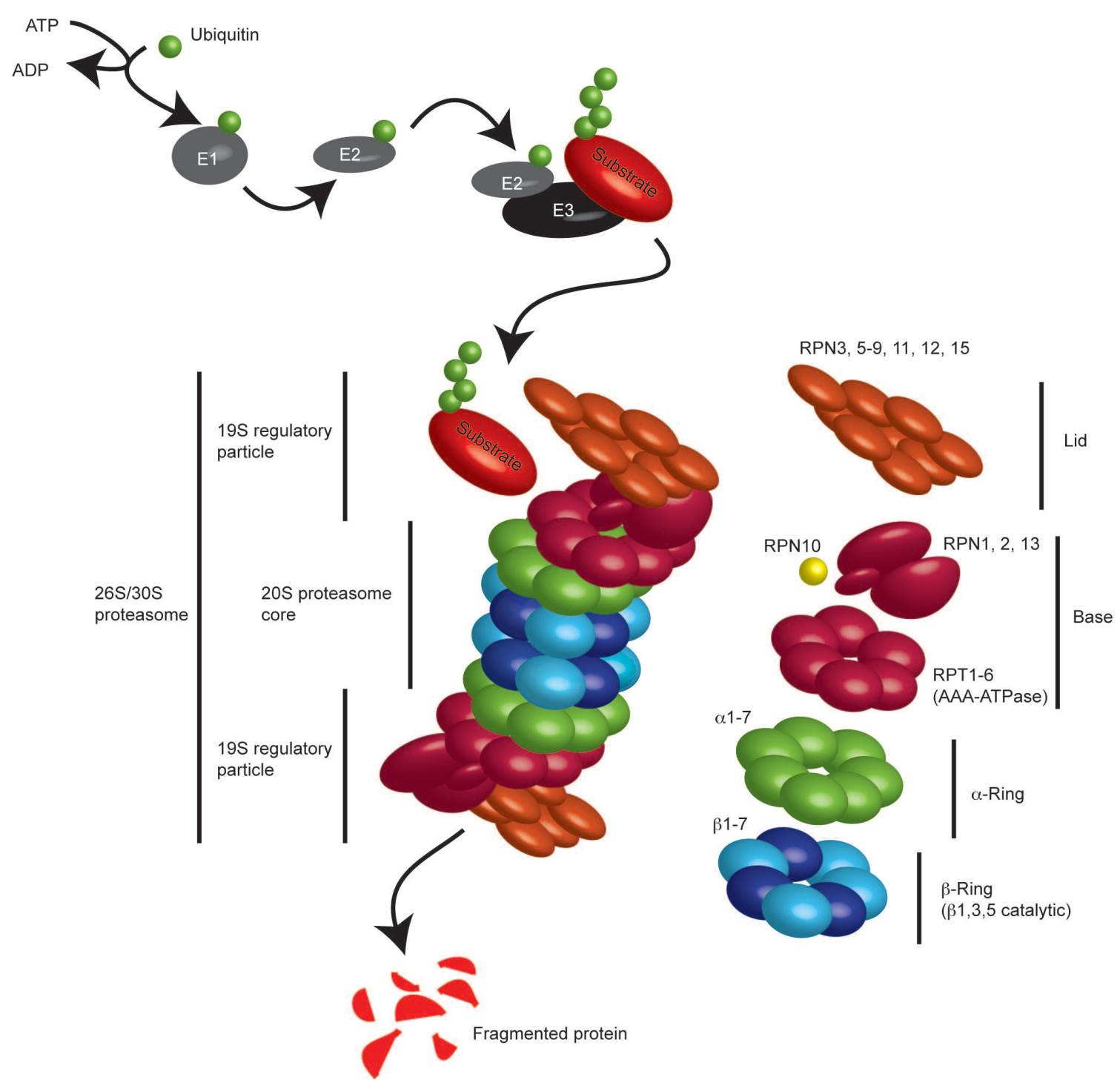

Figure 1.1 Schematic of the ubiquitin proteasome system. Ubiquitination of target substrates mediated by E1 ubiquitin-activating enzyme, E2 ubiquitin-conjugating enzyme and E3 ubiquitin ligase lead to degradation of the substrate by the $26 \mathrm{~S} / 30$ S proteasome.

\subsubsection{E3 ubiquitin ligases}

The enzyme responsible for conferring specificity to the protein degradation process is the E3 ubiquitin ligase. While 2 E1 enzymes and 30 E2 enzymes are currently known 
(Scheffner et al., 1995), more than 600 E3 ligases have been identified, which provide extensive control mechanisms of protein regulation (Deshaies and Joazeiro, 2009). An E3 ligase recognizes individual substrates through specific interaction motifs. One E3 ligase can have various substrates, and one substrate can be recognized by more than one ligase giving a certain degree of redundancy and thus flexibility to the system. Ubiquitin itself has seven lysines $(\mathrm{K})$, upon which ubiquitination chains can be attached. Chains formed via K48 attachment are more commonly associated with proteasomal degradation, while chains formed on a K63 base are known for a variety of functional modification (Pickart, 2004). The other chain types, K6, K11, K27, K29 and K33 are not well characterized yet (Ikeda and Dikic, 2008).

Classically two main types of E3 ligases are found: the homologous to C-type (HECT) and the really interesting new gene (RING) type (Pickart and Eddins, 2004). The HECT-type harbors intrinsic enzymatic activity and the ubiquitin is transferred from the E2 to the E3 before ligation to the target substrate (Kamadurai et al., 2013). The RING-type acts as a scaffold protein, mediating interaction between the E2 and the substrate. FBXO7, and other RING type E3 ligases, typically form multi-subunit enzymes, one of the most common ones being the Skp1-Cullin1-F-box protein (SCF) complex (Cardozo and Pagano, 2004) (Figure1.2). F-box proteins are responsible for target recognition and comprise a wide group of proteins harboring a so-called F-box motif, which facilitates the association with the core components S-phase kinase-associated protein 1 (Skp1) and cullin1. They are further subdivided according to their additional motifs; FBXW, which has a WD-40 motif, FBXL, with leucine-rich repeats at the C-terminus and FBXO, which have other motifs (Jin et al., 2004). The other essential partner of the SCF complex is Ring-box $1(\mathrm{Rbx} 1)$, the RING protein that facilitates interaction with the E2-ubiquitin conjugate.

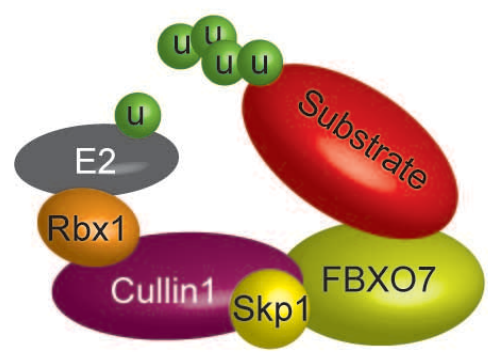

Figure 1.2 Schematic representation of the Skp1-cullin1F-box protein (SCF) complex. Skp1=S-phase kinaseassociated protein 1; Rbx1=Ring-box 1.

\subsubsection{Interaction partners of FBXO7}

FBXO7 was identified together with other F-box proteins in 1999 through a yeast twohybrid screen using Skp1 as bait (Cenciarelli et al., 1999; Winston et al., 1999). Here, FBXO7 was shown to bind cullin1, Skp1 and Rbx1 to form an SCF complex harboring 
ligase activity. The SCF-FBXO7 complex was later found to be the fifth most abundant SCF complex in HEK 293 cells (Lee et al., 2011). As a part of an SCF complex, FBXO7 is expected to interact with target proteins to ubiquitinate them, but so far not many ubiquitination targets have been characterized. Three isoforms of FBXO7 has been identified; isoform two lacks the first 25 amino acids, while isoform three has a different $\mathrm{N}$ terminal structure. Interestingly, although isoform three has never been shown to be expressed as a protein, a common SNP Met115lle changes its start codon, suggesting that this group of people completely lack isoform three (Nelson et al., 2013).

The first FBXO7-interacting protein to be found, and one of the few so far confirmed as a target of ubiquitination, is hepatoma up-regulated protein (HURP) (Hsu et al., 2004) (Figure 1.3). The ubiquitination of HURP was conveyed by the $\mathrm{N}$-terminal proline-rich repeat (PRR) of FBXO7 and depended upon HURP-phosphorylation by the Cyclin B/Cdk1 complex. HURP is a cell-cycle regulated protein that shows elevated expression during the $G_{2} / M$ phase and localizes to the spindle poles during mitosis (Tsou et al., 2003). In addition to being up-regulated in hepatomas, elevated gene expression of HURP is associated with colon cancer, breast cancer and transitional cell carcinoma (Bassal et al., 2001; Chiu et al., 2002; Huang et al., 2003).

In concordance with this finding, it was later shown that FBXO7 also interacts specifically with other proteins involved in cell-cycle regulation, namely p27 and the Cyclin D/Cdk6 complex (Laman et al., 2005) (Figure 1.3). Cyclin-dependent kinases are activated by D cyclins to transform the information from growth signals into cell cycle progression (Morgan, 1995; Sherr, 1996). Knockdown of FBXO7 reduced the levels of Cyclin D/Cdk6 assembly factors, suggesting that $\mathrm{FBXO} 7$ might be a positive regulator of these proteins in a ligase independent manner. The binding of FBXO7 to Cdk6 was independent of its Fbox domain but this domain significantly increased the transforming effect of FBXO7 overexpression in immortalized murine fibroblasts. In addition, it was shown that FBXO7 injection into nude mice caused cell transformation and tumor formation (Laman et al., 2005).

P27 regulates Cyclin D/Cdk complexes by facilitating their stabilization, nuclear import and nuclear retention (Alt et al., 2002; Cheng et al., 1999; LaBaer et al., 1997). FBXO7 was found to directly interact with p27 (Figure 1.3), but this interaction did not affect its facilitation of Cyclin D/Cdk6 assembly. A later study showed that $p 27$ levels were reduced in a partial FBXO7 knockout mouse, suggesting that FBXO7 stabilizes p27, thus promoting cell cycle withdrawal (Randle et al., 2015). 


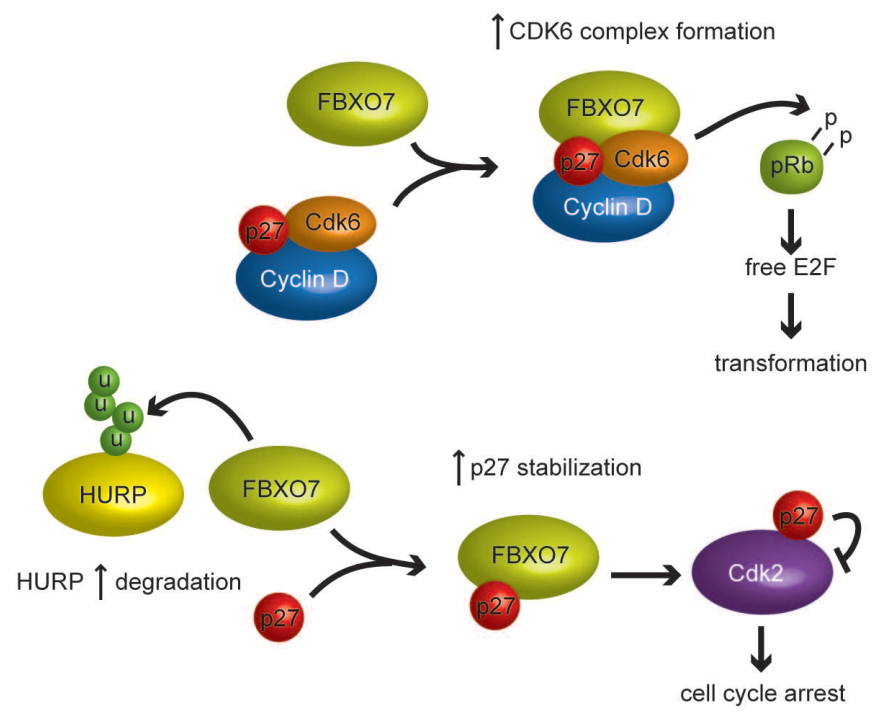

Figure 1.3 FBXO7 is a cell cycle regulator. FBXO7 can ubiquitinate the cell cycle regulator HURP, leading to its degradation. In addition, it can stabilize p27 and lead to an increase in formation of Cyclin D/Cdk6 complexes with opposing effects on cell cycle. Modified from Nelson et. al. (Nelson et al., 2013).

In human tissue, FBXO7 was found to be absent from colon and lung tissue in healthy controls, but present in carcinomas from the same tissue in cancer patients, thus further implicating FBXO7 in cancer biology (Laman et al., 2005).

In contrast to the findings in fibroblasts, it was shown that lack of FBXO7 leads pro-B cells into accelerated proliferation with a shortened G1 phase and a decrease in cell size (Meziane el et al., 2011). This is accompanied by genome-wide analyses studies showing the correlation of SNPs in the FBXO7 gene with changes in red blood cell parameters (Ding et al., 2012; Soranzo et al., 2009; van der Harst et al., 2012). The partial FBXO7 knockout mouse also presented with signs of prolonged anemia and increased number of pro-beta cells in their spleens (Laman et al., 2005; Randle et al., 2015).

Apart from HURP, two other proteins have been found to be direct targets of FBXO7mediated ubiquitination, namely cellular inhibitor of apoptosis 1 (cIAP1) and TNF receptorassociated factor 2 (TRAF2) (Chang et al., 2006; Kuiken et al., 2012). These proteins are both ubiquitin ligases involved in NF-KB signaling, although TRAF2 might act as a scaffold rather than a ligase in this process (Mahoney et al., 2008). The ubiquitination of receptorinteracting protein 1 (RIP1) downstream of these ligases lead to a cascade where I $\mathrm{kB}$ is targeted for degradation and NF-kB can translocate to the nucleus and induce transcription of a wide array of genes, including those involved in immune responses (Chen and Goeddel, 2002). FBXO7 was found to ubiquitinate both TRAF2 and cIAP1, 


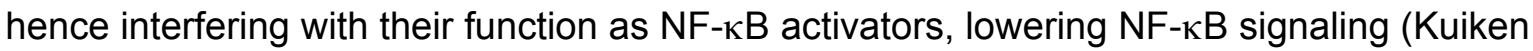
et al., 2012) (Figure 1.4). Whether this is proteolytic ubiquitination or a functional modification remains to be elucidated, but another study suggest that ubiquitination of TRAF2 targets it for degradation (Chen et al., 2014). An alternative function for FBXO7 in $\mathrm{NF}-\kappa \mathrm{B}$ pathway is through its interaction with neurotrophin receptor-interacting MAGE protein (NRAGE). FBXO7 was found to ubiquitinate NRAGE via K63 linked chain and hence activate NF- $\mathrm{KB}$ signaling through its alternative bone morphogenic protein (BMP4) pathway (Kang and Chung, 2015; Matluk et al., 2010).

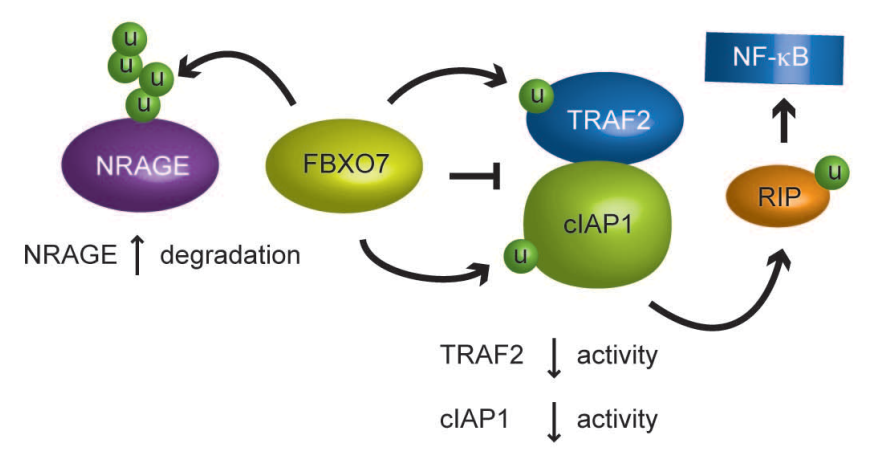

Figure 1.4 FBXO7 regulates NF-KB signaling. FBXO7 ubiquitinates three proteins involved in NF- $\kappa B$ signaling leading to a reduction in the traditional TNF signaling pathway and an increase in the alternative BMP pathway. Modified from Nelson et al. (Nelson et al., 2013).

Two interesting interaction partners of FBXO7 in the PD context are parkin and PINK1 (Burchell et al., 2013). FBXO7 seems to be involved in the translocation of parkin to depolarized mitochondria and the subsequent ubiquitination of Mfn1 (Figure 1.5). It is however not clear what role FBXO7 plays in mitochondrial quality control.

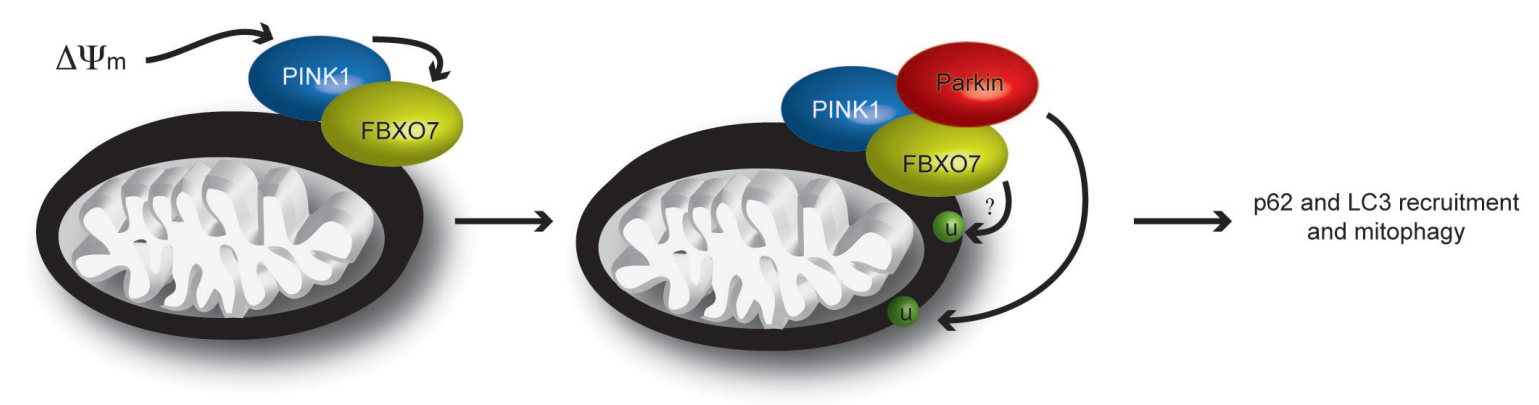

Figure 1.5 FBXO7 is involved in mitophagy. PINK1 recruits FBXO7 to mitochondria with subsequent recruitment of parkin and ubiquitination of mitochondrial membrane proteins. Modified from Nelson et al. (Nelson et al., 2013). 


\subsubsection{Proteasomal inhibitor 31 (PI31)}

Given the role of proteasomal dysfunction in PD, proteasomal inhibitor 31 (PI31) is an interesting interacting partner of FBXO7. PI31 was first identified in a screen for regulators of the 20 s proteasome core activity (Chu-Ping et al., 1992) and shown to be an in vitro inhibitor of the caspase-like activity of the 20 S core, competing with PA28 for binding to the proteasome (McCutchen-Maloney et al., 2000; Zaiss et al., 1999). A subsequent study, however, could not find that PI31 inhibits the 20S proteasome in vivo, but rather caused a defect in maturation of immunoproteasomes (Zaiss et al., 2002). This suggests that $\mathrm{PI} 31$ regulates the formation of immunoproteasomes in vivo.

An attractive hypothesis was proposed by Cho-Park and Stellar, where PI31 was shown to interact with the core as well as with the chaperones of the proteasome cap through ADP-ribosylation (Cho-Park and Steller, 2013). They suggest that PI31 is involved in assembly of the 20S core and 19S cap by binding the core and pry away the chaperones from the $19 S$ cap, facilitating the assembly of the $26 S$ functional proteasome. This finding could however, not be replicated by an independent group ( $\mathrm{Li}$ et al., 2014). Li et al. showed that knockdown or over-expression of PI31 did not affect proteasome function in intact cells. Therefore, despite the strong in vitro evidence of $20 \mathrm{~S}$ inhibition and $26 \mathrm{~S}$ activation, it is difficult to establish a role for PI31 in proteasomal function in vivo.

$\mathrm{PI} 31$ was identified as an interaction partner of FBXO7 in a yeast two-hybrid screen (Kirk et al., 2008). It was then showed that PI31 and FBXO7 shared a dimerization domain named the FBXO7-PI31 (FP) domain as well as a conserved proline-rich repeat (PRR) sequence at their $\mathrm{N}$-termini (Figure 1.6).

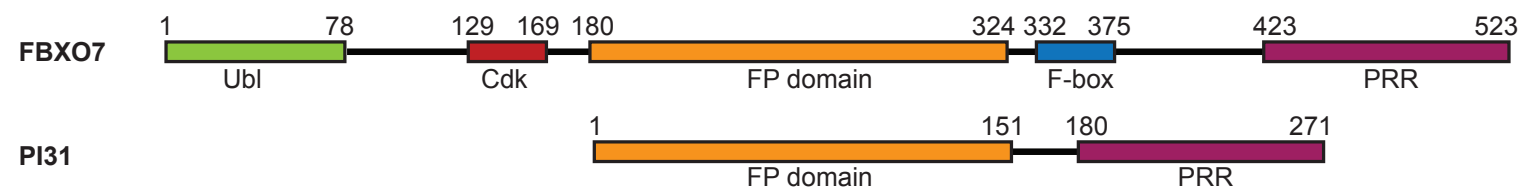

Figure 1.6 Domain structures of FBXO7 and PI31. FP domain=FBXO7 and PI31 interacting domain, $\mathrm{Cdk}=\mathrm{Cdk}$-interacting domain, $\mathrm{Ubl}=\mathrm{Ubiquitin}$-like domain, $\mathrm{PRR}=$ Proline-rich repeat domain.

Through the FP domain both homo and heterodimerization of the proteins are possible (Shang et al., 2015; Shang et al., 2014). It was also shown that FBXO7 could bind multiple proteins via its FP-domain and the possibility of multimers was proposed. In Drosophila melanogaster, PI31 was found to act as an activator of both 26S purified proteasomes and also in vivo boost proteasomal activity (Bader et al., 2011). Here, PI31 was also shown to interact with Nutcracker, a protein that shares some homology with 
FBXO7. This interaction was also found to stabilize PI31 protein levels trough a direct interaction mechanism. However, the mutant nutcracker flies show sterility, which could not be rescued upon FBXO7 expression suggesting that these are not functional homologues (Burchell et al., 2013). Consequently, the interaction mechanism between FBXO7 and PI31 is not fully investigated in mammalian cells and the importance of their neuronal interaction remains to be clarified.

Even though FBXO7 has been implicated in multiple cellular pathways, its function in neurons remains elusive, and makes it an interesting target for the study of PD disease mechanisms.

\subsection{Aim of the study}

Mutations in FBXO7 cause Parkinsonian-Pyramidal Syndrome (PARK15) in humans, but the mechanism underlying this disease is still unknown. To identify cellular pathways affected in PD we can take advantage of genetic data. In this study, I therefore set out to model PARK15 using a conventional and two conditional FBXO7 knockout mouse lines. In the conventional knockout, I sought to model the systemic loss of FBXO7, while in the conditional models I sought to investigate how brain-specific loss of FBXO7 impacted mice behavior and cellular function. With these models, I aimed at faithfully replicating Parkinsonian-Pyramidal syndrome in mice.

Based on FBXO7's interaction with PI31, I wanted to take advantage of cell lines and the mouse models to conduct biochemical experiments to elucidate FBXO7s role in proteasomal regulation. Taken together, I characterized FBXO7 knockout mice with the long-term aim of creating models for testing of therapeutic agents as well as neuronal tools to examine pathological pathways underlying Parkinsonian-Pyramidal syndrome. 
"A scientist in his laboratory is not a mere technician: he is also a child confronting natural phenomena that impress him as though they were fairy tales"

Marie Curie, "Madame Curie: A Biography"

\section{Materials and methods}

\subsection{Reagents and equipment}

\subsubsection{Chemicals}

Suppliers of chemicals for this study include: Sigma-Aldrich, Roth (Karlsruhe, Germany), Merck Millipore (Darmstadt, Germany), Applichem (Darmstadt, Germany), GE Healthcare (Little Chalfont, UK), Worthington (Lakewood, NJ, USA) or Th. Geyer (Hoexter, Germany). All chemicals were of either analytical purity or cell culture grade.

Cell culture media, PSG, GlutaMAX ${ }^{\mathrm{TM}}$ and Trypan Blue were purchased from Thermo Fisher Scientific (Waltham, MA, USA). Poly-L-ornithine hydrobromide as well as Mowiol mounting medium were acquired from Sigma-Aldrich (Munich, Germany), trypsin from Worthington, albumin fraction $V$ from Applichem and ECL western blotting substrates from Thermo Fisher Scientific (Waltham, MA, USA). Protein A-Sepharose and Gluthathionesepharose beads were ordered from GE Healthcare. Poly-L-Lysine (PLL) and goat serum were obtained from Sigma-Aldrich. Calf serum was purchased from HyClone (GE Healthcare), fetal bovine serum from Biochrom (Merck Millipore) and horse serum from PAA (Colbe, Germany). GelRed ${ }^{\mathrm{TM}}$ Nucleic Acid Gel Stain was bought from Biotium (Hayward, CA, USA) and Tissue-Tek ${ }^{\circledR}$ O.C.T Compund from Sakura Finetek (Torrance, CA, USA). Eukitt ${ }^{\circledR}$ mounting medium was obtained from O. Kindler (Freiburg, Germany).

\subsubsection{Enzymes}

Enzymes used in this study are listed in Table 2.1.1 along with their application.

Table 2.1.1 Enzymes used in this study

\begin{tabular}{lll}
\hline Enzyme & Reaction & Producer \\
\hline T4 DNA ligase & Ligation of DNA fragments & Fermentas (St. Leon-Rot, Germany) \\
$\begin{array}{l}\text { Pfu DNA polymerase } \\
\text { Calf intestinal alkaline } \\
\text { phosphatase }\end{array}$ & Polymerase chain reaction & Fermentas \\
Restriction enzymes & DNA digest & NEB (Frankfurt, Germany) \\
T4 polynucleotide kinase & $5^{\prime}$ ' phosphorylation & NEB \\
Proteinase K & Protein lysis & NEB \\
GoTaq DNA polymerase & Polymerase chain reaction & AppliChem \\
& &
\end{tabular}




\subsubsection{Kits}

Commercial kits used in this study are listed in Table 2.1.2. Manufacturers protocols were followed unless otherwise indicated.

Table 2.1.2 Commercial kits used in this study

\begin{tabular}{lll}
\hline Kit & Application & Producer \\
\hline NucleoSpin Plasmid kit & DNA isolation, small scale & Macherey-Nagel \\
NucleoBond Xtra Midi EF & DNA isolation, medium scale & Macherey-Nagel \\
NucleoSpin Extract II kit & DNA extraction and PCR Clean-Up & Macherey-Nagel \\
Pierce ECL substrate & Western blotting & Thermo Fischer \\
Bio-Rad Protein Assay & Protein quantification & Bio-Rad \\
PK-4000 peroxidase kit & DAB staining & Vector Laboratories \\
& & (Petersborough, UK) \\
SK-4100 kit & DAB staining & Vector Laboratories \\
SuperScript $\circledast$ III RT & cDNA synthesis & Thermo Fischer \\
SYBR $®$ green & Real Time PCR & Thermo Fischer \\
\hline
\end{tabular}

\subsubsection{Equipment and consumables}

The rotarod was bought from Ugo Basile (Ugo Basile, Comerio, Italy). Other equipment for behavioral testing was made by the fine mechanics department at the Max-Planck Institute of Experimental Medicine. Video surveillance system with Viewer software was purchased from BIOBSERVE (St. Augustin, Germany). Micropipettes were bought from Gilson (Limburg-Offheim, Germany), power suppliers and thermocycler from Biometra (Göttingen, Germany), heating blocks and centrifuges from Eppendorf, rocker and shaker from Heidolph (Schwabach, Germany) and electrophoretic apparatus from Bio-RAD (Munich, Germany). Cell culture safety hood and incubator, both HERAsafe ${ }^{\circledR}$, and infusion pump Heidolph ${ }^{\mathrm{Tm}}$ Pump Drive 5001, were from Thermo Scientific (Bonn, Germany). Fluorescent plate reader (Wallac 1420 VICTOR2 $^{\mathrm{TM}}$ ) was from Perkin Elmer (Waltham, MA, USA). Gel Imager for UV detection was from INTAS (Göttingen, Germany). Cryo-stat was from Leica (Wetzlar, Germany). Inverted light microscope (Eclipse TS100) and dissection microscope (SMZ645) were from Nikon (Tokyo, Japan), brightfield light microscope (Zeiss Axiophot) and flourescent microscope (Zeiss observer Z.1) from Zeiss (Oberkochen, Germany).

Consumables were purchased from Falcon (Becton Dickinson Labware Europe, Le Pont De Claix, France), Sarstedt AG (Nürnbrecht, Germany), Becton Dickinson (Heidelberg, Germany), Greiner Bio-One (Frickenhausen, Germany) and Eppendorf (Hamburg, Germany). 


\subsubsection{Software}

Software used in the study for image and video acquisition, data processing, quantification and statistical analyses is listed in Table 2.1.3.

Table 2.1.3 List of software used in this study

\begin{tabular}{lll}
\hline Software & Application & Source/Manufacturer \\
\hline NIS-Element & Image-acquisition and analysis & Nikon \\
ImageJ & Image analysis & http://rsbweb.nih.gov/ij/ \\
GraphPad Prism 6 & Statistical analyses & GraphPad Software, Inc. \\
Viewer tracking & Animal movement tracking & Biobserve, Germany \\
\hline
\end{tabular}

\subsection{Antibodies and vectors}

\subsubsection{Antibodies}

Primary antibodies used in this study are listed in Table 2.2.1 along with their manufacturer. Alexa fluor ${ }^{\circledR}$ 488, Cy3 and HRP-conjugated antibodies ( $\alpha$-mouse, $\alpha$-rat and a-rabbit lgG) were from Dianova (Hamburg, Germany), and a-goat IgG from Santa Cruz.

Table 2.2.1 Antibodies used in this study: IHC (Immunohistochemistry), WB (Western blot), rb: rabbit (affinity purified or serum), m: mouse (monoclonal), gt: goat (affinity purified)

\begin{tabular}{|c|c|c|}
\hline Target & Application & Reference \\
\hline m- $\alpha-F B X 07$ & WB (1:50) & Santa Cruz \\
\hline gt-a-PI31 & WB $(1: 500)$ & Sigma-Aldrich \\
\hline m- $\alpha-\gamma$-tubulin & WB (1:2000) & Sigma-Aldrich \\
\hline rb-a-PSMA2 & WB (1:2000) & Cell Signalling \\
\hline$m-\alpha-T H$ & IHC (1:1000) & Sigma-Aldrich \\
\hline rb- $\alpha-T H$ & IHC, WB (1:1000) & Zytomed \\
\hline$m-\alpha-G F A P$ & IHC (1:200) & Leica \\
\hline rb-a-lba1 & $\mathrm{IHC}(1: 1000)$ & WAKO \\
\hline m-a-Mac3 & IHC (1:200) & BD Pharmingen \\
\hline rat- $\alpha-D A T$ & WB (1:1000) & Millipore \\
\hline$m-\alpha-S P 1$ & WB $(1: 500)$ & Santa Cruz \\
\hline rb-a-VGLUT1 & WB (1:5000) & Synaptic Systems \\
\hline m- $\alpha-M y c$ & WB (1:2000) & Santa Cruz \\
\hline m-a-Flag & WB (1:2000) & Sigma \\
\hline m- $\alpha-N e u N$ & IHC (1:1000) & Millipore \\
\hline m-a-pan 14-3-3 & WB (1:2000) & Santa Cruz \\
\hline m- $\alpha$ - $\beta$-galactosidase & WB $(1: 500)$ & Santa Cruz \\
\hline
\end{tabular}




\subsubsection{Vectors}

Myc-tagged Psma2, Fbxo7 (cloned by Dr. Madhuvanthi Kannan), FP deletion mutant and point mutation variants were cloned into the pCMV-myc vector. Flag-tagged Pi31 (cloned by Dr. Judith Stegmüller) and Pi31 V83E/I90E mutant were cloned into the p3xFLAGCMV-10 vector. GFP-tagged Psma2 was cloned into the pEGFP-C1 vector (cloned by David Brockelt). shRNA were cloned into the pSUPER vector and the GST-Rad23B was cloned into the pGEX vector (cloned by David Brockelt).

\subsection{Buffers and Solutions}

The various buffers and solutions used in this study are listed in Table 2.3.1.

Table 2.3.1 Buffers and solutions used in this study

\begin{tabular}{|c|c|}
\hline Buffers and solutions & Ingredients \\
\hline 10X Phosphate buffer saline & $1.37 \mathrm{M} \mathrm{NaCl}, 14.7 \mathrm{mM} \mathrm{KCl}, 78.1 \mathrm{mM} \mathrm{Na}{ }_{2} \mathrm{HPO}_{4}, 26.8 \mathrm{mM}$ \\
\hline (PBS) & $\mathrm{KH}_{2} \mathrm{PO}_{4}, \mathrm{pH} 7.4$ \\
\hline PBST & 1xPBS, $0.1 \%$ Tween-20 \\
\hline Triton $^{\mathrm{TM}} \mathrm{X}-100$ Lysis Buffer & $\begin{array}{l}150 \mathrm{mM} \mathrm{NaCl}, 50 \mathrm{mM} \text { Tris-HCl pH 7.5, } 1 \mathrm{mM} \text { EDTA, } 1 \% \\
\text { Triton }^{\mathrm{TM}} \mathrm{X}-100\end{array}$ \\
\hline Co-IP buffer & $\begin{array}{l}150 \mathrm{mM} \mathrm{NaCl}, 20 \mathrm{mM} \text { Tris-HCl pH 7.4, } 1 \mathrm{mM} \text { EDTA, } 1 \% \\
\text { Nonidet P-40, } 10 \% \text { glycerol (freshly added } 3 \mu \mathrm{g} / \mathrm{ml} \\
\text { aprotinin, } 1 \mu \mathrm{g} / \mathrm{ml} \text { leupeptin, } 1 \mathrm{mM} \text { DTT and } 1 \mu \mathrm{g} / \mathrm{ml} \\
\text { pepstatin). }\end{array}$ \\
\hline 2 XYT media & $10 \mathrm{~g} / \mathrm{L}$ Yeast, $16 \mathrm{~g} / \mathrm{L}$ Tryptone, $5 \mathrm{~g} / \mathrm{L} \mathrm{NaCl}$ \\
\hline $2 \mathrm{xYT}$ agar plates & $\begin{array}{l}2 \times Y T \text { with } 1.5 \% \text { agar and either } 50 \mu \mathrm{g} / \mathrm{ml} \text { Ampicilin or } \\
\text { Kanamycin }\end{array}$ \\
\hline Running buffer & $25 \mathrm{mM}$ Tris base, $190 \mathrm{mM}$ glycine, $0.1 \%$ SDS \\
\hline Transfer buffer & $20 \mathrm{mM}$ Tris base, $153 \mathrm{mM}$ glycine, $20 \%$ methanol \\
\hline Upper buffer & $0.5 \mathrm{M}$ Tris- $\mathrm{HCl} \mathrm{pH} 6.8,0.4 \%$ SDS \\
\hline Lower buffer & $1.5 \mathrm{M}$ Tris- $\mathrm{HCl} \mathrm{pH} 8.8,0.4 \%$ SDS \\
\hline 5x SDS-sample buffer & $\begin{array}{l}300 \mathrm{mM} \text { Tris- } \mathrm{HCl} \mathrm{pH} 6.8,10 \% \text { SDS, } 50 \% \text { glycerol, } 25 \% \beta- \\
\text { mercaptoethanol, } 0.05 \% \text { bromophenol blue }\end{array}$ \\
\hline Annealing buffer & $\begin{array}{l}100 \mathrm{mM} \mathrm{CH}{ }_{3} \mathrm{COOK}, 30 \mathrm{mM} \text { HEPES-KOH } \mathrm{pH} 7.4,2 \mathrm{mM} \\
\left(\mathrm{CH}_{3} \mathrm{COO}\right)_{2} \mathrm{Mg}\end{array}$ \\
\hline 2x HBSS buffer & $\begin{array}{l}10 \mathrm{mM} \mathrm{KCl}, 280 \mathrm{mM} \mathrm{NaCl}, 15 \mathrm{mM} \text { glucose, } 1.5 \mathrm{mM} \\
\mathrm{Na}_{2} \mathrm{HPO}_{4}, 50 \mathrm{mM} \text { HEPES pH 7.05-7.11 }\end{array}$ \\
\hline $2 \mathrm{TAE}$ & 80 mM Tris-acetate, 2 mM EDTA pH 8.5 \\
\hline
\end{tabular}


Mowiol-mounting medium

\begin{tabular}{|c|c|}
\hline HHGN & $\begin{array}{l}\text { 1x HBSS, } 2.5 \mathrm{mM} \text { HEPES pH 7.5, } 35 \mathrm{mM} \text { glucose, } 4 \mathrm{mM} \\
\mathrm{NaHCO}_{3}\end{array}$ \\
\hline Proteasomal lysis buffer & $\begin{array}{l}50 \mathrm{mM} \text { Tris- } \mathrm{HCl} \mathrm{pH} 7.5,250 \mathrm{mM} \text { sucrose, } 5 \mathrm{mM} \mathrm{MgCl}_{2} \text {, } \\
2 \mathrm{mM} \text { ATP, } 1 \mathrm{mM} \text { DTT, 0.5mM EDTA, 0.025\% Digitonin }\end{array}$ \\
\hline Proteasomal activity buffer & $\begin{array}{l}50 \mathrm{mM} \text { Tris- } \mathrm{HCl} \mathrm{pH} \mathrm{7.5,40} \mathrm{mM} \mathrm{KCl,} 5 \mathrm{mM} \mathrm{MgCl}, 0.5 \mathrm{mM} \\
\text { ATP, } 1 \mathrm{mM} \mathrm{DTT}\end{array}$ \\
\hline Buffer A & $\begin{array}{l}10 \mathrm{mM} \text { HEPES, } 10 \mathrm{mM} \mathrm{KCl}, 0.1 \mathrm{mM} \text { EDTA, } 0.1 \mathrm{mM} \\
\text { EGTA, } 0.5 \mathrm{mM} \text { phenyl-methyl-sulphonyl fluoride (PMSF), } \\
1 \mathrm{mM} \text { dithiothrietol (DTT), } 5 \mu \mathrm{g} / \mathrm{ml} \text { aprotinin }\end{array}$ \\
\hline Buffer C & $\begin{array}{l}20 \mathrm{mM} \text { HEPES pH 7.9, } 400 \mathrm{mM} \mathrm{NaCl}, 1 \mathrm{mM} \text { EDTA, } 1 \mathrm{mM} \\
\text { EGTA, } 1 \mathrm{mM} \text { PMSF, } 1 \mathrm{mM} \text { DTT, } 5 \text { } \mathrm{gg} / \mathrm{ml} \text { aprotinin }\end{array}$ \\
\hline Genotyping buffer & $\begin{array}{l}200 \mathrm{mM} \mathrm{NaCl}, 10 \mathrm{mM} \text { Tris } \mathrm{pH} 8.0,10 \mathrm{mM} \text { EDTA, } 0.5 \% \\
\text { SDS, } 200 \mu \mathrm{g} / \mathrm{ml} \text { Proteinase } \mathrm{K}\end{array}$ \\
\hline TBS & $100 \mathrm{mM}$ Tris-Hcl pH 7.3, $154 \mathrm{mM} \mathrm{NaCl}$ \\
\hline Nissl stock staining solution & $1 \mathrm{~g}$ Thionine acetate in $100 \mathrm{ml} \mathrm{ddH_{2 } \mathrm { O }}$ \\
\hline Nissl buffer & $7 \mathrm{~g} \mathrm{C}_{2} \mathrm{H}_{3} \mathrm{NaO}_{2}, 2 \mathrm{ml} \mathrm{C}_{2} \mathrm{H}_{4} \mathrm{O}_{2}$ in 1 liter $\mathrm{H}_{2} \mathrm{O}$ \\
\hline Nissl working staining solution & $45 \mathrm{ml}$ stock staining solution, $455 \mathrm{ml}$ buffer \\
\hline CBC & $\begin{array}{l}\text { BME [+] Earle's salts [-] L-Glutamine, } 10 \% \text { calf serum } \\
\text { (heat inactivated), } 1 \% \text { PSG, } 25 \mathrm{mM} \mathrm{KCl}\end{array}$ \\
\hline TDn & $50 \mathrm{mg}$ Trypsin and $0.5 \mu \mathrm{g}$ DNase in $5 \mathrm{~mL} \mathrm{HHGN}$ \\
\hline DnB & $\begin{array}{l}0.4 \mu \mathrm{g} \text { DNase in } 4 \mathrm{~mL} \text { BME [+] Earle's salts [-] L- } \\
\text { Glutamine }\end{array}$ \\
\hline Mobile phase HPLC & $\begin{array}{l}6.9 \mathrm{~g} / \mathrm{l} \text { sodium acetate, } 48 \mathrm{mg} / \mathrm{I} \text { EDTA, } 7.3 \mathrm{~g} / \mathrm{l} \text { citric acid, } \\
105 \mathrm{mg} / \mathrm{l} \text { octane sulfonic acid and } 10 \% \text { methanol }(\mathrm{pH} 4.3)\end{array}$ \\
\hline
\end{tabular}

\subsection{Molecular cloning}

\subsubsection{Generation of short hairpin RNA}

A DNA-based template method, pSUPER RNAi System ${ }^{\mathrm{TM}}$, was used to express short hairpin RNAs against target sequences (Table 2.4.1) with high homology between mouse, rat and human (Brummelkamp et al., 2002; Saiki et al., 1985). 
Table 2.4.1 Target sequences for shRNA. bp=base pairs, hs=homo sapiens, mm=mus musculus.

\begin{tabular}{|c|c|c|c|}
\hline shRNA & $\begin{array}{l}\text { Target } \\
\text { region }\end{array}$ & $\begin{array}{l}\text { Nucleotides } \\
\text { identical/total }\end{array}$ & Sequence \\
\hline Fbxo7 shRNA\#1 & bp 178-197 & hs, mm, rat: $21 / 21$ & 5'-gaagagaccttggcttcata-3' \\
\hline Fbxo7 shRNA\#2 & bp 200-219 & hs, mm, rat: $21 / 21$ & 5'-ggattgtttctggggacttg-3' \\
\hline Fbxo7 shRNA\#ctrl & bp 1017-1037 & hs, mm, rat: $21 / 21$ & 5'-gaaactacgcatcttccgac-3' \\
\hline Psma2 shRNA\#1 & bp 179-199 & $\begin{array}{l}\text { hs: } 21 / 21 \text {, } \\
\text { mm: } 16 / 21 \text {, rat: } 18 / 21\end{array}$ & 5'-gaagtgtacacaaagtagaac-3' \\
\hline Psma2 shRNA\#2 & bp 680-700 & $\begin{array}{l}\text { hs: } 21 / 21 \text {; } \\
\text { mm, rat: } 20 / 21\end{array}$ & 5'-ggattacttggctgccatagc-3' \\
\hline Psma2 shRNA\#ctrl & bp 27-45 & hs, mm, rat: $21 / 21$ & 5'-gctgactacattcagccc-3' \\
\hline Pi31 shRNA\#1 & bp 261-280 & hs, mm, rat: $21 / 21$ & 5'-gggaagtggtgacaaacggct-3' \\
\hline Pi31 shRNA\#2 & bp 351-371 & $\begin{array}{l}\text { hs: } 19 / 21 \\
\text { mm, rat: } 21 / 21\end{array}$ & 5'-gaacagcaataaagaactgta-3' \\
\hline Pi31 shRNA\#ctrl & bp 969-988 & $\begin{array}{l}\text { hs: 19/21; } \\
\text { mm, rat: } 21 / 21\end{array}$ & 5'-gggctacgatgacatgtacc-3' \\
\hline
\end{tabular}

Primers for shRNA were designed by following criteria: Starts with GAA, GAG or GGG followed by 18 nucleotides, has $40-60 \%$ GC content and avoids AUG and AAAA. The loop sequence AAGTTAACG, which harbours an Hpal selection site, was implemented in between the forward and reverse primer sequence and the whole construct flanked by a Bglll and a HindIII cut site. The construct was then cloned into the pSUPER vector. Primers were resuspended in annealing buffer at $7 \mathrm{pmol}$ and incubated at $95^{\circ} \mathrm{C}$ for 4 minutes and then $72^{\circ} \mathrm{C}$ for 10 minutes and slowly cooled. The oligonucleotide mixture 2:5 was then phosphorylated with $1 \mu \mathrm{l} \mathrm{T4}$ polynucleotide kinase and $0.4 \mathrm{mM}$ ATP in $50 \mu \mathrm{l}$ total volume for 30 minutes at $37^{\circ} \mathrm{C}$. The pSuper vector was digested with $\mathrm{BgIII}$ and HindIII and then dephosphorylated with calf intestinal phosphatase for 1 hour at $37^{\circ} \mathrm{C}$. The ligation reaction was then set up over night at $16^{\circ} \mathrm{C}$ as shown in Table 2.4.2.

Table 2.4.2 PCR reaction for generation of shRNA

\begin{tabular}{ll} 
Component & Amount \\
\hline pSuper vector & $1 \mu \mathrm{l}$ \\
10x ligation buffer & $2 \mu \mathrm{l}$ \\
ATP & $0.5 \mu \mathrm{l}$ \\
Oligonucleotide mixture & $15,5 \mu \mathrm{l}$ \\
T4 ligase & $1 \mu \mathrm{l}$ \\
\hline Final volume $\left(\mathrm{ddH}_{2} \mathrm{O}\right)$ & $20 \mu \mathrm{l}$ \\
\hline
\end{tabular}




\subsubsection{Site-directed mutagenesis}

Primers for FBXO7 mutant generation were designed as suggested in the QuickChange Site-Directed Mutagenesis Kit (Agilant Technologies) (Papworth et al., 1996). Sequences are shown in Table 2.4.3.

Table 2.4.3 PCR reaction for generation of point mutations

\begin{tabular}{lll}
\hline Mutant & Sense primer & Antisense primer \\
\hline Fbxo7 T21M & cccgagacggagccgatgctggggcatttgcgc & gcgcaaatgccccagcatcggctccgtctcggg \\
Fbxo7 M115I & tccaatcagactagcatccaggatgaacaacca & tggttgttcatcctggatgctagtctgattgga \\
Fbxo7 R378G & aggttttatatctgggtgattttcgagacaat & attgtctcgaaaatcacccagatataaaaacct \\
Fbxo7 M498X & $\begin{array}{l}\text { cccatcttgccagggtgaggcggccccaatgac } \\
\text { ctgaaagctgagtctgtggagaacggcatggag } \\
\text { Pi31 V83E/I90E }\end{array}$ & $\begin{array}{l}\text { cagcacgttgatctccatgccgttctccacaga } \\
\text { ctcagcttcag }\end{array}$ \\
\hline
\end{tabular}

Reaction was set up as indicated in Table 2.4.4.

Table 2.4.4 PCR reaction for site-directed mutagenesis

\begin{tabular}{ll} 
Component & Amount \\
\hline $10 x$ Pfu buffer + MgSO4 & $5 \mu \mathrm{l}$ \\
$10 \mathrm{ng}$ dsDNA template & $1 \mu \mathrm{l}$ \\
$15 \mathrm{pmol}$ sense primer & $1.5 \mu \mathrm{l}$ \\
$15 \mathrm{pmol}$ asense primer & $1.5 \mu \mathrm{l}$ \\
$0.2 \mathrm{mM}$ dNTP mixture & $0.4 \mu \mathrm{l}$ \\
Pfu DNA polymerase & $1 \mu \mathrm{l}$ \\
\hline Final volume $\left(\mathrm{ddH}_{2} \mathrm{O}\right)$ & $50 \mu \mathrm{l}$ \\
\hline
\end{tabular}

Site-directed mutagenesis was then carried out in a PCR Thermocycler in the program shown in table 2.4.5.

Table 2.4.5 PCR program for site-directed mutagenesis

\begin{tabular}{llll} 
Step & Cycles & Temperature & Duration \\
\hline 1 & 1 & $95^{\circ} \mathrm{C}$ & 30 seconds \\
\hline 2 & 13 & $95^{\circ} \mathrm{C}$ & 30 seconds \\
& & $55^{\circ} \mathrm{C}$ & 1 minute \\
& & $68^{\circ} \mathrm{C}$ & 11.2 minutes \\
\hline
\end{tabular}


After reaction was terminated, $1 \mu \mathrm{l} \mathrm{Dpnl}$ was added to the mixture for 1 hour at $37^{\circ} \mathrm{C}$ to digest the methylated original strand. Bacteria were transformed with $1 \mu$ l reaction mixture, DNA extracted from colonies and sent for sequencing.

\subsubsection{Generation of FBXO7 FP-domain deletion construct}

Primers were designed for deletion of FP-domain of FBXO7 and amplification of the Nterminal and C-terminal fragments were then carried out simultaneously as described below (Table 2.4.6 and 7).

Table 2.4.6 PCR reaction for fragment amplification

\begin{tabular}{ll}
\hline Component & Amount \\
10x Pfu buffer + MgSO4 & $5 \mu \mathrm{l}$ \\
$100 \mathrm{ng}$ dsDNA template & $10 \mu \mathrm{l}$ \\
$10 \mathrm{pmol}$ sense primer (FBXO7 forward/N-terminal) & $1 \mu \mathrm{l}$ \\
$10 \mathrm{pmol}$ asense primer (C-terminal/FBXO7 reverse) & $1 \mu \mathrm{l}$ \\
$0.2 \mathrm{mM}$ dNTP mixture & $0.4 \mu \mathrm{l}$ \\
Pfu DNA polymerase (Fermentas) & $0.5 \mu \mathrm{l}$ \\
\hline Final volume $\left(\mathrm{ddH}_{2} \mathrm{O}\right)$ & $50 \mu \mathrm{l}$ \\
\hline
\end{tabular}

Table 2.4.7 PCR program for fragment amplification

\begin{tabular}{llll} 
Step & Cycles & Temperature & Duration \\
\hline 1 & 1 & $94^{\circ} \mathrm{C}$ & 30 seconds \\
\hline 2 & 13 & $94^{\circ} \mathrm{C}$ & 30 seconds \\
& & $53^{\circ} \mathrm{C}$ & 30 seconds \\
& & $72^{\circ} \mathrm{C}$ & 2 minutes/ kb plasmid \\
\hline
\end{tabular}

The two PCR-reaction mixtures were then run on a $1 \%$ agarose gel, cut out and purified using the NucleoSpin Plasmid kit. DNA was eluted in $30 \mu \mathrm{l} \mathrm{H}_{2} \mathrm{O}$. The fusion PCR reaction was then carried out as indicated below (Table 2.4.8) with the same Thermocycler settings as shown above. 
Table 2.4.8 PCR reaction for fragment fusion

\begin{tabular}{ll}
\hline Component & Amount \\
\hline 10x Pfu buffer + MgSO4 & $5 \mu \mathrm{l}$ \\
PCR C-terminal fragment & $1 \mu \mathrm{l}$ \\
PCR N-terminal fragment & $1 \mu \mathrm{l}$ \\
$10 \mathrm{pmol}$ sense primer & $1 \mu \mathrm{l}$ \\
$10 \mathrm{pmol}$ a-sense primer & $1 \mu \mathrm{l}$ \\
$0.2 \mathrm{mM}$ dNTP mixture & $0.4 \mu \mathrm{l}$ \\
Pfu DNA polymerase & $0.5 \mu \mathrm{l}$ \\
\hline Final volume $\left(\mathrm{ddH}_{2} \mathrm{O}\right)$ & $50 \mu \mathrm{l}$ \\
\hline
\end{tabular}

PCR reaction was again run on $1 \%$ agarose gel and cleaned up as described above followed by digestion with $\mathrm{Kpnl}$ and $\mathrm{EcoRI}$ at $37^{\circ} \mathrm{C}$ for 1 hour. Vector was digested by the same enzymes. The product was again cleaned up and DNA concentration was measured. The ligation reaction was carried out over night at $16^{\circ} \mathrm{C}$ as indicated below (Table 2.4.9).

Table 2.4.9 Ligation of fragment into vector

\begin{tabular}{ll}
\hline Component & Amount \\
\hline 10x T4 ligase buffer & $0.88 \mu \mathrm{l}$ \\
$0.04 \mu \mathrm{g}$ dsDNA template & $1 \mu \mathrm{l}$ \\
$0.5 \mu \mathrm{g}$ PCR product & $1 \mu \mathrm{l}$ \\
$10 \mathrm{mM}$ ATP & $0.7 \mu \mathrm{l}$ \\
T4 DNA ligase & $1 \mu \mathrm{l}$ \\
\hline Final volume $\left(\mathrm{ddH}_{2} \mathrm{O}\right)$ & $10 \mu \mathrm{l}$ \\
\hline
\end{tabular}

\subsubsection{Bacterial transformation and selection of positive clones}

PCR-reaction products or purified DNA were used to transform competent $\mathrm{DH} 5 \alpha$ cells. Bacteria were thawed on ice and reaction mixture added. This was incubated for 30 minutes prior to a 90 -second heat-shock at $37^{\circ} \mathrm{C}$. Liquid $2 \times Y T$ medium was added to the bacteria and incubated for 45 minutes at $37^{\circ} \mathrm{C}$. Bacteria were then transferred to $2 \times Y T$ agar plates with ampicillin/kanamycin and incubated over night at $37^{\circ} \mathrm{C}$. DNA was extracted from clones using the NucleoSpin Plasmid kit. Positive clones were selected after digestion with appropriate enzymes and sent for sequencing in the AGCT facility (MPI of Experimental Medicine, Göttingen). 


\subsection{Cell culture}

\subsubsection{HEK293T cells}

All cell work was performed under sterile conditions in a safety hood. HEK293T cells (Graham et al., 1977) were grown in DMEM supplemented with $10 \%$ FBS and $1 \%$ GlutaMAX ${ }^{\mathrm{TM}}$. Cells were cultured in an incubator at $37^{\circ} \mathrm{C}$ with $5 \% \mathrm{CO}_{2}$. When cells were confluent, they were washed in PBS prior to 2 minutes incubation with trypsin-EDTA at $37^{\circ} \mathrm{C}$. Fresh medium was added and cells were scraped off and centrifuged at $4^{\circ} \mathrm{C}$ at 800 rpm for $5 \mathrm{~min}$. The supernatant was discarded and cells resuspended in $5 \mathrm{ml}$ of new medium. Cells were then diluted 1:5 and plated in either a $10 \mathrm{~cm}$ dish or 6-well plates for maintenance or transfection respectively.

\subsubsection{Cerebellar granule neuron culture}

Cerebellar granule neurons were isolated and cultured as previously described (Bilimoria and Bonni, 2008). Cerebella of rat pups at P6 were dissected out and washed in HHGN. Tissue was digested with TDn for 20 minutes at $37^{\circ} \mathrm{C}$, then washed $3 \mathrm{x}$ in $\mathrm{HHGN}$ and triturated in DnB until the cell-suspension was homogenous. Cells were then spun down at $800 \mathrm{rpm}$ for 5 minutes and resuspended in CBC. Cells were counted in a haemocytometer using Trypan Blue and 20 million neurons plated on 10 centimeters dishes, coated in poly-ornithine. At DIV1, $10 \mathrm{mM}$ - - -mitotic agent cytosine- $\beta$-Darabinofuranoside (AraC) was added to the medium to abolish growth of proliferating cells such as astrocytes. At DIV3, $35 \mu \mathrm{l}$ of $1 \mathrm{M}$ glucose was added per $\mathrm{ml}$ of media to replenish the carbon source in the medium.

\subsubsection{Plasmid transfection of HEK293T cells}

Transfection of HEK293T cells was carried out as previously described (Konishi et al., 2004). 0.5-2 $\mu \mathrm{g}$ of plasmid and $50 \mathrm{ng}$ of GFP (C1-GFP, Invitrogen) was added to doubledestilled water to a volume of $90 \mu \mathrm{l}$. $10 \mu \mathrm{l}$ of $\mathrm{CaCl}_{2}$ was added to the mixture. Then $100 \mu \mathrm{l}$ 2xHBSS buffer was carefully bubbled into the plasmid solution. The solution was allowed to precipitate for 5 minutes at RT before being carefully added to the cell medium. The plate was gently shaken to mix the transfection solution with the medium and placed back into the incubator for 2-4 days before cell lysis. Transfection efficiency was monitored by visual inspection of GFP expression with an inverted fluorescent microscope (Nikon). 


\subsection{Biochemical experiments}

\subsubsection{Protein lysis and Bradford assay}

Tissue and cells were lysed in TX100 buffer and mechanically processed either with a cell scraper, a dounce or a homogenizer. The lysate was then left on ice for 30 minutes before centrifugation at $14000 \mathrm{rpm}$ for 5 minutes. Supernatant was transferred to a new Eppendorf tube and the pellet discarded.

Protein concentration was measured by the Bradford method (Bradford, 1976). Bovine serum albumine (BSA, Fermentas) of known concentration served as reference. 2-10 $\mu \mathrm{g}$ were diluted along with samples 1:500 in Bradford solution (1:5) in PBS. Absorbance was measured at wavelength of $595 \mathrm{~nm}$ in a Spectrophotometer (Amersham Biosciences). From the BSA measurements a standard curve was calculated and the sample protein concentration determined.

\subsubsection{SDS-PAGE and Western blotting}

Western blotting was performed according to standard protocols (Gallagher et al., 2008). Samples for Western blot analysis were boiled (except for transmembrane proteins) in SDS-sample buffer at $95^{\circ} \mathrm{C}$ for 5 minutes before being loaded into $10-12 \%$ denaturing Tris-SDS polyacrylamide gels (Table 2.6.1) and run by the SDS-PAGE electrophoresis system.

Table 2.6.1 Composition of Tris-SDS polyacrylamide gels

\begin{tabular}{|c|c|c|c|}
\hline Stacking gel & Amount & Separating gel & Amount \\
\hline Upper buffer & $25 \%$ & Lower buffer & $25 \%$ \\
\hline $\begin{array}{l}\text { Acrylamide/bis-acrylamide } \\
\text { (37.5:1, Th. Geyer) }\end{array}$ & $4 \%$ & $\begin{array}{l}\text { Acrylamide/bis-acrylamide } \\
(37.5: 1, \text { Th. Geyer })\end{array}$ & $10-12 \%$ \\
\hline $\begin{array}{l}\text { N',N',N',N'- Tetramethyethylenediamine } \\
\text { (TEMED) }\end{array}$ & $0.005 \%$ & TEMED & $0.005 \%$ \\
\hline Ammonium per sulphate (APS) & $0.05 \%$ & $\begin{array}{l}\text { Ammonium per sulphate } \\
\text { (APS) }\end{array}$ & $0.05 \%$ \\
\hline Final volume $\left(\mathrm{ddH}_{2} \mathrm{O}\right)$ & $5 \mathrm{ml}$ & Final volume $\left(\mathrm{ddH}_{2} \mathrm{O}\right)$ & $7.5 \mathrm{ml}$ \\
\hline
\end{tabular}

A pre-stained protein ladder (Fermentas) was always run next to the samples. Electrophoresis of protein samples was done at $35 \mathrm{~mA}$ per gel for 75 minutes in running buffer. Samples were then transferred onto nitrocellulose membranes (Protran BA85, VWR, Germany) in transfer buffer at $280 \mathrm{~mA}$ for 90 minutes. The membranes were next blocked using 4\% non-fat dried milk powder (Granovita GmbH, Germany) in PBST for 30 
minutes and then incubated over night or for 1 hour at RT with primary antibody in $3 \%$ BSA/PBST. Blots were washed 3x10 minutes in PBST and incubated with secondary antibodies (peroxidase conjugated, diluted 1:10 000 in 4\% milk/PBST) for $1 \mathrm{hr}$ at RT before another 3x 10-minute washes in PBST. Protein bands were detected on X-ray films (High performance Hyperfilms, Thermo Scientific, Pierce) by enhanced chemiluminescence, a substrate for peroxidase. Films were developed on a Kodak imaging station.

\subsubsection{Co-immunoprecipitation (Co-IP) analysis}

Co-IP analyses were modified from standard protocols (Bonifacino et al., 2001) and carried out on HEK293T cells transfected with plasmids to exogenously express or suppress proteins of interest. Cells were lysed in Co-IP buffer. Lysates were incubated with c-Myc or Flag antibodies ( $0.8 \mu \mathrm{g}$ of antibodies in $1 \mathrm{mg}$ lysate) at $4^{\circ} \mathrm{C}$ for 2.5 hours in a tumbler and subsequently with Protein A-Sepharose beads for 1 hour. The proteinbound beads were washed three times with Triton X-100 buffer and once with PBS. The bound protein was eluted by boiling the beads in SDS buffer.

\subsubsection{Subcellular fractionation}

Subcellular fractionation was performed as previously described (Konishi et al., 2004). Briefly, neurons in culture were scraped into detergent-free buffer $A$ and mechanically disrupted using a 2-ml dounce homogenizer. Nuclei were spun down at $500 \mathrm{~g}$ at $4{ }^{\circ} \mathrm{C}$ and the supernatant was collected as the cytoplasmic fraction. Nuclei were subjected to one wash in $0.1 \%$ NP40-supplemented buffer $A$ and then lysed in buffer $C$ and pelleted at maximum speed $(18,400 \mathrm{~g})$ at $4^{\circ} \mathrm{C}$. The supernatant was collected as the nuclear fraction. Fractions were subjected to SDS-PAGE and Western blot analysis as described.

\subsubsection{Purification of GST fusion proteins}

Previously generated (Sabitha Joseph) pGEX construct-transformed Escherichia coli (strain BL21) were grown to OD 0.5 in 2xYT medium, induced with $1 \mathrm{mM}$ isopropyl $\beta$-D-1thiogalactopyranoside (IPTG) for $3.5 \mathrm{~h}$ and sonicated in Co-IP buffer. The GST fusion proteins were purified from bacterial lysates by incubation with glutathione-coupled sepharose beads.

\subsubsection{GST pull-down of proteasomes}

The pull-down was modified from a previously published protocol (Besche and Goldberg, 2012). Whole brains were lysed in proteasomal lysis buffer and concentration adjusted to $2 \mathrm{mg} / \mathrm{ml}$. $10 \mu \mathrm{g} \mathrm{GST}$ protein and $40 \mu \mathrm{l}$ of $50 \%$ GST-beads was added to $1 \mathrm{ml}$ of lysate and 
incubated rotating at $4^{\circ} \mathrm{C}$ for 1 hour as a pre-clear step. Lysates were then spun down for $5 \mathrm{~min}$ at $14000 \mathrm{rpm}$ at $4^{\circ} \mathrm{C}$. $10 \mu \mathrm{g}$ GST or GST-Rad23 fusion protein was added to supernatant and incubated for 2.5 hours at $4^{\circ} \mathrm{C}$ before addition of $40 \mu \mathrm{l}$ of $50 \%$ GSTbeads and further incubation for another 1.5 hour at $4^{\circ} \mathrm{C}$. Beads were washed four times with ice-cold $0.1 \%$ NP40/PBS and boiled with SDS-sample buffer for 5 minutes at $95^{\circ} \mathrm{C}$. Samples were loaded into an SDS-PAGE gel and Western blotting followed.

\subsubsection{Proteasomal activity assay}

Proteasomal activity assays were carried out as previously described (Kisselev and Goldberg, 2005). Cells were lysed in proteasomal lysis buffer. $12 \mu \mathrm{g}$ of cell lysate was incubated with proteasomal activity buffer containing in addition $0.5 \mathrm{mg} / \mathrm{ml} \mathrm{BSA}$ and proteasomal chymotrypsin-like activity substrate, $100 \mu \mathrm{M}$ Suc-LLVY-amc. Fluorescence was measured using a fluorescent plate reader (Wallac 1420 VICTOR $^{\text {TM }}$ ) with 355/460 $\mathrm{nm}$ exitation/emission filters.

\subsubsection{Oxyblot analysis}

The OxyBlot ${ }^{\mathrm{TM}}$ Protein Oxidation Detection Kit (Millipore) was used to detect oxidized side chains of proteins in brain lysates as descibed in manufacturers protocol. Cortical tissue was lysed in Tx100 buffer with freshly added $3 \mu \mathrm{g} / \mathrm{ml}$ aprotinin, $1 \mu \mathrm{g} / \mathrm{ml}$ leupeptin, $50 \mathrm{mM}$ DTT and $1 \mu \mathrm{g} / \mathrm{ml}$ pepstatin. $5 \mu \mathrm{l} 12 \%$ SDS was added to $10 \mu \mathrm{g} / \mu \mathrm{l}$ protein in $5 \mu \mathrm{l}$ lysate. Two samples were prepared for each animal. $10 \mu \mathrm{LNPH}$ was added to one sample, while 10 $\mu \mathrm{l}$ derivatization control was added to the other and incubated for 15 minutes. $7.5 \mu \mathrm{l}$ neutralization solution was added and samples subjected to SDS-PAGE and Western blot analysis with the $\alpha$-DNP antibody.

\subsubsection{High Performance Liquid Chromatography (HPLC)}

Striata from mice, sacrificed by cervical dislocation, were dissected out on ice. HPLC was performed as previously described (Tonges et al., 2012). Tissue was homogenized in a Precellys $24 \AA$ bead mill homogenizer (Peqlab, Erlangen, Germany) in $0.1 \mathrm{M}$ perchloric acid, $50 \mu \mathrm{l} / \mathrm{mg}$ striatal tissue. Sample was subjected to centrifugation at $13.4 \mathrm{~g}$ for $5 \mathrm{~min}$, supernatant removed and centrifuged again at $13.4 \mathrm{~g}$ for $10 \mathrm{~min}$ at $4^{\circ} \mathrm{C} .20 \mu \mathrm{l}$ of supernatant was injected onto a C18 reverse-phase HR-80 catecholamine column (ESA, Bedford, MA, USA). HPLC with electrochemical detection was used to quantify dopamine, 3,4-dihydroxyphenylacetic acid (DOPAC) and homovanillic acid (HVA). Mobile phase had a flow rate of $0.4 \mathrm{ml} / \mathrm{min}$. Peaks were aquired by an ESA Coulochem III (5010) detector $(\mathrm{E} 1=50 \mathrm{mV}, \mathrm{E2}=400 \mathrm{mV})$. The Chromeleon computer system (Dionex, Idstein, 
Germany) was used to collect and process data. HPLC measurements were performed by Dr. Lars Tatenhorst.

\subsection{FBXO7 transgenic mouse line}

Ethics Statement: All experiments involving live animals have been conducted according to the animal protocol approved by the "Verbraucherschutz und Lebensmittelsicherheit" of Lower Saxony, Germany (33.11.42502-04-11/0632).

\subsubsection{Generation of FBXO7 transgenic mouse line}

FBXO7 deficient mice were created by homologous recombination in mouse JM8.N4 ES cells (23037 EUCOMM). FBXO7 genomic sequence containing exon 4 was exchanged with a neo:lacZ cassette driven by the L1L2_Bact_P promotor. Chimeras were generated by injection of targeted ES cells into C57BL/6N blastocysts. The line was kept in a pure C57BL/6N background.

\subsubsection{Isolation of genomic DNA and genotyping}

Isolation of mouse DNA followed a simplified standard protocol (Gross-Bellard et al., 1973). Tail tissue from animals was lysed in genotyping lysis buffer for minimum 2 hours at $55^{\circ} \mathrm{C}$. Samples were resuspended and spun down for 10 minutes at $14000 \mathrm{rpm}$. DNA was extracted by addition of $98 \%$ ethanol followed by centrifugation for 10 minutes at 14 $000 \mathrm{rpm}$. DNA was washed once with 70\% ethanol and resuspended in 50-100 $\mu \mathrm{l} \mathrm{H}_{2} \mathrm{O}$. PCR-reaction was carried out according to the GoTaq ${ }^{\circledR}$ DNA polymerase system as described below (Table 2.7.1-3). After completion samples were run on a $1 \%$ agarose gel at $50 \mathrm{~V}$ with $\mathrm{GeIRed}^{\mathrm{TM}}(1: 100000)$ added for visualization of sample with a UV-detector.

Table 2.7.1 Genotyping PCR reaction

\begin{tabular}{ll} 
Component & Amount \\
\hline $5 x$ GoTaq buffer & $5 \mu \mathrm{l}$ \\
Genomic DNA & $0.5 \mu \mathrm{l}$ \\
10 pmol sense primer & $1 \mu \mathrm{l}$ \\
10 pmol antisense primer & $1 \mu \mathrm{l}$ \\
$2.5 \mathrm{mM}$ dNTP mixture & $0.4 \mu \mathrm{l}$ \\
GoTaq DNA polymerase & $0.1 \mu \mathrm{l}$ \\
\hline Final volume $\left(\mathrm{ddH}_{2} \mathrm{O}\right)$ & $25 \mu \mathrm{l}$ \\
\hline
\end{tabular}


Table 2.7.2 Genotyping PCR program

\begin{tabular}{llll} 
Step & Cycles & Temperature & Duration \\
\hline 1 & 1 & $95^{\circ} \mathrm{C}$ & 3 minutes \\
\hline 2 & 28 & $95^{\circ} \mathrm{C}$ & 30 seconds \\
& & $51^{\circ} \mathrm{C}$ & 30 seconds \\
& $72^{\circ} \mathrm{C}$ & 2 minutes/ kb DNA-fragment \\
\hline
\end{tabular}

Table 2.7.3 Primers used for genotyping

\begin{tabular}{llll} 
Gene & Sense primer & Antisense primer & Size \\
LacZ casette & attccagctgagcgccggtcgc & gcgagctcagaccataacttcgtata & $400 \mathrm{bp}$ \\
Fbxo7 loxP2 & tcagcatgggtttgttaagcatctacta & ggtctagatatctcgacataacttcgtata & $600 \mathrm{bp}$ \\
Fbxo7 WT & gggctgtatgaaggaagtgctatt & ccctgagagtgaagggtgctgttc & $800 \mathrm{bp}$ \\
Cre & cagggtgttataagcaatccc & cctggaaaatgcttctgtccg & $250 \mathrm{bp}$ \\
\hline
\end{tabular}

\subsubsection{RNA isolation, cDNA synthesis and quantitative PCR}

For assessment of mRNA expression level, total RNA from tissue was isolated using the TRIZOL reagent (Invitrogen) (Chomczynski, 1993). Tissue was homogenized with TRIZOL on ice using a Dounce. Chloroform was added 1:5 and samples vortexed before 15 minutes incubation on ice. Samples were then centrifuged for 15 minutes at $4^{\circ} \mathrm{C}, 14$ $000 \mathrm{rpm}$, and supernatant transferred to new tube. Equal volume of isopropanol was added followed by 15 minutes incubation on ice and 15 minutes centrifugation at $4^{\circ} \mathrm{C} 14$ $000 \mathrm{rpm}$. Samples were washed once with $70 \%$ ethanol and resuspended in $50 \mu \mathrm{l} \mathrm{H}_{2} \mathrm{O}$. RNA concentration was measured in a spectrophotometer. cDNA was then synthesized using the SuperScript III First-Strand Synthesis System (Invitrogen) (Table 2.7.4-5):

Table 2.7.4 Reaction for cDNA synthesis 1

\begin{tabular}{ll} 
Component & Amount \\
RNA & $3 \mu \mathrm{l}$ \\
$50 \mu \mathrm{M}$ Oligo dT & $1 \mu \mathrm{l}$ \\
$10 \mathrm{mM}$ dNTP & $1 \mu \mathrm{l}$ \\
\hline Final volume $\left(\mathrm{ddH}_{2} \mathrm{O}\right)$ & $10 \mu \mathrm{l}$ \\
\hline
\end{tabular}

The reaction was incubated for 5 minutes at $65^{\circ} \mathrm{C}$ followed by 1 minute on ice. Reverse transcriptase mixture was prepared as indicated below and added to each sample. 
Table 2.7.5 Reaction for cDNA synthesis 2

\begin{tabular}{ll} 
Component & Amount \\
\hline $10 x$ reverse transcriptase buffer & $2 \mu \mathrm{l}$ \\
$25 \mathrm{mM} \mathrm{MgCl}_{2}$ & $4 \mu \mathrm{l}$ \\
$0.1 \mathrm{M} \mathrm{DTT}$ & $2 \mu \mathrm{l}$ \\
RNAse OUT & $1 \mu \mathrm{l}$ \\
SuperScript Reverse Transciptase & $1 \mu \mathrm{l}$ \\
\hline Final volume & $10 \mu \mathrm{l}$ \\
\hline
\end{tabular}

The reaction was then incubated 50 minutes at $50^{\circ} \mathrm{C}$ followed by 5 minutes at $85^{\circ} \mathrm{C}$ to stop reaction. Samples were chilled on ice and $1 \mu \mathrm{l}$ RNAse $\mathrm{H}$ was added to each and incubated at $37^{\circ} \mathrm{C}$ for 20 minutes. To amplify cDNA fragments, cDNA was mixed with the Power SYBR Green PCR Master Mix (Invitrogen) as indicated below (Table 2.7.6).

Table 2.7.6 Reaction for quantitative PCR

\begin{tabular}{ll} 
Component & Amount \\
\hline SYBR Green & $5 \mu \mathrm{l}$ \\
1 pmol sense primer & $0.1 \mu \mathrm{l}$ \\
1 pmol antisense primer & $0.1 \mu \mathrm{l}$ \\
cDNA & $0.14 \mu \mathrm{l}$ \\
\hline Final volume $\left(\mathrm{ddH}_{2} \mathrm{O}\right)$ & $10 \mu \mathrm{l}$ \\
\hline
\end{tabular}

Primers for quantitative PCR were designed to amplify a sequence of $150-180$ bp spanning an exon-intron border. Primers used in this study are listed in Table 2.7.7.

Table 2.7.7 Primers for quantitative PCR used in this study

\begin{tabular}{lll} 
Gene & Sense primer & Antisense primer \\
\hline FBXO7 & tggaagtcaagtggtgtatac & tactccagcagcaacgtagga \\
$\mathrm{PI} 31$ & cggtatgagtctaaggatgga & gtggaagtcactcaggtcttc \\
$\beta$-actin & atgccacaggattccatacc & cttcctccctggagaagagc \\
\hline
\end{tabular}




\subsection{Immunohistochemistry (IHC)}

\subsubsection{Transcardial perfusion and cryo-sectioning}

For histochemistry, mice were anesthetized with $100 \mathrm{mg} / \mathrm{kg}$ ketamin and $10 \mathrm{mg} / \mathrm{kg}$ xylazin in PBS, $100 \mu \mathrm{l}$ were injected per 10 grams of weight. After deep tendon reflexes were abolished, mice were perfused with $10 \mathrm{ml}$ of PBS followed by $10 \mathrm{ml} 4 \%$ PFA in PBS at a rate of $12 \mathrm{rpm}$ with an infusion pump. Brains were post-fixed overnight at $4^{\circ} \mathrm{C}$ in $4 \%$ PFA in PBS and then kept in $30 \%$ sucrose in PBS for 24 hours at $4^{\circ} \mathrm{C}$. They were then embedded in equal volumes of OCT and $30 \%$ sucrose in PBS and stored at $-80^{\circ} \mathrm{C}$. Coronal sections of $30 \mu \mathrm{m}$ were cut on a cryostat and kept free floating in PBS with $0.02 \%$ $\mathrm{NaNH}_{3}$ at $4^{\circ} \mathrm{C}$.

\subsubsection{Fluorescent IHC}

A modified protocol for staining of free-floating sections was followed (Hirrlinger et al., 2006). Sections were washed two times with PBST (1xPBS, 0.2\% Tween 20) and permeabilized for 30 minutes with $0.5 \%$ Triton-X 100 in PBS followed by blocking in blocking solution (10\% goat serum, 2\% BSA, 0.5\% TX100 in PBS) for an hour at RT. Primary antibody was appropriately diluted in blocking solution without TX100 and the sections were incubated on a shaker over night at $4^{\circ} \mathrm{C}$. Sections were then washed three times for 10 minutes in PBST before incubation in secondary antibody in blocking solution without TX100 for 1 hour at RT. To stain nuclei, DAPI was diluted 1:8000 in $\mathrm{H}_{2} \mathrm{O}$ and applied to sections for 5 minutes at RT. At last, sections were washed three times 5 minutes in PBST for and mounted with Mowiol mounting medium on poly-lysine coated glass slides (Macharey-Nagel).

\subsubsection{DAB staining}

DAB and Nissl staining protocols were modified from Tonges et al. (Tonges et al., 2012). Sections were washed three times 5 minutes in TBS on a shaker at RT before permeabilization and peroxidase suppression in $40 \%$ methanol/ $1 \% \mathrm{H}_{2} \mathrm{O}_{2}$ in TBS for 15 minutes at RT. Sections were washed again in the same manner and then blocked in $5 \%$ goat serum in TBS for 1 hour at RT. Primary antibody was applied in the appropriate dilution in $2 \%$ goat serum in TBS for 48 hours at $4^{\circ} \mathrm{C}$ shaking. Sections were then washed as before and secondary antibody was diluted in TBS and applied to sections for 2 hours at RT on shaker and then washed as before. 1 drop solution $A$ and 1 drop solution $B$ were mixed in TBS (PK-4000 Peroxidase Standard kit) and allowed to incubate for 45 minutes before application to the sections for 2 hours at RT and then washed as before. 2 drops of 
buffer, 2 drops of DAB, 2 drops of $\mathrm{H}_{2} \mathrm{O}_{2}$ and 2 drops of nickel solution were mixed in $5 \mathrm{ml}$ $\mathrm{H}_{2} \mathrm{O}$ (SK-4100 kit) and applied to sections for 10 minutes at RT. Reaction was stopped with $\mathrm{H}_{2} \mathrm{O}$ for 5 minutes and sections mounted on poly-lysine coated slides (MachareyNagel) and allowed to dry for 5 days.

\subsubsection{Nissl staining}

Dried DAB stained sections were first hydrated through an alcohol row (3x Xylol, 100\% isopropanol, $100 \%$ ethanol, $90 \%$ ethanol, $70 \%$ ethanol, $\mathrm{H}_{2} \mathrm{O}$ ) each for 5 minutes before staining with Nissl staining solution for 7 minutes. Sections were then dehydrated through the inverse alcohol row and mounted with Eukitt mounting medium.

\subsection{Behavioral analyses}

All mice were housed in the animal facility of the MPI of Experimental Medicine in Göttingen, Germany. Mice were group-housed in transparent polycarbon cages (Makrolon type II cage, 22x16x14 cm) with access to food and water ad libitum. For analyses of the FBXO7 conventional knock-out mice, mixed gender mice of age P18 were used in all experiments. Mice were allowed to acclimatize in testing facility for $30 \mathrm{~min}$ before test. Due to the extreme difference in weight between FBXO7-/- and their littermates, experimenter could not be kept blind to genotype. For animals from conditional FBXO7floxed lines and conventional $F B X O 7+/$ - versus wild type mice older than $\mathrm{P} 18$, male mice were used. All males were allowed to acclimatize in the behavioral facility one week before testing occurred and spent at least 30 minutes in test room before every test. Experiments were conducted in an order ranking from least to most invasive: elevated plus maze for anxiety, open field for exploratory behaviour and ambulation, pole test, Inverted grid, wire hang, rotarod and balance beam for motor endurance and coordination, Y-maze for memory, marble burying for stereotypies and buried food for olfaction assessment. Between each mouse the equipment was washed first with water, then $70 \%$ ethanol, followed by a last wash with water to clear any odor traces.

\subsubsection{Surface righting reflex}

This protocol was modified from Dr. Martesa Tantra's protocol (Dere et al., 2014). Pups of age P5 were placed on their backs on a flat surface covered with a tissue paper underneath a warming lamp. Time spent to right themselves were recorded in three consecutive trials with an intra test interval of 5 minutes. Cut-off time was set to 30 seconds and average time was calculated. 


\subsubsection{Tail suspension test}

The tail suspension test protocol was modified from a scoring protocol for cerebellar ataxia (Guyenet et al., 2010). Mice were suspended by the base of their tail for three consecutive 10 seconds trial with a resting phase of 1 minute in between. Mice were scored as normal ( 0 ) if they extended both hind limbs for more than $50 \%$ of the time. They were scored as 1 if they retracted one hind limb for more than $50 \%$ of the time and 2 if they partially retracted both hind limbs for more than $50 \%$ of the time. The worst manifestation of the phenotype, fully retracted hind limbs towards the belly, was scored as 3. An average score was given to each mouse.

\subsubsection{Kyphosis}

The kyphosis assessment was performed as instructed in the cerebellar ataxia scoring protocol (Guyenet et al., 2010). Mice were observed walking in an open field and scored as normal (0) when they showed no sign of arched back. They were given a score of 1 if the spine was arched while sitting, but could be fully extended during movement and a score of 2 if the sine curvature persisted mildly while walking. A score of 3 was given to severe curvature of the spine that persisted while walking or sitting.

\subsubsection{Gait analysis}

Gait analysis was conducted as previously described (Guyenet et al., 2010). The mice were placed in an open field and observed while walking. The criteria observed were: Is body weight supported equally on all limbs, does the abdomen touch the ground, do we observe tremor, limping or abnormally placed feet during movement. Mice were scored from 0-3 based on the severity of the symptoms.

\subsubsection{Wire hang}

Wire hang is a test to measure muscle strength in mice. This test was modified from a previously described protocol (van Putten et al., 2010). The testing apparatus consisted of an $80 \mathrm{~cm}$ long; $1 \mathrm{~mm}$ wide steel wire suspended $30 \mathrm{~cm}$ above surface covered with soft tissue. Mice were placed with front paws holding the middle of the wire and hang time measured. Climbing to end of the wire was counted as maximal time (30 seconds). Each mouse was tried three times with an intra-test interval of 5 minutes and average hang time calculated. 


\subsubsection{Inverted grid}

Another test to measure muscle strength in mice is the inverted grid test (Tillerson and Miller, 2003). Here the mice were placed on top of the home cage lid and the lid was then inverted around $20 \mathrm{~cm}$ above cage. The time the mice could hold on to cage lid was measured three times with an intra test interval of 1 minute. Cut-off time was set at 30 seconds.

\subsubsection{Ledge test}

Ledge test was performed as described previously (Guyenet et al., 2010). Briefly, the mice were placed on the edge of the home cage and encouraged to cross over to the opposite side. Test was conducted three times with an inter-trial interval time of 5 min. Mice were scored from having a normal balance $(0)$ to unable to walk on ledge (3). A score of 1 was given for minor adjustment problems, while a score of 2 was given to extensive coordination problems or near-falls. Average score was calculated for each animal.

\subsubsection{Balance beam test}

Balance beam is a test to evaluate coordination and motor control in mice. The protocol was adapted from Luong et. al (Luong et al., 2011). Mice were placed on a beam of 12 $\mathrm{mm}$ width, $80 \mathrm{~cm}$ length, $50 \mathrm{~cm}$ above ground with home cage at the other end and encouraged to cross 3 times with 15 seconds rest in home cage between trials. A cloth, which served as a safety net, was elevated from ground underneath beam to prevent mice from being hurt by falling. After a 10 minutes rest, the procedure was repeated on a beam of $6 \mathrm{~mm}$ width. The training was repeated on two consecutive days before the test day where the time of three trials on each beam was recorded and animals scored according to number of slips on beam. A score of 7 was given to animals crossing with less then two slips, 6 was given to animals that slipped more than two times, but otherwise crossed fine. A score of 5 was given when one limb was partially dragged across beam, 4 when one limb was constantly dragged, or both limbs were partially dragged. A score of 3 means both hind limbs were dragged across while 2 was given to mice barely able to cross. When the mouse was not able to cross, a score of 1 was given.

\subsubsection{Pole test}

The pole test has previously been described as a method to assess dopaminergic neuron integrity (Ogawa et al., 1985). Animals were placed on a $50 \mathrm{~cm}$ (30 cm for P18 old animals) tall pole situated in the home cage, $1 \mathrm{~cm}$ in diameter, covered in gauze with 
head facing up and encouraged to climb down. The protocol was applied 5 times, with 30 seconds break in between, for each mouse on three consecutive days (for P18 only one day). On the last day time to turn around, face down, as well as total time spent descending pole was recorded manually by experimenter. The best try was counted.

\subsubsection{Rotarod}

Rotarod is a motoric test that measures motor function, comprising balance, endurance and coordination (Crawley, 2003). All experiments were conducted on a rotarod (Ugo Basile, Srl, Comerio, Italy) on an accelerating protocol of 4-40 rpm for 5 minutes. P18 conventional FBXO7-/- mice and controls were tested three times on 1 day with an intertrial interval of 20 min and average latency to fall was measured. Conditional Nex-Cre and $\mathrm{TH}-\mathrm{Cre}$; FBXO7fl/fl and conventional $\mathrm{FBXO7+/-} \mathrm{mice} \mathrm{with} \mathrm{controls} \mathrm{were} \mathrm{tested} \mathrm{with} \mathrm{the}$ same protocol on three consecutive days to also assess motor learning.

\subsubsection{Open field}

Open field analysis for P18 FBXO7-/- mice and controls was conducted as described previously (Biondi et al., 2010). Mice were placed in the center of a plastic container measuring $28 \times 28 \times 20 \mathrm{~cm}$ and observed for 5 minutes. The container was divided into 16 squares where the four in the middle were referred to as central and the remaining as peripheral squares. Number of peripheral and central crossings was recorded manually.

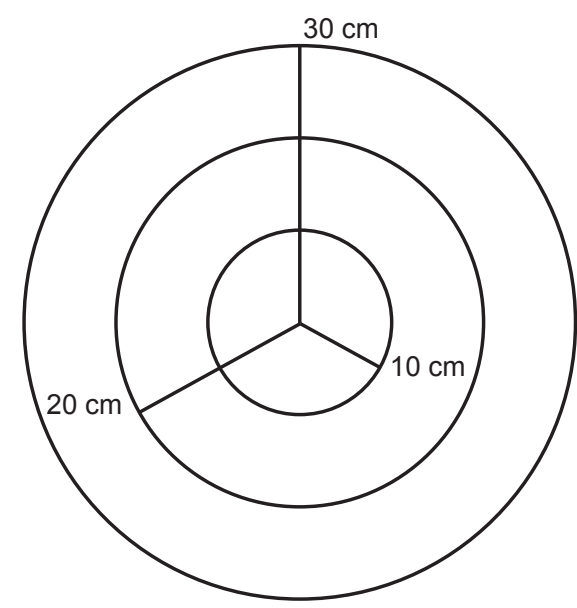

For older mice, a round open field of $60 \mathrm{~cm}$ diameter with $20 \mathrm{~cm}$ high walls was used. The field was divided into three zones as shown in Figure 2.1 (Dere et al., 2014). The animals were placed in the middle of the field and allowed to move freely for 7 minutes. The light intensity was set at 120 lux. The animal's movement was recorded with the Viewer animal tracking system

Figure 2.1 Schematic of open field for adult animals

\subsubsection{Elevated plus maze}

To test for anxiety-like behavior the elevated plus maze was used as previously described (Dere et al., 2014). Mice were placed facing the closed arm of an apparatus with two closed and two open arms. All arms measuring $5 \times 30 \mathrm{~cm}$ with the closed ones having walls of $15 \mathrm{~cm}$. The maze was elevated $40 \mathrm{~cm}$ above ground level with soft tissues placed 
under the open arms and the light intensity was set to 120 lux. Mice were allowed to freely explore the maze for 5 minutes, which was recorded by the Viewer software.

\subsubsection{Light avoidance test}

Another test for anxiety-like behavior is the light avoidance test (Belzung et al., 1987). Mice were placed in a cage with a light area, $25 \times 27 \times 30 \mathrm{~cm}$ and a dark area, $18 \times 27 \times 30$ $\mathrm{cm}$. The areas were connected through an opening, $7 \times 5 \mathrm{~cm}$. Animals were placed facing away from the opening in the light area, light intensity of 400 lux, and then spent 10 minutes freely exploring. The mouse was recorded using the Viewer animal tracking software. Movement within light area, crossings into dark area as well as latency to enter dark area and time spent in the different compartments was measured.

\subsubsection{Marble burying}

To assess the stereotypic burying the marble test can be used (Dere et al., 2014). 24 glass marbles were placed in a regular rectangular pattern of $4 \times 6$ on top of bedding in a cage measuring $55 \times 35 \times 20 \mathrm{~cm}$. Animals were allowed to explore cage freely for $30 \mathrm{~min}$ in light intensity of 10 lux. After that time, marbles were classified as buried when $3 / 4$ or more of marble were covered in bedding. Buried and moved marbles were counted and pattern assessed as organized or disorganized.

\subsubsection{Y-maze}

To address the issue of spatial memory a Y-maze test was employed (Swonger and Rech, 1972). Animals were placed in the middle of a Y-shaped maze with three arms measuring $7.5 \times 30 \times 15 \mathrm{~cm}$ spaced $120^{\circ}$ apart. Mice were allowed to explore freely for 7 min and the Viewer software recorded their movements. Sequence of arm entering was noted and ratio of triplicates ( $A B C, C B A, B C A, C A B, A C B, B A C)$ as well as total arm entries were counted.

\subsubsection{Olfaction test}

Olfaction was measured by introducing the mice to chocolate chip cereals (Dere et al., 2014). These were given into the home cage on the night before training, two cereals per mouse. The next day, the mice were placed into cages for measuring and covered in bedding at 10 lux. A chocolate chip cereal was placed on top of the bedding in either of four locations as shown in (Figure 2.2). The mice were allowed to explore the cage freely for 10 minutes. The procedure was repeated every 50 minutes for four times until all locations had been used. This protocol was applied on two consecutive days. After the second day the food was removed from the home cage and the animals left with only 
water over night. On the day of testing the chocolate chip cereal was buried in location $B$ and the mice placed in the middle of the cage and allowed to explore for maximal 5

\begin{tabular}{ll|l}
\hline A & B & $\begin{array}{l}\text { minutes. This was repeated after } 90 \text { minutes, then with the chip in } \\
\text { location C. After another } 90 \text { minutes a control trial was conducted, then } \\
\text { with the chip in location E, on top of the bedding. Each trial was recorded } \\
\text { by the Viewer software and time until retrieval of chip was measured. }\end{array}$ \\
& C & Figure 2.2 Schematic of olfaction test
\end{tabular}

\subsubsection{Sucrose preference test}

To assess whether the mice were affected by anhedonia, they were checked for sucrose preference (Strekalova and Steinbusch, 2010). Mice were single housed and given access to two bottles of drinking water, one of which contained $2 \%$ sucrose. The location of the bottles was switched every day to avoid location bias. The first three days were counted as acclimatization phase. From the third day on the bottles were weighed every day for four days and the ratio of amount of sucrose water consumed versus total consumption was measured.

\subsubsection{Laboratory animal behavior, observation, registration and analysis system (LABORAS)}

The LABORAS home cage behavioral monitoring system (Metris b.v., Hoofddorp, The Netherlands) is equipped with a sensor platform set on two force transducers and attached to an underlying plate (Dere et al., 2014). Home cages were placed onto the sensor platform, with the grid suspended above. Vibrations caused by animal movement were transformed into electrical signals and digitized. Data was processed, the signals assesed to specific behavioral categories and the dominating behavior at the time was measured by the LABORAS software. Amount of seconds spent performing a behavior and its frequency were calculated. Animals were allowed to acclimatize to the system for 1 hour, and recordings performed for 15 hours during the dark cycle. Experiment was performed by Anja Ronnenberg and data analyzed by Dr. Ekrem Dere.

\subsubsection{DigiGait analysis}

Gait parameters in mice were analyzed with the DigiGait ${ }^{\mathrm{TM}}$ imaging system (Mouse Specifics, Inc., Boston) (Hampton et al., 2004; Kale et al., 2004). Animals were placed on a transparent treadmill belt with adjustable speed. The walking mouse is recorded from below and movies acquired with the DigiGait ${ }^{\mathrm{TM}}$ Imager software (version 12.5) Animals were acclimated to the testing room for at least one week before the test. Prior to 
recordings, the animals were allowed to freely explore the treadmill compartment for 5 $\mathrm{min}$. The speed was slowly increased up to $25 \mathrm{~cm} / \mathrm{s}$ and running sessions, separated by a few minutes of rest in the cage, were recorded. The acquired movies were processed using the DigiGait ${ }^{\mathrm{TM}}$ Analysis software (version 14.0) and basic gait metrics calculated. Values for fore- and hindlimbs were calculated separately with values for right and left limb pooled. Experiment was performed by Camille Lancelin.

\subsection{Data analyses}

\subsubsection{Western blot quantification}

Developed films from Western blot analysis were scanned and subjected to densitometric analyses using the ImageJ Gel Analyzer plug-in. The intensity of each sample and the respective loading controls were measured and intensity relative to loading control was calculated. The first wild type sample was set as reference and values normalized to this.

\subsubsection{Stereological counting}

Stereological methodology for quantification of tyrosine hydroxylase-, or Nissl-positive cells in the substantia nigra has been previously described (Tonges et al., 2012). The Stereo Investigator software (Stereo Investigator 9.0, MicroBrightField Inc.) and a Zeiss Axioplan microscope were used to analyze every fourth histological section of the midbrain. A point grid was laid over the outlined Substantia nigra pars compacta. TH or Nissl-positive cells in one substantia nigra per animal were counted by the optical fractionator method ( $\times 40$ objective, counting frame $50 \times 50 \mu \mathrm{m}$ ). Nissl cells were counted to rule out down-regulation of the tyrosine hydroxylase enzyme as opposed to loss of neurons. Experiment was performed in a blinded manner.

\subsubsection{Quantification of IHC images}

Fluorescent images obtained by the Zeiss Axio Observer inverted microscope, were converted to 8-bit in ImageJ and a Bernsen local area threshold of 15 was applied. Ventral tegmental area and substantia nigra was delineated based on TH-positive area. The area fraction stained was measured.

\subsubsection{Statistical analysis}

All statistical analyses were performed with the GraphPad Prism 6.0 software unless otherwise stated. 
"There is nothing like looking, if you want to find something. You certainly usually find something, if you look, but it is not always quite the something you were after."

J.R.R. Tolkien, "The Hobbit"

\section{Results}

\subsection{FBX07 is ubiquitously expressed in rodents and present in the cytoplasm of cerebellar granule neurons}

FBXO7 was first identified in two large screens for F-box proteins (Cenciarelli et al., 1999; Winston et al., 1999). Winston and colleagues here looked at expression pattern of FBXO7 mRNA in various murine tissues and found FBXO7 mRNA to be expressed in brain, heart, kidney, liver, lung, skeletal muscle, pancreas and placenta, but absent from spleen, testis and embryonic tissue. A subsequent study found FBXO7 mRNA to be ubiquitously expressed in human tissue (Ilyin et al., 2000), which was later confirmed by histological analysis in human brain (Zhao et al., 2011). At the cellular level, FBXO7 distribution is debated. It was first shown that human FBXO7 was localized mainly to cytoplasm in cycling cells (Chang et al., 2006; Kirk et al., 2008; Laman et al., 2005), but later suggested that it might be present in the nucleus as well and actively exported out through Crm1 during cell cycle (Nelson and Laman, 2011). This was again challenged by two groups, who found that FBXO7 was localized mainly to the nucleus in HEK293T and SH-SY5Y cells as well as primary mouse hippocampal neurons (Zhao et al., 2011; Zhou et al., 2015). In this study, I use rodents to investigate the expression pattern of FBXO7 and to model PARK15 signs and symptoms.

\subsubsection{Expression pattern of FBXO7}

I first established the expression profile of FBXO7 by means of western blot analysis of rat tissue. I found the FBXO7 protein to be expressed in all tissues tested (Figure 3.1.1), contradicting the mRNA to a certain degree, as I also observed expression in the spleen (Winston et al., 1999). Here however, the antibody showed several bands of various size meaning it could be an unspecific interaciton.

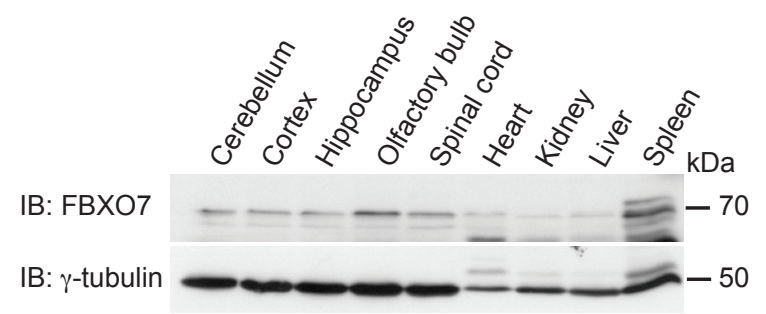

Figure 3.1.1 FBXO7 shows ubiquitous expression in rat tissue. Tissue samples from adult male rat, postnatal day $(P) 30$ were subjected to immunoblotting with the $\mathrm{FBXO7}$ antibody. $\gamma$-tubulin was used as loading control. 
I then analyzed the temporal expression pattern of FBXO7 in the brain. I immunoblotted tissue from rat cerebellum, hippocampus and cortex and found that FBXO7 is expressed in these brain regions throughout development. For cortex and hippocampus, I observed an age-dependent increase in FBXO7 expression, while I found an age-dependent decrease in the cerebellum (Figure 3.1.2).

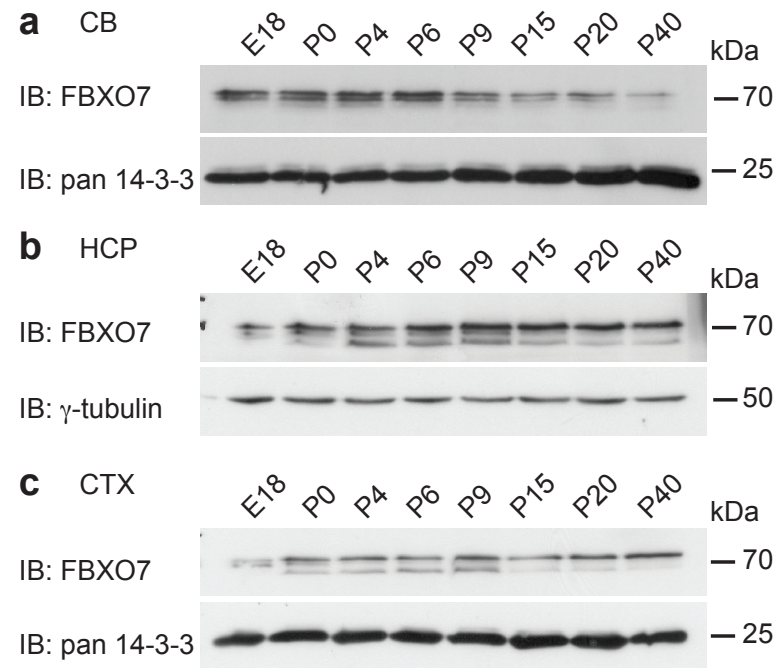

Figure 3.1.2 FBXO7 is expressed in the brain throughout development. Lysates from rat cerebellum (a), hippocampus (b) and cortex (c) at different developmental stages (embryonic day (E) 18 to P40) were subjected to immunoblotting with an FBXO7 antibody. Pan 14-3-3 or $\gamma$-tubulin was used as loading control. $\mathrm{CB}=$ cerebellum, $\mathrm{HCP}=$ hippocampus, $\mathrm{CTX}=$ cortex.

\subsubsection{FBXO7 is localized mainly in the cytoplasm in rat cerebellar granule neurons}

The localization of proteins within a cell can offer insight into their mode of function. I therefore carried out subcellular fractionation on cultured rat neurons from cerebellum (Figure 3.1.3), and found by subsequent immunoblotting that FBXO7 localized predominantly to the cytoplasm of these cells.

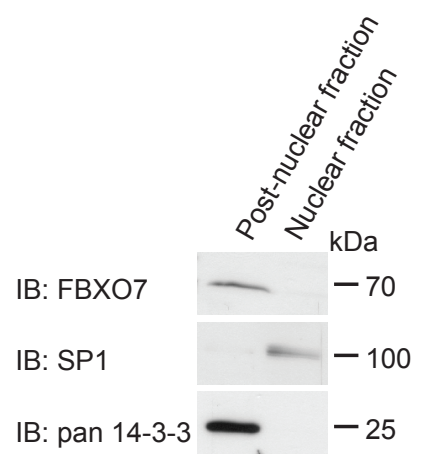

Figure 3.1.3 FBXO7 is localized mainly in the cytoplasm of cultured cerebellar granule neurons. Cultured cerebellar granule neurons, day in vitro (DIV) 9, from rat were subjected to subcellular fractionation followed by immunoblotting with an FBXO7 antibody. Pan 14-3-3 served as cytoplasmic and SP1 as nuclear control.

Taken together, I conclude that FBXO7 is present in various organs in rodents including the brain. It is expressed in cerebellum, hippocampus and cortex throughout development. At the cellular level, FBXO7 is mainly found in the cytoplasm of cultured rat granule neurons. 


\subsection{FBXO7 positively regulates proteasome activity}

Proteasomal dysfunction is one of the mechanisms suggested to contribute to the etiology of PD and inhibition of proteasomal activity has been shown to induce bradykinesia in rats (McNaught et al., 2002b). In our lab David Brockelt identified the proteasomal subunit, PSMA2, to be a direct interaction partner of FBXO7. To unravel the mechanistic pathway underlying the PARK15 syndrome, I therefore chose to focus on how FBXO7 impacts the ubiquitin proteasome system.

One of the known interaction partners of $\mathrm{FBXO} 7$ is $\mathrm{PI} 31$, a proteasomal regulator, which binds directly to FBXO7 via a shared FP domain (Kirk et al., 2008) (Figure 1.6). I created an FBXO7 mutant that lacked the FP domain and a PI31 mutant that had crucial uncharged amino acids converted into positively charged ones (V83E/I90E). Thereby I confirmed the interaction of the wild type proteins and the abolished interaction of the mutants through forward and reverse co-immunoprecipitation analysis (Figure 3.2.1a,b).

a

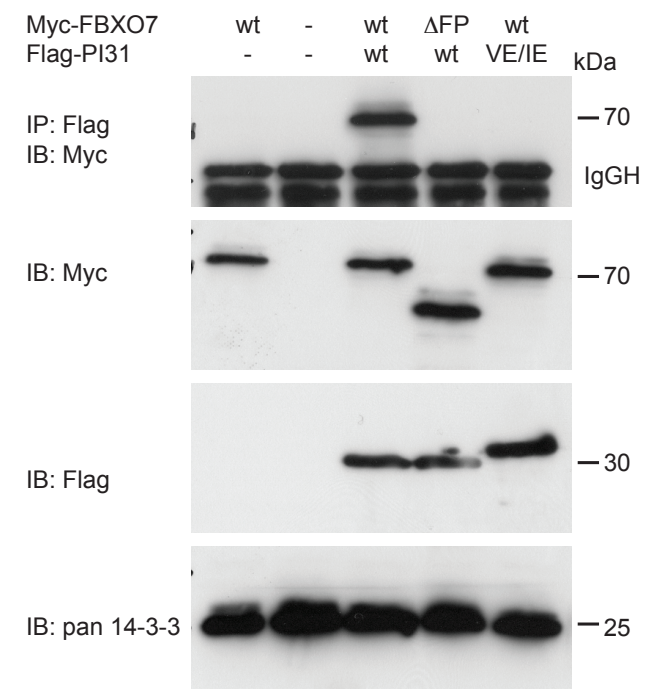

b

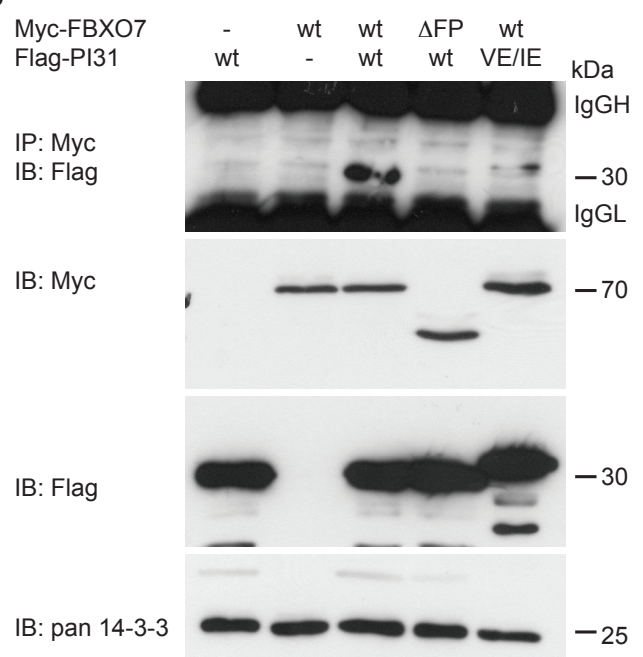

Figure 3.2.1 The FP domain of FBXO7 is required for interaction with PI31. Myc-tagged FBXO7 wild type (wt) or $\triangle \mathrm{FP}$ domain, was co-expressed with Flag-tagged PI31 wt or the V83E/I90E mutant, and control plasmids in HEK293T cells. Cells were lysed and lysate subjected to immunoprecipitation with an anti-Flag (a) or anti-Myc (b) antibody followed by immunoblotting with an anti-Myc or anti-Flag antibody respectively. Pan 14-3-3 was used as loading control.

Next, we wanted to see whether the mutations found in PARK15 patients affected the binding to PI31. I therefore overexpressed the FBXO7 mutants together with the Flagtagged PI31 and performed co-immunoprecipitation analysis (Figure 3.2.2). In this experiment PI31 interacted to a similar degree with the wild type FBXO7 protein as compared to its mutant variants. 


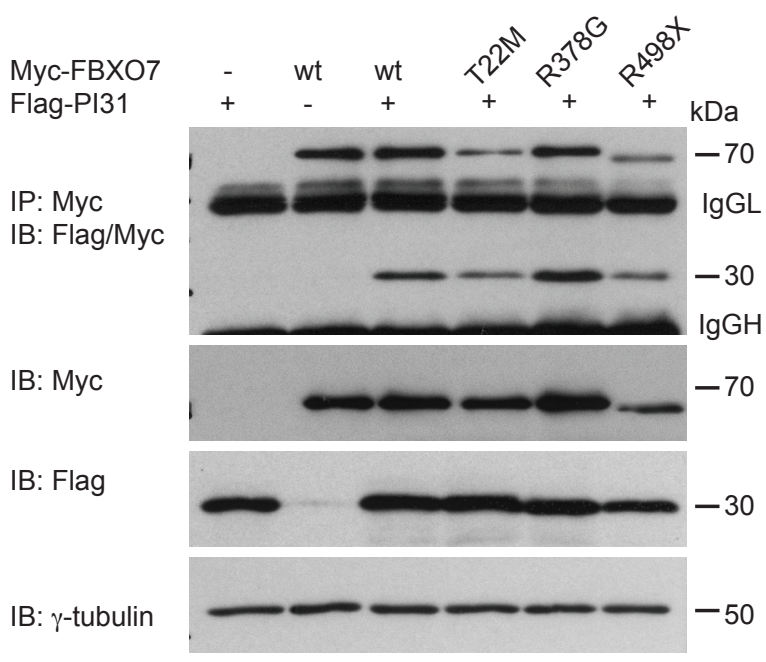

Figure 3.2.2 Mutant FBXO7 proteins interact with PI31. Myc-tagged $\mathrm{FBXO} 7$ and mutant variants found in PARK15 patients were coexpressed with Flag-tagged PI31 in HEK293T cells. Cells lysates were subjected to immunoprecipitation with the Myc antibody followed by immunoblotting with the Flag or the Myc antibody. $\gamma$-tubulin was used as loading control.

The association of FBXO7 with the proteasome has previously been observed in a massspectrometry study (Bousquet-Dubouch et al., 2009). To confirm this association in mouse brain, I conducted a GST pulldown analysis (Figure 3.2.3). I isolated the $26 \mathrm{~S} / 30 \mathrm{~S}$ proteasome via GST-tagged Rad23 that bind to its regulatory 19S subunit and performed SDS-PAGE analysis. After immunoblotting for FBXO7 and PI31, I found both to be associated with the proteasome.

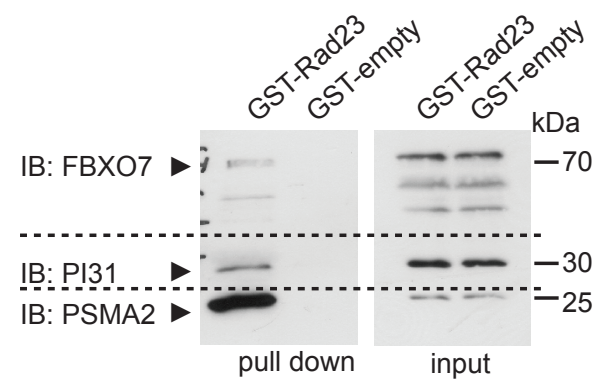

Figure 3.2.3 FBXO7 and PI31 interact with the proteasome. Brain lysates from wild-type adult C57BL/6N mice were subjected to GST-bound Rad23 or empty GST protein. GST proteins and bound proteasome were precipitated by glutathione sepharose beads and eluted. Samples were immunoblotted with FBXO7 or PI31 antibodies. PSMA2 was used as proteasomal control.

To conduct further analyses on FBXO7, PI31 and PSMA2 and their interaction, I generated shRNA constructs targeting their mRNA. I generated two functional shRNA variants for each mRNA and one non-functional construct to be used as a negative control. The FBXO7 shRNA constructs 1 and 2 were designed by Madhuvanti Kannan and the PSMA2 shRNA control was designed by David Brockelt. All functional constructs efficiently knocked down their target protein in HEK293T cells (Figure 3.2.4). 


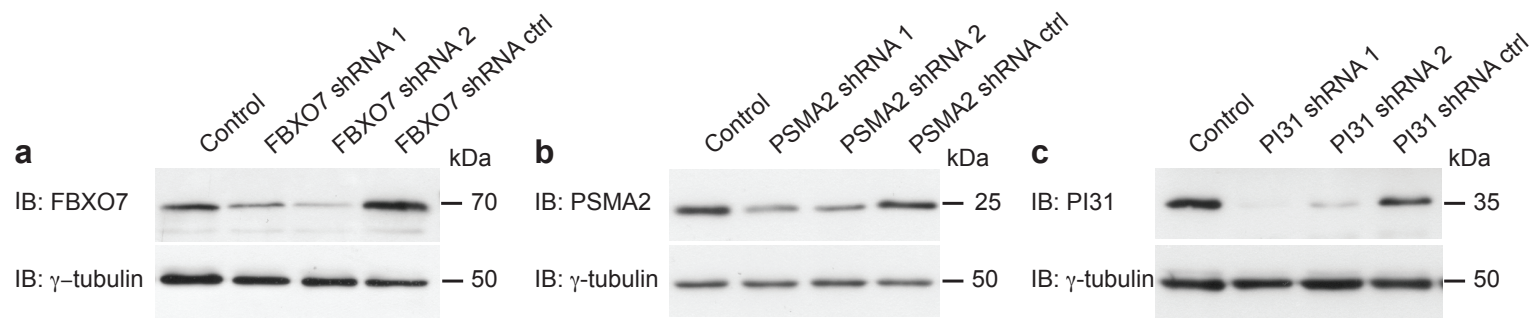

Figure 3.2.4 Validation of shRNA constructs for FBXO7, PSMA2 and PI31. HEK293T cells were transfected with shRNA plasmids targeting FBXO7 (a), PSMA2 (b) or PI31 (c). Cells were lysed and lysate subjected to immunoblotting with the FBXO7 (a), PSMA2 (b) or PI31 (c) antibody. $\gamma$-tubulin was used as loading control.

PSMA2 is an interaction partner of FBXO7, whereas PI31 interacts with both FBXO7 and the proteasome. I therefore tested the hypothesis that PI31 directly interacts with PSMA2. I either overexpressed or knocked down FBXO7 in HEK293T cells and co-expressed Flag-tagged PI31 and Myc-tagged PSMA2. Subsequently, I used the Myc antibody to pull down PSMA2 and immunoblotted for FBXO7 or Flag (PI31). PI31 was only co-precipitated in the presence of $\mathrm{FBXO7}$ showing that $\mathrm{PI} 31$ interacts with PSMA2 through FBXO7 (Figure 3.2.5a).

FBXO7 overexpression in the presence of PSMA2 was shown by David Brockelt to increase a potential ubiquitination smear on PSMA2. Further ubiquitination experiments of his indicated that FBXO7 ubiquitinates PSMA2. To determine if PI31 could influence this process, I co-expressed GFP-PSMA2 and Myc-FBXO7 in the presence or absence of PI31. In the absence of PI31 the potential ubiquitination smear on PSMA2 became visibly stronger suggesting that PI31 negatively influences the possible ubiquitination of PSMA2 by FBXO7 (Figure 3.2.5b). 


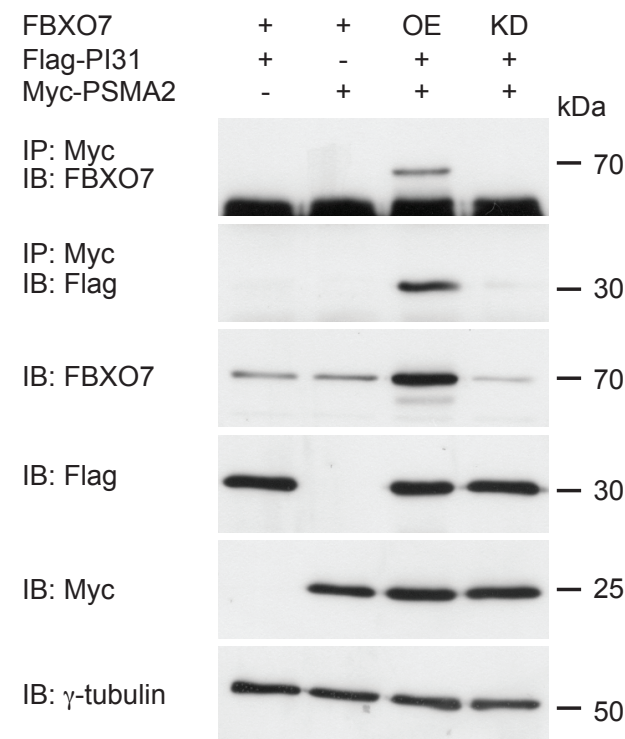

b

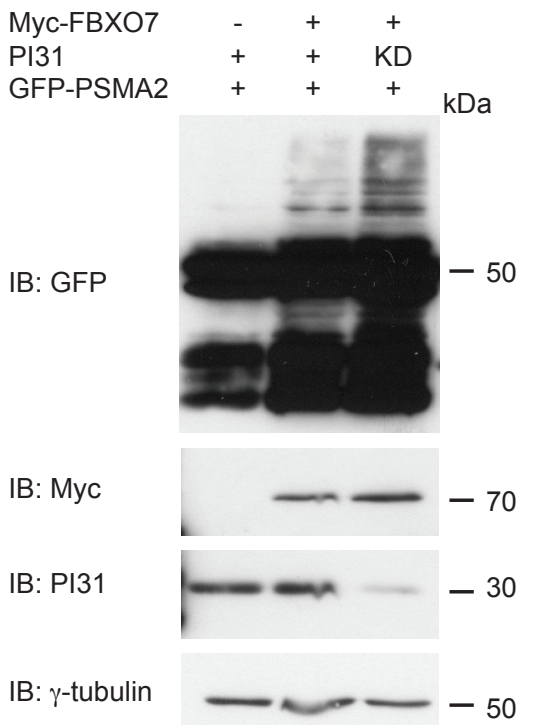

Figure 3.2.5 PI31 interacts with PSMA2 through FBXO7 and may affect its ubiquitination. Untagged FBXO7 and Flag-PI31 were co-expressed with Myc-PSMA2 in HEK293T cells (a). Cells were lysed and lysate subjected to co-immunoprecipitation with a Myc antibody followed by immunoblotting with the FBXO7 or Flag antibody. Myc-tagged FBXO7 was co-expressed with GFP-PSMA2 and shRNA against PI31 or control vector in HEK293T cells (b). Cells were lysed and lysate subjected immunoblotting with the GFP, Myc or PI31 antibody. $\gamma$-tubulin was used as loading control. $\mathrm{OE}=$ overexpression, $\mathrm{KD}=\mathrm{knockdown}$.

With the proteasomal interaction firmly established, we investigated how FBXO7 regulated proteasomal function. I took advantage of the shRNA constructs and first knocked down PSMA2 in HEK293T cells as validation of the RNA silencing and subjected the lysate to analysis with an artificial substrate for the chymotrypsin-like activity of the proteasome (Figure 3.2.6a). The knockdown of PSMA2 led to an approximate 20\% reduction of proteasome activity. I then carried out a similar experiment with shRNA targeting FBXO7 (Figure 3.2.6b). Interestingly, I observed that knockdown of FBXO7 also led to a $20 \%$ lower proteasome activity for the most potent FBXO7 shRNA. To control for the possibility that this effect was due to FBXO7s interaction with PI31, I knocked down PI31 in the same manner (Figure 3.2.6c). This knockdown however, had no effect on proteasome activity despite a highly efficient reduction in PI31 protein levels. This strengthened the hypothesis that FBXO7 itself is important for proteasomal function. 

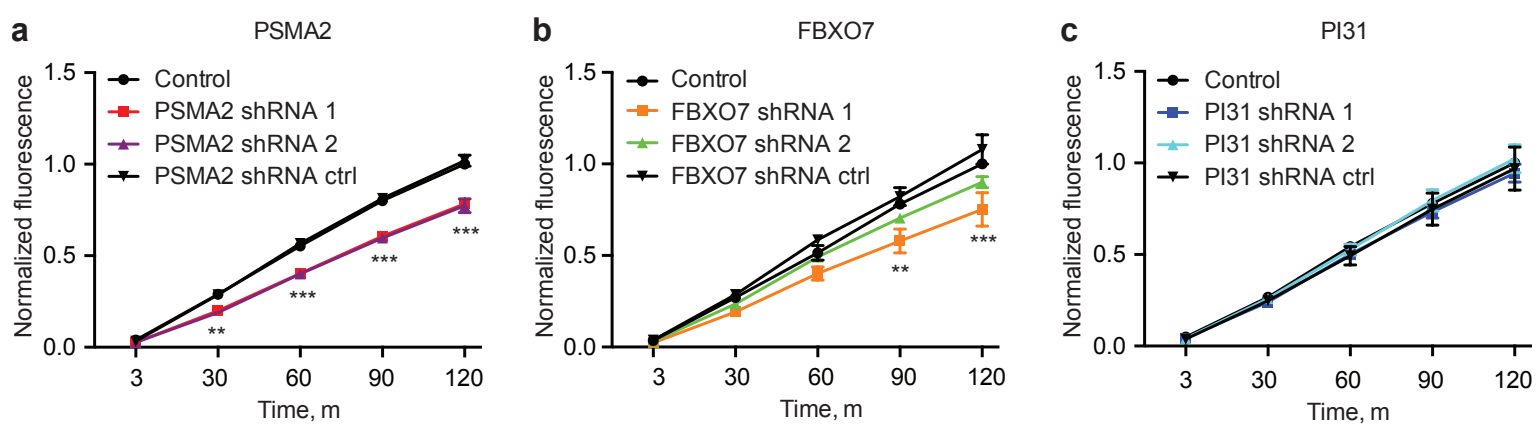

Figure 3.2.6 Knockdown of PSMA2 and FBXO7, but not PI31, lowers proteasome activity. Lysates of HEK293T cells, transfected with control vector or shRNA targeting PSMA2 (a), FBXO7 (b) or PI31 (c), were subjected to proteasome activity assay. Data represent mean \pm SEM of three independent experiments (ANOVA, ${ }^{* *} p<0.01,{ }^{* * *} p<0.001$ ).

To determine if FBXO7 could increase proteasome activity if overexpressed, I continued the same analysis with overexpressed Myc-FBXO7, but saw no difference in proteasome activity as compared to control vector (Figure 3.2.7a). The same was true for overexpression of PI31 (Figure 3.2.7b).
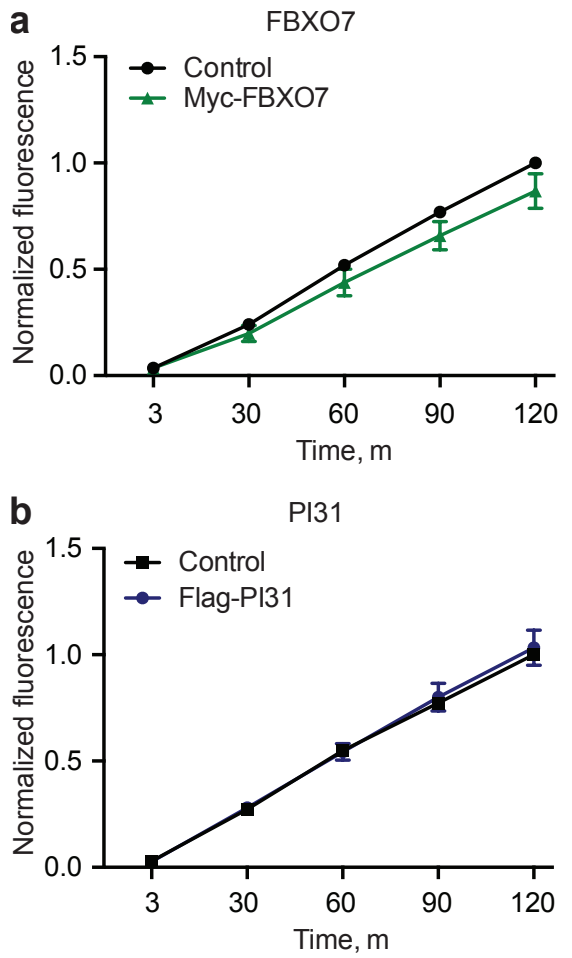
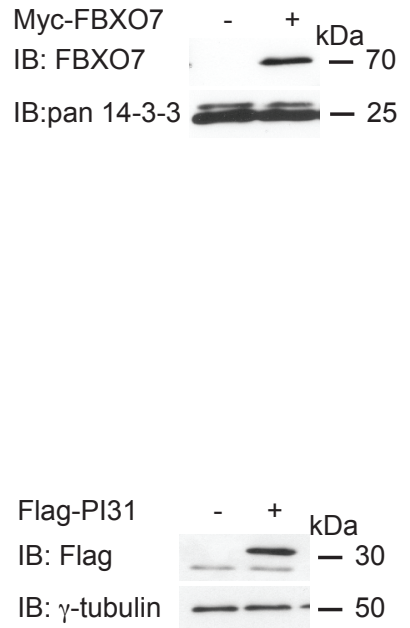

Figure 3.2.7 Overexpression of FBXO7 and PI31 do not influence proteasome activity. Lysates of HEK293T cells, transfected with Myc-FBXO7 (a) or Flag-PI31 (b) together with control vector, were subjected to chymotrypsin-like proteasome activity assay. Data represent mean \pm SEM of three independent experiments (ANOVA). Immunoblots show overexpression of MycFBXO7 (a) and Flag-PI31 (b). Pan 14-3-3 and $\gamma$-tubulin were used as loading controls. 
With these experiments, I establish FBXO7 as a proteasomal interactor and a positive regulator of proteasome activity. I also show that this function is independent of its interaction with PI31. However, as ubiquitination of PSMA2 seems enhanced in absence of $\mathrm{PI} 31$, it could serve as a modulator of FBXO7.

\subsection{Characterization of the novel FBXO7 knockout mouse model}

The study of neurological disease cause and treatment depend heavily upon good animal model systems. Earlier rodent models of recessively inherited Parkinsonism have shown only subtle or variable symptoms (Blesa and Przedborski, 2014). Therefore we sought to establish the FBXO7 knockout (KO) mouse as a model of early-onset ParkinsonianPyramidal syndrome with the aim to use this model to investigate the function of neuronal FBXO7 in vivo.

\subsubsection{Generation of FBXO7 conventional knockout mice}

FBXO7 mutations show a recessive inheritance pattern in affected families and fibroblasts from one affected family showed a reduction in FBXO7 protein levels (Di Fonzo et al., 2009). Hence, we speculated that PARK15 symptoms arise due to a loss of function of the FBXO7 protein. We wanted to model this functional loss in the entire body as well as in specific brain tissue to understand the neuronal function of FBXO7. We purchased embryonic stem cells from EUCOMM, project 23037, with a dual conventional and conditional knockout potential. These ES cells from C57/BI6N mice harbored a construct where two FRT sites as well as three loxP sites flanked the exon 4 of FBXO7 (Figure 3.3.1). In addition, the construct harbored a neomycin cassette for ES cell selection and a $\beta$-gal reporter cassette for expression analysis.

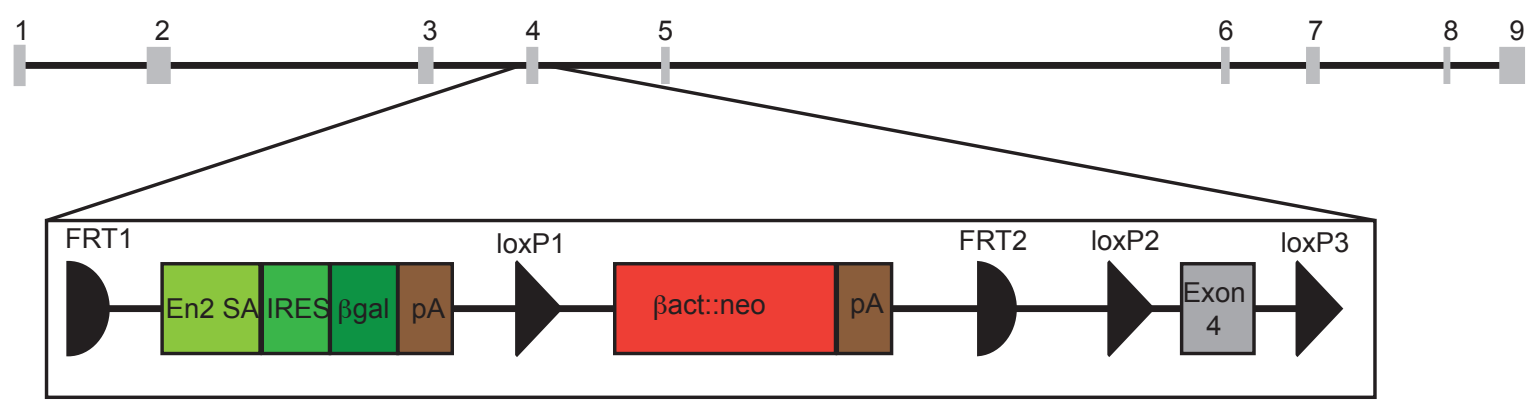

Figure 3.3.1 Targeting cassette for homologous recombination of FBXO7. A gene insertion cassette was introduced via homologous recombination into ES cells from C57/BI6N mice. $\beta g a l=L a c Z$ reporter gene; $\beta$ act:neo=Neomycin selection marker; $p A=$ polyA tail; $F R T=f l i p$ 
recombinase recognition sequence; loxP=cre recombinase recognition sequence; IRES=Internal recombination sequence; En2 SA=splice acceptor sequence for En2.

Nicola Schwedhelm-Domeyer cultured the ES cells, which were injected into blastocysts and then transferred into pseudopregnant NMRI mice. Chimeras were further bred with wild type C57BL/6N mice to obtain germline transmission. The resulting offspring were then bred to homozygosity and further bred to an Ella-cre mice line, which targets the Cre expression to germline cells. This recombination left a truncated form of $F B X O 7$ consisting of the first three exons followed by the $\beta$-gal cassette and a stop codon. This line was then further bred to wild type C57BL/6N mice to eliminate the cre recombinase gene and then bred to homozygosity. To verify the loss of $F B X O 7$, I ran genotyping PCR on tail tissue samples (Figure 3.3.2a). Nicola Schwedhelm-Domeyer analyzed mRNA expression by qPCR (Figure 3.3.2b) and I performed immunoblotting analyses on brain tissue to confirm loss of FBXO7 protein (Figure 3.3.2c).
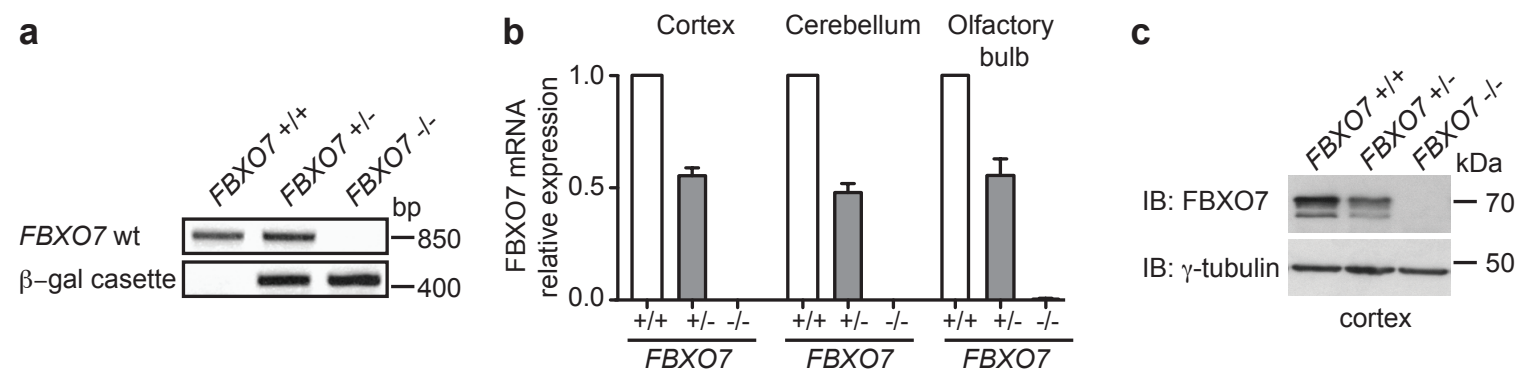

Figure 3.3.2 Disruption FBXO7 gene, mRNA and protein expression in FBXO7-/- mice. Tail biopsies from $F B X O 7+/+,+/-$ and -/- littermates were subjected to PCR analysis with primers recognizing wild type $F B X O 7$ or $\beta$-gal cassette (a). RNA isolated from wild type, heterozygous and homozygous FBXO7 littermates at P18 was reverse transcribed and subjected to QPCR analysis with primers for $\mathrm{FBXO} 7$ and $\beta$-actin, the latter served as housekeeping control (b). Data represent mean \pm SEM of three independent experiments (ANOVA, ${ }^{* * *} p<0.001$ ). Whole brain lysates of P18

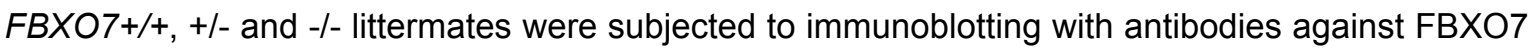
and $\beta$-gal. $\gamma$-tubulin was used as loading control.

\subsubsection{FBXO7-/- mice show retarded growth and premature death}

FBXO7 is a subunit of the cullin1-based E3 ligase. The deletion of core components including cullin 1 and $\mathrm{Rbx} 1$ e.g. leads to a very early embryonic lethality in mice (Tan et al., 2009; Zhou et al., 2013). We therefore sought to determine the importance of FBXO7 for the organism. The ratio of born pups and survival up until genotyping (P18) was, however, close to Mendelian ratio, 1:2,2:1 (+/+:+/-:-/-) (Table 3.3.1). Hence I concluded that there is little or no embryonic lethality in this line. 
Table 3.3.1 Ratio of mice pups born to $F B X 07+/-$ parents. Pups from 15 litters were genotyped between the age of $\mathrm{P} 5$ and $\mathrm{P} 18$ and ratio of genotypes calculated.

\begin{tabular}{|c|c|c|c|c|c|c|c|c|}
\hline \multirow[b]{2}{*}{ Litter } & \multicolumn{4}{|c|}{ Male } & \multicolumn{4}{|c|}{ Female } \\
\hline & FBXO7+/+ & FBXO7+/- & FBX07-/- & Total & FBXO7+/+ & FBXO7+/- & FBX07-/- & Total \\
\hline 1 & 2 & 3 & 0 & 5 & 1 & 2 & 2 & 5 \\
\hline 2 & 1 & 3 & 1 & 5 & 1 & 1 & 0 & 2 \\
\hline 3 & 0 & 2 & 0 & 2 & 1 & 4 & 3 & 8 \\
\hline 4 & 2 & 4 & 0 & 6 & 0 & 1 & 2 & 3 \\
\hline 5 & 0 & 2 & 1 & 3 & 1 & 1 & 0 & 2 \\
\hline 6 & 2 & 2 & 1 & 5 & 2 & 2 & 0 & 4 \\
\hline 7 & 1 & 3 & 0 & 4 & 0 & 2 & 1 & 3 \\
\hline 8 & 1 & 1 & 1 & 3 & 0 & 1 & 1 & 2 \\
\hline 9 & 1 & 2 & 1 & 4 & 0 & 2 & 1 & 3 \\
\hline 10 & 0 & 2 & 1 & 3 & 1 & 2 & 1 & 4 \\
\hline 11 & 1 & 3 & 0 & 4 & 1 & 0 & 2 & 3 \\
\hline 12 & 1 & 1 & 1 & 3 & 1 & 1 & 0 & 2 \\
\hline 13 & 1 & 1 & 0 & 2 & 0 & 2 & 1 & 3 \\
\hline 14 & 0 & 0 & 1 & 1 & 2 & 1 & 2 & 5 \\
\hline 15 & 1 & 2 & 1 & 4 & 0 & 2 & 0 & 2 \\
\hline Sum & 14 & 31 & 9 & 54 & 11 & 24 & 16 & 51 \\
\hline Ratio & 0.259 & 0.574 & 0.167 & & 0.216 & 0.471 & 0.314 & \\
\hline
\end{tabular}

From the initial litters, I realized that pups did not survive longer than 25 days with a mean life expectancy of 21 days (Figure 3.3.3). We therefore kept homozygous mice no longer than P18.

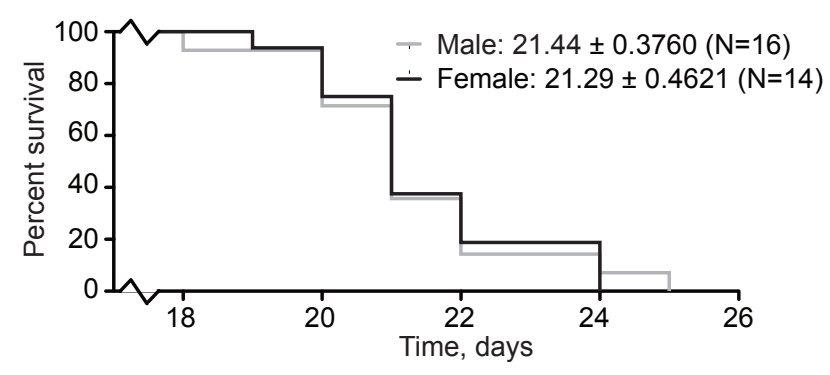

Figure 3.3.3 FBXO7-/- mice show premature lethality. Death day of $\mathrm{FBXO7+/+,} \mathrm{+/-} \mathrm{and} \mathrm{-/-} \mathrm{mice} \mathrm{of}$ mixed gender was noted and is shown as a Caplan-Meyer survival curve with a mean expiry day of $P 21$. Data represent mean \pm SEM.

Based on life expectancy, we conducted all analyses at P5 and P18. I sacrificed all pups that were too weak to move and did not include them in the analysis. Both brain and bodyweight showed a tendency for FBXO7-/- pups to be smaller already at P5, but this did not reach significance (Figure 3.3.4a,b). When we conducted the same measurements at $\mathrm{P} 18$, it became clear that the brain weight was diminished, and the pups were only half the size of their littermates (Figure 3.3.4c-e). 

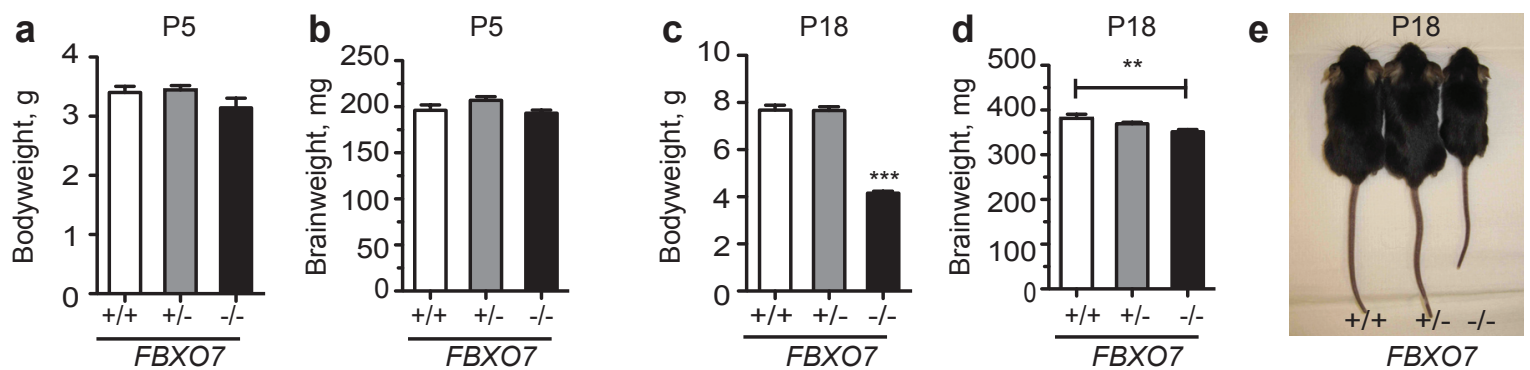

FBX07

Figure 3.3.4 FBXO7 knockout mice show retarded growth. Body (a) and brain weight (b) was measured for $F B X O 7+/+,+/-$ and -/- mice of mixed gender at P5. Body (c) and brain weight (d) of male $F B X O 7+/+,+/-$ and $-/-$ mice were measured at $\mathrm{P} 18 . \mathrm{N}=5-15$ animals per genotype. Data represent mean \pm SEM. (ANOVA, ${ }^{* *} p<0.01,{ }^{* * *} p<0.001$ ). Representative picture of male mice at P18 (e).

\subsubsection{FBX07-/- mice show diminished motor control at P18}

In further analyses, I explored the possibility that the slightly reduced size of the P5 pups was already accompanied by motor problems. For this, I tested the pups' ability to roll onto their stomach when turned on their back. This reflex is important at an early stage and was not changed to a significant degree in the homozygotes (Figure 3.3.5).

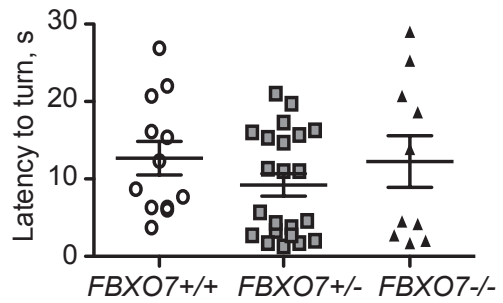

Figure 3.3.5 FBXO7-/- mice show normal surface righting reflex at P5. FBXO7+/+, +/- and -/- mice of mixed gender aged P5 were placed on their back on a flat surface and time to turn was measured three times. Average time is shown. $N>10$ animals per genotype. Data represent mean \pm SEM (ANOVA, $* * * p<0.001)$.

As a morphological characterization I looked at the spine and found FBXO7-/- mice to display slight kyphosis (Figure 3.3.6a,b). I also observed moderate hind limb clasping upon the tail suspension test (Figure 3.3.6c,d). 
a

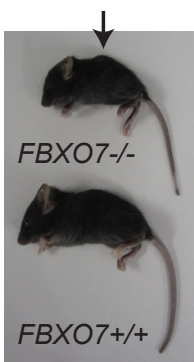

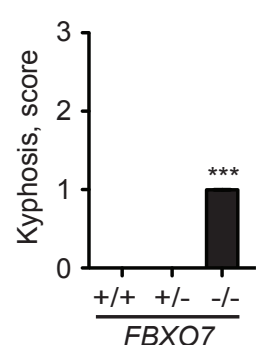

b

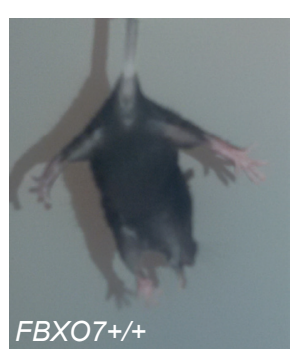

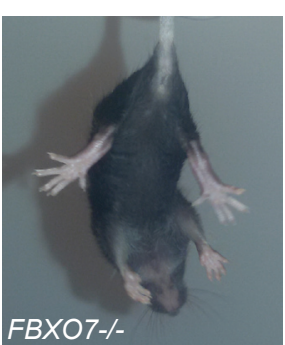

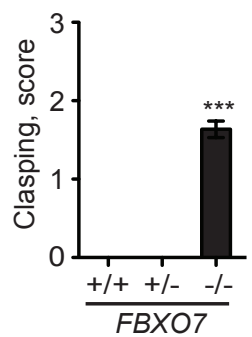

Figure 3.3.6 FBXO7-/- mice display mild kyphosis and hind limb clasping. Male $F B X O 7+/+$, +/- and -/- mice at P18 were scored for kyphosis (a) with 0 indicating normal to 3 indicating severe phenotype. Mice were suspended by the tail for $3 \times 10$ seconds and scored for hind limb clasping in a similar manner from 0 to 3 (b). Representative pictures are shown. $N>10$ animals per genotype. Data represent mean \pm SEM (Kruskal-Wallis non-parametric ANOVA, Dunn's Multiple Comparison test, $\left.{ }^{* * *} p<0.001\right)$.

At $\mathrm{P} 18$, I subjected the pups to more rigorous motor testing to assess for motor function impairment. Because of the small size and young age of the animals, I first conducted a set of experiments in their home cage and in a small open field. The gait score was based on the characteristics of whether the young mouse was able to keep its body above the ground, if it showed tremor or was walking in a crooked line. Initally, the homozygots only tended to keep their feet in a more outward poise and walked with a slightly more hunched back (Figure 3.3.7a). Their ambulation was then further assessed in the open field, where the young mice were allowed to walk freely in a square $28 \times 28 \mathrm{~cm}$ open field. The arena was divided into 16 squares and the number of crossings were counted and divided into central and peripheral crossings. The total number of crossings was rather elevated than diminished for the homozygotes indicating normal ambulation level with slight hyperactivity in some individuals (Figure 3.3.7b). In addition, the ratio of central versus peripheral crossings was analyzed to look at anxiety state of the mice (Figure 3.3.7c). Here it became clear that a majority of the homozygotes moved in circles with dispersed repetitive jumping. This made the homozygotes move at the edges of the field rather than in the middle. It was therefore impossible to conclude anything regarding the anxiety state in these mice. 

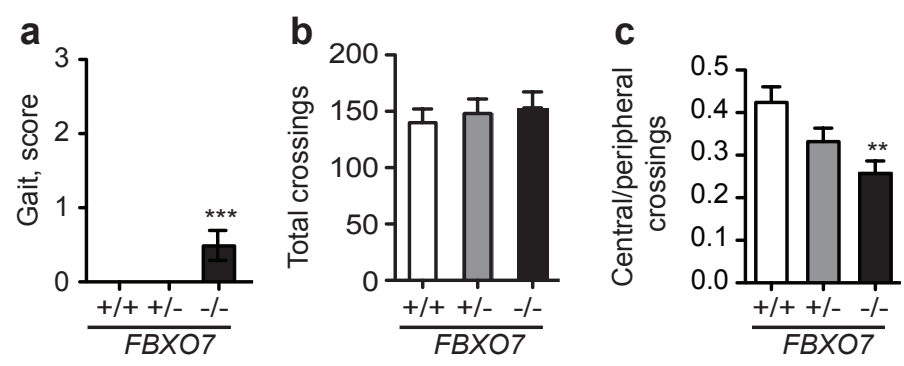

Figure 3.3.7 FBXO7-/- mice display normal ambulation and slight gait aberrations. Male $F B X O 7+/+,+/-$ and $-/-$ mice at $\mathrm{P} 18$ were placed in a $28 \times 28 \mathrm{~cm}$ open field and allowed to move freely. The gait was scored from 0-3 with 0 being normal and 3 worst manifestation of ataxic gait (a). Data represent mean \pm SEM (Kruskal-Wallis non-parametric ANOVA, Dunn's Multiple Comparison test, $\left.{ }^{* * *} p<0.001\right)$. The open field was divided into 16 squares and total number of line crossings counted (b). The number of central square crossings was divided by total number of crossings (c). Data represent mean \pm SEM (ANOVA, Bonferronis Multiple Comparison test, $\left.{ }^{* *} \mathrm{p}<0.01\right) . \quad \mathrm{N}=15-20$ animals per genotype.

To assess their balance, I let the young mice walk on the ledge of their cage and scored them from 0 (normal) to 3 (unable to walk on ledge) according to their coordination (Figure 3.3.8a). Most of the homozygotes were not able to walk on the ledge due to multiple slips and many fell off the ledge. To test their grip strength, I placed the mice on the middle of an $80 \mathrm{~cm}$ long wire suspended above ground (Figure 3.3.8b). While most wild types and heterozygotes had no problems holding on to the wire for 30 seconds or climb over to the platform on either end, the homozygotes were barely able to hold on to the wire. Since ambulation seemed normal in an open field, I set out to challenge the pups on the rotarod (Figure 3.3.8c). Here, the mice walked on an accelerating rod from 4-40 rpm over 300 seconds. Again the homozygotes performed significantly worse than their littermates. The pole test has been used to assess symptoms of dopaminergic neuron loss (Ogawa et al., 1985), so I placed the mice on a pole facing upwards and recorded the time to turn and climb down (Figure 3.3.8d). Here the homozygotes adopted a strategy of grip and fall, so they slid down the pole rather than climb.
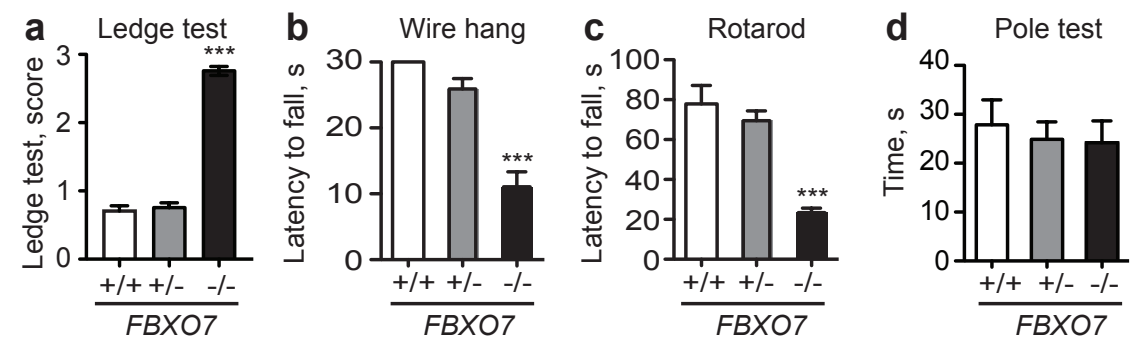

Figure 3.3.8 FBXO7-/- mice show diminished motor control. FBXO7+/+, +/- and -/- male mice at $\mathrm{P} 18$ were subjected to a ledge test (a). Mice were placed on the ledge of home cage and scored 
according to performance with 0 being able to cross with no problem and 3 not able to cross. Data represent mean \pm SEM (Kruskal-Wallis non-parametric ANOVA, Dunn's Multiple Comparison test, $\left.{ }^{* * *} p<0.001\right)$. They were also placed on a wire and time hanging before fall recorded (b). Mice were placed on an accelerating rotarod (4-40 rpm in $5 \mathrm{~min}$ ) and latency to fall recorded (c). Mice were placed face up on a $30 \mathrm{~cm}$ long pole and latency to turn and climb down was recorded. Data represent mean \pm SEM (ANOVA, Bonferronis Multiple Comparison test, ${ }^{* * *} p<0.001$ ). $\quad N=15-20$ animals per genotype.

Based on these experiments, I conclude that $F B X O 7-/-$ mice are viable until postnatal day 18 , but then die prematurely. The phenotypic analysis I conducted on P18 conventional FBXO7-/- mice revealed a significant reduction in body and brain size. This was accompanied by reduced muscle strength and weakness, but relatively normal ambulation with slight hyperactivity and stereotypic movements. This shows that loss of FBXO7 is detrimental to normal development of mice, but does not restrict the dysfunction to the nervous system.

\subsubsection{Heterozygous FBXO7 mice show normal behavior at 6 and 12 months of age}

Since loss of FBXO7 resulted in such a dramatic phenotype in mice, we asked if heterozygote mice would show a similar phenotype at a later stage due to haploinsufficiency of FBXO7. I therefore ran a battery of behavioral tests on the $\mathrm{FBXO7+/-}$ mice and their wild type littermates at 6 and 12 months of age. I first assessed their weight and hind limb clasping through the tail suspension test (Figure 3.3.9a,b) and found no difference between genotypes at either age. The same held true for muscle strength as shown by the inverted grid test (Figure 3.3.9c)
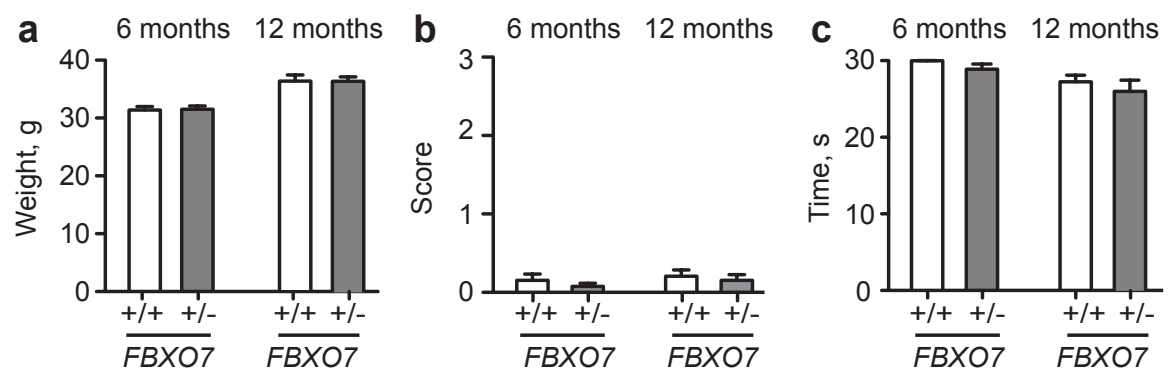

Figure 3.3.9 FBXO7+/- mice show normal body characteristics and muscle strength. $F B X O 7+/+(\mathrm{N}=18)$ and $+/-(\mathrm{N}=15)$ mice were weighed at different ages $(\mathrm{a})$. Data represent mean \pm SEM (t-test). The same mice were suspended by the tail (b) and scored for hind limb clasping. They were also placed on an inverted cage lid and time until fall was measured (c). Data represent mean \pm SEM (Mann-Whitney non-parametric t-test).

I recorded the total track length of the mice, which spent 7 minutes in the open field, and found no difference ambulation between groups (Figure 3.3.10). 


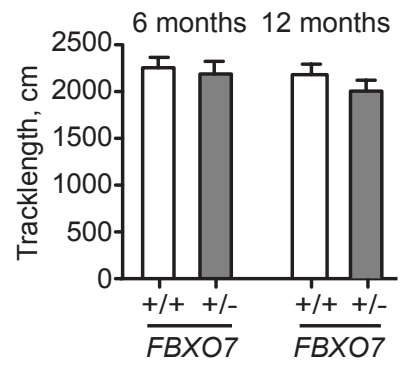

Figure 3.3.10 FBXO7+/- mice show normal ambulation. $F B X O 7+/+$ $(\mathrm{N}=18)$ and $+/-(\mathrm{N}=15)$ mice were allowed to move freely in a circular open field $60 \mathrm{~cm}$ in diameter. Movement was recorded by the Viewer software. Total track length after 7 minutes was measured. Data represent mean \pm SEM (t-test).

To measure general anxiety in these mice, I first looked at the time the mice spent in different zones in the open field (Figure 3.3.11a). Then I performed an elevated plus maze test and measured the fraction of time the animals spent in the open arms of the maze (Figure 3.3.11b) Both tests showed that $F B X O 7+/-$ mice have normal anxiety levels.
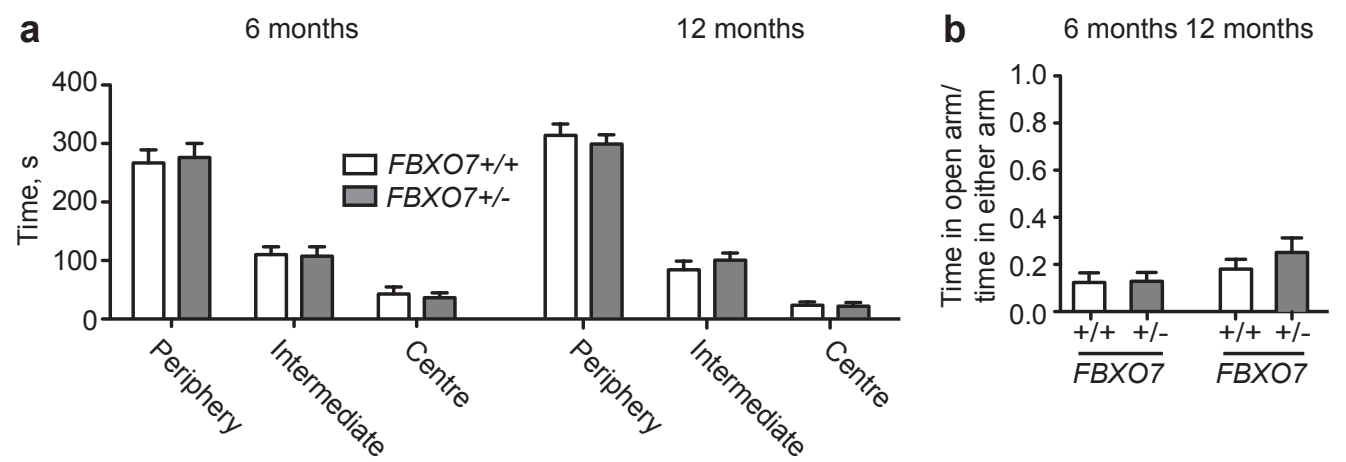

Figure 3.3.11 FBXO7+/- mice show normal anxiety levels. $F B X O 7+/+(\mathrm{N}=18)$ and $+/-(\mathrm{N}=15)$ mice were allowed to move freely in a circular open field $60 \mathrm{~cm}$ in diameter (a). Time spent in each zone during 7 minutes was measured. Mice were placed in an elevated plus maze with two open and two closed arms for 5 minutes (b). Time spent in open arms during 5 minutes is shown as ratio to time spent in either arm. Data represent mean \pm SEM (t-test).

I further examined the heterozygous mice on the rotarod to measure their general motor function using a learning paradigm (Figure 3.3.12a). Then I tested balance and coordination with the balance beam test (Figure 3.3.12b,c). In neither of these tests did the heterozygous mice perform worse than their wild type littermates. Also the pole test as a sensitive test for loss of dopaminergic neurons, revealed no difference for the heterozygous animals (Figure 3.3.12d). 

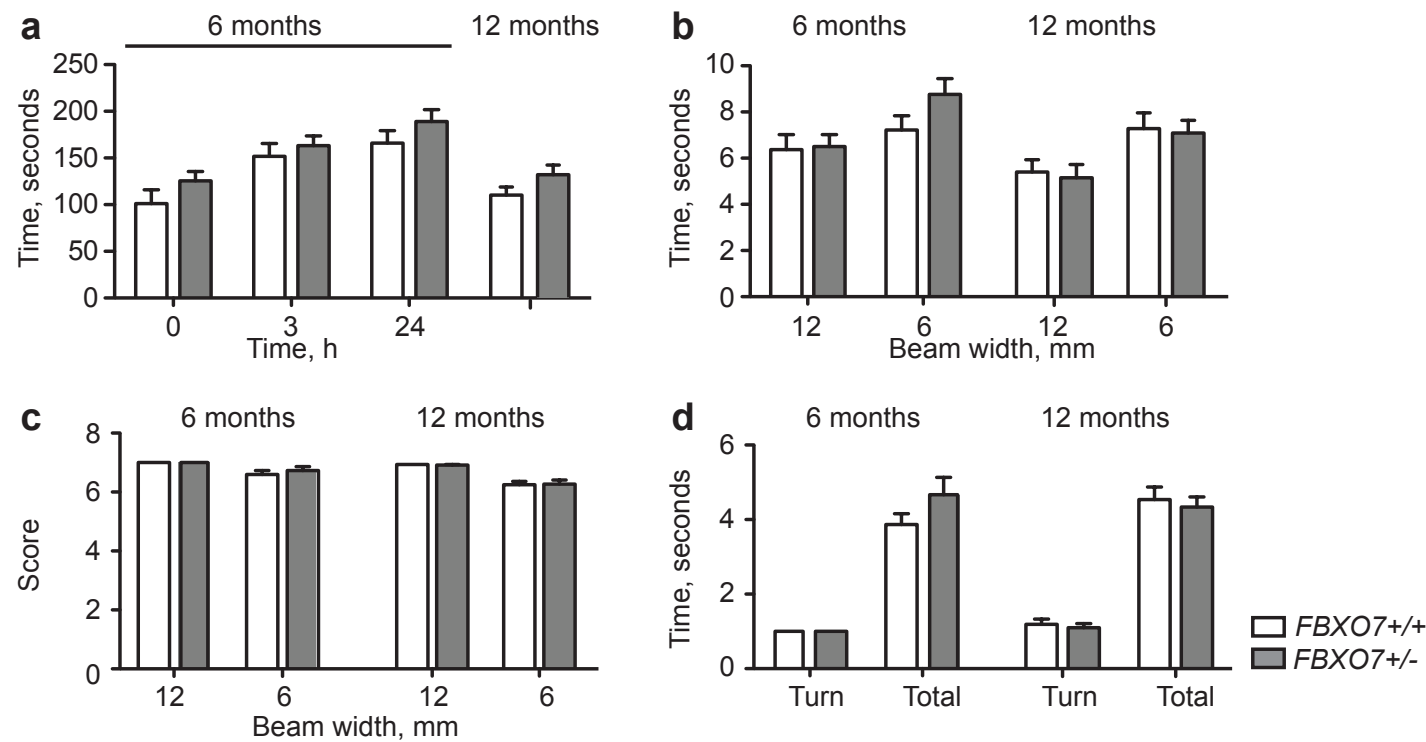

Figure 3.3.12 FBXO7+/- mice show normal motor function. $F B X O 7+/+(N=18)$ and $+/-(N=15)$ mice were placed on a rotarod and exposed to an accelerating protocol from 4-40 rpm for 5 minutes (a). Latency to fall was measured. Mice were placed on a balance beam of either 12 or 6 $\mathrm{mm}$ width and $80 \mathrm{~cm}$ length and encouraged to cross (b,c). Time to cross (b) as well as coordination score (c) was measured. Mice were placed face up on a $50 \mathrm{~cm}$ tall wooden pole and encouraged to climb down (d). Time to turn, as well as time to descend, were recorded. Data represent mean \pm SEM (a,b: t-test, c,d: Mann-Whitney non-parametric t-test).

As the olfactory system is often the first to show defects in Parkinson's patients (Haehner et al., 2011), I performed an olfaction test on the 6 and 12 months old FBXO7+/- mice (Figure 3.3.13). Here mice had to find a buried treat using smell. Also here I found no difference between the heterozygous and the wild type mice.

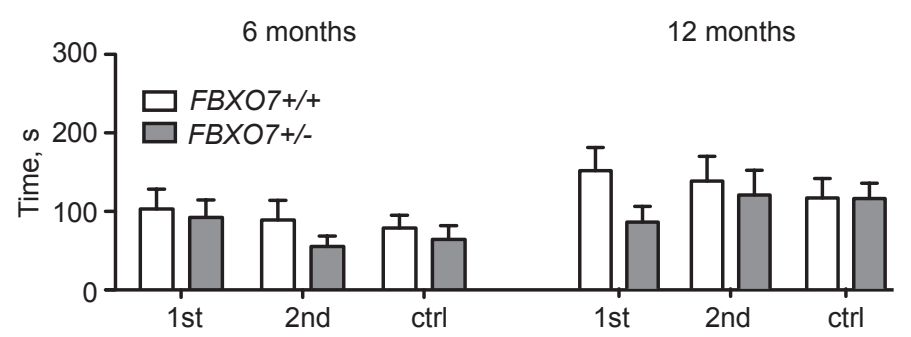

Figure 3.3.13 FBXO7+/- mice show normal olfaction. FBXO7+/+ $(\mathrm{N}=18)$ and $+/-(\mathrm{N}=15)$ mice were placed in a cage with a chocolate chip buried in the bedding in two different corners. On control trial the chip was placed in the middle of the cage on top of the bedding. Time to retrieve treat was measured. Data represent mean \pm SEM (t-test).

Based on these experiments, I conclude that a mere reduction in FBXO7 levels does not have severe consequences for the behavior of the mice up until 12 months of age. 


\subsubsection{FBXO7-/- mice show normal dopaminergic neuron morphology and function}

To evaluate whether dopaminergic neurons are affected by ubiquitous loss of FBXO7, I subjected coronal sections of P18 animals to immunostaining with a tyrosine hydroxylase antibody and Nissl staining (Figure 3.3.14). Every fourth section was stained and neurons counted in a stereological manner. No loss of neurons in the substantia nigra was found at P18.
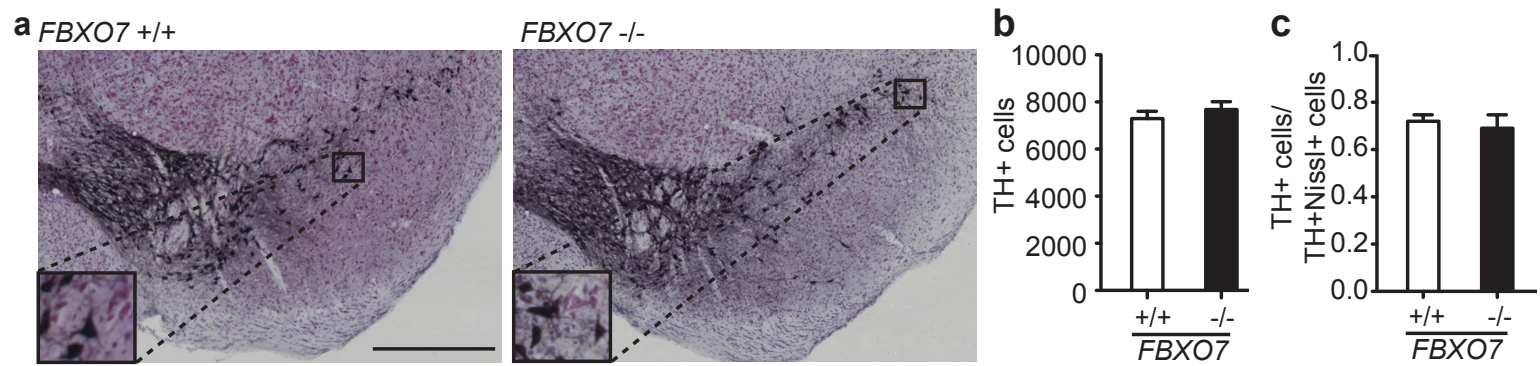

Figure 3.3.14 FBXO7-/- mice show no substantial loss of dopaminergic neurons at P18. Coronal cryo-sections from $F B X O 7+/+(\mathrm{N}=3)$ and FBXO7-/- $(\mathrm{N}=3)$ mice were stained with an antibody directed against tyrosine hydroxylase and then subjected to Nissl-staining (a). Every fourth section was counted and quantified in a stereological manner with a reading frame of 50x50 $\mu \mathrm{m}$. The total number of estimated TH positive neurons (b) as well as the ratio to Nissl positive cells (c) was counted. Data represent mean \pm SEM (t-test).

I then proceeded to look at whether axon terminals in the striatum were affected, by immunoblotting for dopaminergic markers. I used antibodies against tyrosine hydroxylase (TH) and dopamine transporter (DAT) on striatal lysates from P18 animals (Figure 3.3.15a,b). These were unchanged between the genotypes. The pyramidal symptoms seen in PARK15 patients may arise from a disturbance in projection neurons, hence I analyzed the levels of VGLUT1, a marker of glutamatergic neurons, in both striatum and cortex (Figure 3.3.15c,d). These levels were also unchanged between genotypes. Neither of these tests exclude that other axonal features are affected, hence further analyses are necessary to confirm axonal integrity in these mice. 
Striatum TH

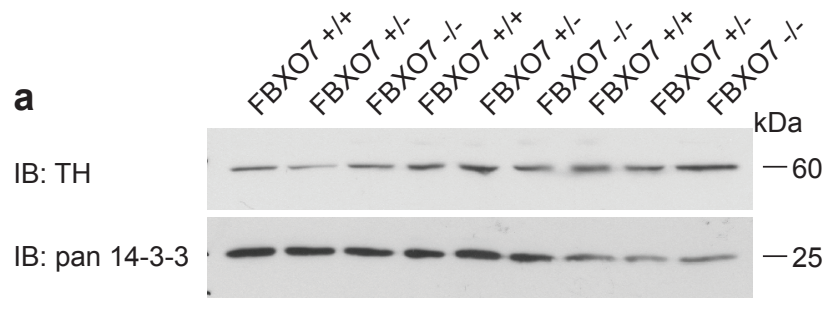

Striatum DAT

b

IB: DAT

IB: pan 14-3-3

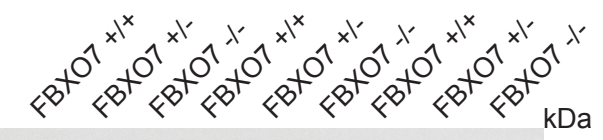

C

IB: VGLUT1

IB: pan 14-3-3

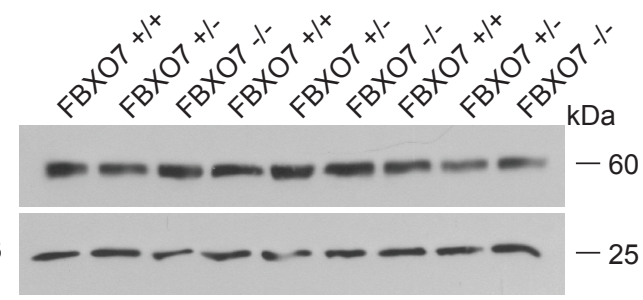

\section{d}

IB: VGLUT1

IB: pan 14-3-3
Striatum VGLUT1

Cortex VGLUT1
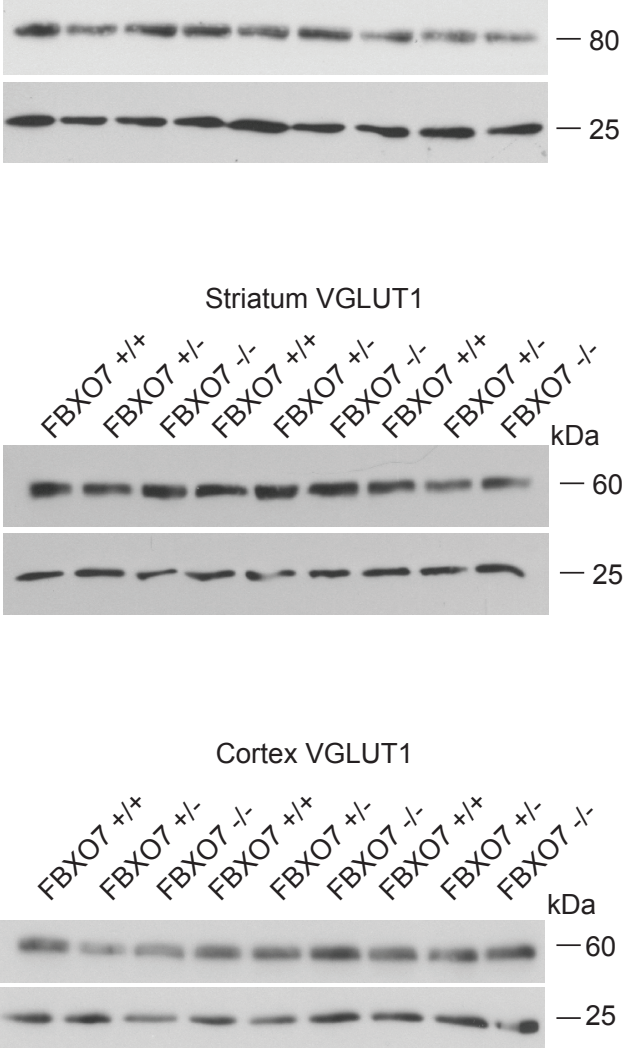
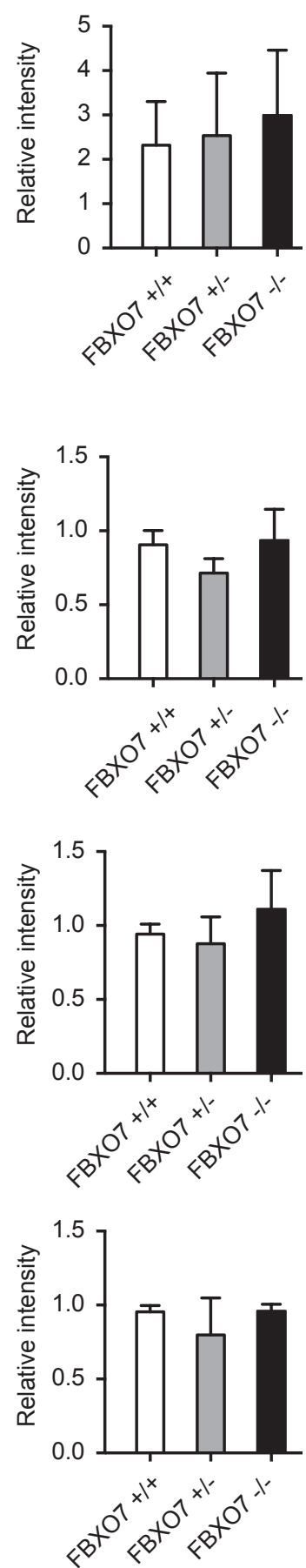

Figure 3.3.15 FBXO7-/- animals show no evident loss of transporters in the striatum. Striatal (a-c) and cortical (d) tissue from P18 FBXO7+/+ (N=3), +/- (N=3) and -/- $(\mathrm{N}=3)$ mice was lysed and subjected to immunoblotting analysis with antibodies against TH (a), DAT (b) and VGLUT1 (c-d). Blots were quantified with the ImageJ software and intensity normalized to loading control (pan 143-3). Values are expressed relative to first $\mathrm{FBXO7+/+}$ sample. Data represent mean \pm SEM (ANOVA). 
To investigate if there was a functional impairment of the dopaminergic system, I prepared samples from freshly lysed striatal tissue of P18 animals. Dr. Lars Tatenhorst then measured the level of dopamine and metabolites by HPLC analysis. The results showed a rather subtle increase in striatal dopamine in FBXO7-/- animals compared to controls, accompanied by a slight decrease of DOPAC levels, which then led to a significant decrease in dopamine metabolism values.
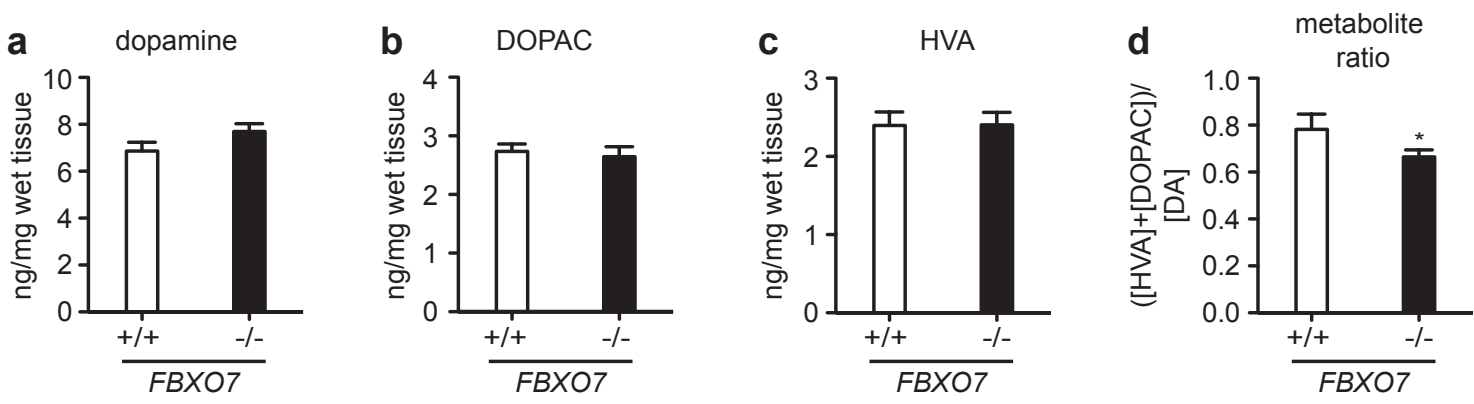

Figure 3.3.16 FBXO7-/- mice show no evident loss of dopamine in the striatum. Striatal tissue from P18 old animals was subjected to HPLC analysis for dopamine (a), DOPAC (b) and HVA (c). Metabolism is shown as ratio of HVA and DOPAC to dopamine (d). N=9 per genotype. Data represent mean \pm SEM (t-test, $\left.{ }^{*} \mathrm{p}<0.05\right)$. HPLC measurements were conducted by Dr. Lars Tatenhorst.

In summary, I could not show any cellular disturbance in the brains of P18 FBXO7-/- mice.

\subsubsection{FBXO7-/- mice show reduced levels of PI31, but no change in proteasome activity or oxidation levels}

Since PI31 and FBXO7 interact, I analyzed what impact loss of FBXO7 had on the PI31 levels in mouse brain tissue. I immunoblotted lysates from cortex of P5 and P16 FBXO7+/+, +/- and -/- mice with a PI31 antibody and found PI31 to be heavily downregulated in FBXO7-/- mice (Figure 3.3.17a). This was not due to a reduced transcription as PI31 mRNA levels in cortex were rather increased (Figure 3.3.17b). 
a

IB: $\beta$-gal
IB: PI31
IB: pan 14-3-3
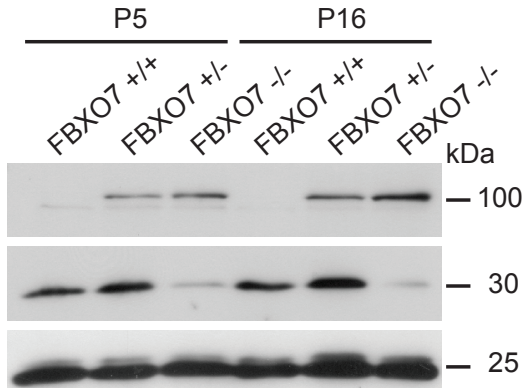

b

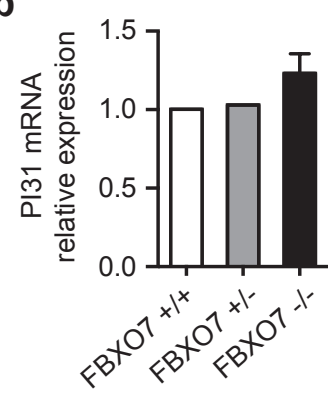

Figure 3.3.17 FBXO7-/- mice show a decrease in PI31 levels. Cortical tissue lysates from P5 and P16 FBXO7+/+, +/- and -/- mice were subjected to immunoblotting with a PI31 antibody (a). $\beta$ gal was used as control for FBXO7 knock-out and pan 14-3-3 as loading control. RNA isolated from cortex of $F B X O 7+/+(n=4),+/-(n=2)$ and $-/-(n=4)$ littermates at $P 18$ was reverse transcribed and subjected to $\mathrm{GPCR}$ analysis with primers for PI31 and $\beta$-actin, the latter served as housekeeping control (b). Data represent mean \pm SEM (ANOVA).

In order to see if I could replicate the findings from HEK293T cells, where I showed that knockdown of FBXO7 lead to a decrease in proteasome activity, I conducted the same proteasome activity assay on cultured cerebellar granule cells from $\mathrm{FBXO} 7+/+$ and $-/-$ mice. Here however, I could not see any difference in proteasome activity between wild type and knockout mice.

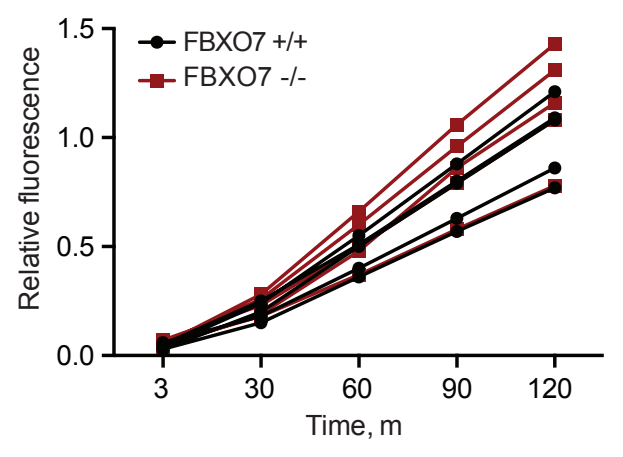

Figure 3.3.18 Cultured cerebellar granule neurons of FBXO7-/- mice show no decrease in proteasome activity. Lysates from cultured cerebellar granule neurons at DIV 16 were subjected to chymotrypsin-like proteasome activity assay. $\mathrm{N}=5$ per genotype (t-test).

FBXO7 has also been linked to mitochondrial regulation (Burchell et al., 2013) and the tight interplay between proteasomal and mitochondrial function is critical in the regulation of reactive oxygen species (ROS) (Sullivan et al., 2004). As FBXO7 is involved in both these processes I investigated if the level of ROS could be changed in FBXO7-/- animals and contribute to an increase in modified carbonyl groups on proteins. I analyzed tissue from cortex of $\mathrm{FBXO7+/+}$ and -/- mice with a carbonyl-derivatizing agent (DNPH) but found no increase in modified proteins in the FBXO7-/- animals (Figure 3.3.19). 


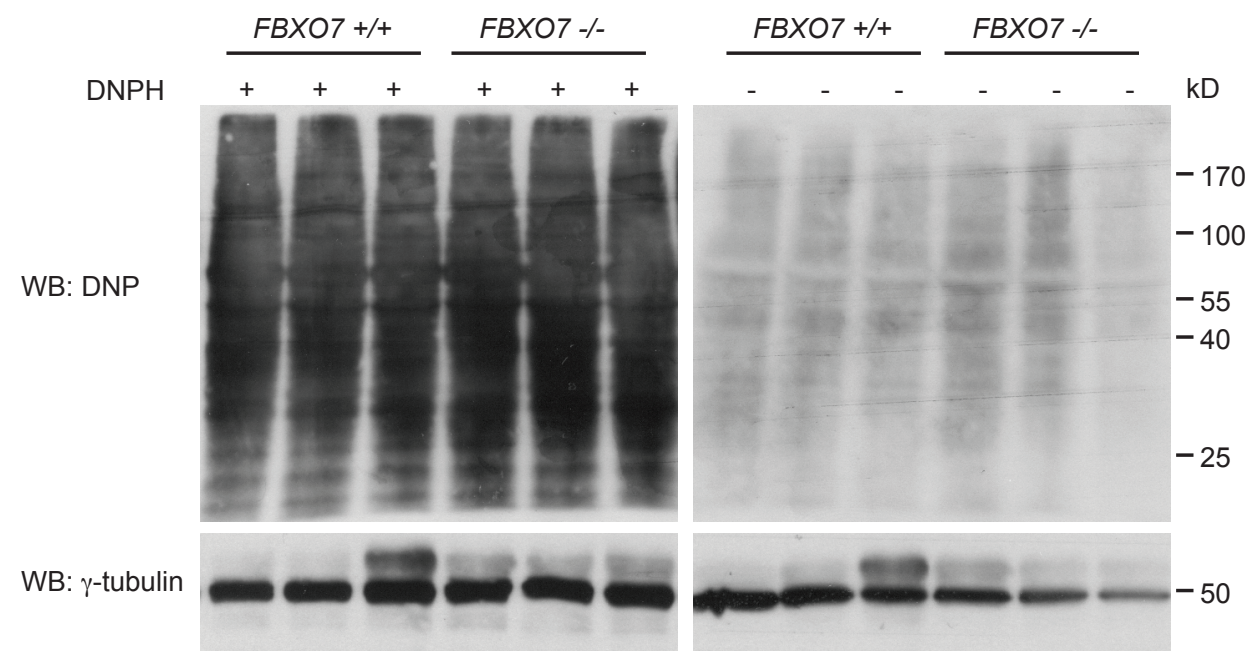

Figure 3.3.19 FBXO7-/- mice show no difference in oxidized protein levels in the cortex. Cortical tissue from P18 FBXO7+/+ and -/- mice was lysed and exposed to 2,4dinitrophenylhydrazine (DNPH, left panel) or control (right panel). The resulting derivatization of carbonyl groups in the protein side chains to 2,4-dinitrophenylhydrazone (DNP-hydrazone) was detected by an anti-DNP antibody. $\gamma$-tubulin served as loading control.

I here show that $\mathrm{PI} 31$ is dependent on FBXO7 as its protein levels are strongly reduced in FBXO7-/- animals. These experiments do not show any obvious disturbance in proteasomal or mitochondrial function of FBXO7-/- animals at P18. David Brockelt could however show that in FBXO7-/- cortical tissue there is a decrease in proteasomal activity similar to what I see in HEK293T cells upon FBXO7 knockdown. Hence, a more thorough set of analyses is necessary to investigate these pathways.

\subsection{NEX-Cre;fl/fl mice show pyramidal symptoms with stereotypic movements and hyperactivity}

\subsubsection{FBXO7 fl/fl mice show normal behavior up to 12 months of age}

To establish the requirement for FBXO7 in neuronal function and to distinguish between the pyramidal and the Parkinsonian phenotype seen in PARK15 patients, we generated two conditional FBXO7 knockout mouse lines. First, mice with the lacZ casette were bred to Flip recombinase mice and the LacZ cassette excised. The Flip recombinase gene was removed and the floxed line (FBXO7 fl/fl) bred to homozygosity. Since I used the FBXO7 $\mathrm{fl} / \mathrm{fl}$ mice to breed all conditional lines in this study, I initially controlled that the FBXO7 $\mathrm{fl} / \mathrm{fl}$ line itself did not show any motor abnormalities and therefore tested 12 months old FBXO7 fl/fl animals against wild type littermates. I performed; open field test for ambulation, rotarod and balance beam for coordination, pole test for dopaminergic neuron 
dysfunction and elevated plus maze for general anxiety (Figure 3.4.1). Only in the elevated plus maze there was a difference pointing to $\mathrm{FBXO} 7 \mathrm{fl} / \mathrm{fl}$ males being less anxious than their controls.
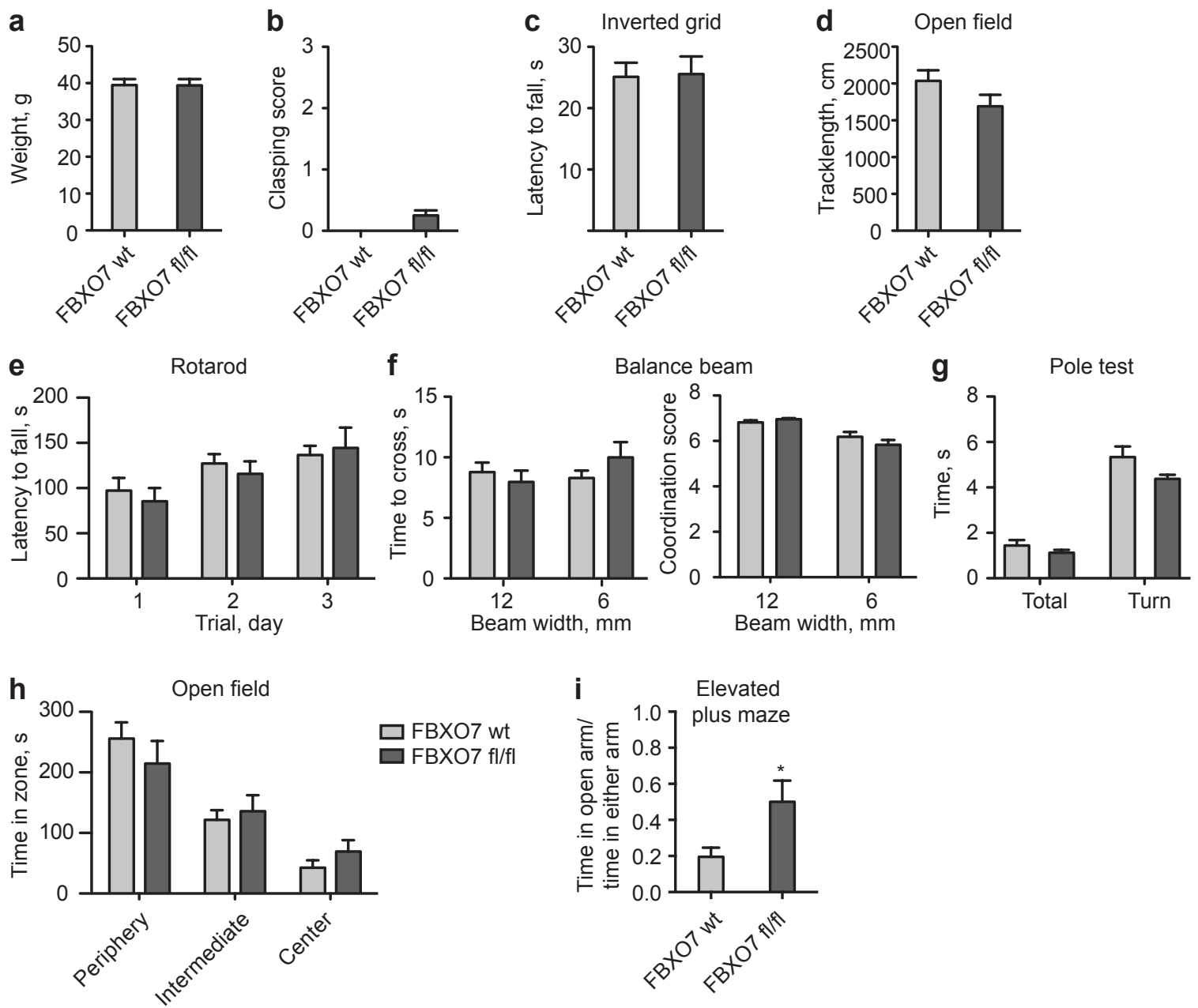

Figure 3.4.1 FBXO7 fl/fl show no abnormal behavioral phenotype. $\mathrm{FBXO} 7 \mathrm{fl} / \mathrm{fl}(\mathrm{N}=9)$ and wild type controls $(\mathrm{N}=8)$ of 12 months of age were subjected to behavioral tests as previously described. Animal weight (a). Tail suspension test (b). Inverted grid (c). Open field, track length (d). Rota-rod (e). Balance beam, time to cross and coordination score (f). Pole test (g). Open field, time spent in different zones (h). Elevated plus maze (i). Data represent mean \pm SEM (t-test, $\left.{ }^{*} p<0.05\right)$.

\subsubsection{FBXO7 protein expression is reduced in cortex of NEX-Cre;fl/fl mice}

The pyramidal symptoms seen in PARK15 patients indicate a disturbance in the upper motor neurons. We chose to model these symptoms by crossing our FBXO7 fl/fl line with a Cre deleter line under the control of the NEX promoter whose expression is strong in the excitatory neurons in the cortex, hippocampus and olfactory bulb (Goebbels et al., 2006). The NEX-Cre line was kept heterozygous at all times and bred to homozygous FBXO7 $\mathrm{fl} / \mathrm{fl}$ mice. The resulting double heterozygot animals were bred to $\mathrm{FBXO} 7 \mathrm{fl} / \mathrm{fl}$ mice. For 
behavioral and cellular analysis NEX-Cre+/-; FBXO7 fl/fl (NEX-Cre;fl/fl) were tested together with NEX-Cre+/-; FBXO7 wt (NEX-Cre) and NEXwt; FBXO7 fl/fl (FBXO7 fl/fl) as controls. Pups were genotyped at P18-23 (Figure 3.4.2a) and no embryonic lethality was found (Table 3.4.1).

Table 3.4.1 Loss of FBXO7 in NEX-Cre;fl/fl mice is not embryonically lethal. Pups from 20 litters were genotyped between the age of P15 and P25 and ratio of genotypes calculated.

\begin{tabular}{|c|c|c|c|c|c|c|c|c|c|c|}
\hline \multirow[b]{2}{*}{ Litter } & \multicolumn{5}{|c|}{ Male } & \multicolumn{5}{|c|}{ Female } \\
\hline & $\begin{array}{l}\text { NEX- } \\
\text { Cre;fl+ }\end{array}$ & $\begin{array}{l}\text { NEX- } \\
\text { Cre;fl/fl }\end{array}$ & $\begin{array}{c}\text { FBXO7 } \\
\mathrm{fl} /+\end{array}$ & $\begin{array}{c}\text { FBXO7 } \\
\text { fl/fl }\end{array}$ & Total & $\begin{array}{l}\text { NEX- } \\
\text { Cre;fl+ }\end{array}$ & $\begin{array}{c}\text { NEX- } \\
\text { Cre;fl/fl }\end{array}$ & $\begin{array}{c}\text { FBXO7 } \\
\text { fl/ }+\end{array}$ & $\begin{array}{c}\text { FBX07 } \\
\text { fl/fl }\end{array}$ & Total \\
\hline 1 & & 2 & 2 & 1 & 5 & 2 & & 1 & 1 & 4 \\
\hline 2 & 3 & & & 1 & 4 & & & & 1 & 1 \\
\hline 3 & & & & 3 & 3 & 1 & 1 & & & 2 \\
\hline 4 & & 2 & & & 2 & 1 & 1 & & 4 & 6 \\
\hline 5 & 2 & & 2 & 1 & 5 & & 1 & 1 & 1 & 3 \\
\hline 6 & 1 & & & & 1 & & & 1 & 2 & 3 \\
\hline 7 & & & & 1 & 1 & 1 & 1 & 1 & & 3 \\
\hline 8 & & 1 & & 1 & 2 & 1 & & & 2 & 3 \\
\hline 9 & 1 & 1 & 2 & 1 & 5 & 1 & & 1 & 2 & 4 \\
\hline 10 & & 1 & 1 & 2 & 4 & 1 & & 1 & & 2 \\
\hline 11 & & 1 & & 2 & 3 & & & & 2 & 2 \\
\hline 12 & & & 2 & & 2 & 1 & 2 & & & 3 \\
\hline 13 & 1 & & 1 & & 2 & & 1 & 2 & 2 & 5 \\
\hline 14 & 2 & 2 & 1 & 2 & 7 & & 1 & & & 1 \\
\hline 15 & & 1 & 1 & & 2 & & 1 & & 3 & 4 \\
\hline 16 & 2 & & & & 2 & 1 & 1 & & & 2 \\
\hline 17 & 2 & 2 & 1 & 2 & 7 & 2 & & 1 & & 3 \\
\hline 18 & 3 & 1 & 1 & 2 & 7 & 1 & & 1 & & 2 \\
\hline 19 & 2 & 3 & 1 & & 6 & & 2 & & 2 & 4 \\
\hline 20 & & 1 & 1 & 1 & 3 & 2 & 1 & 1 & 1 & 5 \\
\hline Sum & 19 & 18 & 16 & 20 & 73 & 15 & 13 & 11 & 23 & 62 \\
\hline Ratio & 0.260 & 0.247 & 0.219 & 0.274 & & 0.242 & 0.210 & 0.177 & 0.371 & \\
\hline
\end{tabular}

We verified the knockout of FBXO7 by means of qPCR and found FBXO7 mRNA to be reduced to about $70 \%$ in cortex and about $20 \%$ in the striatum (Figure $\mathbf{3 . 4 . 2 b}$ ). I then performed immunoblotting to determine the expression of FBXO7, and found that FBXO7 protein levels were also reduced in cortex but not in the cerebellum (Figure 3.4.2c). 

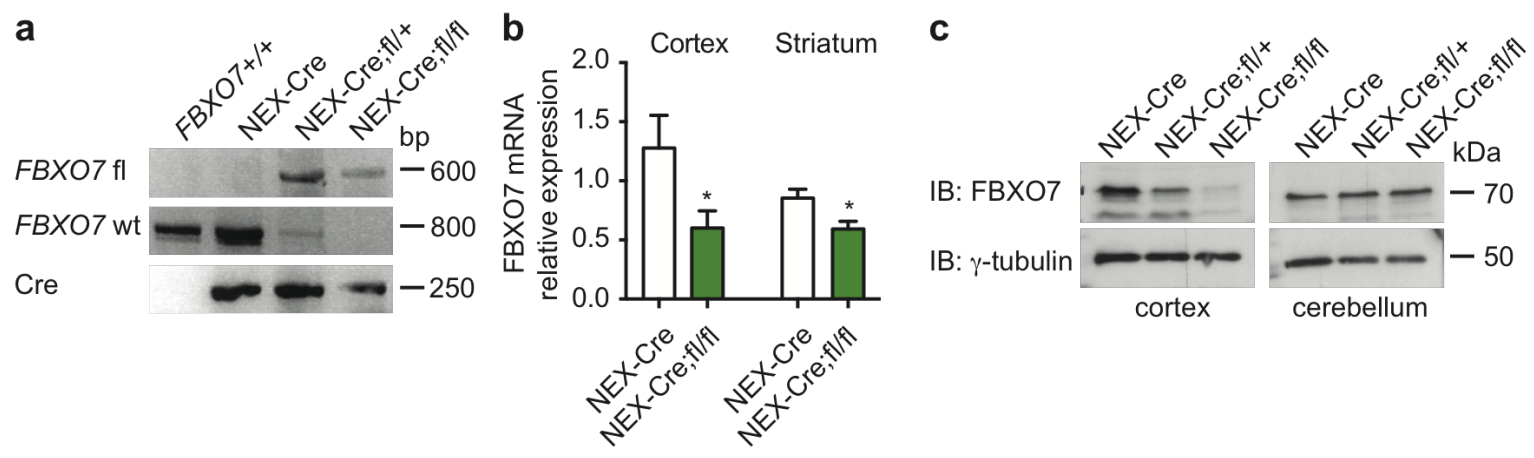

Figure 3.4.2 FBXO7 protein and mRNA expression is reduced in cortex of NEX-Cre;fl/fl mice. Tail biopsies from NEX-Cre;fl/fl mice and controls were lysed and subjected to PCR analysis with primers for FBXO7 wild type, loxp2 and Cre (a). cDNA was synthesized from cortex, and striatum of NEX-Cre $(\mathrm{N}=6)$ and NEX-Cre;fl/fl $(\mathrm{N}=6)$ mice and subjected to RT-PCR analysis with primers against FBXO7 (b). $\beta$-actin was used as reference sample. Data represent mean \pm SEM (MannWhitney non-parametric t-test, $\left.{ }^{*} p<0.05\right)$. Tissue from cortex and cerebellum of 2 months old NEXCre;fl/fl mice and controls was lysed and subjected to immunoblotting with antibodies directed against FBXO7 (c). $\gamma$-tubulin was used as loading control.

\subsubsection{NEX-Cre;fl/fl mice show spasticity and decreased coordination}

To characterize the motor phenotype of the NEX-Cre;fl/fl mice, I performed an array of behavioral tests. The weight of these animals was unchanged at 2 months of age, but they did not gain any weight in the following 2 months, which resulted in a significant difference in weight compared to controls at 4 months of age (Figure 3.4.3a). I initially noted a strong hind limb clasping at 2 months, which persisted until 4 months of age (Figure 3.4.3b,c). A personal observation to be noted is that the mice were hard to handle upon initial contact. They would spasm and bite down on the cage grid or initiate a spastic jumping if let lose. This behavior became less pronounced upon repeated handling. 

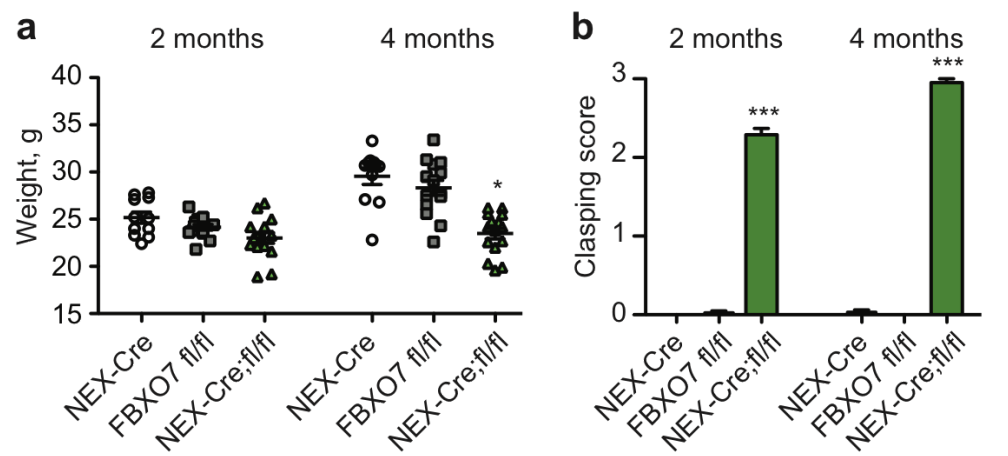

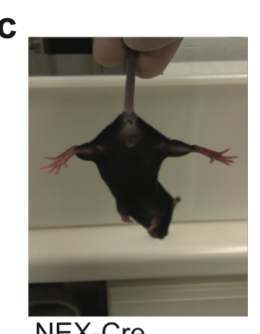

NEX-Cre

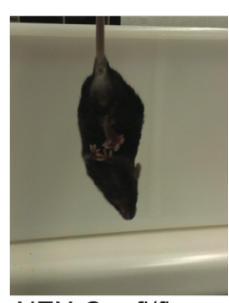

NEX-Cre;fl/fl

Figure 3.4.3 NEX-Cre;fl/fl show no increase in weight and a strong hind limb clasping up to 4 months of age. NEX-Cre (N=11), FBXO7 fl/fl $(\mathrm{N}=14)$ and $\mathrm{NEX}-\mathrm{Cre} ; \mathrm{fl} / \mathrm{fl}(\mathrm{N}=15)$ mice were weighed at 2 and 4 months (a). Data represent mean \pm SEM (ANOVA, Bonferronis Multiple Comparison test, $\left.{ }^{*} p<0.05\right)$. They were also subjected to the tail suspension test for $3 \times 10$ seconds and scored for hind limb clasping with 0 being normal and 3 given when both hind limbs were clasped together more than $50 \%$ of the time (b). Data represent mean \pm SEM (Kruskal-Wallis non-parametric ANOVA, Dunn's Multiple Comparison test, $\left.{ }^{* * *} p<0.001\right)$. Example picture of hind limb clasping in 2 months old mice (c).

To assess the motor control in the NEX-Cre;fl/fl mice, I subjected them to the balance beam and rotarod test (Figure 3.4.4). Both the time it took them to cross a balance beam of either 12 or $6 \mathrm{~mm}$ width, and the coordination score was measured (Figure 3.4.4a,b). Here, NEX-Cre;fl/fl mice required more time crossing the beam and showed lower coordination score than their control animals at 2 months. This reduction in motor control progressed even further at 4 months, where some mice were not able to cross the beam without falling down. In the rotarod test, I observed the same: The NEX-Cre;fl/fl mice managed to stay at the rotating rod significantly shorter than the controls and were clearly uncoordinated in their movements both at 2 months and even more so at 4 months of age (Figure 3.3.4c). 

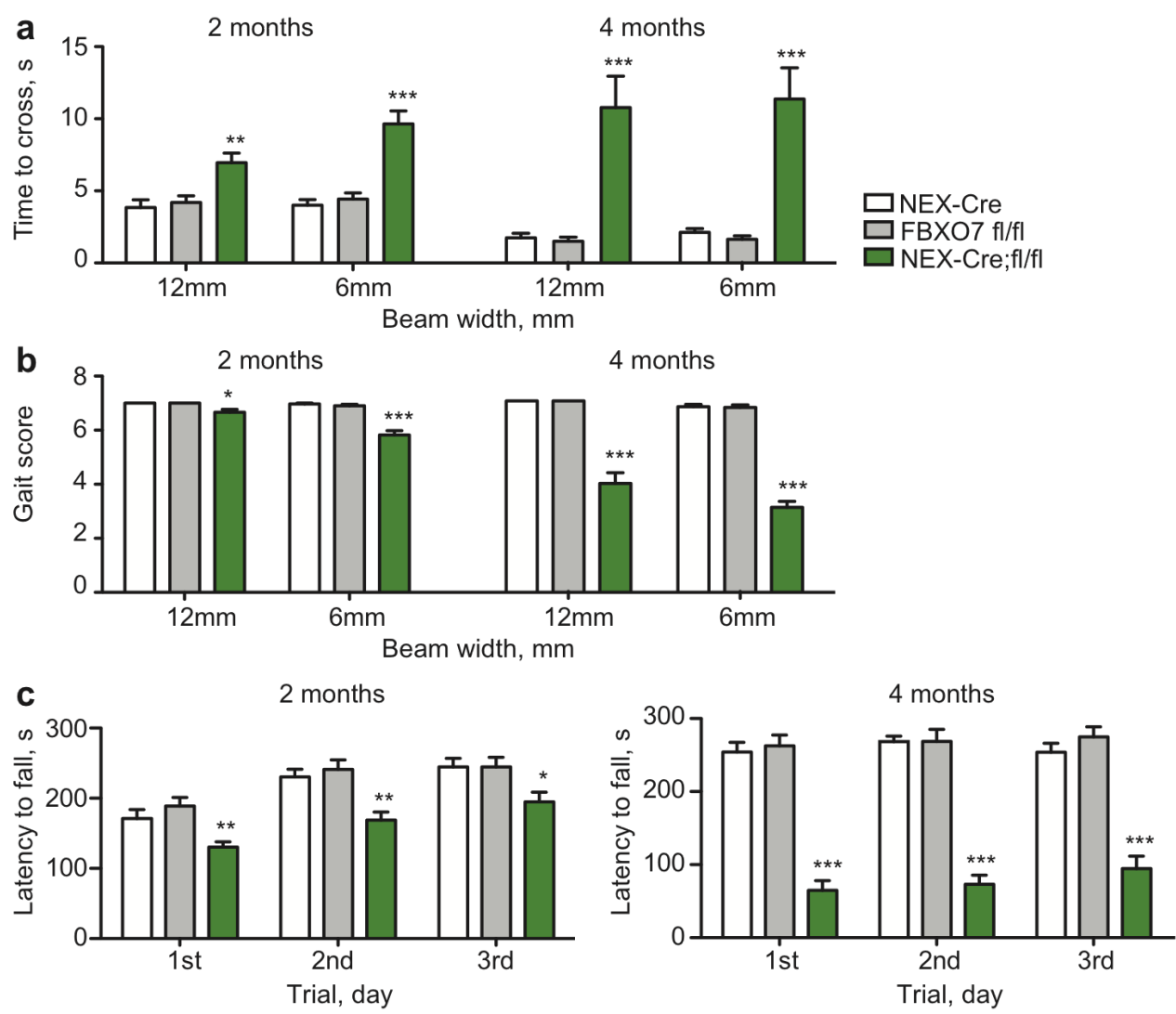

Figure 3.4.4 NEX-Cre;fl/fl mice show progressive decrease in motor coordination. NEX-Cre $(\mathrm{N}=11), \mathrm{FBXO} 7 \mathrm{fl} / \mathrm{fl}(\mathrm{N}=14)$ and $\mathrm{NEX}-\mathrm{Cre} ; \mathrm{fl} / \mathrm{fl}(\mathrm{N}=15)$ mice were subjected to the balance beam test at 2 and 4 months of age $(a, b)$. Time needed to cross the $80 \mathrm{~cm}$ long beam of two different widths ( 6 and $12 \mathrm{~mm}$ ) was recorded (a). Animals were scored according to performance with 0 being less than 2 slips and 7 being unable to cross beam (b). Data represent mean \pm SEM (Kruskal-Wallis non-parametric ANOVA, Dunn's Multiple Comparison test, $\left.{ }^{*} p<0.05,{ }^{* *} p<0.01,{ }^{* * *} p<0.001\right)$. The mice were also subjected to the rotarod test at 2 (c) and 4 months (d) with an accelerating protocol from 4-40 rpm in 5 minutes. Animals were tested on three consecutive days with 3 trials per day and 20 minutes intra-test interval and latency to fall measured. Data represent mean \pm SEM (ANOVA, Bonferronis Multiple Comparison test, ${ }^{*} p<0.05,{ }^{* *} p<0.01,{ }^{* * *} p<0.001$ ).

In these tests, I could show that the NEX-Cre;fl/fl mice display uncoordinated movements and progressive loss of motor control from 2 to 4 months of age. Hence, we conclude that loss of FBXO7 in excitatory neurons of the forebrain triggers motor defects in mice.

\subsubsection{NEX-Cre;fl/fl mice show hyperactivity and stereotypic behavior}

In the open field, the NEX-Cre;fl/fl mice moved significantly more than their controls (Figure 3.4.5a). They traveled about double the distance at 2 months of age and this hyperactivity persisted at 4 months despite the progressive loss of motor control. As the open field is a novel environment for the mice, I explored if the hyperactivity was consistent in a familiar environment. In collaboration with Dr. Ekrem Dere and Anja 
Ronnenberg from the Ehrenreich lab, we subjected the mice to the Laboratory Behavior Observation, Registration and Analysis System (LABORAS) and observed the same hyperactivity there (Figure 3.4.5b-e). The NEX-Cre;fl/fl mice traveled a longer distance, spent more time moving, less time immobile and moved at a higher velocity than their age-matched controls.

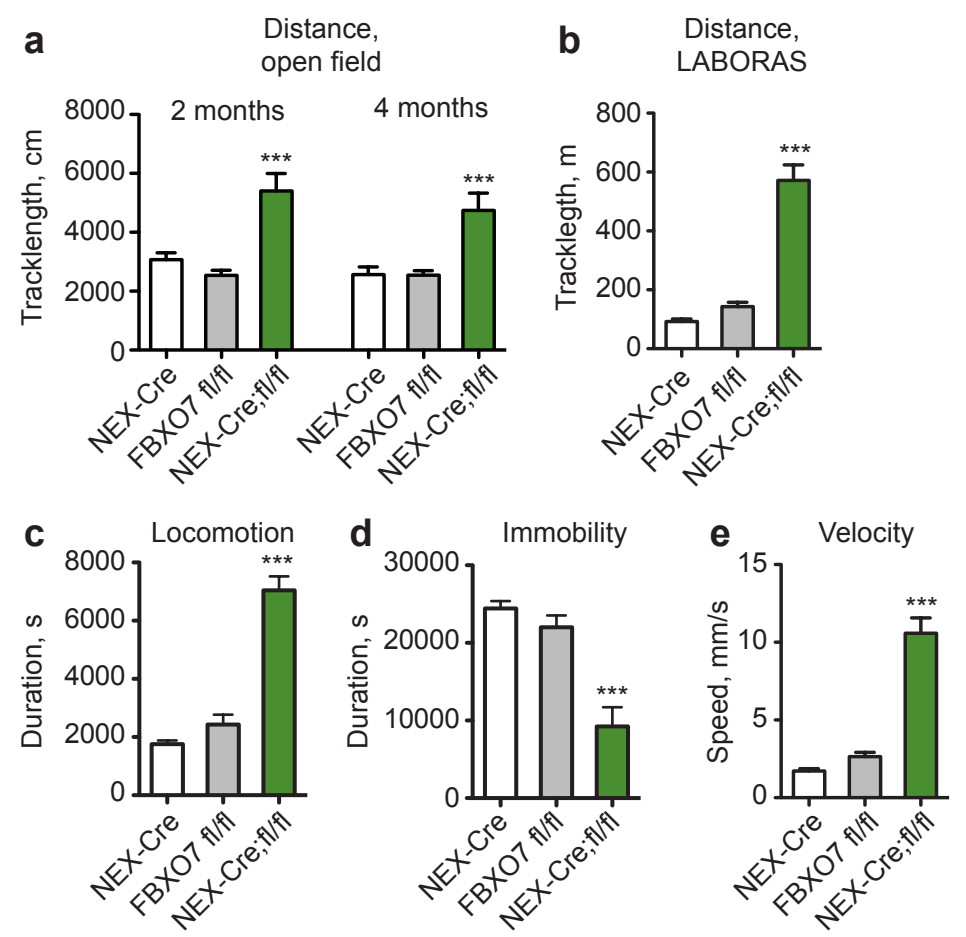

Figure 3.4.5 NEX-Cre;fl/fl mice show severe hyperactivity. NEX-Cre ( $\mathrm{N}=11), \mathrm{FBXO}$ fl/fl $(\mathrm{N}=14)$ and NEX-Cre;fl/fl $(\mathrm{N}=15)$ mice were subjected to the open field test at 2 and 4 months of age (a). The mice were placed in the middle of an open field of $60 \mathrm{~cm}$ diameter and total travel length was measured with the Viewer software after 7 minutes. NEX-Cre $(\mathrm{N}=5), \mathrm{FBXO} 7 \mathrm{fl} / \mathrm{fl}(\mathrm{N}=5)$ and NEXCre;fl/fl $(\mathrm{N}=6)$ mice were placed in the LABORAS homecage system over a time period of 15 hours. Path length (b), time spent in motion (c), time spent immobile (d) and velocity was recorded. Data represent mean \pm SEM (ANOVA, Bonferronis Multiple Comparison test, ${ }^{* * *} \mathrm{p}<0.001$ ). LABORAS experiment performed by Anja Ronnenberg and analyzed by Dr. Ekrem Dere.

Another striking feature in the open field test was that the NEX-Cre;fl/fl mice showed a high degree of stereotypic circling (Figure 3.4.6a). The circling was assessed on two separate occasions and not unidirectional for an individual animal, but consistent within a trial (Table 3.4.2). The number and diameter of the circles varied between animals as well as between trials.

Table 3.4.2 NEX-Cre;fl/fl mice stereotypic circling is uni-directional within test session. NEXCre;fl/fl mice and age-matched controls were subjected to the open field test twice with 1 week in between at 2 months of age. The mice were placed in the middle of an open field of $60 \mathrm{~cm}$ 
diameter and movement pattern recorded with the Viewer software for 7 minutes. Number of circles and direction was recorded in each trial.

\begin{tabular}{rrrrr} 
& \multicolumn{2}{c}{ Circles } & \multicolumn{2}{c}{ Direction } \\
Animal & Trial 1 & Trial 2 & Trial 1 & Trial 2 \\
\hline 2910 & 29 & $32+14$ & $\mathrm{R}$ & $\mathrm{R}+\mathrm{L}$ \\
2941 & 181 & $7+5$ & $\mathrm{~L}$ & $\mathrm{~L}+\mathrm{R}$ \\
2785 & 40 & 63 & $\mathrm{~L}$ & $\mathrm{~L}$ \\
2795 & 76 & 11 & $\mathrm{R}$ & $\mathrm{L}$ \\
2798 & 27 & 14 & $\mathrm{R}$ & $\mathrm{L}$ \\
2799 & 6 & 312 & $\mathrm{R}$ & $\mathrm{L}$ \\
2804 & 197 & 74 & $\mathrm{~L}$ & $\mathrm{R}$ \\
2806 & 17 & 22 & $\mathrm{R}$ & $\mathrm{R}$ \\
2950 & 69 & 50 & $\mathrm{R}$ & $\mathrm{L}$ \\
2952 & 15 & 10 & $\mathrm{R}$ & $\mathrm{L}$ \\
2953 & 30 & 7 & $\mathrm{~L}$ & $\mathrm{~L}$ \\
3029 & 29 & 19 & $\mathrm{~L}$ & $\mathrm{~L}$ \\
3030 & 20 & $14+6$ & $\mathrm{R}$ & $\mathrm{R}+\mathrm{L}$ \\
3109 & 9 & 10 & $\mathrm{R}$ & $\mathrm{R}$ \\
3110 & 125 & 20 & $\mathrm{R}$ & $\mathrm{L}$ \\
\hline
\end{tabular}

We again took advantage of the LABORAS home-cage system and saw the same circling pattern, meaning that the stereotypic circling is not stress-induced (Figure 3.5.6b). The circling direction changed here as well suggesting that potential lesions are not unilateral (Figure 3.4.6c).

a

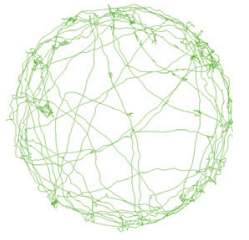

NEX-Cre

b
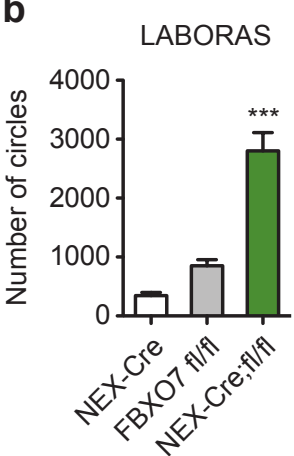

Open field

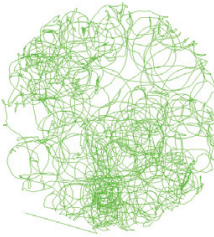

NEX-Cre;fl/fl

C

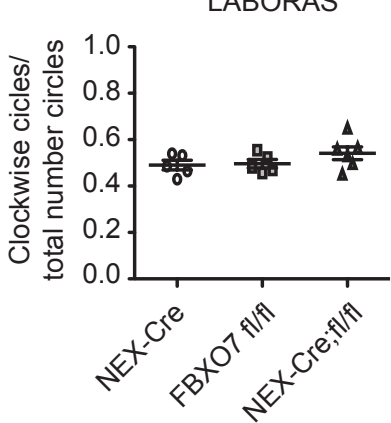

Figure 3.4.6 NEX-Cre;fl/fl mice show stereotypic circling. 2 months old NEX-Cre and NEX-Cre;fl/fl mice were placed in the middle of an open field of $60 \mathrm{~cm}$ diameter and movement pattern recorded with the Viewer software for 7 minutes. Examples of NEX-Cre movement pattern (left panel) and stereotypic circling by NEX-Cre;fl/fl in small diameter (middle panel) and large diameter (right panel) is shown (a). NEX-Cre $(\mathrm{N}=5)$, FBXO7 fl/fl $(\mathrm{N}=5)$ and NEX-Cre;fl/fl $(\mathrm{N}=6)$ mice were placed in the LABORAS homecage system over a time period of 15 hours. Number of circles made in the 
LABORAS homecage system was measured over a time period of 15 hours (b). Ratio of clockwise versus counter clockwise circles for individual animals is shown (c). Data represent mean \pm SEM (ANOVA, Bonferronis Multiple Comparison test, ${ }^{* * *} \mathrm{p}<0.001$ ). LABORAS experiment performed by Anja Ronnenberg and analyzed by Dr. Ekrem Dere.

Many types of stereotypic behaviors are found in rodents, so $\mathrm{Dr}$. Dere also analyzed rearing, grooming, scratching and climbing behavior (Figure 3.4.6). Scratching and rearing frequency were significantly increased for the NEX-Cre;fl/fl mice, but they did not groom more than controls (Figure 3.4.6a-c). The climbing behavior was surprisingly significantly different between the control groups, which is hard to explain with the current data and should be repeated with a larger animal number (Figure 3.4.7d).
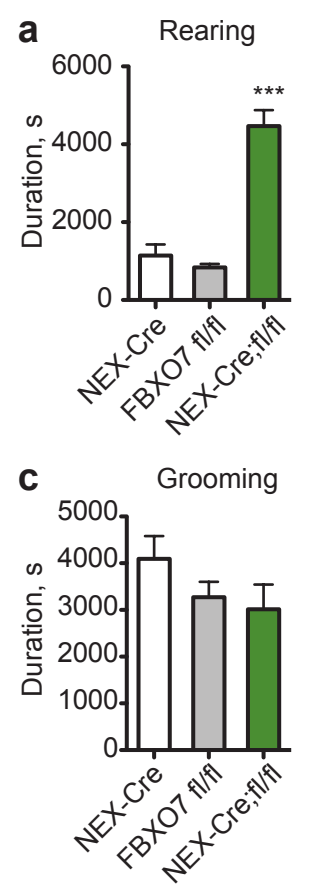
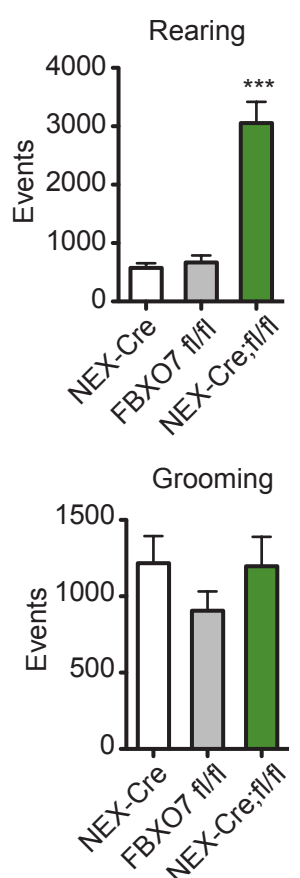
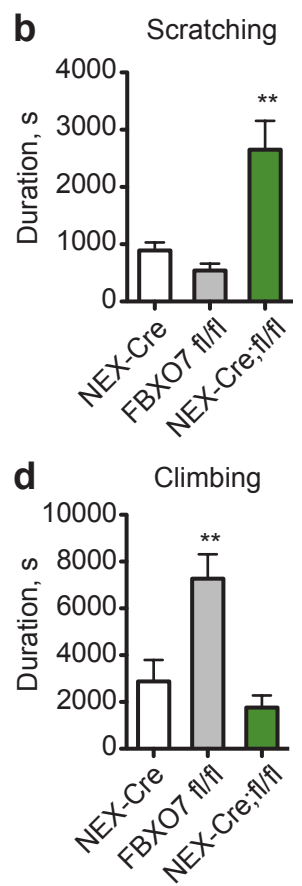
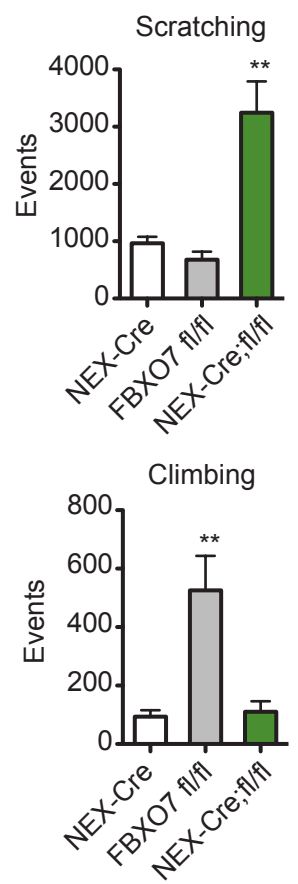

Figure 3.4.7 NEX-Cre;fl/fl mice show increased rearing and scratching, but no difference in grooming and climbing. NEX-Cre $(\mathrm{N}=5), \mathrm{FBXO7} \mathrm{fl} / \mathrm{fl}(\mathrm{N}=5)$ and NEX-Cre;fl/fl $(\mathrm{N}=6)$ mice were placed in the LABORAS homecage system over a time period of 15 hours. Duration and frequency of rearing (a), scratching (b), grooming (c) and climbing (d) was measured. Data represent mean \pm SEM (ANOVA, Bonferronis Multiple Comparison test, $\left.{ }^{* *} p<0.01,{ }^{* * *} p<0.001\right)$. LABORAS experiment performed by Anja Ronnenberg and analyzed by Dr. Ekrem Dere.

Another sign of stereotypic behavior in rodents is increased marble burying. I therefore presented the mice to $4 \times 6$ marbles laid out on soft bedding. Normal mice try to bury the marbles in the bedding. Obsessive marble burying or moving are considered signs of repetitive or stereotypic behavior. In the NEX-Cre;fl/fl mice however, I saw a reduction in both number of marbles buried and number of marbles moved (Figure 3.4.8). 

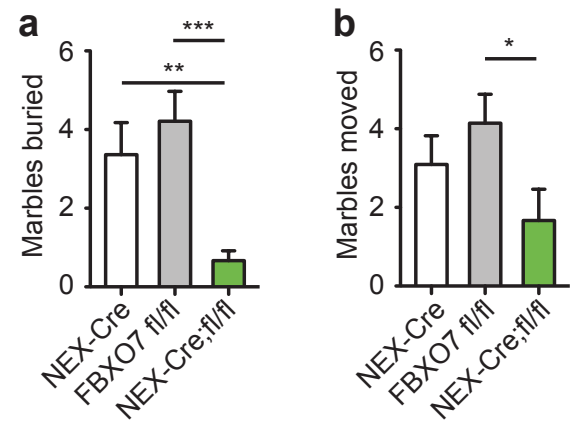

Figure 3.4.8 NEX-Cre;fl/fl mice show decreased marble moving and burying. NEX-Cre $(\mathrm{N}=11), \mathrm{FBXO} 7 \mathrm{fl} / \mathrm{fl}$ $(\mathrm{N}=14)$ and NEX-Cre;fl/fl $(\mathrm{N}=15)$ mice were placed in a cage $30 \times 45 \mathrm{~cm}$ with $4 \times 6$ marbles placed out on top of soft bedding for 30 minutes. Number of marbles buried (a) and moved (b) was counted. Data represent mean \pm SEM (Kruskal-Wallis non-parametric ANOVA, Dunn's Multiple Comparison test, $\left.{ }^{*} p<0.05,{ }^{* *} p<0.01,{ }^{* * *} p<0.001\right)$.

Since the NEX promoter is also expressed in the hippocampus, I investigated if memory formation was affected in these mice. However, the severe motor deficits interfered with most standard memory tests. I therefore decided to test the animals for disturbances in spatial memory via the Y-maze test, where the mice are allowed to freely explore a maze consisting of three equal arms. A triplet is counted when all three arms are consecutively visited without re-entrance to an already visited arm. NEX-Cre;fl/fl mice entered the arms more often than controls (Figure 3.4.9a), but made equally many triplets (Figure 3.4.9b).
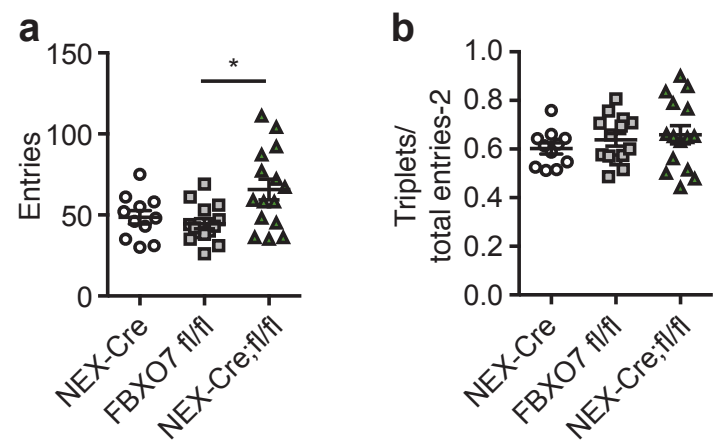

Figure 3.4.9 NEX-Cre;fl/fl mice show no aberrations in spatial memory, but patterned behavior in $\mathrm{Y}$-maze. NEX-Cre $(\mathrm{N}=11)$, FBXO7 $\mathrm{fl} / \mathrm{fl}(\mathrm{N}=14)$ and $\mathrm{NEX}-\mathrm{Cre} ; \mathrm{fl} / \mathrm{fl}(\mathrm{N}=15)$ mice were placed in a Y-maze with $15 \times 30 \mathrm{~cm}$ walled arms and recorded for 10 minutes. Total number of arm entries (a) and ratio of triplets (consecutive visits of all three arms) to total number of triplets possible (b) were counted. Data represent mean \pm SEM (ANOVA, Bonferronis Multiple Comparison test).

Some NEX-Cre;fl/fl animals repeated certain patterns, either duplicates or triplicates:

NEX-Cre;fl/fl 2795: CBABACB AC AC AC AC BACBA CB СB СB CB АСBACBCBABCBCB AC AC AC ВАСA СВ СВ СВ АСВСВАВАСАВ

NEX-Cre;fl/fl 3110: C ACB ACB ACB ACB ACB ACB АCB ACB АCB ACB ACB ACB ACB ACB ACB ACB ACB ACB CB CBA CBA CBA CBA CBA CBA CBA CBA CBA CBA CBA CBA C

This lead to a larger spread in triplicate ratios for the NEX;fl/fl animals (Figure 3.4.9b) and again shows a high degree of repetitive behavior, which does not only pertain to circling, but also arm entries in a mace. This repetitive pattern was also observed in a novel object recognition test (Figure 3.4.10). Here, the NEX-Cre;fl/fl mice would sniff one object for a couple of seconds, move to the other and sniff at this for a couple of seconds and then circle back to the first. This behavior continued for as long as the animal stayed 
uninterrupted and made the test impossible to perform, but again reflected a highly repetitive movement pattern.

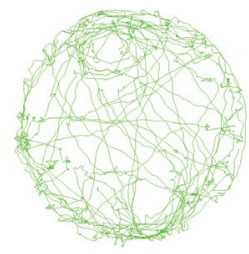

NEX-Cre

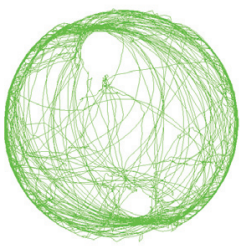

NEX-Cre;fl/fl

Figure 3.4.10 NEX-Cre;fl/fl mice show stereotypic movement pattern. 2 months old NEX-Cre and NEX-Cre;fl/fl mice were placed in the middle of an open field of $60 \mathrm{~cm}$ diameter with two different objects of similar size. Examples of NEX-Cre (left panel) and NEX-Cre;fl/fl movement pattern (right panel) is shown.

As the NEX-Cre;fl/fl mice did not gain weight from 2 to 4 months, we examined their feeding behavior. In the LABORAS home-cage system Dr. Dere found an increase in eating and a decrease in drinking duration compared to controls (Figure 3.4.11).

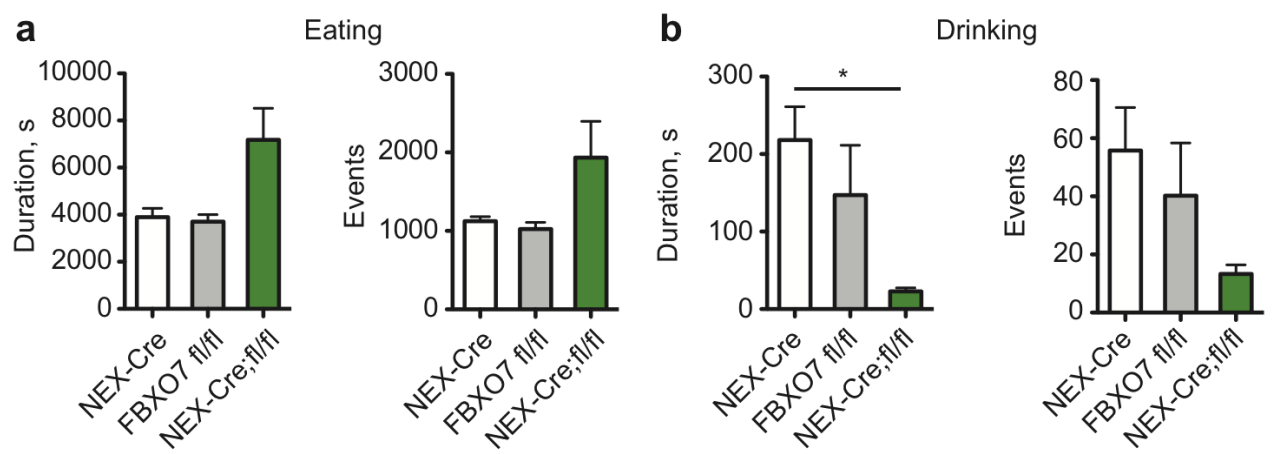

Figure 3.4.11 NEX-Cre;fl/fl mice show increased eating and reduced drinking frequency. NEX-Cre $(\mathrm{N}=5), \mathrm{FBXO} 7 \mathrm{fl} / \mathrm{fl}(\mathrm{N}=5)$ and NEX-Cre;fl/fl $(\mathrm{N}=6)$ mice were placed in the LABORAS homecage system over a time period of 15 hours. Eating (a) and drinking (b) events and duration was measured. Data represent mean \pm SEM (ANOVA, Bonferronis Multiple Comparison test, $\left.{ }^{*} \mathrm{p}<0.05\right)$. LABORAS experiment performed by Anja Ronnenberg and analyzed by Dr. Ekrem Dere.

Behavioral disturbances were found in many of the PARK15 patients, and one patient presented with panic attacks (Lohmann et al., 2015). I therefore conducted experiments to look into the anxiety state in the NEX-Cre;fl/fl mice. I first analyzed the time spend in different zones of the open field, but found no difference (Figure 3.4.12a). This was not surprising as the mice showed a high frequency of stereotypic circling. I then did an elevated plus maze test, which takes advantage of the rodent's tendency to seek shelter. A normal mouse will spend more time in one of the sheltered arms of the maze compared to the open arms. Here, the NEX-Cre;fl/fl mice seemed to be significantly less anxious than their controls and spend more than half their time in the open arms of the maze (Figure 3.4.12b). 
a

Open field
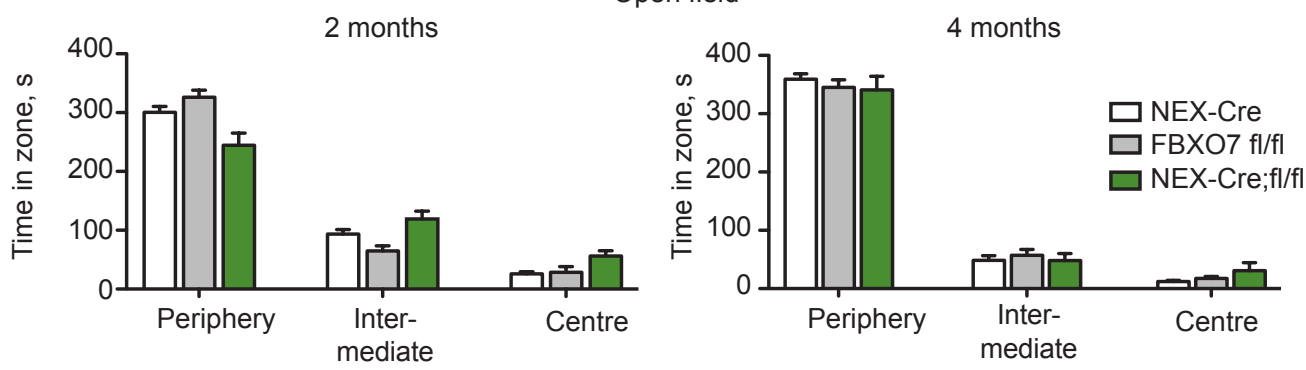

b

Elevated plus maze
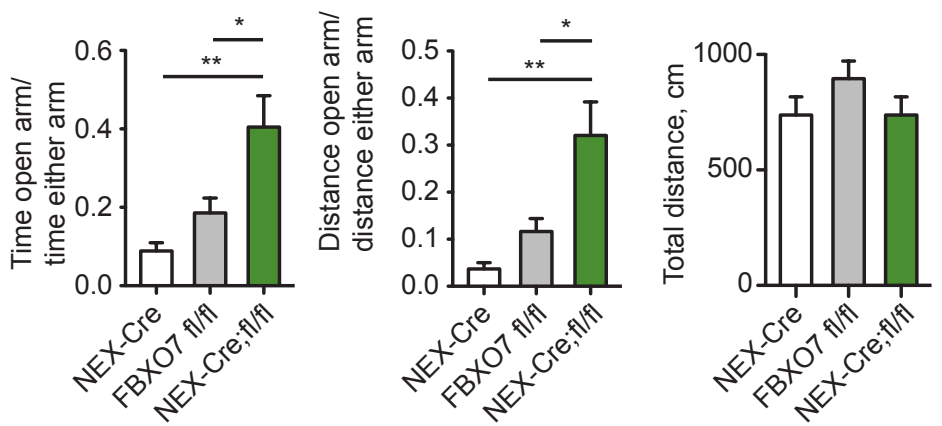

Figure 3.4.12 NEX-Cre;fl/fl mice show decreased anxiety in the elevated plus maze test. NEX-Cre (N=11), FBXO7 fl/fl $(\mathrm{N}=14)$ and NEX-Cre;fl/fl $(\mathrm{N}=15)$ mice were placed in the center of an open field $60 \mathrm{~cm}$ in diameter, and allowed to move freely for 7 minutes. Time spent in different zones was recorded (a). The same mice were placed in an Elevated Plus Maze with two open and two walled arms and allowed to move freely for 5 minutes and their movement pattern recorded by the Viewer software. Ratio of time spent in open arms versus all arms, ratio of distance traveled in open arm versus all arms and total path length was measured (b). Data represent mean \pm SEM (ANOVA, Bonferronis Multiple Comparison test, ${ }^{*} p<0.05,{ }^{* *} p<0.01$ ).

To confirm this finding, I subjected the mice to a light/dark paradigm. I placed the mice in the light with the option to escape into a dark compartment and measured the latency to escape and the time spent in each compartment (Figure 3.4.13). To my surprise, this test did not show that the NEX-Cre;fl/fl mice stayed longer in the light, but they showed increased latency to enter the dark compartment. The mice also crossed between the compartments significantly less than the controls. 

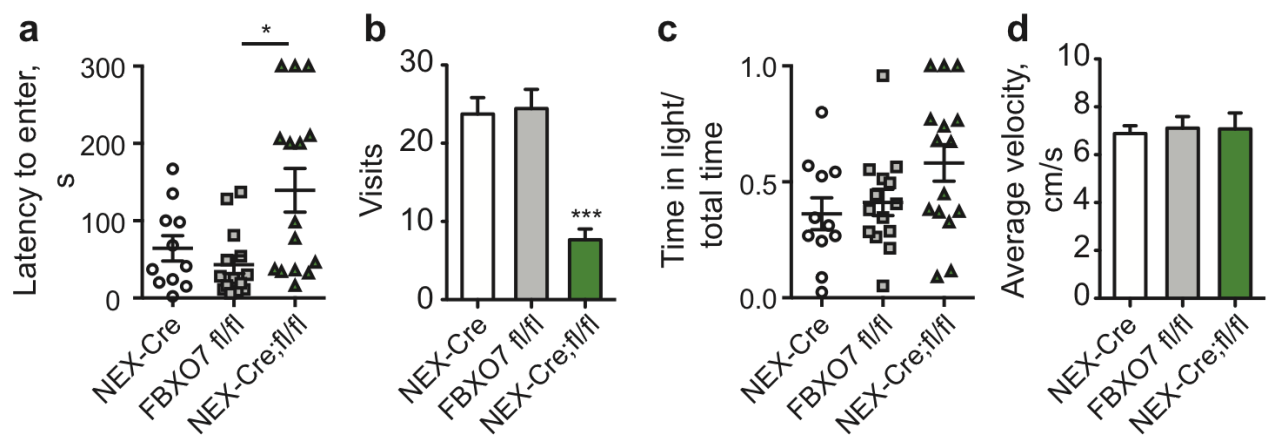

Figure 3.4.13 NEX-Cre;fl/fl mice show reduced change of location in light/dark paradigm. NEX-Cre $(\mathrm{N}=11), F B X O 7 \mathrm{fl} / \mathrm{fl}(\mathrm{N}=14)$ and NEX-Cre;fl/fl $(\mathrm{N}=15)$ mice were placed in the light compartment, $30 \times 15 \mathrm{~cm}$ of a Plexiglas box and their movement pattern recorded with the viewer software. Latency to enter dark compartment (a), $20 \times 30 \mathrm{~cm}$, number of visits to the dark side (b), ratio of time spent in light compartment to total time (c), and velocity of movement in light compartment (d) was measured. Data represent mean \pm SEM (ANOVA, Bonferronis Multiple Comparison test, $\left.{ }^{*} p<0.05,{ }^{* * *} p<0.001\right)$.

Since loss of smell can be a factor influencing other behavioral tests, I also conducted an olfactory test on the NEX-Cre conditional mouse line (Figure 3.4.14). Here, I could show that the NEX-Cre;fl/fl mice were divided into two groups. Either they found the treat as fast as control mice, or they did not recover the treat at all within the test time.

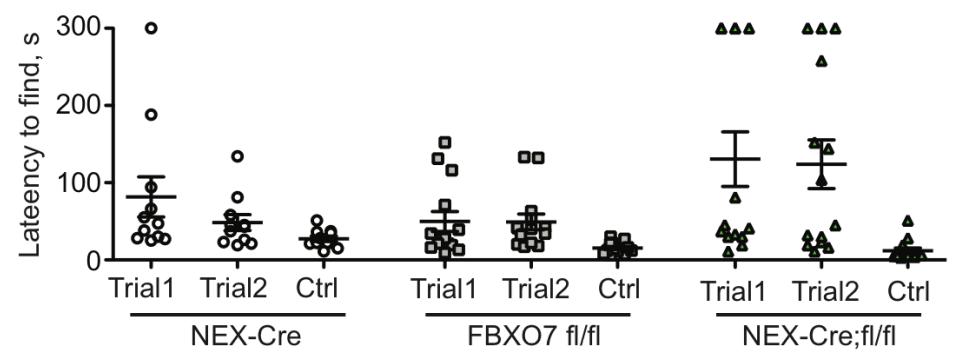

Figure 3.4.14 NEX-Cre;fl/fl mice show normal olfaction. NEX-Cre ( $\mathrm{N}=11)$, FBXO7 fl/fl $(\mathrm{N}=14)$ and NEX-Cre;fl/fl $(\mathrm{N}=15)$ mice were placed in a $30 \times 40$ container with a treat buried underneath the bedding on two different trials with different locations. On control trial the treat was placed on top of bedding in the middle of the cage. Data represent mean \pm SEM (Kruskal-Wallis non-parametric ANOVA, Dunn's Multiple Comparison test).

When these tests are compared a pattern emerges that suggest NEX-Cre;fl/fl mice to show perseverative locomotor behavior. These mice seem to have problems changing between behaviors and this is reflected in stereotypic, repetitive movements.

\subsubsection{NEX-Cre;fl/fl mice show altered protein expression in the striatum}

In the conventional FBXO7 knockout mouse line, David Brockelt found increased apoptosis in the cortex. I therefore investigated whether the total number of neurons in the cortex of the NEX-Cre;fl/fl mice differed from those of controls. So I performed 
immunohistochemistry with an antibody against the neuronal marker NeuN. I first quantified the thickness of the cortex and the ratio between the different cortical layers, but found no difference between control and NEX-Cre;fl/fl cortices (Figure 3.4.15a-c). I then counted the number of neurons in layer $V$ of the motor cortex (Figure 3.4.15d,e). Again, there was no significant difference between the NEX-Cre;fl/fl mice and the controls.

a

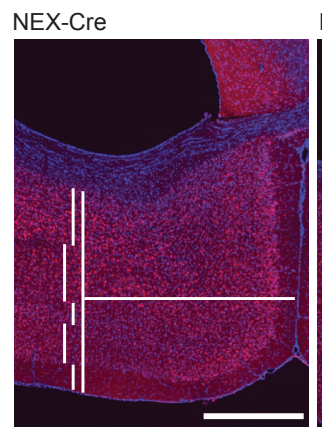

d

NEX-Cre

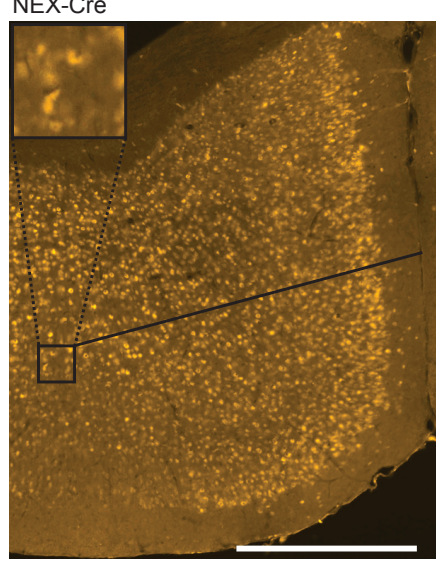

NEX-Cre;fl/fl

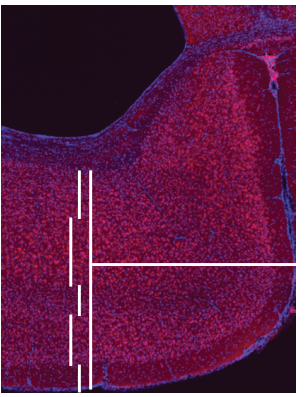

NEX-Cre;fl/fl
C

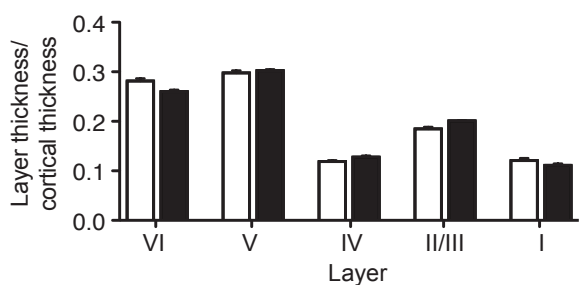

$\mathrm{fl} / \mathrm{fl}$

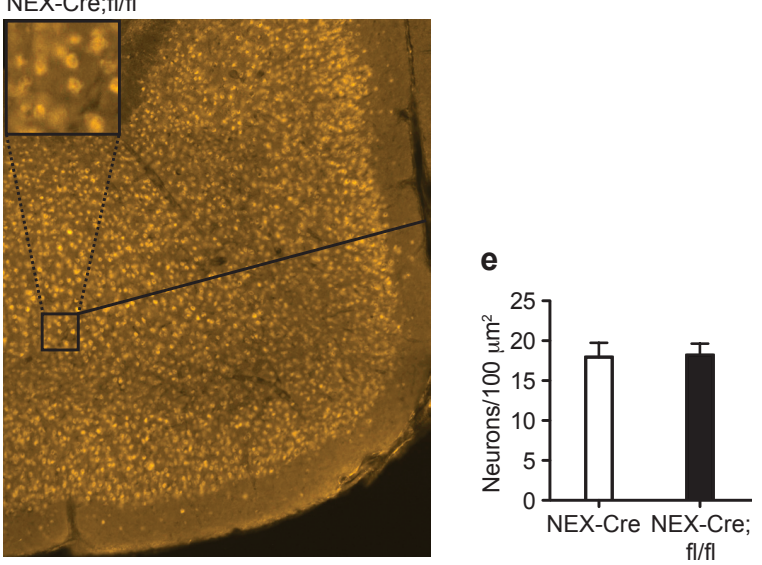

Figure 3.4.15 Cortical thickness and number of neurons in layer $\mathbf{V}$ seems normal in NEXCre;fl/fl mice. NEX-Cre;fl/fl $(\mathrm{N}=3)$ and NEX-Cre $(\mathrm{N}=3)$ mice were transcardially perfused and 30 $\mu \mathrm{m}$ brain sections cut with a cryostat. Sections were subjected to immunohistochemistry with an antibody directed against NeuN (red/yellow; a,d) and DAPI (blue), a nuclear dye (a). Images were measured with the Image J software and manually counted in a blinded manner. Cortical thickness (b), relative layer thickness (c) and number of neurons $/ 100 \mu \mathrm{m}^{2}$ (e) were quantified. Data represent mean \pm SEM (t-test). Scale bars equal $500 \mu \mathrm{m}$.

Since the NEX promoter is expressed mainly in excitatory neurons, I analyzed how a marker of glutamatergic neurons was affected in different brain areas. VGLUT1 is one of the main glutamatergic transporters and I examined its expression in cortex, cerebellum and striatum (Figure 3.4.16a, b, c). Consistent with the notion that there was no gross neuronal loss in the cortex, I also found no difference in VGLUT1 expression in the cortex between the genotypes. The cerebellum did also not seem to show a difference in VGLUT1 expression. However, in the striatum I saw a marked trend of decreased VGLUT1 expression (Figure 3.4.16c). 

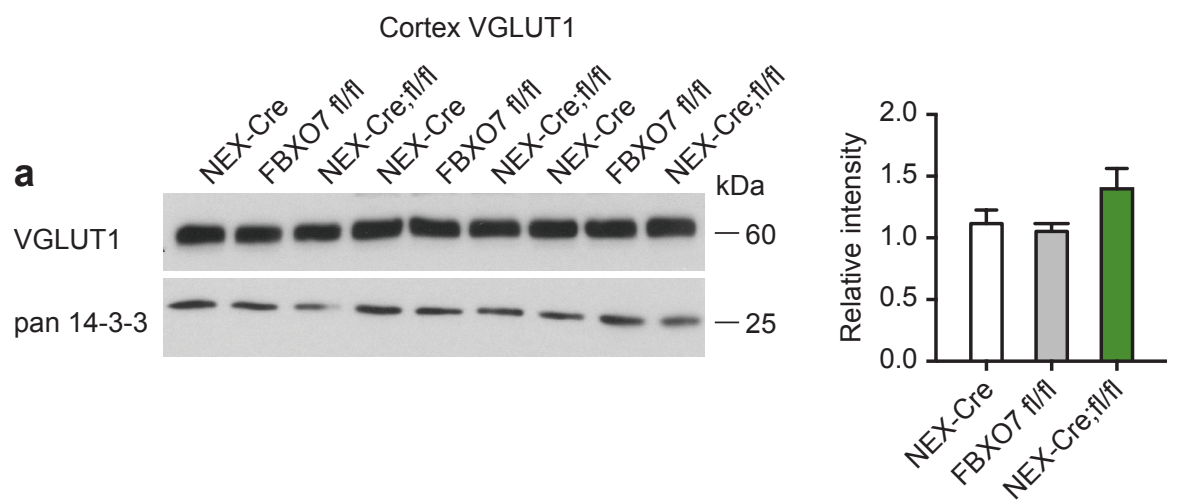

Cerebellum VGLUT1
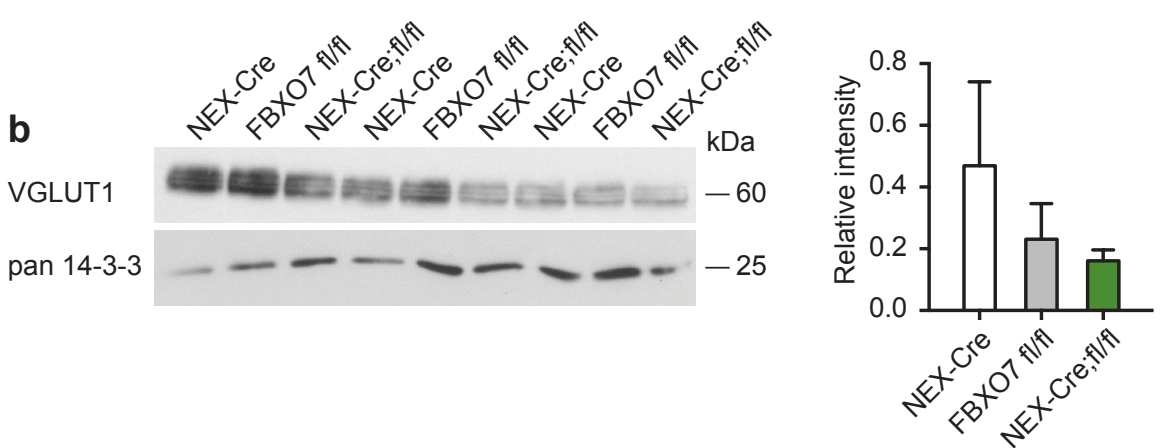

Striatum VGLUT1
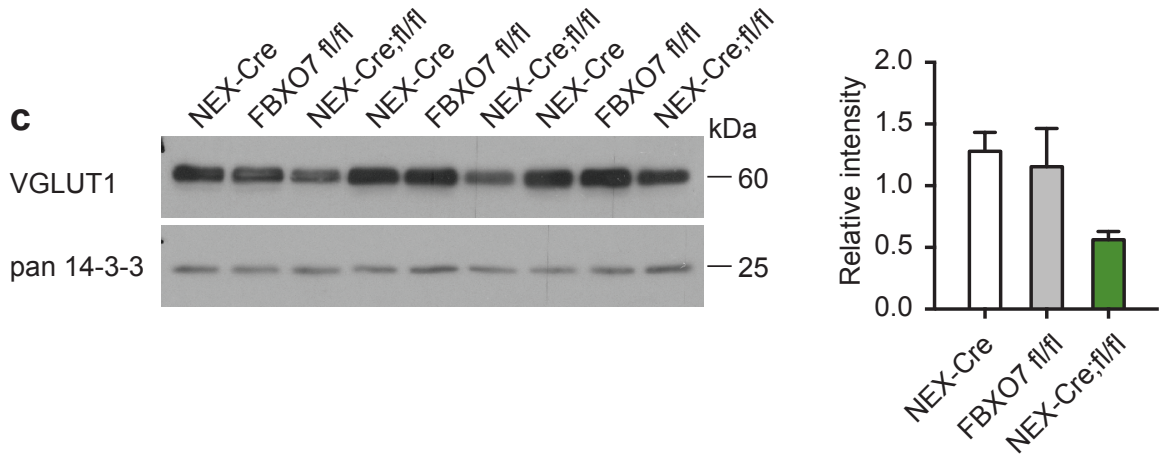

Figure 3.4.16 NEX-Cre;fl/fl animals show a decrease in VGLUT1 expression in the striatum. Cortical (a), cerebellar (b) and striatal (c) tissue from NEX-Cre;fl/fl and control mice was lysed and subjected to immunoblotting analysis with an antibody against VGLUT1. Blots were quantified with the ImageJ software and intensity normalized to loading control (pan 14-3-3). Values are expressed relative to first NEX-Cre sample. Data represent mean \pm SEM (ANOVA, Bonferronis Multiple Comparison test).

The striatum is a brain area that integrates input from different areas to regulate motor behavior. This integration depends heavily upon dopaminergic neurons. To determine if dopaminergic neurons were affected by the down-regulation of VGLUT1, I looked at DAT and $\mathrm{TH}$, two important proteins in dopaminergic regulation. By immunoblotting analysis I found DAT to be significantly up-regulated in the striatum of NEX-Cre,fl/fl mice (Figure 3.4.17). 


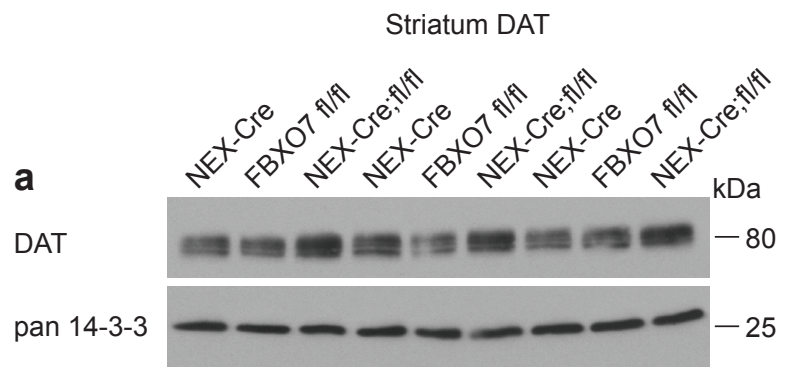

Striatum $\mathrm{TH}$

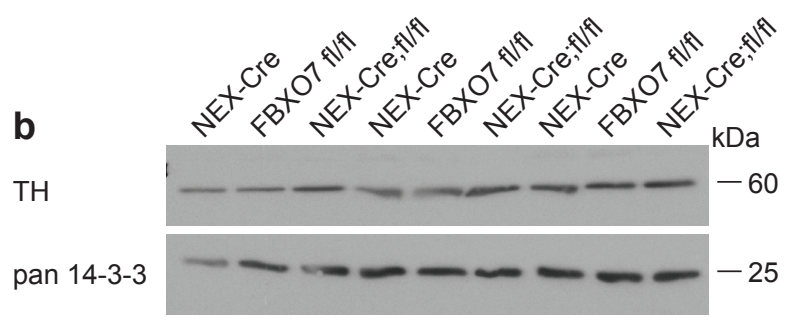

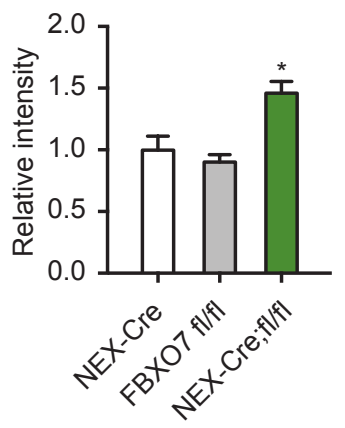

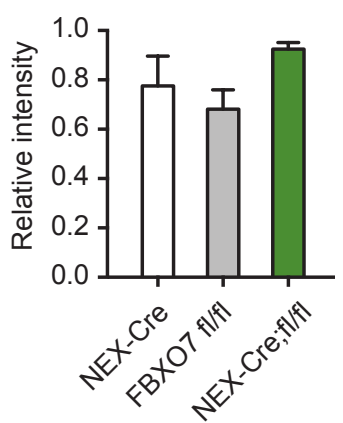

Figure 3.4.17 NEX-Cre;fl/fl mice show an increase in DAT expression in the striatum. Striatal tissue from NEX-Cre;fl/fl and control mice was lysed and subjected to immunoblotting analysis with antibodies against DAT (a) and TH (b). Blots were quantified with the ImageJ software and intensity normalized to loading control (pan 14-3-3). Values are expressed relative to first NEX-Cre sample. Data represent mean \pm SEM (ANOVA, Bonferronis Multiple Comparison test, ${ }^{*} p<0.05$ ).

In cooperation with Dr. Lars Tatenhorst I conducted HPLC analysis of dopamine and metabolite levels in the striatum of 2 months old NEX-Cre conditional knockout mice. Here, we could show that extracellular dopamine was significantly upregulated in the striatum of NEX-Cre;fl/fl mice (Figure 3.4.18).
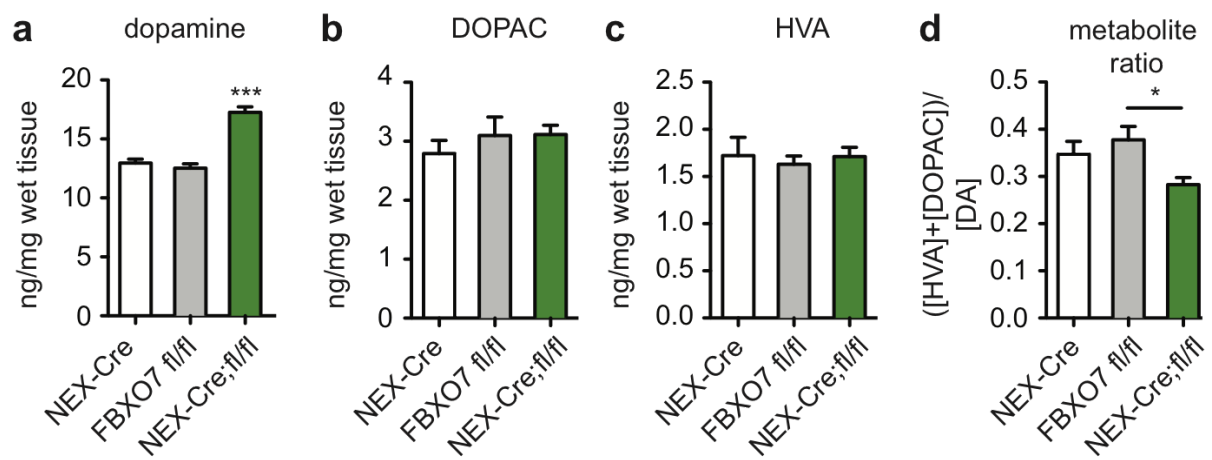

Figure 3.4.18 NEX-Cre;fl/fl mice show an increase of dopamine in the striatum. Striatal tissue from NEX-Cre $(\mathrm{N}=5)$, FBXO7 fl/fl $(\mathrm{N}=6)$ and NEX-Cre;fl/fl $(\mathrm{N}=10)$ mice was subjected to HPLC analysis for dopamine (a), DOPAC (b) and HVA (c). Metabolism is shown as ratio of HVA and DOPAC to dopamine (d). NEX-Cre $N=5$, FBXO7 fl/fl N=6, NEX-Cre;fl/fl $N=10$. Data represent 
mean \pm SEM (ANOVA, Bonferronis Multiple Comparison test, ${ }^{*} p<0.05,{ }^{* * *} p<0.001$ ). HPLC measurements conducted by Dr. Lars Tatenhorst.

The tight interplay I saw between FBXO7 and PI31 in the FBXO7 full knockout mouse model was further confirmed in the NEX-Cre mice, where I observed a significant downregulation of PI31 in the cortex of NEX-Cre;fl/fl mice compared to control (Figure 3.4.19).

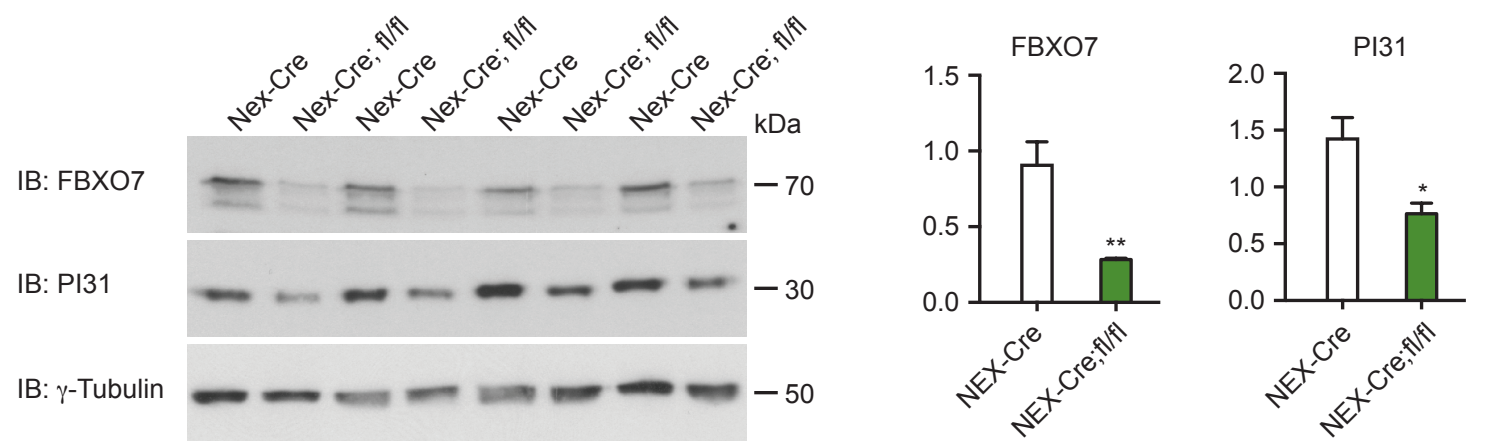

Figure 3.4.19 NEX-Cre;fl/fl mice show a decrease in PI31 levels. Cortical tissue lysates from NEX-Cre;fl/fl $(\mathrm{N}=4)$ and NEX-Cre $(\mathrm{N}=4)$ mice were subjected to immunoblotting with an anti-PI31 antibody. FBXO7 served as control for FBXO7 knockout and $\gamma$-tubulin as loading control. Data represent mean \pm SEM (t-test, $\left.{ }^{*} p<0.05,{ }^{* *} p<0.01\right)$.

In the conventional FBXO7 knockout mouse, I could not find a difference in oxidized protein levels (Figure 3.3.19), but these mice were analyzed only at P18. I therefore wanted to see if a longer exposure to $\mathrm{FBXO7}$ depletion caused protein oxidation. I analyzed the NEX-Cre;fl/fl mice at 2 months of age, but also here I found no indication of increased protein oxidation (Figure 3.4.20).

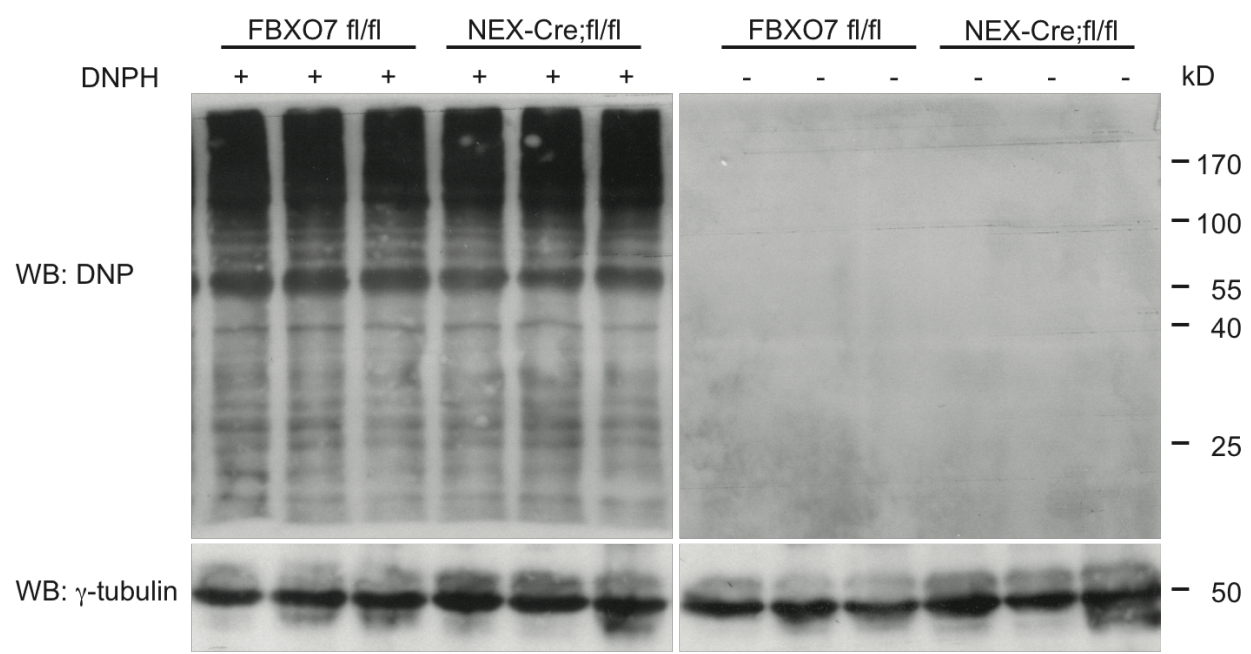

Figure 3.4.20 NEX-Cre;fl/fl mice show no difference in oxidized protein levels in cortex. Cortical tissue from NEX-Cre;fl/fl and control mice was lysed and exposed to 2,4dinitrophenylhydrazine (DNPH)(left panel) or control (right panel). The resulting derivatization of carbonyl groups in the protein side chains to 2,4-dinitrophenylhydrazone (DNP-hydrazone) was detected by an anti-DNP antibody. $\gamma$-tubulin served as loading control. 
The hyperactivity and stereotypic movement pattern seen in the behavioral experiments are consistent with a heightened dopaminergic tone in the striatum. Therefore, the increased level of dopamine in the striatum and the corresponding up-regulation of DAT in the NEX-Cre;fl/fl mice I show here could underlie the behavioral phenotype of these mice. In addition I show a decrease in VGLUT1 expression in striatum of NEX-Cre;fl/fl mice pointing to pyramidal neuron dysfunction.

\subsection{TH-Cre;fl/fl mice show progressive motor decline and loss of dopamine in the striatum}

PARK15 patients show Parkinsonian symptoms including bradykinesia and rigidity in addition to a response to L-dopa treatment (Table A1). In our conventional mouse model, we did not see any difference in dopamine levels and the animals died too young to notice if there was a progressive decline in motor function. We therefore set out to model the Parkinsonian phenotype of PARK15 patients by conditionally knocking out FBXO7 in catecholaminergic neurons. The previously described FBXO7 fl/fl mice were bred with a mouse line, where Cre recombinase expression is controlled by the tyrosine hydroxylase (TH) promoter (Savitt et al., 2005). The TH-Cre line was kept heterozygous and mice bred to homozygosity for FBXO7 fl/fl. Mice were genotyped for the presence of the loxp2 allele, the Cre gene and wild type FBXO7 to confirm correct breeding (Figure 3.5.1).

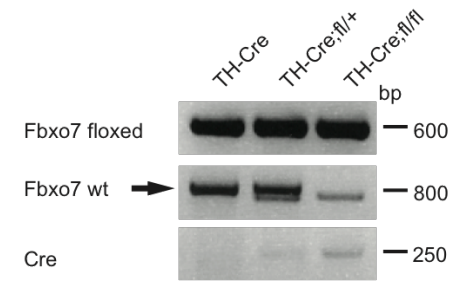

Figure 3.5.1 Verification of TH-cre;fl/fl mouse line. DNA was extracted from tail tissue of progeny from $\mathrm{TH}-\mathrm{Cre}+$; $\mathrm{FBXO} 7 \mathrm{fl} /+\mathrm{x}$ FBXO7 fl/fl breedings and subjected to PCR analysis with primers directed against Cre, FBXO7 wild type and the FBXO7/loxp2 cassette.

\subsubsection{TH-Cre;fl/fl mice show abnormal weight-regulation and hind limb clasping}

To characterize the $\mathrm{TH}-\mathrm{Cre}+$; FBXO7 $\mathrm{fl} / \mathrm{fl}(\mathrm{TH}-\mathrm{Cre} ; \mathrm{fl} / \mathrm{fl})$ mice, I subjected the animals together with age-matched $\mathrm{TH}-\mathrm{Cre}+; \mathrm{FBXO} 7$ wt ( $\mathrm{TH}-\mathrm{Cre}$ ) control groups to a row of behavioral analysis at 2, 6 and 12 months of age. At 2 months TH-Cre;fl/fl mice were phenotypically indistinguishable from $\mathrm{TH}$-Cre mice. When a new cohort of animals was tested at 6 months however, they showed a significant increase in weight (Figure 3.5.2). Since an increase in weight could interfere with motor tests, only ten animals from each group with comparable weights were included in statistical analysis for all tests at 6 months. Up until 6 months of age no animals died in any of the test cohorts, however from 
6 until 12 months $6 \mathrm{TH}-\mathrm{Cre}$;fl/fl mice were either found dead in their cage or sacrificed due to infections (Table 3.5.1).

Table 3.5.1 Reason for sacrifice of TH-Cre mice. Mice used for behavioral analysis were monitored over the course of 6 months and cause of death noted.

\begin{tabular}{llll}
\hline Animal & Genotype & Reason for sacrifice & Age of death \\
\hline 412 & TH-Cre;fl/fl & Found dead & 10 months \\
1870 & TH-Cre;fl/fl & Inflamed penis & 7 months \\
1872 & TH-Cre;fl/fl & Inflamed penis & 10 months \\
1873 & TH-Cre;fl/fl & Found dead & 11 months \\
2129 & TH-Cre;fl/fl & Found dead & 12 months \\
515 & TH-Cre;fl/fl & Inflamed anus & 8 months \\
521 & TH-Cre & Heart murmur & 9 months \\
\hline
\end{tabular}

This resulted in the 12 month old cohort being reduced to $9 \mathrm{TH}-\mathrm{Cre} ; \mathrm{fl} / \mathrm{fl}$ animals and 13 controls. When their bodyweight was measured again at 12 months, I found that the THCre;fl/fl mice were divided into two groups, one with lower and one with higher body weight (Figure 3.5.2). These groups however, performed comparable on all tests, so for statistical analysis the results were pooled.

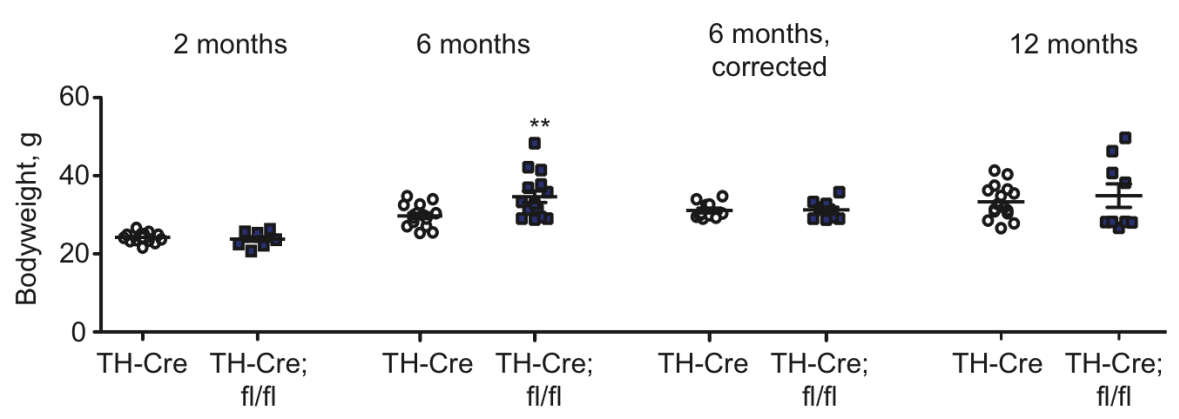

Figure 3.5.2 TH-Cre;fl/fl mice show increased weight at 6 months and are either over- or underweight at 12 months of age. TH-Cre and TH-Cre;fl/fl mice were weighed at 2, 6 and 12 months. At 6 months the heavier TH-Cre;fl/fl and the lighter TH-Cre mice were excluded from the statistical analysis. $\mathrm{N}(2$ months $)=15,9, \mathrm{~N}(6$ months $)=16,15, \mathrm{~N}(6$ months, corrected $)=11,10, \mathrm{~N}(12$ months) $=16,9$ for $\mathrm{TH}-\mathrm{Cre}$ and $\mathrm{TH}-\mathrm{Cre} ; \mathrm{fl} / \mathrm{fl}$ respectively. Data represent mean $\pm \mathrm{SEM}$ (t-test, $\left.{ }^{* *} \mathrm{p}<0.01\right)$.

Dopamine is also involved in controlling reward response upon feeding (Hajnal et al., 2004). I therefore wanted to see if the weight decrease seen in some animals was due to a lack of reward stimulation by food intake. I gave the mice the choice of regular water or sucrose-supplemented water, but found that the TH-Cre;fl/fl mice preferred the sucrose as much as the controls did (Figure 3.5.3). 


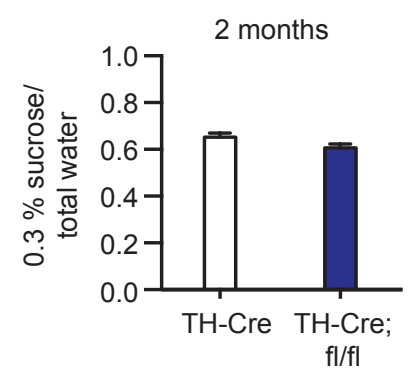

Figure 3.5.3 TH-Cre;fl/fl mice are not anhedonic at 2 months of age. $\mathrm{TH}-\mathrm{Cre}(\mathrm{N}=7)$ and $\mathrm{TH}-\mathrm{Cre} ; \mathrm{fl} / \mathrm{fl}(\mathrm{N}=9)$ mice were given the choice of water with or without $2 \%$ sucrose added. Bottles were weighed for four consecutive days after three days acclimatization. Ratio of sucrosesupplemented water to total water consumption is shown. Data represent mean \pm SEM (t-test).

At two months of age, no sign of hind limb clasping was present (Figure 3.5.4), but at six months a few TH-Cre;fl/fl mice starting showing a clasping phenotype. In the 12 months old mice, I observed a significant increase in their hind limb clasping indicating neurological damage.

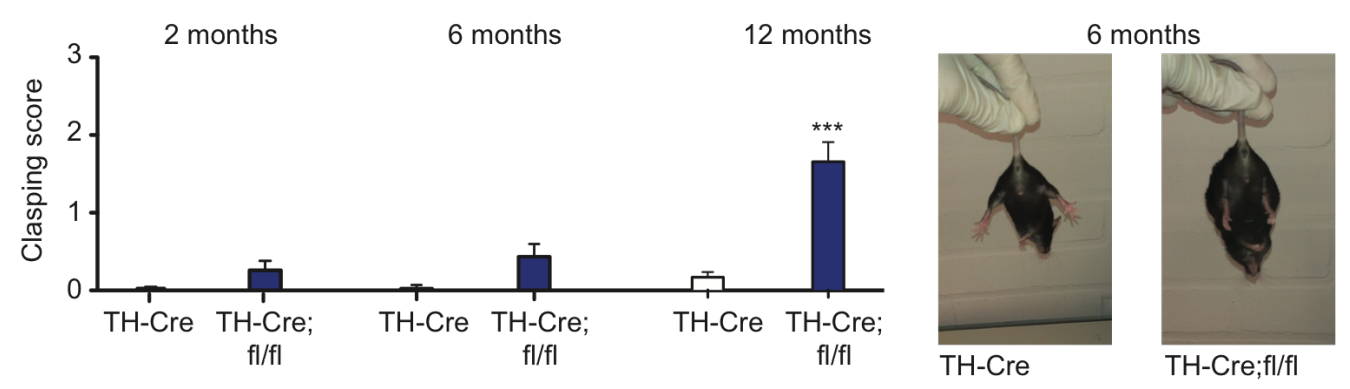

Figure 3.5.4 TH-Cre;fl/fl mice show hind limb clasping at 12 months of age. TH-Cre and $\mathrm{TH}-$ Cre;fl/fl mice were subjected to the tail suspension test for 10 seconds with three repetitions 30 seconds apart and scored for their hind limb clasping from 0 (normal) to 3 (severe). A representative picture of hind limb clasping at 6 months is shown. $\mathrm{N}(2$ months $)=15,9, \mathrm{~N}(6$ months, corrected $)=11,10, \mathrm{~N}(12$ months $)=16,9$ for $\mathrm{TH}-\mathrm{Cre}$ and $\mathrm{TH}-\mathrm{Cre} ; \mathrm{fl} / \mathrm{fl}$ respectively. Data represent mean \pm SEM (Mann-Whitney non-parametric t-test, $\left.{ }^{* * *} p<0.001\right)$.

With these results, I conclude that food intake and/or metabolism is disturbed in the adult $\mathrm{TH}-\mathrm{Cre}$;fl/fl mice. In addition they show hind limb clasping as a sign of progressive neurological dysfunction.

\subsubsection{TH-Cre;fl/fl mice show normal olfaction and anxiety phenotype}

Increase or decrease in anxiety can affect several motor tests, so I analyzed the time spent in different zones in an open field and found no difference between the genotypes at either age (Figure 3.5.5a). This result was also corroborated by the elevated plus maze test for the mice at 6 months, where the TH-Cre;fl/fl mice spent an equal amount of time in the closed arms versus the open arms of the maze as compared to control mice (Figure 3.5.5b). Also the distance traveled in the elevated plus maze was comparable between genotypes (Figure 3.5.5c). This indicates that TH-Cre;fl/fl mice show normal anxiety levels. 

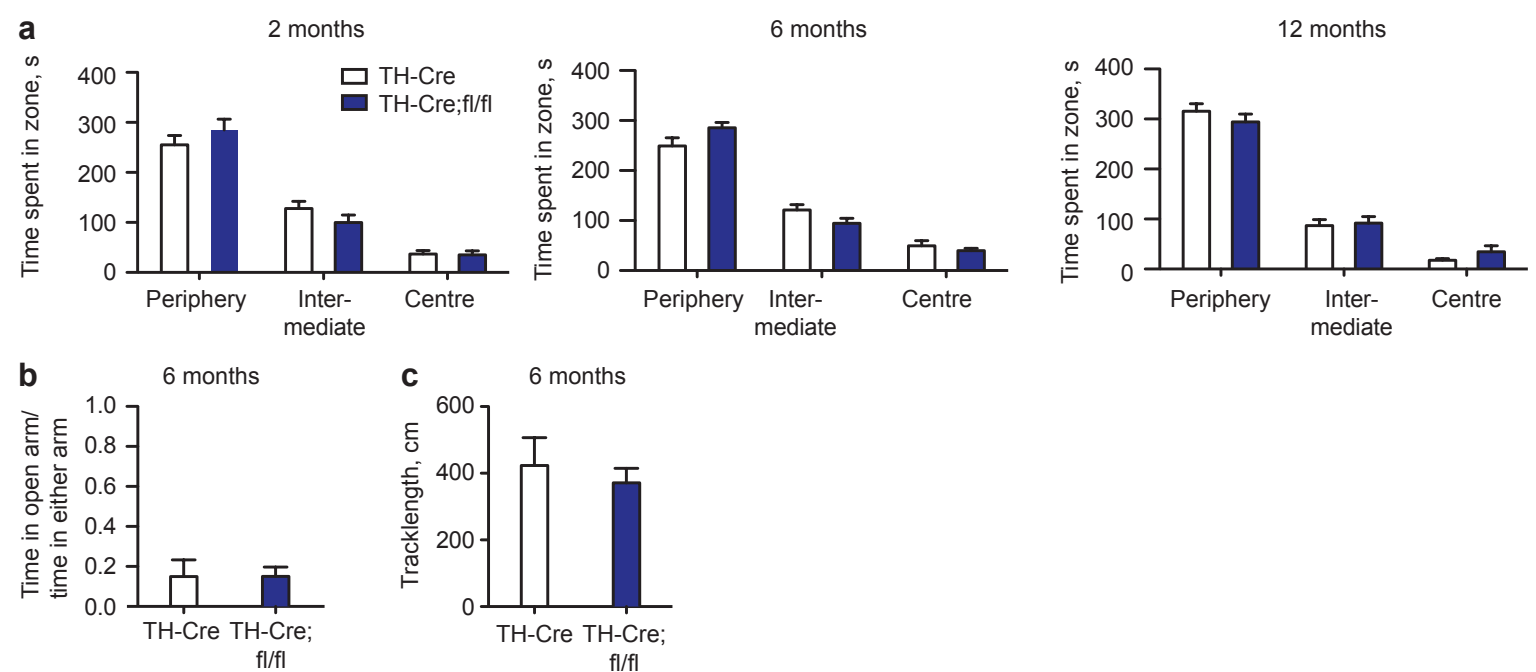

Figure 3.5.5 TH-Cre;fl/fl mice show no anxiety phenotype. Mice were placed in an open field of $60 \mathrm{~cm}$ diameter for 7 minutes and their ambulation pattern recorded with the viewer software at 2 , 6 and 12 months of age (a). $\mathrm{N}(2$ months $)=15,9, \mathrm{~N}(6$ months, corrected $)=11,10, \mathrm{~N}(12$ months $)=16,9$ for TH-Cre and TH-Cre;fl/fl respectively. Data represent mean \pm SEM (t-test). Mice were placed in an elevated plus maze with two open and two closed arms at 6 months of age (b,c). Time spent in open arms versus time spent in all arms (b) and total travel length (c) was measured. N(6 months, corrected $)=11,10$ for $\mathrm{TH}$-Cre and TH-Cre;fl/fl, respectively. Data represent mean \pm SEM (t-test).

Loss of olfaction is one of the earliest symptoms seen in Parkinson's disease, hence I assessed the TH-Cre;fl/fl mouse model for smell sensitivity. I performed a buried treat retrieval test, but found no difference between homozygous and control mice at neither of the ages tested (Figure 3.5.6).
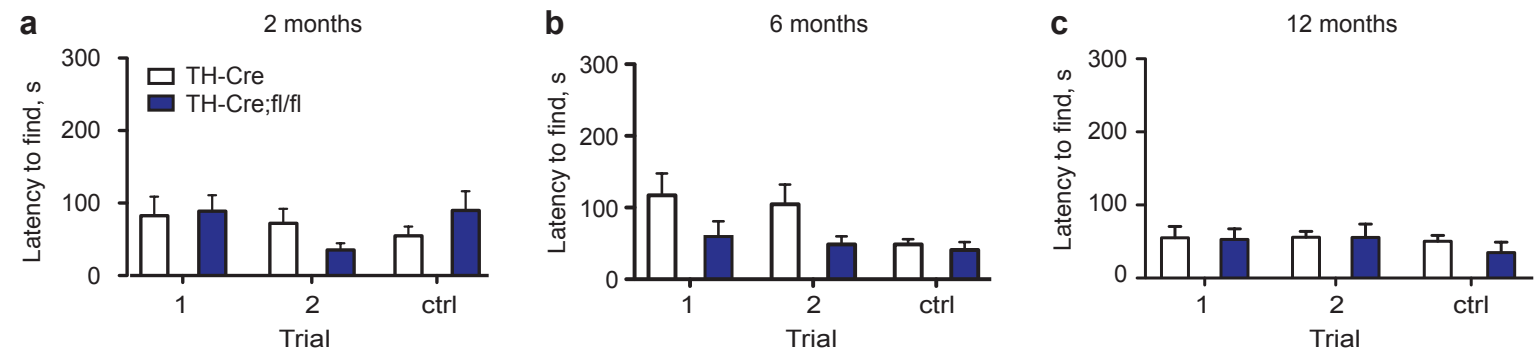

Figure 3.5.6 TH-Cre;fl/fl mice show no loss of olfaction. Mice of 2 (a), 6 (b) and 12 months (c) of age were placed in a cage where a chocolate chip was buried underneath soft bedding. Latency to find treat was measured in two trials after two days of training. The third trial was a control where chocolate chip was placed on top of bedding. $\mathrm{N}(2$ months $)=15,9, \mathrm{~N}(6$ months, corrected $)=11,10$, $\mathrm{N}(12$ months $)=16,9$ for TH-Cre and TH-Cre;fl/fl, respectively. Data represent mean \pm SEM (t-test). 


\subsubsection{TH-Cre;fl/fl mice display a progressive loss of motor function}

To assess overall ambulation, mice were placed in the open field for 7 minutes (Figure 3.5.7). At 2 months and 6 months, they all showed similar activity levels as they traveled comparable distances in the open field test. However, at 12 months the ambulation was severely reduced as the $\mathrm{TH}-\mathrm{Cre} ; \mathrm{fl} / \mathrm{fl}$ mice traveled only half the distance compared to controls.

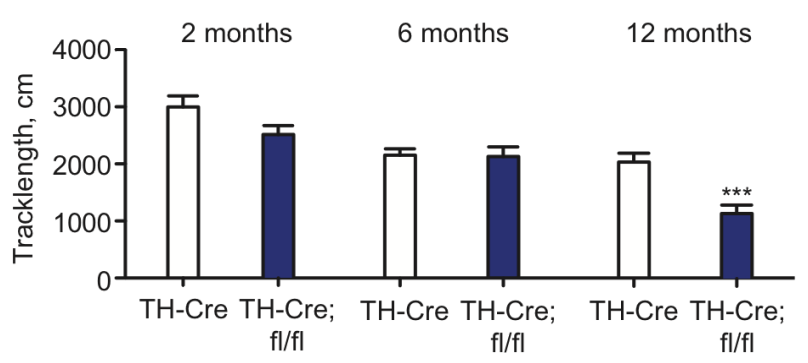

Figure 3.5.7 TH-Cre;fl/fl mice move less at 12 months of age. TH-Cre and TH-Cre;fl/fl mice of $2(\mathrm{~N}=15,9), 6(\mathrm{~N}=11,10)$ and 12 $(\mathrm{N}=16,9)$ months of age were placed in an open field of $60 \mathrm{~cm}$ diameter for 7 minutes and recorded with the Viewer software. Distance traveled was measured. Data represent mean \pm SEM (t-test, $\left.{ }^{* * *} p<0.001\right)$.

To further evaluate the motor function of TH-Cre;fl/fl mice, I subjected them to a coordination test. Here the mice had to balance across a beam and I measured their time to cross as well as their coordination while doing so. At 2 and 6 months of age the THCre;fl/fl mice performed as well as their controls (Figure 3.5.8a-d), but at 12 months it became clear that they spent significantly more time crossing the beam than the TH-Cre control mice (Figure 3.5.8e). The coordination, however, remained normal also for the 12 months old mice (Figure 3.5.8f).
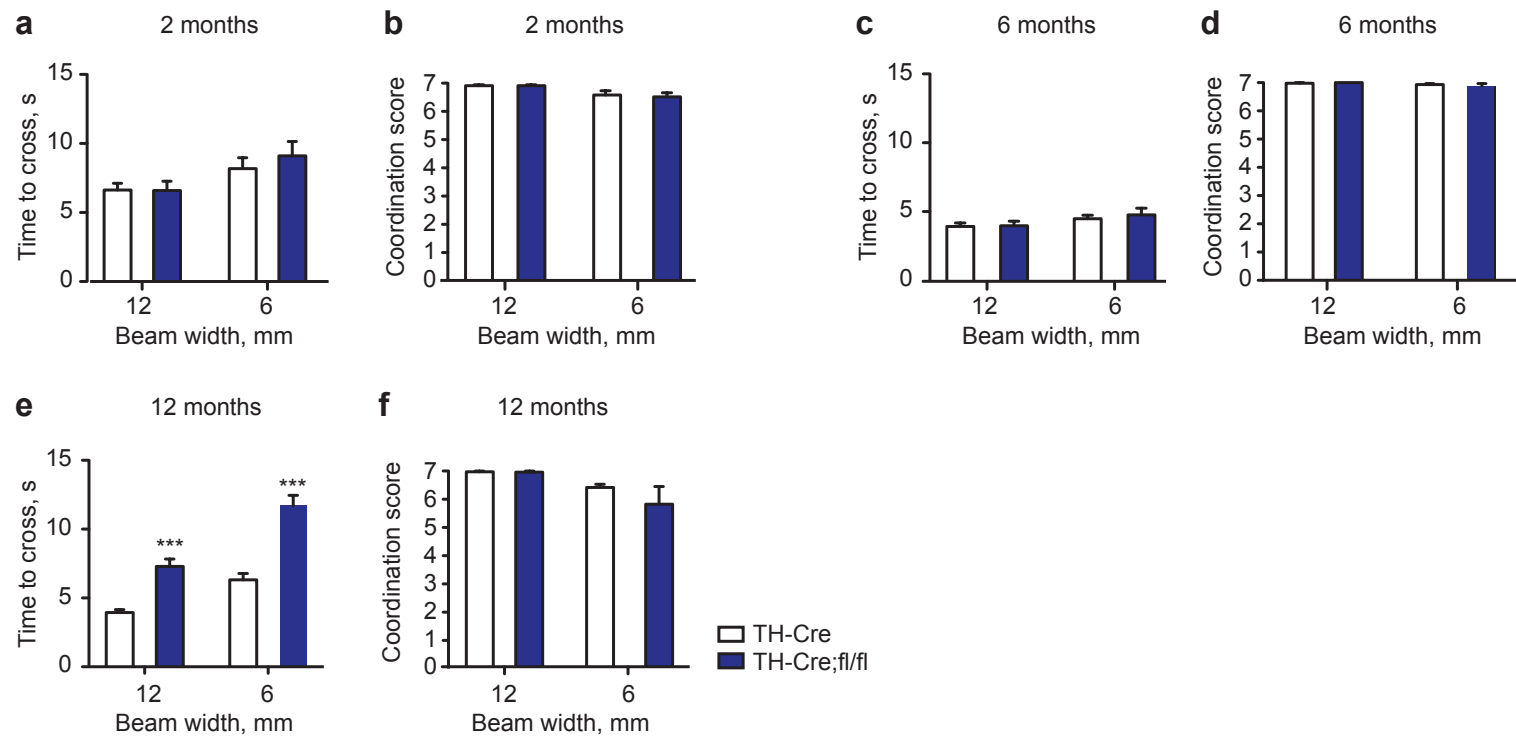

Figure 3.5.8 TH-Cre;fl/fl mice show progressively slower movements. Mice of 2 months (a,b), 6 months (c,d) or 12 months (e,f) of age were made to cross a beam of $80 \mathrm{~cm}$ length and either 6 or $12 \mathrm{~mm}$ width. Time to cross the beam $(\mathrm{a}, \mathrm{c}, \mathrm{e})$ as well as coordination score $(\mathrm{b}, \mathrm{d}, \mathrm{f}), 7=$ normal, $0=$ not able to cross, was measured. $\mathrm{N}(2$ months $)=15,9, \mathrm{~N}(6$ months, corrected $)=11,10, \mathrm{~N}(12$ 
months) $=16,9$ for $\mathrm{TH}-\mathrm{Cre}$ and $\mathrm{TH}-\mathrm{Cre} ; \mathrm{fl} / \mathrm{fl}$ respectively. Data represent mean $\pm \mathrm{SEM}$ (MannWhitney non-parametric t-test for score, t-test for time, $\left.{ }^{* * *} p<0.001\right)$.

A more challenging way of measuring motor performance is the rotarod test. Here, the mice are placed on an accelerating barrel and latency to fall measured. In this test, I measured both their initial performance as well as their motor learning. At 2 months of age $\mathrm{TH}-\mathrm{Cre} ; \mathrm{fl} / \mathrm{fl}$ mice showed the same motor performance as their controls, but at 6 months there was already a significant difference in their ability to stay on the rod (Figure 3.5.9 a,b). At twelve months this difference was further increased and even after three test days the $\mathrm{TH}-\mathrm{Cre}$;fl/fl could not reach their former performance level, while the wild types performed as well at 12 months as they did at 6 months (Figure $3.5 .9 \mathrm{c}$ ).
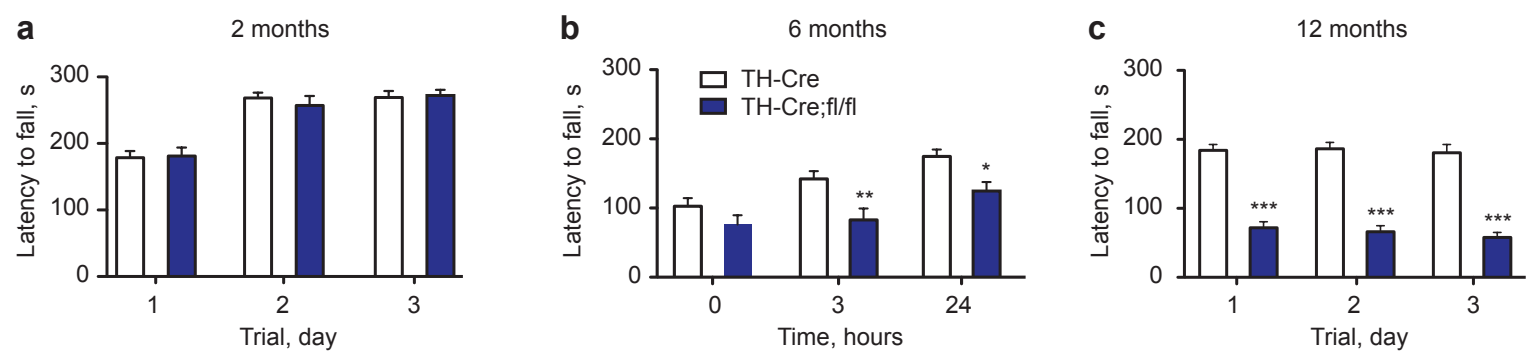

Figure 3.5.9 TH-Cre;fl/fl mice show progressive loss of motor function. Mice of 2 months (a), 6 months (b) and 12 months (c) of age were tested on an accelerating rotarod going from 4-40 rpm in 5 minutes. At 2 and 12 months mice were tested 3 times a day for 3 days, while at 6 months they were tested three times with 3 and 24 hours in between trials. $\mathrm{N}(2$ months $)=15,9, \mathrm{~N}(6$ months, corrected $)=11,10, \mathrm{~N}(12$ months $)=16,9$ for $\mathrm{TH}-\mathrm{Cre}$ and $\mathrm{TH}-\mathrm{Cre} ; \mathrm{fl} / \mathrm{fl}$ respectively. Data represent mean \pm SEM (t-test, $\left.{ }^{*} p<0.05,{ }^{* *} p<0.01,{ }^{* * *} p<0.001\right)$.

Since both the rotarod and the balance beam are general tests of motor function, I wanted to specifically assess the possible dopaminergic dysfunction through the pole test (Ogawa et al., 1985). Here, mice have to turn and climb down a pole challenging the fine movement adjustments regulated by the basal ganglia. In this test, I found no difference at 6 months of age, but a significant decline at 12 months of age, suggesting that we indeed have a progressive basal ganglia disturbance (Figure 3.5.10).
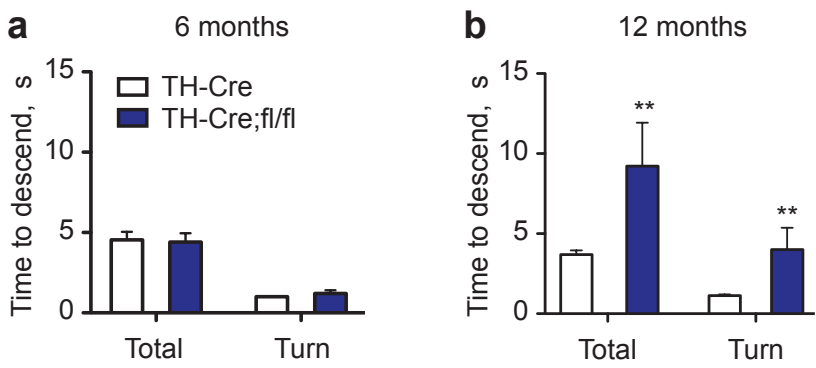

Figure 3.5.10 TH-Cre;fl/fl mice show progressive loss of fine motor control. Mice of 6 (a) and 12 months (b) of age were placed on a $50 \mathrm{~cm}$ tall pole head up and time to turn around and descend pole was measured. $\mathrm{N}(6$ months, corrected $)$ $=11,10, \mathrm{~N}(12$ months $)=16,9$ for $\mathrm{TH}-\mathrm{Cre}$ and TH-Cre;fl/fl respectively. Data represent mean \pm SEM (t-test, $\left.{ }^{* *} p<0.01\right)$. 
Recently several systems for testing of gait parametrics has been developed, amongst them the DigiGait system (Kale et al., 2004). This system allows for fine measurements that can identify specific gait parameters associated with Parkinson's disease (Amende et al., 2005). We established collaboration with Camille Lancelin in the Marquardt lab to take advantage of this technique. She let 8 months old animals run on the DigiGait at standard speed and with a more challenging elevated protocol. She then analyzed the results and found differences between several of the parameters (Table 3.5.2). Notably the fore limbs of the TH-Cre;fl/fl mice showed a significant decrease in stride, brake and stance, while the hind limbs showed a decrease in stride, propel and stance as well as an increased paw drag.

Table 3.5.2 TH-Cre;fl/fl mice show alteration in gait parameters at 8 months of age. THCre;fl/fl $(\mathrm{N}=7)$ and age-matched TH-Cre $(\mathrm{N}=7)$ controls were subjected to Digigait analysis at 8 months of age. Data is shown as mean and p-value (t-test, ${ }^{*} \mathrm{p}<0.05,{ }^{* *} \mathrm{p}<0.01$ ). Experiment conducted by Dr. Camille Lancelin.

\begin{tabular}{|c|c|c|c|c|}
\hline \multirow[b]{2}{*}{ Parameter } & \multicolumn{2}{|c|}{ Fore limbs } & \\
\hline & Unit & $\mathrm{p}$-value & TH-cre & TH-cre;fl/fi \\
\hline MIDLINE.DISTANCE & $(\mathrm{cm})$ & $0.003 * *$ & -2.914 & -3.348 \\
\hline BRAKE & (s) & $0.007 * *$ & 0.048 & 0.038 \\
\hline STANCE & (s) & $0.012 *$ & 0.142 & 0.126 \\
\hline OVERLAP.DISTANCE & $(\mathrm{cm})$ & 0.028 * & 1.419 & 1.873 \\
\hline X.BRAKE.STRIDE & $(\%)$ & 0.044 * & 19.329 & 16.771 \\
\hline STRIDE & (s) & 0.049 * & 0.252 & 0.229 \\
\hline STRIDE.LENGTH & $(\mathrm{cm})$ & 0.057 & 6.300 & 5.736 \\
\hline STRIDE.FREQUENCY & (steps/s) & 0.063 & 4.079 & 4.457 \\
\hline X.PROPEL.STANCE & $(\%)$ & 0.073 & 65.743 & 69.636 \\
\hline X.BRAKE.STANCE & $(\%)$ & 0.073 & 34.257 & 30.364 \\
\hline PAW.AREA.VAR & $\left(\mathrm{cm}^{\wedge} 2\right)$ & 0.086 & 0.016 & 0.020 \\
\hline ABS.AXIS.DISTANCE & $(\mathrm{cm})$ & 0.138 & 0.911 & 0.866 \\
\hline NORM.STRIDE.LENGTH & (real\#) & 0.167 & 0.553 & 0.488 \\
\hline MIN.DA.DT & $\left(\mathrm{cm}^{\wedge} 2 / \mathrm{s}\right)$ & 0.168 & -4.522 & -4.989 \\
\hline ABS.PAW.ANGLE & (deg) & 0.235 & 10.493 & 7.557 \\
\hline NORM.STANCE.WIDTH & (real\#) & 0.282 & 0.438 & 0.416 \\
\hline STANCE.SWING & (real\#) & 0.293 & 1.307 & 1.221 \\
\hline PROPEL & (s) & 0.319 & 0.093 & 0.088 \\
\hline SWING & (s) & 0.333 & 0.110 & 0.103 \\
\hline PAW.AREA & $\left(\mathrm{cm}^{\wedge} 2\right)$ & 0.363 & 0.234 & 0.247 \\
\hline STANCE.WIDTH.CV & $(\mathrm{CV} \%)$ & 0.370 & 16.394 & 13.229 \\
\hline X.SWING.STRIDE & $(\%)$ & 0.386 & 43.571 & 44.929 \\
\hline X.STANCE.STRIDE & $(\%)$ & 0.390 & 56.429 & 55.079 \\
\hline FORELIMB.WEIGHT.SUP & & 0.401 & 0.699 & 0.679 \\
\hline STANCE.WIDTH.VAR & $(\mathrm{cm})$ & 0.411 & 0.289 & 0.236 \\
\hline STRIDE.LENGTH.CV & (CV\%) & 0.415 & 12.004 & 13.201 \\
\hline STEP.ANGLE.CV & (CV\%) & 0.429 & 23.609 & 19.264 \\
\hline X.PROPEL.STRIDE & $(\%)$ & 0.457 & 37.086 & 38.300 \\
\hline MAX.DA.DT & $\left(\mathrm{cm}^{\wedge} 2 / \mathrm{s}\right)$ & 0.538 & 15.366 & 16.048 \\
\hline STEP.ANGLE & (deg) & 0.561 & 57.500 & 60.800 \\
\hline PAW.ANGLE.VAR & (deg) & 0.643 & 8.721 & 9.379 \\
\hline STEP.ANGLE.VAR & (deg) & 0.690 & 12.330 & 11.543 \\
\hline SWING.DURATION.CV & (CV\%) & 0.729 & 14.884 & 14.144 \\
\hline ATAXIA.COEFF & (real\#) & 0.772 & 0.404 & 0.418 \\
\hline PAW.ANGLE & (deg) & 0.805 & 0.264 & -0.300 \\
\hline STANCE.WIDTH & $(\mathrm{cm})$ & 0.805 & 1.771 & 1.800 \\
\hline STANCE.FACTOR & (real\#) & 0.808 & 1.003 & 1.009 \\
\hline GAIT.SYMMETRY & (real\#) & 0.853 & 1.004 & 1.003 \\
\hline STRIDE.LENGTH.VAR & $(\mathrm{cm})$ & 0.940 & 0.748 & 0.754 \\
\hline PPP & $(\mathrm{cm})$ & 0.975 & 0.506 & 0.508 \\
\hline
\end{tabular}

\begin{tabular}{|c|c|c|c|c|}
\hline \multirow[b]{2}{*}{ Parameter } & \multicolumn{2}{|c|}{ Hind limbs } & \multicolumn{2}{|c|}{ Mean } \\
\hline & Unit & $\mathrm{p}$-value & TH-cre & TH-cre;fl/f \\
\hline PAW.DRAG & (real\#) & $0.005 * *$ & -5.160 & -6.605 \\
\hline OVERLAP.DISTANCE & $(\mathrm{cm})$ & 0.028 * & 1.419 & 1.873 \\
\hline PAW.WIDTH & $(\mathrm{cm})$ & $0.028 *$ & 0.780 & 0.711 \\
\hline PAW.AREA & $\left(\mathrm{cm}^{\wedge} 2\right)$ & $0.030 *$ & 0.584 & 0.531 \\
\hline MAX.DA.DT & $\left(\mathrm{cm}^{\wedge} 2 / \mathrm{s}\right)$ & 0.036 * & 48.283 & 42.121 \\
\hline STANCE & (s) & 0.042 * & 0.156 & 0.145 \\
\hline PROPEL & (s) & 0.047 * & 0.121 & 0.103 \\
\hline STRIDE & (s) & 0.047 * & 0.252 & 0.229 \\
\hline STRIDE.LENGTH & $(\mathrm{cm})$ & 0.053 & 6.307 & 5.743 \\
\hline NORM.STANCE.WIDTH & (real\#) & 0.061 & 0.694 & 0.646 \\
\hline STEP.ANGLE.CV & (CV\%) & 0.070 & 22.617 & 33.913 \\
\hline STRIDE.FREQUENCY & (steps/s) & 0.083 & 4.086 & 4.443 \\
\hline STEP.ANGLE & (deg) & 0.08 & 56.657 & 47.100 \\
\hline SWIN & (s) & 0.1 & 0.097 & 0.085 \\
\hline E.WIDTH.CV & (CV\%) & 0.1 & .950 & 7.143 \\
\hline PAW & $(\mathrm{cm})$ & 0.1 & 1.458 & 1.391 \\
\hline LE.VAR & (de & 0.1 & 12.530 & 14.960 \\
\hline NOP & (real\#) & 0.1 & 554 & 0.489 \\
\hline X.BRAKE.STRIDE & $(\%)$ & 0.1 & 13.650 & 18.014 \\
\hline STA & (cn & & 167 & 0.200 \\
\hline PAV & $(\mathrm{cm})$ & 0.1 & 0.051 & 0.044 \\
\hline X.B & $(\%)$ & 0.1 & 22.129 & 28.836 \\
\hline X.PROPEL.STANCE & $(\%)$ & 0.191 & 77.871 & 71.164 \\
\hline BRA & & 0.272 & 0.034 & 0.042 \\
\hline PAW.LB & $(\mathrm{cm})$ & 0.290 & 0.124 & 0.111 \\
\hline ABS.AXIS.DIS & $(\mathrm{cm})$ & 0.323 & 1.461 & 1.390 \\
\hline X.SHARED.STANCE & $(\%)$ & 0.336 & 33.736 & 37.736 \\
\hline STANCE.SWING & (real\#) & 0.344 & 1.636 & 1.743 \\
\hline X.PROPEL.STRIDE & $(\%)$ & 0.381 & 48.193 & 45.114 \\
\hline X.STANCE.STR & $(\%)$ & 0.415 & 61.864 & 63.129 \\
\hline X.SWING.STRIL & $(\%)$ & 0.415 & 38.136 & 36.871 \\
\hline STRID & (CV\%) & 0.484 & 12.006 & 13.413 \\
\hline SHARED.STANCE & (s) & 0.603 & 0.052 & 0.055 \\
\hline SFI & $(\mathrm{cm})$ & 0.669 & -8.795 & -8.650 \\
\hline TFI & $(\mathrm{cm})$ & 0.674 & -8.799 & -8.661 \\
\hline MIDLINE.DISTANC & $(\mathrm{cm})$ & 0.6 & 1.529 & 1.656 \\
\hline .DURATION.CV & (CV\%) & 0.695 & 11.966 & 12.931 \\
\hline PAW.ANGLE.VAR & (deg) & 0.698 & 4.164 & 4.343 \\
\hline TAU.PROPULSION & (real\#) & 0.707 & 0.161 & 0.148 \\
\hline E.FACTOR & (real\#) & 0.753 & 1.033 & 1.024 \\
\hline MIN.DA.DT & $\left(\mathrm{cm}^{\wedge} 2 / \mathrm{s}\right)$ & 0.756 & -8.642 & -8.517 \\
\hline PFI & $(\mathrm{cm})$ & 0.797 & -11.781 & -12.079 \\
\hline ABS.PAW.ANGLE & (deg) & 0.798 & 15.471 & 15.121 \\
\hline GAIT.SYMMETRY & (real\#) & 0.853 & 1.004 & 1.003 \\
\hline PAW.ANGLE & (deg) & 0.928 & 0.929 & 0.807 \\
\hline ATAXIA.COEFF & (real\#) & 0.940 & 0.411 & 0.416 \\
\hline PPP & $(\mathrm{cm})$ & 0.975 & 0.506 & 0.508 \\
\hline STRIDE.LENGTH.VAR & $(\mathrm{cm})$ & 0.993 & 0.759 & 0.760 \\
\hline PAW.AREA.VAR & $\left(\mathrm{cm}^{\wedge} 2\right)$ & 1.000 & 0.036 & 0.036 \\
\hline STANCE.WIDTH & $(\mathrm{cm})$ & 1.000 & 2.800 & 2.800 \\
\hline
\end{tabular}


Taken together, this battery of tests reveal that the TH-Cre;fl/fl mice show normal motor function during development, but start to show decline around 6 months of age. The finer gait parameters show a slower, stiffer gait that results in difficulties with more challenging motor tasks. At 12 months of age the symptoms have progressed so far that there is a general decrease in ambulation reminiscent of the bradykinesia and rigidity found in PARK15 patients.

\subsubsection{TH-Cre;fl/fl mice have reduced amount of dopamine in the striatum}

Parkinson's disease is caused by a degeneration of the dopaminergic neurons in the substantia nigra with a resulting loss of dopaminergic input into the striatum. To determine if the progressive motor symptoms seen in TH-Cre;fl/fl mice stem from loss of dopaminergic neurons, I conducted stereological counting of TH positive neurons in the substantia nigra of 2 and 12 months old mice. Both total number of TH positive neurons in the substantia nigra and the ratio of $\mathrm{TH}$ positive neurons to Nissl positive cells were quantified, but no difference was found between the two groups neither at 2 months nor at 12 months (Figure 3.5.11).
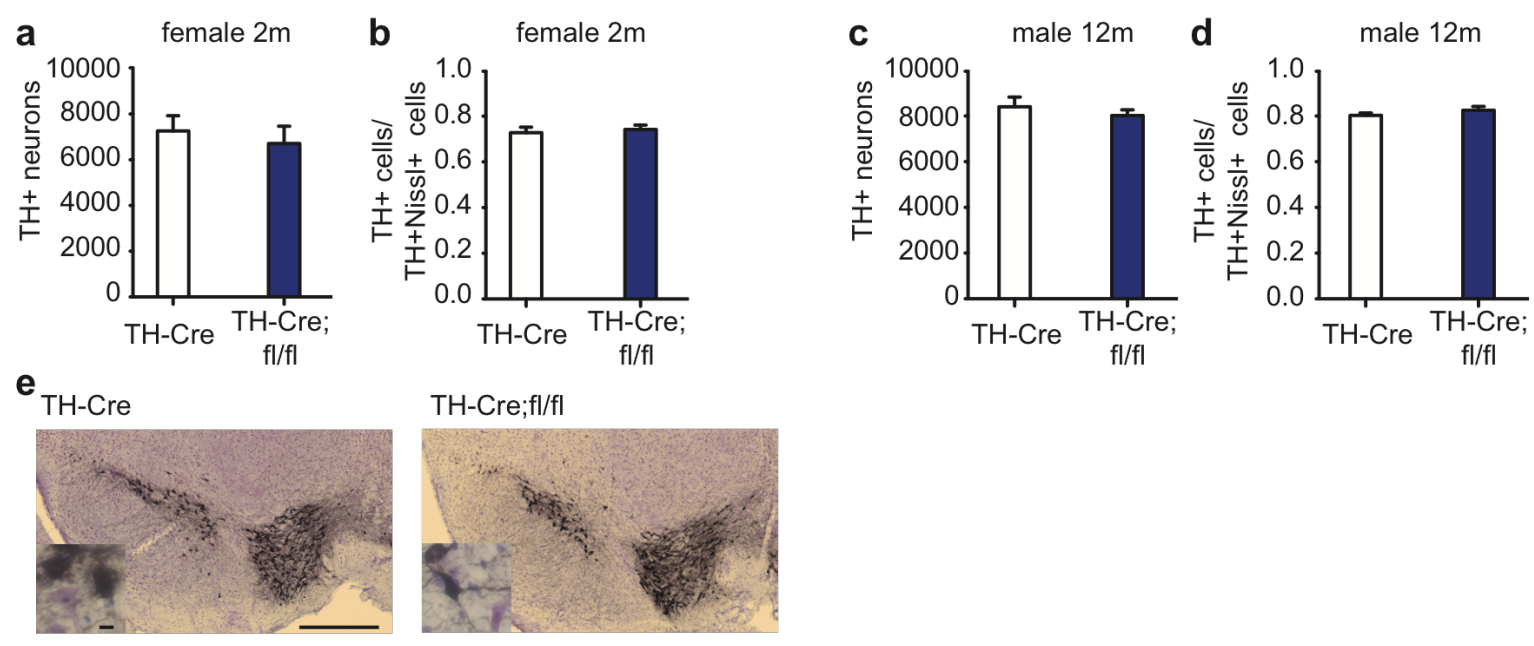

Figure 3.5.11 TH-Cre;fl/fl mice show no loss of neurons in substantia nigra. TH-Cre;fl/fl $(\mathrm{N}=3)$ and age-matched TH-Cre $(\mathrm{N}=3)$ controls of $2(\mathrm{a}, \mathrm{b})$ and $12(\mathrm{c}, \mathrm{d})$ months of age were trans-cardially perfused and brains fixed before coronal cryo-sectioning of 50 um thick sections. Every fifth section was then subjected to immunohistochemistry with an anti-TH antibody followed by Nissl staining with thioacetate. Counting of $\mathrm{TH}$ - and Nissl-positive neurons were performed with the Stereoinvestigator software and total number of TH positive cells $(a, c)$ as well as ratio of TH to Nissl positive cells $(b, d)$ counted. Representative pictures of midbrain of 12 months old animals (e). Scale bars equal $500 \mu \mathrm{m}$, inset $20 \mu \mathrm{m}$. Data represent mean \pm SEM (t-test). 
Although cell bodies did not seem to be lost in the TH-Cre;fl/fl mice, axonal loss could still occur. I therefore stained striatal sections from 12 months old mice and controls with an antibody against $\mathrm{TH}$. I found no visual difference between knockout mice and controls (Figure 3.5.12).

TH-Cre

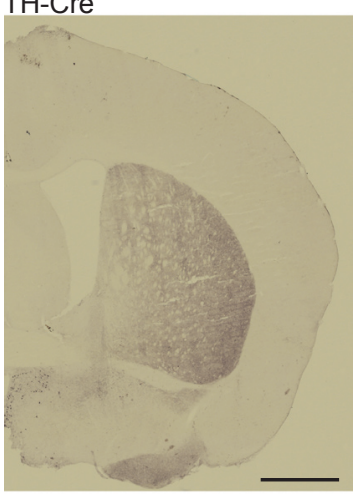

TH-Cre;fl/fl

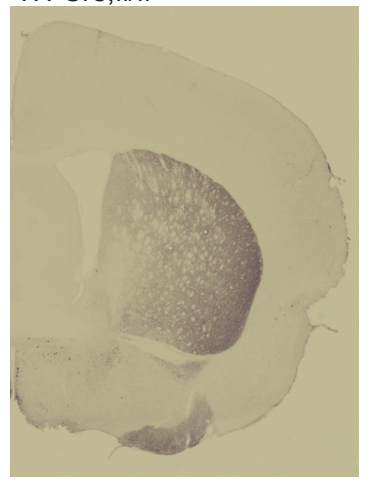

Figure 3.5.12 TH-Cre;fl/fl mice show little or no striatal terminal loss. TH-Cre; $\mathrm{fl} / \mathrm{fl}$ and agematched controls of $12(\mathrm{c}, \mathrm{d})$ months of age were trans-cardially perfused and brains fixed before coronal cryo-sectioning of $50 \mu \mathrm{m}$ thick sections. Every fifth section was then subjected to immunohistochemistry with an anti-TH antibody. Representative picture is shown.

To confirm this, I also lysed striatal tissue from 2 and 12 months old mice and performed immunoblotting analysis with antibodies against $\mathrm{TH}$ and the dopamine transporter (DAT) (Figure 3.5.13 and 14). Here, I found no difference in TH and DAT levels in 2 months old mice (Figure 3.5.13a,b).

Striatum TH 2 months
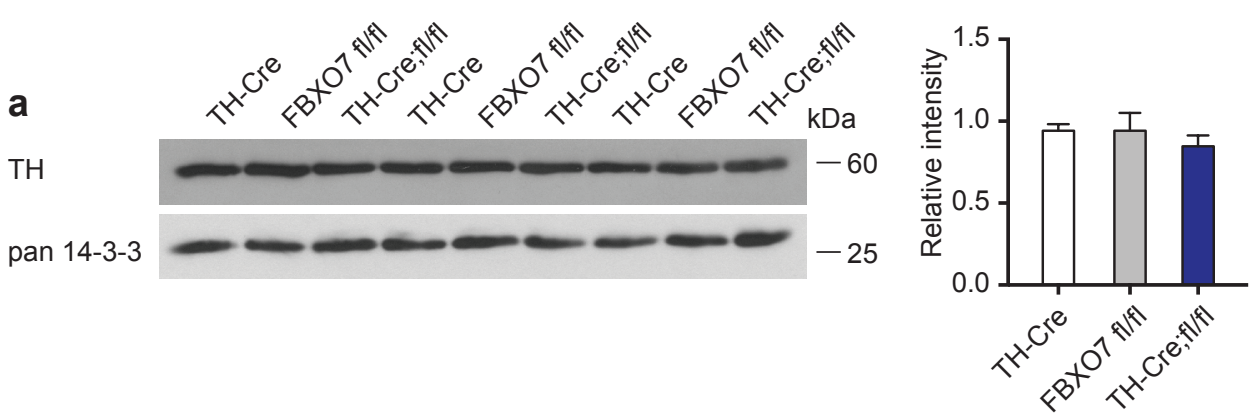

Striatum DAT 2 months
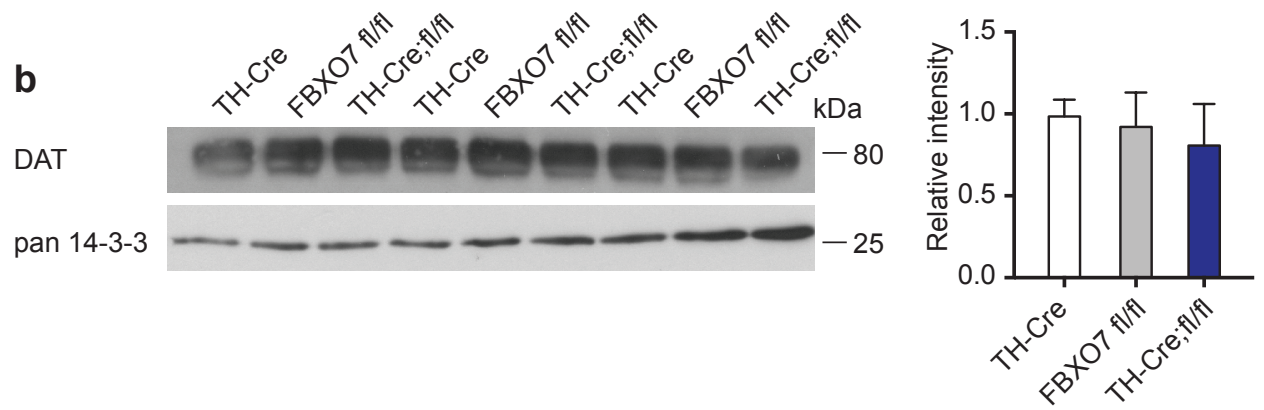

Figure 3.5.13 TH-Cre;fl/fl mice show normal expression of TH and DAT protein levels in the striatum at 2 months of age. Tissue from TH-Cre;fl/fl and age-matched controls of 2 months of age was lysed and subjected to SDS-PAGE analysis followed by immunoblotting with anti-TH or anti-DAT . Blots were quantified with the ImageJ software and intensity normalized to loading 
control (pan 14-3-3). Values are expressed relative to first TH-Cre sample. Data represent mean \pm SEM (t-test, $\left.{ }^{*} p<0.05\right)$.

In 12 months old mice however, DAT was significantly down-regulated (Figure 3.5.14b). This was not the result of a glutamatergic neuron imbalance as seen in the NEX-Cre animals as VGLUT1 levels were normal (Figure 3.5.14c).
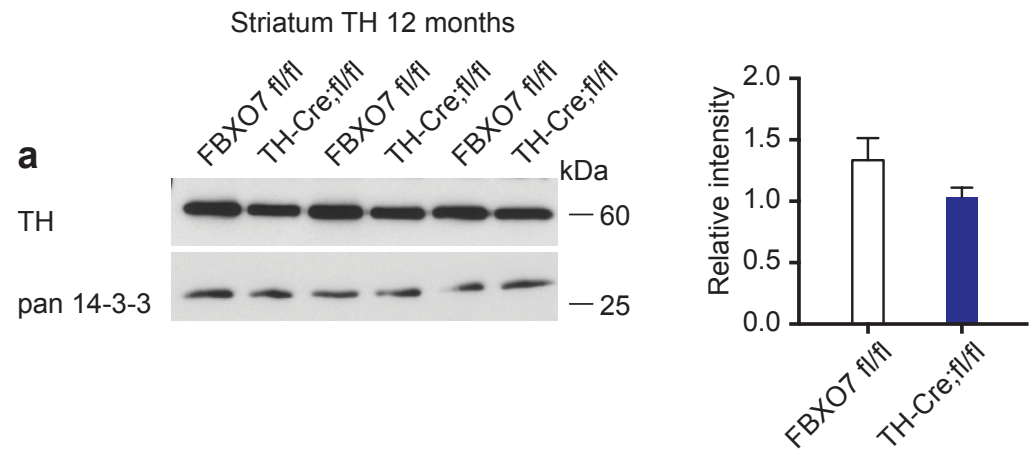

Striatum DAT 12 months
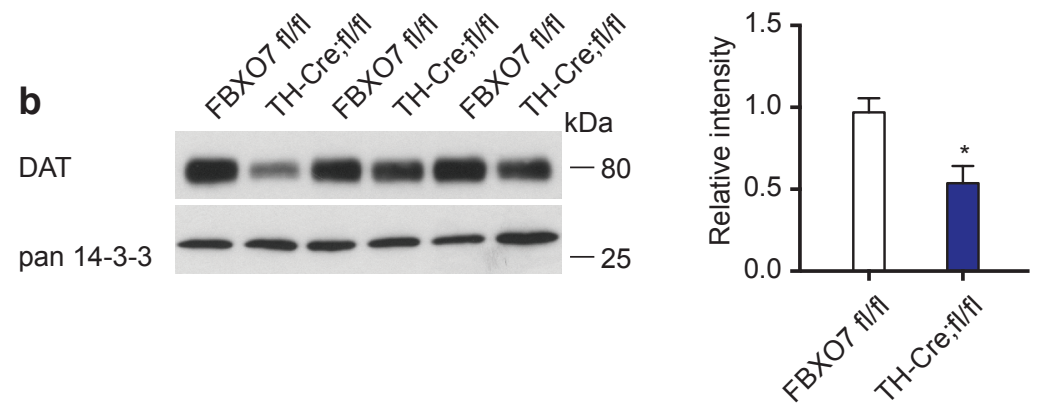

Striatum vGlut1 12 months
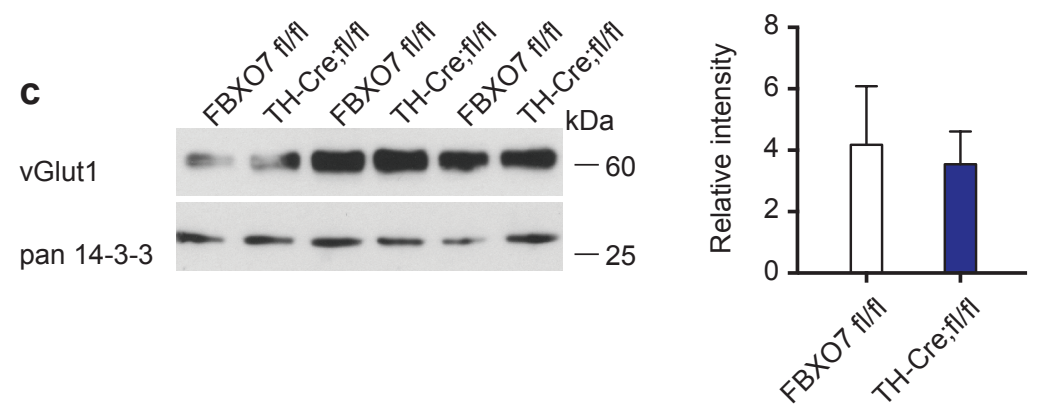

Figure 3.5.14 TH-Cre;fl/fl mice show downregulation of DAT protein levels in the striatum at 12 months of age. Tissue from TH-Cre,fl/fl and age-matched controls of 12 months of age was lysed and subjected to SDS-PAGE analysis followed by immunoblotting with anti-TH (a), anti-DAT (b) or anti-VGLUT1 (c) antibodies. Blots were quantified with the ImageJ software and intensity normalized to loading control (pan 14-3-3). Values are expressed relative to first TH-Cre sample. Data represent mean \pm SEM (t-test, $\left.{ }^{*} p<0.05\right)$. 
As the functional consequence of dopaminergic neuron loss is a loss of dopamine to the striatum, I prepared samples for HPLC analysis, which were then conducted by Dr. Lars Tatenhorst (Figure 3.5.15). We here found that in both 2 months and 12 months old animals the level of dopamine in the striatum was approximately half of the control level. This was accompanied by a slight decrease in the metabolite DOPAC level, but no change in HVA, leading to an increased metabolism.
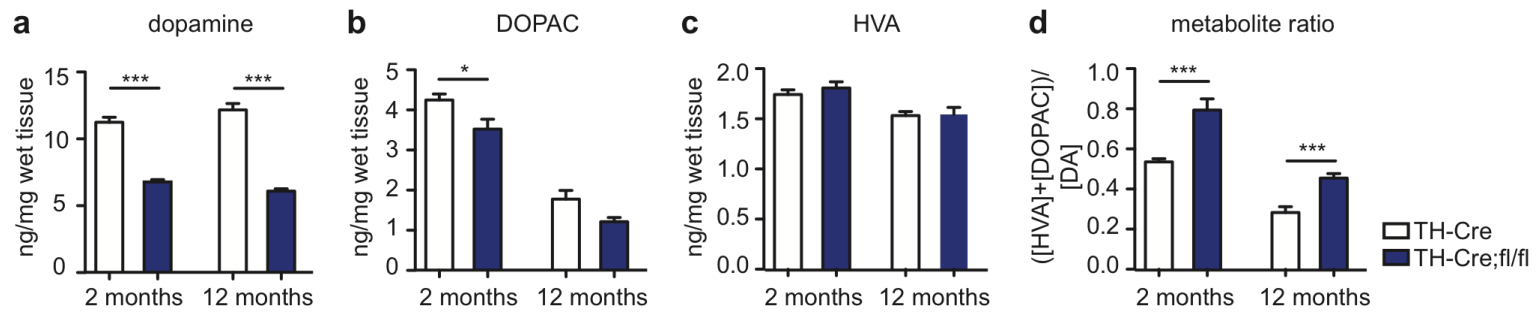

Figure 3.5.15 TH-Cre;fl/fl mice show downregulation of dopamine levels in the striatum. Striatal tissue from TH-Cre; $f l / f l(N=9,12)$ and age-matched TH-Cre $(\mathrm{N}=7,15)$ controls of 2 and 12 months of age respectively was subjected to HPLC analysis for dopamine (a), DOPAC (b) and HVA (c). Metabolism is shown as ratio of HVA and DOPAC to dopamine (d). Data represent mean \pm SEM (t-test, $\left.{ }^{*} p<0.05,{ }^{* * *} p<0.001\right)$.

Collectively, the TH-Cre;fl/fl mice display no change in dopaminergic cells in the substantia nigra, but a reduced level of striatal dopamine already at 2 months. The low dopamine level persists until 12 months of age at which point it is accompanied by a reduction in dopamine transporter levels in the striatum.

\subsubsection{TH-Cre;fl/fl mice show astrogliosis in the midbrain}

In both the conventional as well as the NEX-Cre; FBXO7 $\mathrm{fl} / \mathrm{fl}$ line, we found an increased response of glial cells. This accompanied by the fact that inflammation has been found in several PD mouse models (Kato et al., 2003; Kett et al., 2015) prompted us to look into the inflammatory response in the $\mathrm{TH}-\mathrm{Cre} ; \mathrm{fl} / \mathrm{fl}$ brain. I stained histological sections of midbrains from TH-Cre;fl/fl animals and controls at 12 months of age with antibodies against astrocytes (GFAP), microglia (Iba1) and activated microglia (Mac3) (Figure 3.5.16). I co-stained with anti-TH antibodies and used this to delineate the substantia nigra and ventral tegmental area. I then quantified the area of GFAP/Iba1/Mac3 positive staining inside the TH-marked area. I found an increase in GFAP levels in TH-Cre;fl/fl animals compared to control (Figure 3.5.16a,b), but no difference in lba1 or Mac3 staining (Figure 3.5.16c,d). 

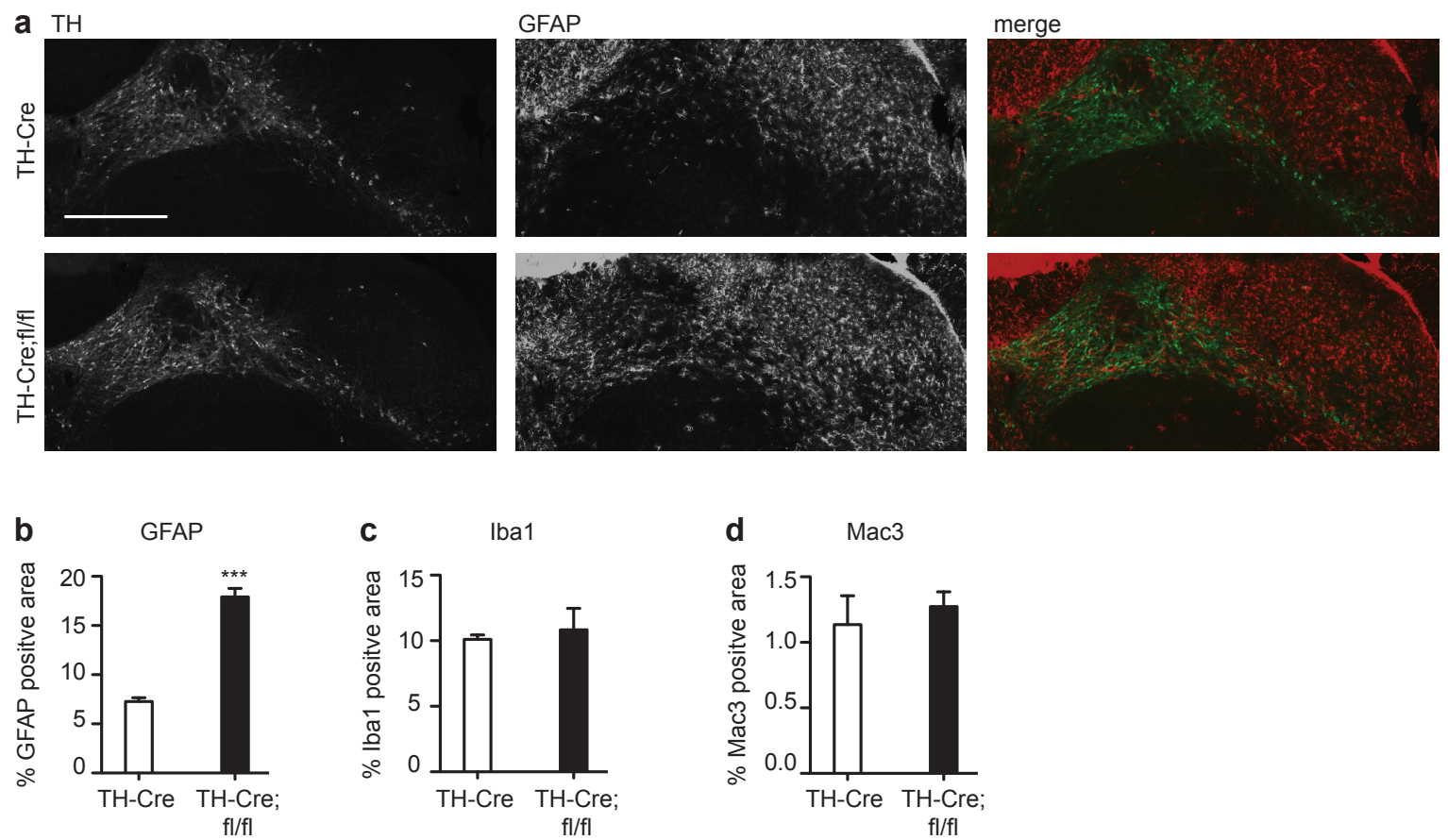

Figure 3.5.16 TH-Cre;fl/fl animals show increased GFAP positive area in the midbrain. THCre;fl/fl $(\mathrm{N}=3)$ and age-matched TH-Cre $(\mathrm{N}=3)$ controls of 12 months of age were trans-cardially perfused and brains were fixed prior to coronal cryo-sectioning of $50 \mu \mathrm{m}$ thick sections. Every fifth section was then subjected to immunohistochemistry with a TH, GFAP, Iba1 or Mac3 antibody. GFAP- (b), Iba1- (c) or Mac3-positive (d) area within TH-positive area was quantified. Representative pictures of midbrain of 12 months old mice stained for TH and GFAP is shown (a). Scale bars equal $500 \mu \mathrm{m}$, inset $20 \mu \mathrm{m}$. Data represent mean \pm SEM (t-test, $\left.{ }^{* * *} p<0.001\right)$.

These results show that for all our FBXO7 knockout mouse lines, we have an upregulation of astrocytes in the vicinity of the affected neurons. In the TH-Cre;fl/fl mice, however, this is not accompanied by reactive microglia at 12 months of age. 


\section{Discussion}

Parkinson's disease, a debilitating motor disease, is a tremendous burden on patients and their families, which also implies large socio-economic costs. In recent years, mechanisms underlying this disease have been heavily investigated and multiple hypotheses suggested. The ultimate goal of Parkinson's disease research would be to unify the current hypotheses and find common pathways for potential drug intervention. In the search of these pathways, genetic models have been of great help, but most models failed to faithfully recapitulate all the features of Parkinson's disease. A newly discovered PARK gene, FBXO7, causes Parkinsonian-Pyramidal Syndrome, but the disease mechanism remains unknown.

In this study, I have characterized the conventional FBXO7 knockout mouse as a novel mouse model for Parkinsonian-Pyramidal Syndrome. I have shown that the mice recapitulated many of the features associated with early onset Parkinson's disease such as motor impairment and premature death. In addition, I have investigated the impact of loss of FBXO7 on neuronal function by characterizing two newly generated conditional FBXO7 knockout mouse lines. I have shown that loss of FBXO7 in the mouse forebrain triggered loss of motor control reminiscent of the pyramidal symptoms seen in PARK15 patients. In contrast, I have shown that a loss of FBXO7 in catecholaminergic neurons caused progressive loss of motor function with gait defects, slowness of movements and reduced ambulation closely modeling Parkinsonian symptoms.

At the cellular level, I have demonstrated that loss of FBXO7 in catecholaminergic neurons led to reduced dopamine levels in the striatum accompanied by reactive astrogliosis of the substantia nigra, but without corresponding cell loss. Conversely, I have shown that knockout of FBXO7 in pyramidal neurons of the forebrain resulted in a reduction of VGLUT1 levels in the striatum and an increase in striatal dopamine levels.

Biochemically, I have shown that a functional consequence of FBXO7 knockdown in cultured cells was a reduction in proteasomal activity similar to the one seen upon knockdown of a proteasomal core subunit. Taken together, I have established mouse models that recapitulate features seen in PARK15 patients where the symptoms are distinguished and restricted to neuronal subtypes involved in the disease. My research revealed FBXO7 as a crucial player in cellular health and pointed to a role in proteasomal regulation. 


\subsection{FBX07 as a proteasomal regulator}

Impairment in proteasomal function has long been thought to be one of the mechanisms underlying Parkinson's disease (McNaught et al., 2001). FBXO7's relationship with the proteasomal interactor PI31 prompted us to further investigate FBXO7's role in proteasomal regulation. As David Brockelt established PSMA2, a proteasomal core subunit, as a novel FBXO7 interaction partner, I first showed that knockdown of PSMA2 in HEK293T cells led to about $20 \%$ reduction in proteasome activity. I then showed a similar reduction of proteasomal activity upon $\mathrm{FBXO} 7$ knockdown. As PI31 closely interacts with FBXO7 and has been reported to regulate the proteasome, I knocked it down in a similar manner, but saw no change in proteasomal activity. The latter is not surprising given that other groups have previously not been able to recapitulate PI31's inhibitory or activating effects on the mammalian proteasome in vivo (Li et al., 2014; Zaiss et al., 2002). It is possible that $\mathrm{PI} 31$ only acts as a regulator of spatially confined proteasomes, or that it depends heavily on other proteins, so that its knockdown in itself is not enough to show an effect on global proteasome function (Li et al., 2014). However, our results establish that FBXO7 itself has a profound effect on proteasome function. It is therefore plausible that if PI31 acts on the proteasome in vivo, it could be mediated through FBXO7. In this context, I could show that PI31 associated with PSMA2 through FBXO7 in a coimmunoprecipitation assay. It is of course possible that PI31 simultaneously interacts with other proteasomal subunits, but in our assay it conveyed no modulatory effects on its own. FBXO7 and PI31 have been shown to interact tightly, and in light of our finding that $\mathrm{PI} 31$ in heavily downregulated in the $\mathrm{FBXO7}$ knockout mouse brain, it is tempting to speculate that FBXO7 stabilizes PI31 protein levels. This stabilization is likely to occur primarily through a direct interaction rather than a ubiquitination as PI31 and FBXO7 dimerize in vitro (Kirk et al., 2008). The same study suggested that PI31 could regulate FBXO7 by interfering with its homodimerization. Other SCF-type E3 ligases require homodimerization to execute their ligase function. For example, disruption of Cdc4-SCF dimerization leads to a decrease in the ubiquitin chain elongation by this ligase (Orlicky et al., 2003; Tang et al., 2007). As we did not observe a change in proteasomal activity upon $\mathrm{PI} 31$ knockdown, this mechanism is probably not important for FBXO7's function on the proteasome. In addition, FBXO7 can form multimers and is thus able to both dimerize with PI31 and itself (Shang et al., 2014). It has previously been suggested that these two proteins are present in different cellular compartments (Kirk et al., 2008), but our pulldown assays show that at least a subset are localized to the same proteasomes, so this cannot explain the lack of effect upon PI31 knockdown. 
David Brockelt could also show that FBXO7 likely ubiquitinated PSMA2 through a K63linked ubiquitination mechanism. I could further show that knockdown of PI31 leads to an increase in FBXO7-mediated ubiquitination of PSMA2. We hypothesized that FBXO7mediated ubiquitination enhances proteasomal assembly by functional modification of PSMA2. PI31 has previously been thought to mediate proteasomal assembly in vitro (Cho-Park and Steller, 2013). Here, PI31 binds to and inhibits the individual 20 S core subunits, but upon close proximity of the 19S cap subunit, PI31 then binds to $19 \mathrm{~S}$ chaperones and facilitate binding of the cap to the core. This hypothesis could explain why PI31 functions as a proteasomal activator in Drosophila melanogaster (Bader et al., 2011). Its interaction partner there, nutcracker, is however only a partial homologue of FBXO7 and might not have a similar function to the mammalian FBXO7. We found the $\mathrm{Ubl}$ domain of $\mathrm{FBXO} 7$ to be essential for its interaction with PSMA2 and this domain is not shared by PI31. Therefore, it is possible that in mammalian cells FBXO7 has a more important role in proteasomal regulation than $\mathrm{PI} 31$. Taken together, we have established a role for FBXO7 as an important proteasomal regulator, which is independent of its interaction with $\mathrm{PI} 31$.

In my master thesis, I showed that knockdown of FBXO7 led to mitochondrial fragmentation in hippocampal neurons. Given FBXO7's interaction with parkin and PINK1, there are indications that $\mathrm{FBXO}$ plays a role in mitochondrial quality control (Burchell et al., 2013). Mitochondria and proteasomes are interdependent cellular control organelles and a dysfunction in one system might trigger dysfunction in the other. Mitochondrial defects and an increase in reactive oxygen species have been found to result in an increase in proteasomal disassembly (Livnat-Levanon et al., 2014). Conversely, proteasomal inhibition can impair mitochondrial homeostasis and turnover (Sullivan et al., 2004). In our mouse models, we saw no indication of increased oxidative stress, but further investigation of the mitochondrial health in $\mathrm{FBXO} 7$ knockout neurons is required. It will inevitably be difficult to prove which cellular mechanism is the chicken and which is the egg in this case, but FBXO7 emerges as a crucial player in mitochondrial as well as proteasomal control and may provide a link between these heavily interconnected cellular compartments.

\subsection{Systemic loss of FBXO7 is detrimental to the organism}

Through the generation of an FBXO7 conventional knockout mouse, I have recapitulated the features of early onset Parkinson's disease in a rodent model. I could show that the homozygous knockout mice die prematurely and are characterized by a loss of motor 
function. Phenotypic characterization revealed a marked reduction in body and brain size in addition to well-pronounced muscle weakness. It has previously been suggested that FBXO7 is involved in hematopoesis and a partial knockout mouse was shown to have a reduced red blood cell count (Randle et al., 2015). It is therefore conceivable that our knockout mouse also suffers from anemia and this could contribute to the premature death. In addition, FBXO7's ubiquitous expression suggests that it is involved in cellular functions throughout the body. Mitochondrial and proteasomal function as well as cell cycle regulation are not neuron-specific processes and dysfunction in either pathway could contribute to cell dysfunction in multiple organs. The FBXO7 knockout pups usually die around P21, which is the weaning time. Dysphagia has been shown to be a common comorbidity in PARK15 patients (Yalcin-Cakmakli et al., 2014), and the general muscle weakness in the homozygous animals could make it hard to tackle the adjustment to solid food. The cause of death for the FBXO7 knockout mice is therefore hard to determine and could be caused by defects outside of, as well as inside the nervous system.

The muscle weakness and the kyphosis seen in the FBXO7 knockout mice are strongly reminiscent of the phenotype seen in mice, in which FBXO7 is knocked out in myelinating cells (data not shown). It has previously been shown that FBXO7 is strongly expressed in white matter (Zhang et al., 2014) and these symptoms suggest that glial involvement is important to the phenotype of the conventional knockout mouse. Also in a PARK15 patient, white matter lesions were observed upon brain scans (Lohmann et al., 2015). In addition to possible glial involvement and denervation of muscles, the muscle fibers themselves are heavily dependent upon an intact mitochondrial and proteasomal machinery. Although proteasome inhibition by MG132 has been shown to protect skeletal muscles from atrophy (Caron et al., 2011; Jamart et al., 2011), this could also be attributed to a disturbance in, for instance, the inflammatory response (Kitajima et al., 2014). Upon conditional knockdown of the proteasomal subunit Rpt3 in motor neurons or muscle cells, a progressive motor deterioration or a reduction in muscle strength and premature death was observed respectively (Kitajima et al., 2014; Tashiro et al., 2012). Additionally, it has been shown that expression of the mitochondrial fission machinery in muscle leads to atrophy and fission inhibition can have a corresponding protective function (Romanello et al., 2010). In my master thesis, I could show fragmented mitochondria in hippocampal and cerebellar granule cultured neurons, and it is therefore feasible that this phenotype also occurs in muscle fibers, contributing to the weakness seen in the FBXO7 -/- mice. The slight hyperactivity and stereotypic jumping seen in the P18 FBXO7 -/- mice are similar to the phenotype seen in NEX-Cre;fl/fl mice at this age. This suggests that also in the systemic knockout, pyramidal neurons are heavily affected. 
In P18 FBXO7 knockout mice, we did not observe a change in striatal dopamine levels. This could either be because it is too early for dopaminergic defects to show, or there are compensatory mechanisms at play. Given that the two month-old TH-Cre;fl/fl mice showed a reduction in striatal dopamine, while NEX-Cre;fl/fl animals at the same age show an increase, it could also be that these effects equalized one another in the young FBXO7 knockout mice.

We also saw increased apoptosis in cortical neurons of the conventional knockout. This corresponded nicely with patient data showing general brain atrophy in some PARK15 patients (Paisan-Ruiz et al., 2010). Although proteasome dysfunction might not be the only mechanism by which loss of FBXO7 contributes to apoptosis, it is interesting to note that severe neurodegeneration was observed upon selective disruption of $26 \mathrm{~S}$ proteasome function in mouse forebrain or tyrosine hydroxylase positive neurons (Bedford et al., 2008). I could not show any substantial loss of either TH-positive cell bodies in the substantia nigra or terminal markers in the striatum of knockout mice at P18. Since no pathological data is available from the PARK15 patients, it is hard to say whether they suffer from a specific loss of dopaminergic neurons. The loss of presynaptic dopaminergic function as seen in the CIT-SPECT of two patients does not necessarily indicate a loss of cell bodies (Di Fonzo et al., 2009; Hanagasi et al., 2007).

Taken together, our conventional FBXO7 knockout mouse model replicates cardinal features of the PARK15 patients, but is not sufficient to reveal neuron-specific pathology.

\subsection{Modeling the pyramidal syndrome seen in PARK15 patients}

The PARK15 syndrome is characterized by both pyramidal and Parkinsonian symptoms. In most patients the Parkinsonian symptoms dominate, but in the first described Iranian kindred all patients show pyramidal signs as their first symptoms (Shojaee et al., 2008). Only the most severely affected individuals show Parkinsonian symptoms like tremor and rigidity. Pyramidal signs stem from lesions in the upper motor neurons and comprise among others hyperreflexia, Babinskis sign, spasticity and loss of motor control. With our research, we have modeled these symptoms by creating a conditional NEX-Cre FBXO7 knockout mouse (NEX-Cre;fl/fl), in which FBXO7 was mainly deleted in the excitatory neurons of the cortex and hippocampus. With a wide array of behavioral tests, I have shown that this model displays loss of motor control as well as a multitude of stereotypic behaviors. 
Upon initial contact with the homozygous NEX-Cre;fl/fl mice, they started jumping and biting down on the grid in a spastic manner. Their muscles tensed so much; it was hard to keep them still. This was accompanied by a strong clasping in all limbs. In addition, it was clear that on both the rotarod and the balance beam the mice had problems moving their limbs in fluent motions. They moved in a jerky fashion, had problems holding and gripping on to surfaces. This is strongly reminiscent of the abnormal reflexes and spasticity seen in PARK15 patients and indicates a loss of function in upper motor neurons. Their spasticity eventually made it difficult to properly grab and eat food, while their hyperactivity probably caused increased metabolic demand. This starts showing from two months on as the mice did not gain any weight and became progressively weaker. We chose to terminate experiments at four months of age as the mice showed signs of pain.

Other prominent features of the NEX-Cre;fl/fl mice were hyperactivity and stereotypic behavior. Stereotypic behavior can be measured in rodents in a multitude of tests and here I could show that these mice showed a high degree of circling and scratching, but no increase in marble burying, grooming or climbing. In the Y-maze, the mice kept a strict pattern of arm entry and in a novel object recognition test the mice sniffed one object, then the other, then back to the first, and kept going in this pattern for several minutes. In the light avoidance test, I could show that the mice stayed in either compartment and changed location less frequently than their controls. This was also reflected in the elevated plus maze, where the NEX-Cre;fl/fl animals would stay in the open arm for a prolonged period of time. Taken together, these results suggest perseverance in locomotor behavior. The mice had difficulties ending a behavior once it had been initiated. This could also explain why grooming is not exaggerated, as this behavior might transition to higher scratching frequency. The same is true for the marble burying where the sniffing and circling pattern would overcome the burying behavior. These symptoms of strong stereotypies, perseverative locomotor behavior and hyperactivity are strongly reminiscent of the symptoms seen in a knockout mouse model of the dopamine transporter (DAT). These DAT knockout mice also present with hyperactivity and perseverative locomotor behavior (Fox et al., 2013). In these mice, the symptoms are seen in connection with increased and prolonged extracellular levels of dopamine in the striatum due to dysfunctional dopamine reuptake (Giros et al., 1996). Interestingly, a recently described PARK15 patient showed obsessive-compulsive traits (Conedera et al., 2016). In addition, several PARK15 patients present with non-specified cognitive dysfunction (Table A1).

The stereotypic behavioral pattern prompted me to investigate the level of dopamine in the striatum of the NEX-Cre;fl/fl animals, and we could indeed show that the dopamine levels were significantly higher than in the striatum of control mice. This was accompanied 
by a slight up-regulation of the dopamine transporter, excluding re-uptake malfunction as a possible cause. As to how the glutamatergic afferents from the cortex influence dopaminergic output in the striatum is still under debate. Some studies have shown that extracellular glutamate can inhibit dopamine release in the striatum (Avshalumov et al., 2003; Zhang and Sulzer, 2003). Interestingly, I found a decrease in the vesicular glutamate transporter 1 (VGLUT1) transporter in the striatum, but not in cortex or cerebellum. As we saw increased apoptosis in the conventional knockout, we cannot rule out that cell death of glutamatergic neurons in the cortex contributes to the reduction in excitatory innervations in the striatum. However, my histological analysis shows no gross morphological disturbance in the NEX-Cre;fl/fl cortex.

On this basis, we hypothesized that the excitatory neurons in the cortex failed to release glutamate causing a lifted inhibition on the dopaminergic terminals and an increased dopaminergic tone in the striatum. As this does not seem to be caused by cell loss, we initiated a collaboration with Dr. Jeong-Seop Rhee and he conducted electrophysiological experiments on autaptic excitatory hippocampal neurons from FBXO7 knockout and control mice. In these experiments, he could show that the excitatory post-synaptic current (EPSC) was reduced along with a reduction in the readily releasable vesicle pool and decrease in release probability (data not shown). Interestingly, he could also show that the miniature EPSC frequency was decreased, while the amplitude remained normal. This electrophysiological phenotype was also shown in a mouse model with impaired glutamatergic transmission, the ProSAP1/Shank2-/- mice (Schmeisser et al., 2012). These mice, like ours, present with hyperactivity and stereotypies, further strengthening our hypothesis that the NEX-Cre;fl/fl mice might suffer from an decrease in striatal glutamate transmission.

\subsection{Loss of FBXO7 in catecholaminergic neurons}

As Parkinsonian-Pyramidal Syndrome encompasses only a minor subset of PD spectrum disorders, we wanted to isolate the symptoms that are related to sporadic PD. We therefore created an FBXO7 knockout mouse restricted to catecholaminergic neurons using the tyrosine hydroxylase (TH) promoter (TH-Cre;fl/fl). I could show with a series of behavioral tests that these mice had normal motor characteristics at two months of age, but became progressively more challenged from six to twelve months. In the rotarod test, I showed that there was minor impairment already at six months. 
To further quantify motor disturbances in these mice, we initiated a collaboration with Camille Lancelin who determined, using DigiGait testing, that fine gait parameters were changed at eight months of age. Particularly interesting was the finding that both brake, stance and stride time decreased, in addition to shorter stride length and higher stride frequency. This is clearly reminiscent of an MPTP mouse model of PD that was tested with the same apparatus hinting at a similar functional loss in the two models (Amende et al., 2005).

The loss of fine motor control progressed into a decrease in ambulation as well as inability to perform the rotarod task at twelve months of age. This suggests a progressive loss of motor function starting with gait disturbances and slowness of movements leading to a decrease in ambulation. It is unlike what we show in the NEX-Cre;fl/fl model, where discoordination and spasticity are pronounced phenotypes. The TH-Cre;fl/fl mice hence modeled rather bradykinesia and rigidity characteristic for PARK15 and sporadic PD patients.

To monitor if the symptoms were correlated with a loss of dopaminergic innervation, I conducted stereological counting of neurons in the substantia nigra, but did not find any neuronal loss at either two or twelve months of age. However, when we measured the levels of dopamine in the striatum we found a significant down-regulation at twelve months of age as compared to control. Surprisingly, the levels were equally low at two months, a time point where no discernible motor symptoms were present. An explanation for this might be to find in PD patients themselves. It is estimated that symptoms of PD do not show until about $30 \%$ of dopaminergic neurons in the substantia nigra are lost (Fearnley and Lees, 1991; Greffard et al., 2006; Ma et al., 1997). Another study visualizing this phenomenon followed PD patients over 40 weeks (Fahn et al., 2004). Their motor symptoms were clearly getting worse while the dopamine level stayed the same. This suggests that onset and progression of motor symptoms are not linearly correlated with the loss of dopamine. The basal ganglia is a heavily interregulated system with many connections to other brain areas. Several compensatory mechanisms, both within and outside of the basal ganglia, have been suggested to account for the discrepancy in correlation between loss of dopamine and the emergence of motor symptoms (Bezard et al., 2003). Thus we hypothesize that in our TH-Cre;fl/fl model, a prolonged deficiency in dopaminergic tone in the striatum leads to gradual change of basal ganglia connectivity and progressive motor dysfunction. One indication of the change in striatal connectivity over time is the normal levels of DAT in the TH-Cre;fl/fl striatum at two months compared to the decrease seen at twelve months of age. 
In addition to the loss of motor function, I observed that TH-Cre;fl/fl mice were significantly overweight at six months of age. As dopamine is involved in several brain areas related to feeding behavior, it was reasonable to assume that the reduction in dopamine also affected the $\mathrm{TH}-\mathrm{Cre} ; \mathrm{fl} / \mathrm{fl}$ food consumption. Although dopamine is classically associated with reward behavior, I demonstrated that the sucrose preference of the TH-Cre;fl/fl mice was preserved. This was also the case for totally dopamine-deprived animals, suggesting that dopamine is not involved in the carbohydrate response to food (Cannon and Palmiter, 2003). Olfaction also did not seem to be changed in the TH-Cre;fl/fl mice, indicating that loss of smell does not account for the abnormal feeding behavior. Normal eating behavior evokes dopamine release in the striatum, which can contribute to satiety feeling. This has been hypothesized to be partially independent of eating itself and can be triggered directly by nutrient sensing in the gut (de Araujo et al., 2012). Dopamine deficiency in both acute and genetic paradigms has been shown to inhibit feeding in mice (Sotak et al., 2005). However, a reduction in dopamine can lead to increased feeding behavior, as it is associated with obesity in rats (Geiger et al., 2009; Rada et al., 2010). In the TH-Cre;fl/fl mice, I observed that they have been eating more from two to three months of age. This was not quantified; as I did not single house them for the observation period. However, at six months most mice were significantly heavier than their controls suggesting that a failure of dopamine release upon eating caused increased feeding behavior. At twelve months some animals were still heavier than the controls, while some were skinnier. It is possible that beneath a certain threshold, dopamine release is not sufficient to sustain feeding behavior and aphagia sets in. This needs further exploration, as I did not correlate weight with dopamine content in the striatum.

I observed that several animals got infections and lost weight after six months of age. This could be an indirect consequence of the previously mentioned obesity. Obesity in rodents and humans are correlated with a low-grade chronic inflammation in the adipose tissue and a heightened susceptibility to infections (Falagas and Kompoti, 2006).

As the TH-promoter is expressed in all catecholaminergic neurons, we know that the noradrenergic and adrenergic cells are also affected. Noradrenergic neurons in the brain are known to be involved in Parkinson's disease, and could contribute to motor symptoms (Delaville et al., 2011). Specifically, noradrenergic cells in the locus coeruleus (LC) are shown to be lost in sporadic PD patients, to an even greater extent than neurons in the substantia nigra (SN) (Zarow et al., 2003). It has also been proposed that LC loss might precede loss in SN (Braak and Del Tredici, 2008). The dopamine $\beta$-hydroxylase knockout mice that lack noradrenalin and adrenalin altogether, show severe motor symptoms, which can be attributed to the loss of noradrenalin centrally, not peripherally 
(Rommelfanger et al., 2007). In this study, Rommelfanger et al. also showed that MPTP treatment does not exacerbate motor symptoms in these mice, although it triggers an acute loss of $80 \%$ of the striatal dopamine. Taken together, the evidence so far suggests that a loss of both noradrenalin and dopamine in the brain of Parkinson's patients leads to the characteristic motor symptoms. We have not investigated the noradrenergic involvement in the brain of our $\mathrm{TH}-\mathrm{Cre}$;fl/fl mice, but given that loss of FBXO7 potentially triggers dysfunction in all neurons investigated, it is conceivable to assume that noradrenergic neurons also are affected. As recent treatment strategies explore therapeutics targeting both these systems (Espay et al., 2014), it will be interesting to see how they are affected in our mice. The TH-Cre;fl/fl model might provide an excellent opportunity to investigate the interplay between these systems, and test therapeutic agents.

Another striking finding in all mouse models is the presence of reactive astrogliosis. Most evidence from PD patients suggests a role for microglia in chronic brain inflammation where they contribute to dopaminergic neuron degeneration in the midbrain (Przedborski, 2007). However, in the TH-Cre;fl/fl mice, I found no up-regulation of either normal or activated microglia as Iba1 and Mac3 staining appeared comparable to control. Astrocytes, however, are upregulated in all models, despite a clear loss of neurons. There is an on-going debate whether immune response is damaging or actually protective for neurons. Astrogliosis accompanies dead or damaged cells in both the neurotoxic (MPTP) (Kato et al., 2003) and the ATP13a2 knockout mouse models (Kett et al., 2015), while astrocytes are also suggested to serve a protective role in PD and precede neuronal loss (Saura et al., 2003; Sortwell et al., 2000). This is one possible explanation for the limited neuronal loss we saw in our conditional FBXO7 knockout mice.

Another aspect of the immune response is FBXO7's involvement in NF- $\kappa B$ signaling. It has been shown that FBXO7 can ubiquitinate cIAP1 and TRAF2 as to lower the NF- $\mathrm{KB}$ response (Kuiken et al., 2012). With FBXO7 gone, it is possible that NF- $\kappa B$ signaling is increased in these mice. In glia, NF- $\mathrm{KB}$ is heavily involved in immune responses, while in neurons an increase in NF- $\mathrm{KB}$ signaling has been hypothesized to have neuroprotective effects against insults such as oxidative stress (Mattson et al., 1997). Hence, the slight increase in apoptosis in the conventional knockouts might be partially due to increased $\mathrm{NF}-\kappa \mathrm{B}$ signaling in microglia, while the conditional FBXO7 knockout mice might benefit from its neuroprotective role in neurons. This an interesting line of investigation that should be continued to shed further light on the immune system involvement in PARK15 patients. 
As Lewy bodies are a cardinal pathological feature of PD, David Brockelt stained midbrain sections from conventional FBXO7-/- mice for $\alpha$-synuclein, but found no inclusions (data not shown). The lack of protein aggregates in all FBXO7-/- mouse brains correlates with the absence of such aggregates in brains of typical juvenile Parkinsonism patients (Poulopoulos et al., 2012) and is therefore not surprising.

\subsection{Loss of FBXO7 causes neuronal dysfunction}

Loss of FBXO7 in both conditional knockouts caused neuronal dysfunction. In light of the electrophysiological findings in the conventional knockout mice, it is tempting to speculate that synaptic dysfunction is responsible. The failure of release seen in excitatory neurons might be transferable to dopaminergic neurons. The release mechanism of dopamine is less characterized than the excitatory/inhibitory release, but many of the main features are shared. A question that definitely warrants further investigation is whether we are looking at a reduced synapse number or a decreased function of individual synapses. Hence, morphological analyses of FBXO7-/- neurons will be important future experiments.

E3 ubiquitin ligases have a wide variety of targets and each ligase can be involved in multiple pathways, as is the case for FBXO7. The cause for the apparent neuronal dysfunction seen in the FBXO7 knockout animals is hence difficult to elucidate. We have in this and previous studies shown that $\mathrm{FBXO} 7$ is involved in proteasomal regulation. $\mathrm{A}$ tight regulation of protein levels is crucial to all cells, and dysfunctional proteasomes can lead to an accumulation of damaged proteins. This might be more harmful in neurons as they are post-mitotic cells with a highly compartmentalized structure and high metabolic demand. It has for example been shown that neurons are more affected than astrocytes by proteasome inhibition in cultured rat cells (Dasuri et al., 2010).

Proteasomes are present in the synapse and involved in the precise control of protein composition required for optimal synapse formation and function. Acute proteasomal inhibition at the synapse can lead to an increase in the readily releasable vesicle pool and cause a strengthening of synapses (Willeumier et al., 2006). The consequence of chronically reduced proteasomal activity however is not well studied. Interestingly, the proteasome has been implicated in proper axonal outgrowth through the APC complex, which is one of the first described E3 ligases (Konishi et al., 2004; Stegmuller et al., 2006). It is therefore conceivable that impairment of proteasomal function through development can contribute to improper synapse formation. 
In addition, FBXO7 has been implicated in mitochondrial quality control. Mitochondria are crucial for synaptic health, shown for instance by screens conducted in Drosophila melanogaster, in which mutations in mitochondrial proteins lead to synaptic dysfunction (Verstreken et al., 2005). Mitochondria mainly work at the synapse to regulate calcium levels (Rizzuto et al., 2004) or generate ATP, the latter probably most important for synaptic function (Verstreken et al., 2005).

In terms of proper synapse formation, the light chain of the microtubule associated protein 1B (MAP1B-LC1) is another interesting interaction partner of FBXO7, identified by David Brockelt (data not shown). This protein has been implicated in axonal growth and guidance (Gonzalez-Billault et al., 2001; Takei et al., 2000) and can be modified by ubiquitination (Yonashiro et al., 2012). This interaction might be interesting to investigate in light of both axon outgrowth and proper synapse formation, but also in terms of axonal transport of for instance mitochondria.

If FBXO7 causes changes in cellular functions common to all cells, it is an interesting question to answer why we observe such a specific symptom pattern in the PARK15 patients. One important point is that $\mathrm{FBXO7}$ has shown a particularly strong expression in the pyramidal neurons (Zhao et al., 2013) of the cerebral cortex. In addition we know that the dopaminergic and noradrenergic neurons are considered vulnerable due to their high metabolic load. Therefore these cells types might be especially compromised by loss of FBXO7 and contribute to the pyramidal and Parkinsonian symptoms we see in PARK15 patients. The different expression of the symptoms in individual families might differ due to genotypic background and environmental factors. In addition, it is possible that the different mutations affect different aspects of $\mathrm{FBXO} 7$ function on top of rendering it destabilized. 


\section{Conclusions and perspectives}

In this study I have characterized three FBXO7 mouse models. At the systemic level, loss of FBXO7 is detrimental to mice, as they show growth retardation, weakness and premature death. When FBXO7 is absent from forebrain neurons and hippocampus, I find a behavioral phenotype consisting of severe spasticity, hyperactivity and perseverative locomotor behavior. In contrast, loss of FBXO7 from catecholaminergic neurons leads to a progressive loss of fine motor control that causes reduced ambulation. While there is a significant increase in apoptosis in the cortex of the FBXO7-/- mice, there is no obvious neuronal loss in either of the conditional FBXO7 lines. In the forebrain knockout, I show an increase in dopamine in the striatum associated with a down-regulation in the vesicular glutamate transporter level. In the chatecholaminergic knockout however, I find a decrease in the dopamine level in the striatum. Electrophysiological measurements show a decrease in excitatory post-synaptic currents in neurons from the knockout mice. At the molecular level, I also show that FBXO7 is involved in proteasomal regulation, while I have previously shown that it might have an impact on mitochondrial integrity. Based on these findings, I conclude that loss of $\mathrm{FBXO} 7$ in murine neurons leads to cellular dysfunction not necessarily accompanied by neuronal loss in vivo. The mechanisms underlying this dysfunction remain to be explored, but dysfunction of the proteasomal system, as well as mitochondrial disturbances are conceivable. My work laid the foundation for further investigation of the role of FBXO7 in synaptic function, in proteasomal regulation and mitochondrial integrity. Also, my characterization of two conditional FBXO7 mouse models will prove useful to study pathways involved in Parkinsonian-Pyramidal Syndrome and explore potential therapeutic targets. 


\section{Bibliography}

Alam, Z.I., Daniel, S.E., Lees, A.J., Marsden, D.C., Jenner, P., and Halliwell, B. (1997). A generalised increase in protein carbonyls in the brain in Parkinson's but not incidental Lewy body disease. J Neurochem 69, 1326-1329.

Alt, J.R., Gladden, A.B., and Diehl, J.A. (2002). p21(Cip1) promotes cyclin D1 nuclear accumulation via direct inhibition of nuclear export. J Biol Chem 277, 8517-8523.

Alvarez-Fischer, D., Henze, C., Strenzke, C., Westrich, J., Ferger, B., Hoglinger, G.U., Oertel, W.H., and Hartmann, A. (2008). Characterization of the striatal 6-OHDA model of Parkinson's disease in wild type and alpha-synuclein-deleted mice. Exp Neurol 210, $182-$ 193.

Amende, I., Kale, A., McCue, S., Glazier, S., Morgan, J.P., and Hampton, T.G. (2005). Gait dynamics in mouse models of Parkinson's disease and Huntington's disease. J Neuroeng Rehabil 2, 20.

Avshalumov, M.V., Chen, B.T., Marshall, S.P., Pena, D.M., and Rice, M.E. (2003). Glutamate-dependent inhibition of dopamine release in striatum is mediated by a new diffusible messenger, H2O2. J Neurosci 23, 2744-2750.

Bader, M., Benjamin, S., Wapinski, O.L., Smith, D.M., Goldberg, A.L., and Steller, H. (2011). A conserved $\mathrm{F}$ box regulatory complex controls proteasome activity in Drosophila. Cell 145, 371-382.

Bassal, S., Nomura, N., Venter, D., Brand, K., McKay, M.J., and van der Spek, P.J. (2001). Characterization of a novel human cell-cycle-regulated homologue of Drosophila dlg1. Genomics 77, 5-7.

Bedford, L., Hay, D., Devoy, A., Paine, S., Powe, D.G., Seth, R., Gray, T., Topham, I., Fone, K., Rezvani, N., et al. (2008). Depletion of $26 \mathrm{~S}$ proteasomes in mouse brain neurons causes neurodegeneration and Lewy-like inclusions resembling human pale bodies. J Neurosci 28, 8189-8198.

Belzung, C., Misslin, R., Vogel, E., Dodd, R.H., and Chapouthier, G. (1987). Anxiogenic effects of methyl-beta-carboline-3-carboxylate in a light/dark choice situation. Pharmacol Biochem Behav 28, 29-33.

Bernheimer, H., Birkmayer, W., Hornykiewicz, O., Jellinger, K., and Seitelberger, F. (1973). Brain dopamine and the syndromes of Parkinson and Huntington. Clinical, morphological and neurochemical correlations. J Neurol Sci 20, 415-455.

Besche, H.C., and Goldberg, A.L. (2012). Affinity purification of mammalian 26S proteasomes using an ubiquitin-like domain. Methods Mol Biol 832, 423-432.

Bezard, E., Gross, C.E., and Brotchie, J.M. (2003). Presymptomatic compensation in Parkinson's disease is not dopamine-mediated. Trends Neurosci 26, 215-221.

Bilimoria, P.M., and Bonni, A. (2008). Cultures of cerebellar granule neurons. CSH Protoc 2008, pdb prot5107.

Bindoff, L.A., Birch-Machin, M.A., Cartlidge, N.E., Parker, W.D., Jr., and Turnbull, D.M. (1991). Respiratory chain abnormalities in skeletal muscle from patients with Parkinson's disease. J Neurol Sci 104, 203-208. 
Biondi, O., Branchu, J., Sanchez, G., Lancelin, C., Deforges, S., Lopes, P., Pariset, C., Lecolle, S., Cote, J., Chanoine, C., et al. (2010). In vivo NMDA receptor activation accelerates motor unit maturation, protects spinal motor neurons, and enhances SMN2 gene expression in severe spinal muscular atrophy mice. J Neurosci 30, 11288-11299.

Blesa, J., and Przedborski, S. (2014). Parkinson's disease: animal models and dopaminergic cell vulnerability. Front Neuroanat 8, 155.

Bochtler, M., Ditzel, L., Groll, M., Hartmann, C., and Huber, R. (1999). The proteasome. Annu Rev Biophys Biomol Struct 28, 295-317.

Bonifacino, J.S., Dell'Angelica, E.C., and Springer, T.A. (2001). Immunoprecipitation. Curr Protoc Immunol Chapter 8, Unit 83.

Bonifati, V., Rizzu, P., Squitieri, F., Krieger, E., Vanacore, N., van Swieten, J.C., Brice, A., van Duijn, C.M., Oostra, B., Meco, G., et al. (2003). DJ-1( PARK7), a novel gene for autosomal recessive, early onset parkinsonism. Neurol Sci 24, 159-160.

Bousquet-Dubouch, M.P., Baudelet, E., Guerin, F., Matondo, M., Uttenweiler-Joseph, S., Burlet-Schiltz, O., and Monsarrat, B. (2009). Affinity purification strategy to capture human endogenous proteasome complexes diversity and to identify proteasomeinteracting proteins. Mol Cell Proteomics 8, 1150-1164.

Bove, J., Zhou, C., Jackson-Lewis, V., Taylor, J., Chu, Y., Rideout, H.J., Wu, D.C., Kordower, J.H., Petrucelli, L., and Przedborski, S. (2006). Proteasome inhibition and Parkinson's disease modeling. Ann Neurol 60, 260-264.

Braak, H., and Del Tredici, K. (2008). Invited Article: Nervous system pathology in sporadic Parkinson disease. Neurology 70, 1916-1925.

Bradford, M.M. (1976). A rapid and sensitive method for the quantitation of microgram quantities of protein utilizing the principle of protein-dye binding. Anal Biochem 72, 248254.

Brice, A. (2005). Genetics of Parkinson's disease: LRRK2 on the rise. Brain 128, $2760-$ 2762.

Brummelkamp, T.R., Bernards, R., and Agami, R. (2002). A system for stable expression of short interfering RNAs in mammalian cells. Science 296, 550-553.

Burchell, V.S., Nelson, D.E., Sanchez-Martinez, A., Delgado-Camprubi, M., Ivatt, R.M., Pogson, J.H., Randle, S.J., Wray, S., Lewis, P.A., Houlden, H., et al. (2013). The Parkinson's disease-linked proteins Fbxo7 and Parkin interact to mediate mitophagy. Nat Neurosci 16, 1257-1265.

Cannon, C.M., and Palmiter, R.D. (2003). Reward without dopamine. J Neurosci 23, 10827-10831.

Cardozo, T., and Pagano, M. (2004). The SCF ubiquitin ligase: insights into a molecular machine. Nat Rev Mol Cell Biol 5, 739-751.

Caron, A.Z., Haroun, S., Leblanc, E., Trensz, F., Guindi, C., Amrani, A., and Grenier, G. (2011). The proteasome inhibitor MG132 reduces immobilization-induced skeletal muscle atrophy in mice. BMC Musculoskelet Disord 12, 185.

Cenciarelli, C., Chiaur, D.S., Guardavaccaro, D., Parks, W., Vidal, M., and Pagano, M. (1999). Identification of a family of human F-box proteins. Curr Biol 9, 1177-1179. 
Chang, Y.F., Cheng, C.M., Chang, L.K., Jong, Y.J., and Yuo, C.Y. (2006). The F-box protein Fbxo7 interacts with human inhibitor of apoptosis protein clAP1 and promotes clAP1 ubiquitination. Biochem Biophys Res Commun 342, 1022-1026.

Chen, C.M., Chen, I.C., Huang, Y.C., Juan, H.F., Chen, Y.L., Chen, Y.C., Lin, C.H., Lee, L.C., Lee, C.M., Lee-Chen, G.J., et al. (2014). FBXO7 Y52C polymorphism as a potential protective factor in Parkinson's disease. PLoS ONE 9, e101392.

Chen, G., and Goeddel, D.V. (2002). TNF-R1 signaling: a beautiful pathway. Science 296, 1634-1635.

Cheng, F., Vivacqua, G., and Yu, S. (2011). The role of alpha-synuclein in neurotransmission and synaptic plasticity. J Chem Neuroanat 42, 242-248.

Cheng, M., Olivier, P., Diehl, J.A., Fero, M., Roussel, M.F., Roberts, J.M., and Sherr, C.J. (1999). The p21(Cip1) and p27(Kip1) CDK /'inhibitors/' are essential activators of cyclin D-dependent kinases in murine fibroblasts. EMBO J 18, 1571-1583.

Chesselet, M.F., Richter, F., Zhu, C., Magen, I., Watson, M.B., and Subramaniam, S.R. (2012). A progressive mouse model of Parkinson's disease: the Thy1-aSyn ("Line 61") mice. Neurotherapeutics 9, 297-314.

Chiu, A.W., Huang, Y.L., Huan, S.K., Wang, Y.C., Ju, J.P., Chen, M.F., and Chou, C.K. (2002). Potential molecular marker for detecting transitional cell carcinoma. Urology 60, 181-185.

Chomczynski, P. (1993). A reagent for the single-step simultaneous isolation of RNA, DNA and proteins from cell and tissue samples. Biotechniques 15, 532-534, 536-537.

Cho-Park, P.F., and Steller, H. (2013). Proteasome regulation by ADP-ribosylation. Cell 153, 614-627.

Chung, C.Y., Koprich, J.B., Siddiqi, H., and Isacson, O. (2009). Dynamic changes in presynaptic and axonal transport proteins combined with striatal neuroinflammation precede dopaminergic neuronal loss in a rat model of AAV alpha-synucleinopathy. $\mathrm{J}$ Neurosci 29, 3365-3373.

Chu-Ping, M., Slaughter, C.A., and DeMartino, G.N. (1992). Purification and characterization of a protein inhibitor of the $20 \mathrm{~S}$ proteasome (macropain). Biochim Biophys Acta 1119, 303-311.

Conedera, S., Apaydin, H., Li, Y., Yoshino, H., Ikeda, A., Matsushima, T., Funayama, M., Nishioka, K., and Hattori, N. (2016). FBXO7 mutations in Parkinson's disease and multiple system atrophy. Neurobiol Aging.

Crawley, J.N. (2003). Behavioral phenotyping of rodents. Comp Med 53, 140-146.

Dasuri, K., Ebenezer, P.J., Zhang, L., Fernandez-Kim, S.O., Uranga, R.M., Gavilan, E., Di Blasio, A., and Keller, J.N. (2010). Selective vulnerability of neurons to acute toxicity after proteasome inhibitor treatment: implications for oxidative stress and insolubility of newly synthesized proteins. Free Radic Biol Med 49, 1290-1297.

Davis, G.C., Williams, A.C., Markey, S.P., Ebert, M.H., Caine, E.D., Reichert, C.M., and Kopin, I.J. (1979). Chronic Parkinsonism secondary to intravenous injection of meperidine analogues. Psychiatry Res 1, 249-254.

de Araujo, I.E., Ferreira, J.G., Tellez, L.A., Ren, X., and Yeckel, C.W. (2012). The gutbrain dopamine axis: a regulatory system for caloric intake. Physiol Behav 106, 394-399. 
Decressac, M., Mattsson, B., Lundblad, M., Weikop, P., and Bjorklund, A. (2012). Progressive neurodegenerative and behavioural changes induced by AAV-mediated overexpression of alpha-synuclein in midbrain dopamine neurons. Neurobiol Dis 45, 939953.

Delaville, C., Deurwaerdere, P.D., and Benazzouz, A. (2011). Noradrenaline and Parkinson's disease. Front Syst Neurosci 5, 31.

Dere, E., Dahm, L., Lu, D., Hammerschmidt, K., Ju, A., Tantra, M., Kastner, A., Chowdhury, K., and Ehrenreich, H. (2014). Heterozygous ambra1 deficiency in mice: a genetic trait with autism-like behavior restricted to the female gender. Front Behav Neurosci 8, 181.

Deshaies, R.J., and Joazeiro, C.A. (2009). RING domain E3 ubiquitin ligases. Annu Rev Biochem 78, 399-434.

Di Fonzo, A., Dekker, M.C., Montagna, P., Baruzzi, A., Yonova, E.H., Correia Guedes, L., Szczerbinska, A., Zhao, T., Dubbel-Hulsman, L.O., Wouters, C.H., et al. (2009). FBXO7 mutations cause autosomal recessive, early-onset parkinsonian-pyramidal syndrome. Neurology 72, 240-245.

Ding, K., Shameer, K., Jouni, H., Masys, D.R., Jarvik, G.P., Kho, A.N., Ritchie, M.D., McCarty, C.A., Chute, C.G., Manolio, T.A., et al. (2012). Genetic Loci implicated in erythroid differentiation and cell cycle regulation are associated with red blood cell traits. Mayo Clin Proc 87, 461-474.

Donnan, G.A., Willis, G.L., Kaczmarczyk, S.J., and Rowe, P. (1987). Motor function in the 1-methyl-4-phenyl-1,2,3,6-tetrahydropyridine-treated mouse. J Neurol Sci 77, 185191.

Dorsey, E.R., Constantinescu, R., Thompson, J.P., Biglan, K.M., Holloway, R.G., Kieburtz, K., Marshall, F.J., Ravina, B.M., Schifitto, G., Siderowf, A., et al. (2007). Projected number of people with Parkinson disease in the most populous nations, 2005 through 2030. Neurology 68, 384-386.

Espay, A.J., LeWitt, P.A., and Kaufmann, H. (2014). Norepinephrine deficiency in Parkinson's disease: the case for noradrenergic enhancement. Mov Disord 29, 17101719.

Fahn, S., Oakes, D., Shoulson, I., Kieburtz, K., Rudolph, A., Lang, A., Olanow, C.W., Tanner, C., and Marek, K. (2004). Levodopa and the progression of Parkinson's disease. N Engl J Med 351, 2498-2508.

Falagas, M.E., and Kompoti, M. (2006). Obesity and infection. Lancet Infect Dis 6, 438446.

Fearnley, J.M., and Lees, A.J. (1991). Ageing and Parkinson's disease: substantia nigra regional selectivity. Brain 114 ( Pt 5), 2283-2301.

Fischer, D.L., Gombash, S.E., Kemp, C.J., Manfredsson, F.P., Polinski, N.K., Duffy, M.F., and Sortwell, C.E. (2016). Viral Vector-Based Modeling of Neurodegenerative Disorders: Parkinson's Disease. Methods Mol Biol 1382, 367-382.

Forno, L.S. (1996). Neuropathology of Parkinson's disease. J Neuropathol Exp Neurol $55,259-272$. 
Fox, M.A., Panessiti, M.G., Hall, F.S., Uhl, G.R., and Murphy, D.L. (2013). An evaluation of the serotonin system and perseverative, compulsive, stereotypical, and hyperactive behaviors in dopamine transporter (DAT) knockout mice. Psychopharmacology (Berl) 227, 685-695.

Furukawa, Y., Vigouroux, S., Wong, H., Guttman, M., Rajput, A.H., Ang, L., Briand, M., Kish, S.J., and Briand, Y. (2002). Brain proteasomal function in sporadic Parkinson's disease and related disorders. Ann Neurol 51, 779-782.

Gallagher, S., Winston, S.E., Fuller, S.A., and Hurrell, J.G. (2008). Immunoblotting and immunodetection. Curr Protoc Immunol Chapter 8, Unit 810.

Gegg, M.E., Cooper, J.M., Chau, K.Y., Rojo, M., Schapira, A.H., and Taanman, J.W. (2010). Mitofusin 1 and mitofusin 2 are ubiquitinated in a PINK1/parkin-dependent manner upon induction of mitophagy. Hum Mol Genet 19, 4861-4870.

Geiger, B.M., Haburcak, M., Avena, N.M., Moyer, M.C., Hoebel, B.G., and Pothos, E.N. (2009). Deficits of mesolimbic dopamine neurotransmission in rat dietary obesity. Neuroscience 159, 1193-1199.

Giros, B., Jaber, M., Jones, S.R., Wightman, R.M., and Caron, M.G. (1996). Hyperlocomotion and indifference to cocaine and amphetamine in mice lacking the dopamine transporter. Nature 379, 606-612.

Goebbels, S., Bormuth, I., Bode, U., Hermanson, O., Schwab, M.H., and Nave, K.A. (2006). Genetic targeting of principal neurons in neocortex and hippocampus of NEX-Cre mice. Genesis 44, 611-621.

Goldberg, M.S., Fleming, S.M., Palacino, J.J., Cepeda, C., Lam, H.A., Bhatnagar, A., Meloni, E.G., Wu, N., Ackerson, L.C., Klapstein, G.J., et al. (2003). Parkin-deficient mice exhibit nigrostriatal deficits but not loss of dopaminergic neurons. J Biol Chem 278, 43628-43635.

Gombash, S.E., Manfredsson, F.P., Kemp, C.J., Kuhn, N.C., Fleming, S.M., Egan, A.E., Grant, L.M., Ciucci, M.R., MacKeigan, J.P., and Sortwell, C.E. (2013). Morphological and behavioral impact of AAV2/5-mediated overexpression of human wildtype alpha-synuclein in the rat nigrostriatal system. PLoS ONE 8, e81426.

Gonzalez-Billault, C., Avila, J., and Caceres, A. (2001). Evidence for the role of MAP1B in axon formation. Mol Biol Cell 12, 2087-2098.

Graham, F.L., Smiley, J., Russell, W.C., and Nairn, R. (1977). Characteristics of a human cell line transformed by DNA from human adenovirus type 5 . J Gen Virol 36, 5974.

Greffard, S., Verny, M., Bonnet, A.M., Beinis, J.Y., Gallinari, C., Meaume, S., Piette, F., Hauw, J.J., and Duyckaerts, C. (2006). Motor score of the Unified Parkinson Disease Rating Scale as a good predictor of Lewy body-associated neuronal loss in the substantia nigra. Arch Neurol 63, 584-588.

Groll, M., Bochtler, M., Brandstetter, H., Clausen, T., and Huber, R. (2005). Molecular machines for protein degradation. Chembiochem 6, 222-256.

Gross-Bellard, M., Oudet, P., and Chambon, P. (1973). Isolation of high-molecularweight DNA from mammalian cells. Eur J Biochem 36, 32-38. 
Gruden, M.A., Davydova, T.V., Narkevich, V.B., Fomina, V.G., Wang, C., Kudrin, V.S., Morozova-Roche, L.A., and Sewell, R.D. (2014). Intranasal administration of alphasynuclein aggregates: a Parkinson's disease model with behavioral and neurochemical correlates. Behav Brain Res 263, 158-168.

Grunblatt, E., Zehetmayer, S., Jacob, C.P., Muller, T., Jost, W.H., and Riederer, P. (2010). Pilot study: peripheral biomarkers for diagnosing sporadic Parkinson's disease. J Neural Transm (Vienna) 117, 1387-1393.

Gunduz, A., Eken, A.G., Bilgic, B., Hanagasi, H.A., Bilguvar, K., Gunel, M., Basak, A.N., and Ertan, S. (2014). FBXO7-R498X mutation: Phenotypic variability from chorea to early onset parkinsonism within a family. Parkinsonism Relat Disord 20, 1253-1256.

Guyenet, S.J., Furrer, S.A., Damian, V.M., Baughan, T.D., La Spada, A.R., and Garden, G.A. (2010). A simple composite phenotype scoring system for evaluating mouse models of cerebellar ataxia. J Vis Exp.

Haas, R.H., Nasirian, F., Nakano, K., Ward, D., Pay, M., Hill, R., and Shults, C.W. (1995). Low platelet mitochondrial complex I and complex II/III activity in early untreated Parkinson's disease. Ann Neurol 37, 714-722.

Haehner, A., Hummel, T., and Reichmann, H. (2011). Olfactory loss in Parkinson's disease. Parkinsons Dis 2011, 450939.

Hajnal, A., Smith, G.P., and Norgren, R. (2004). Oral sucrose stimulation increases accumbens dopamine in the rat. Am J Physiol Regul Integr Comp Physiol 286, R31-37.

Hampton, T.G., Stasko, M.R., Kale, A., Amende, I., and Costa, A.C. (2004). Gait dynamics in trisomic mice: quantitative neurological traits of Down syndrome. Physiol Behav 82, 381-389.

Hanagasi, H.A., Lees, A., Johnson, J.O., Singleton, A., and Emre, M. (2007). Smokingresponsive juvenile-onset Parkinsonism. Mov Disord 22, 115-119.

Herkenham, M., Little, M.D., Bankiewicz, K., Yang, S.C., Markey, S.P., and Johannessen, J.N. (1991). Selective retention of MPP+ within the monoaminergic systems of the primate brain following MPTP administration: an in vivo autoradiographic study. Neuroscience 40, 133-158.

Hershko, A., and Ciechanover, A. (1998). The ubiquitin system. Annu Rev Biochem 67, 425-479.

Hirrlinger, P.G., Scheller, A., Braun, C., Hirrlinger, J., and Kirchhoff, F. (2006). Temporal control of gene recombination in astrocytes by transgenic expression of the tamoxifeninducible DNA recombinase variant CreERT2. Glia 54, 11-20.

Hsu, J.-M., Lee, Y.-C.G., Yu, C.-T.R., and Huang, C.-Y.F. (2004). Fbx7 Functions in the SCF Complex Regulating Cdk1-Cyclin B-phosphorylated Hepatoma Up-regulated Protein (HURP) Proteolysis by a Proline-rich Region. Journal of Biological Chemistry 279, 3259232602.

Huang, Y.L., Chiu, A.W., Huan, S.K., Wang, Y.C., Ju, J.P., and Lu, C.L. (2003). Prognostic significance of hepatoma-up-regulated protein expression in patients with urinary bladder transitional cell carcinoma. Anticancer Res 23, 2729-2733.

li, K., Ito, H., Tanaka, K., and Hirano, A. (1997). Immunocytochemical co-localization of the proteasome in ubiquitinated structures in neurodegenerative diseases and the elderly. $\mathrm{J}$ Neuropathol Exp Neurol 56, 125-131. 
Ikeda, F., and Dikic, I. (2008). Atypical ubiquitin chains: new molecular signals. 'Protein Modifications: Beyond the Usual Suspects' review series. EMBO Rep 9, 536-542.

Ilyin, G.P., Rialland, M., Pigeon, C., and Guguen-Guillouzo, C. (2000). cDNA cloning and expression analysis of new members of the mammalian F-box protein family. Genomics 67, 40-47.

Jamart, C., Raymackers, J.M., Li An, G., Deldicque, L., and Francaux, M. (2011). Prevention of muscle disuse atrophy by MG132 proteasome inhibitor. Muscle Nerve 43, 708-716.v

Jin, J., Cardozo, T., Lovering, R.C., Elledge, S.J., Pagano, M., and Harper, J.W. (2004). Systematic analysis and nomenclature of mammalian F-box proteins. Genes Dev 18, 2573-2580.

Kamadurai, H.B., Qiu, Y., Deng, A., Harrison, J.S., Macdonald, C., Actis, M., Rodrigues, P., Miller, D.J., Souphron, J., Lewis, S.M., et al. (2013). Mechanism of ubiquitin ligation and lysine prioritization by a HECT E3. Elife 2, e00828.

Kale, A., Amende, I., Meyer, G.P., Crabbe, J.C., and Hampton, T.G. (2004). Ethanol's effects on gait dynamics in mice investigated by ventral plane videography. Alcohol Clin Exp Res 28, 1839-1848.

Kang, J., and Chung, K.C. (2015). The F-box protein FBXO7 positively regulates bone morphogenetic protein-mediated signaling through Lys-63-specific ubiquitination of neurotrophin receptor-interacting MAGE (NRAGE). Cell Mol Life Sci 72, 181-195.

Kato, H., Araki, T., Imai, Y., Takahashi, A., and Itoyama, Y. (2003). Protection of dopaminergic neurons with a novel astrocyte modulating agent (R)-(-)-2-propyloctanoic acid (ONO-2506) in an MPTP-mouse model of Parkinson's disease. J Neurol Sci 208, 915.

Keeney, P.M., Xie, J., Capaldi, R.A., and Bennett, J.P., Jr. (2006). Parkinson's disease brain mitochondrial complex I has oxidatively damaged subunits and is functionally impaired and misassembled. J Neurosci 26, 5256-5264.

Kett, L.R., Stiller, B., Bernath, M.M., Tasset, I., Blesa, J., Jackson-Lewis, V., Chan, R.B., Zhou, B., Di Paolo, G., Przedborski, S., et al. (2015). alpha-Synuclein-independent histopathological and motor deficits in mice lacking the endolysosomal Parkinsonism protein Atp13a2. J Neurosci 35, 5724-5742.

Kirk, R., Laman, H., Knowles, P.P., Murray-Rust, J., Lomonosov, M., Meziane el, K., and McDonald, N.Q. (2008). Structure of a conserved dimerization domain within the Fbox protein Fbxo7 and the PI31 proteasome inhibitor. J Biol Chem 283, 22325-22335.

Kisselev, A.F., and Goldberg, A.L. (2005). Monitoring activity and inhibition of $26 \mathrm{~S}$ proteasomes with fluorogenic peptide substrates. Methods Enzymol 398, 364-378.

Kitada, T., Asakawa, S., Hattori, N., Matsumine, H., Yamamura, Y., Minoshima, S., Yokochi, M., Mizuno, Y., and Shimizu, N. (1998). Mutations in the parkin gene cause autosomal recessive juvenile parkinsonism. Nature 392, 605-608.

Kitada, T., Pisani, A., Porter, D.R., Yamaguchi, H., Tscherter, A., Martella, G., Bonsi, P., Zhang, C., Pothos, E.N., and Shen, J. (2007). Impaired dopamine release and synaptic plasticity in the striatum of PINK1-deficient mice. Proc Natl Acad Sci U S A 104, 11441 11446. 
Kitada, T., Tong, Y., Gautier, C.A., and Shen, J. (2009). Absence of nigral degeneration in aged parkin/DJ-1/PINK1 triple knockout mice. J Neurochem 111, 696-702.

Kitajima, Y., Tashiro, Y., Suzuki, N., Warita, H., Kato, M., Tateyama, M., Ando, R., Izumi, R., Yamazaki, M., Abe, M., et al. (2014). Proteasome dysfunction induces muscle growth defects and protein aggregation. J Cell Sci 127, 5204-5217.

Klein, C., and Westenberger, A. (2012). Genetics of Parkinson's disease. Cold Spring Harb Perspect Med 2, a008888.

Konishi, Y., Stegmuller, J., Matsuda, T., Bonni, S., and Bonni, A. (2004). Cdh1-APC controls axonal growth and patterning in the mammalian brain. Science 303, 1026-1030.

Kordower, J.H., Kanaan, N.M., Chu, Y., Suresh Babu, R., Stansell, J., 3rd, Terpstra, B.T., Sortwell, C.E., Steece-Collier, K., and Collier, T.J. (2006). Failure of proteasome inhibitor administration to provide a model of Parkinson's disease in rats and monkeys. Ann Neurol 60, 264-268.

Krebiehl, G., Ruckerbauer, S., Burbulla, L.F., Kieper, N., Maurer, B., Waak, J., Wolburg, H., Gizatullina, Z., Gellerich, F.N., Woitalla, D., et al. (2010). Reduced basal autophagy and impaired mitochondrial dynamics due to loss of Parkinson's disease-associated protein DJ-1. PLoS ONE 5, e9367.

Kuiken, H.J., Egan, D.A., Laman, H., Bernards, R., Beijersbergen, R.L., and Dirac, A.M. (2012). Identification of F-box only protein 7 as a negative regulator of NF-kappaB signaling. Journal of Cellular and Molecular Medicine, n/a-n/a.

LaBaer, J., Garrett, M.D., Stevenson, L.F., Slingerland, J.M., Sandhu, C., Chou, H.S., Fattaey, A., and Harlow, E. (1997). New functional activities for the p21 family of CDK inhibitors. Genes Dev 11, 847-862.

Laman, H., Funes, J.M., Ye, H., Henderson, S., Galinanes-Garcia, L., Hara, E., Knowles, P., McDonald, N., and Boshoff, C. (2005). Transforming activity of Fbxo7 is mediated specifically through regulation of cyclin D/cdk6. EMBO J 24, 3104-3116.

Lee, J.E., Sweredoski, M.J., Graham, R.L., Kolawa, N.J., Smith, G.T., Hess, S., and Deshaies, R.J. (2011). The steady-state repertoire of human SCF ubiquitin ligase complexes does not require ongoing Nedd8 conjugation. Mol Cell Proteomics 10, M110 006460.

Lesage, S., Leutenegger, A.L., and Brice, A. (2005). [LRRK2: a gene belonging to the ROCO family is implicated in the Parkinson's disease]. Med Sci (Paris) 21, 1015-1017.

Li, L.H., Qin, H.Z., Wang, J.L., Wang, J., Wang, X.L., and Gao, G.D. (2009a). Axonal degeneration of nigra-striatum dopaminergic neurons induced by 1-methyl-4-phenyl1,2,3,6-tetrahydropyridine in mice. J Int Med Res 37, 455-463.

Li, X., Thompson, D., Kumar, B., and DeMartino, G.N. (2014). Molecular and cellular roles of PI31 (PSMF1) protein in regulation of proteasome function. J Biol Chem 289, 17392-17405.

Li, Y., Liu, W., Oo, T.F., Wang, L., Tang, Y., Jackson-Lewis, V., Zhou, C., Geghman, K., Bogdanov, M., Przedborski, S., et al. (2009). Mutant LRRK2(R1441G) BAC transgenic mice recapitulate cardinal features of Parkinson's disease. Nat Neurosci 12, 826-828.

Lin, C.H., Chen, M.L., Lai, T.T., Tai, C.H., and Wu, R.M. (2013). Mutational analysis of FBXO7 gene in Parkinson's disease in a Taiwanese population. Neurobiol Aging 34, 1713 e1711-1714. 
Lin, X., Parisiadou, L., Gu, X.L., Wang, L., Shim, H., Sun, L., Xie, C., Long, C.X., Yang, W.J., Ding, J., et al. (2009). Leucine-rich repeat kinase 2 regulates the progression of neuropathology induced by Parkinson's-disease-related mutant alpha-synuclein. Neuron $64,807-827$.

Livnat-Levanon, N., Kevei, E., Kleifeld, O., Krutauz, D., Segref, A., Rinaldi, T., Erpapazoglou, Z., Cohen, M., Reis, N., Hoppe, T., et al. (2014). Reversible 26S proteasome disassembly upon mitochondrial stress. Cell Rep 7, 1371-1380.

Lohmann, E., Coquel, A.S., Honore, A., Gurvit, H., Hanagasi, H., Emre, M., Leutenegger, A.L., Drouet, V., Sahbatou, M., Guven, G., et al. (2015). A new F-box protein 7 gene mutation causing typical Parkinson's disease. Mov Disord 30, 1130-1133.

Lopiano, L., Fasano, M., Giraudo, S., Digilio, G., Koenig, S.H., Torre, E., Bergamasco, B., and Aime, S. (2000). Nuclear magnetic relaxation dispersion profiles of substantia nigra pars compacta in Parkinson's disease patients are consistent with protein aggregation. Neurochem Int 37, 331-336.

Lotharius, J., and Brundin, P. (2002). Impaired dopamine storage resulting from alphasynuclein mutations may contribute to the pathogenesis of Parkinson's disease. Hum Mol Genet 11, 2395-2407.

Lucking, C.B., Durr, A., Bonifati, V., Vaughan, J., De Michele, G., Gasser, T., Harhangi, B.S., Meco, G., Denefle, P., Wood, N.W., et al. (2000). Association between early-onset Parkinson's disease and mutations in the parkin gene. N Engl J Med 342, 1560-1567.

Luk, K.C., Kehm, V., Carroll, J., Zhang, B., O'Brien, P., Trojanowski, J.Q., and Lee, V.M. (2012). Pathological alpha-synuclein transmission initiates Parkinson-like neurodegeneration in nontransgenic mice. Science 338, 949-953.

Lundblad, M., Decressac, M., Mattsson, B., and Bjorklund, A. (2012). Impaired neurotransmission caused by overexpression of alpha-synuclein in nigral dopamine neurons. Proc Natl Acad Sci U S A 109, 3213-3219.

Luong, T.N., Carlisle, H.J., Southwell, A., and Patterson, P.H. (2011). Assessment of motor balance and coordination in mice using the balance beam. J Vis Exp.

Ma, S.Y., Roytta, M., Rinne, J.O., Collan, Y., and Rinne, U.K. (1997). Correlation between neuromorphometry in the substantia nigra and clinical features in Parkinson's disease using disector counts. J Neurol Sci 151, 83-87.

Mahoney, D.J., Cheung, H.H., Mrad, R.L., Plenchette, S., Simard, C., Enwere, E., Arora, V., Mak, T.W., Lacasse, E.C., Waring, J., et al. (2008). Both clAP1 and clAP2 regulate TNFalpha-mediated NF-kappaB activation. Proc Natl Acad Sci U S A 105, 11778-11783.

Manning-Bog, A.B., Reaney, S.H., Chou, V.P., Johnston, L.C., McCormack, A.L., Johnston, J., Langston, J.W., and Di Monte, D.A. (2006). Lack of nigrostriatal pathology in a rat model of proteasome inhibition. Ann Neurol 60, 256-260.

Martin, L.J., Pan, Y., Price, A.C., Sterling, W., Copeland, N.G., Jenkins, N.A., Price, D.L., and Lee, M.K. (2006). Parkinson's disease alpha-synuclein transgenic mice develop neuronal mitochondrial degeneration and cell death. J Neurosci 26, 41-50.

Masliah, E., Rockenstein, E., Veinbergs, I., Mallory, M., Hashimoto, M., Takeda, A., Sagara, Y., Sisk, A., and Mucke, L. (2000). Dopaminergic loss and inclusion body formation in alpha-synuclein mice: implications for neurodegenerative disorders. Science $287,1265-1269$. 
Matluk, N., Rochira, J.A., Karaczyn, A., Adams, T., and Verdi, J.M. (2010). A role for NRAGE in NF-kappaB activation through the non-canonical BMP pathway. BMC Biol 8, 7.

Matsuoka, Y., Vila, M., Lincoln, S., McCormack, A., Picciano, M., LaFrancois, J., Yu, X., Dickson, D., Langston, W.J., McGowan, E., et al. (2001). Lack of nigral pathology in transgenic mice expressing human alpha-synuclein driven by the tyrosine hydroxylase promoter. Neurobiol Dis 8, 535-539.

Matta, S., Van Kolen, K., da Cunha, R., van den Bogaart, G., Mandemakers, W., Miskiewicz, K., De Bock, P.J., Morais, V.A., Vilain, S., Haddad, D., et al. (2012). LRRK2 controls an EndoA phosphorylation cycle in synaptic endocytosis. Neuron 75, 1008-1021.

Mattson, M.P., Goodman, Y., Luo, H., Fu, W., and Furukawa, K. (1997). Activation of NF-kappaB protects hippocampal neurons against oxidative stress-induced apoptosis: evidence for induction of manganese superoxide dismutase and suppression of peroxynitrite production and protein tyrosine nitration. J Neurosci Res 49, 681-697.

McCutchen-Maloney, S.L., Matsuda, K., Shimbara, N., Binns, D.D., Tanaka, K., Slaughter, C.A., and DeMartino, G.N. (2000). cDNA cloning, expression, and functional characterization of PI31, a proline-rich inhibitor of the proteasome. J Biol Chem 275, 18557-18565.

McNaught, K.S., Belizaire, R., Jenner, P., Olanow, C.W., and Isacson, O. (2002a). Selective loss of $20 \mathrm{~S}$ proteasome alpha-subunits in the substantia nigra pars compacta in Parkinson's disease. Neurosci Lett 326, 155-158.

McNaught, K.S., Bjorklund, L.M., Belizaire, R., Isacson, O., Jenner, P., and Olanow, C.W. (2002b). Proteasome inhibition causes nigral degeneration with inclusion bodies in rats. Neuroreport 13, 1437-1441.

McNaught, K.S., and Jenner, P. (2001). Proteasomal function is impaired in substantia nigra in Parkinson's disease. Neurosci Lett 297, 191-194.

McNaught, K.S., Perl, D.P., Brownell, A.L., and Olanow, C.W. (2004). Systemic exposure to proteasome inhibitors causes a progressive model of Parkinson's disease. Ann Neurol 56, 149-162.

McNaught, K.S., Olanow, C.W., Halliwell, B., Isacson, O., and Jenner, P. (2001). Failure of the ubiquitin-proteasome system in Parkinson's disease. Nat Rev Neurosci 2, 589-594.

Melrose, H.L., Dachsel, J.C., Behrouz, B., Lincoln, S.J., Yue, M., Hinkle, K.M., Kent, C.B., Korvatska, E., Taylor, J.P., Witten, L., et al. (2010). Impaired dopaminergic neurotransmission and microtubule-associated protein tau alterations in human LRRK2 transgenic mice. Neurobiol Dis 40, 503-517.

Meziane el, K., Randle, S.J., Nelson, D.E., Lomonosov, M., and Laman, H. (2011). Knockdown of $\mathrm{Fbxo7}$ reveals its regulatory role in proliferation and differentiation of haematopoietic precursor cells. J Cell Sci 124, 2175-2186.

Morgan, D.O. (1995). Principles of CDK regulation. Nature 374, 131-134.

Nelson, D.E., Randle, S.J., and Laman, H. (2013). Beyond ubiquitination: the atypical functions of Fbxo7 and other F-box proteins. Open Biol 3, 130131.

Nelson, D.E., and Laman, H. (2011). A Competitive binding mechanism between Skp1 and exportin 1 (CRM1) controls the localization of a subset of F-box proteins. J Biol Chem 286, 19804-19815. 
Nemani, V.M., Lu, W., Berge, V., Nakamura, K., Onoa, B., Lee, M.K., Chaudhry, F.A., Nicoll, R.A., and Edwards, R.H. (2010). Increased expression of alpha-synuclein reduces neurotransmitter release by inhibiting synaptic vesicle reclustering after endocytosis. Neuron 65, 66-79.

Nuytemans, K., Theuns, J., Cruts, M., and Van Broeckhoven, C. (2010). Genetic etiology of Parkinson disease associated with mutations in the SNCA, PARK2, PINK1, PARK7, and LRRK2 genes: a mutation update. Hum Mutat 31, 763-780.

Ogawa, N., Hirose, Y., Ohara, S., Ono, T., and Watanabe, Y. (1985). A simple quantitative bradykinesia test in MPTP-treated mice. Res Commun Chem Pathol Pharmacol 50, 435-441.

Olanow, C.W., and Brundin, P. (2013). Parkinson's disease and alpha synuclein: is Parkinson's disease a prion-like disorder? Mov Disord 28, 31-40.

Orlicky, S., Tang, X., Willems, A., Tyers, M., and Sicheri, F. (2003). Structural basis for phosphodependent substrate selection and orientation by the SCFCdc4 ubiquitin ligase. Cell 112, 243-256.

Ozelius, L.J., Senthil, G., Saunders-Pullman, R., Ohmann, E., Deligtisch, A., Tagliati, M., Hunt, A.L., Klein, C., Henick, B., Hailpern, S.M., et al. (2006). LRRK2 G2019S as a cause of Parkinson's disease in Ashkenazi Jews. N Engl J Med 354, 424-425.

Paisan-Ruiz, C., Guevara, R., Federoff, M., Hanagasi, H., Sina, F., Elahi, E., Schneider, S.A., Schwingenschuh, P., Bajaj, N., Emre, M., et al. (2010). Early-onset L-doparesponsive parkinsonism with pyramidal signs due to ATP13A2, PLA2G6, FBXO7 and spatacsin mutations. Mov Disord 25, 1791-1800.

Papworth C., Bauer J.C., Braman J., Wright D.A. (1996) Site-directed mutagenesis in 1 day with $>80 \%$ efficiency. Strategies $9,3-4$.

Parker, W.D., Jr., Boyson, S.J., and Parks, J.K. (1989). Abnormalities of the electron transport chain in idiopathic Parkinson's disease. Ann Neurol 26, 719-723.

Perez, F.A., and Palmiter, R.D. (2005). Parkin-deficient mice are not a robust model of parkinsonism. Proc Natl Acad Sci U S A 102, 2174-2179.

Piccoli, G., Condliffe, S.B., Bauer, M., Giesert, F., Boldt, K., De Astis, S., Meixner, A., Sarioglu, H., Vogt-Weisenhorn, D.M., Wurst, W., et al. (2011). LRRK2 controls synaptic vesicle storage and mobilization within the recycling pool. J Neurosci 31, 2225-2237.

Pickart, C.M. (2000). Ubiquitin in chains. Trends Biochem Sci 25, 544-548.

Pickart, C.M. (2004). Back to the future with ubiquitin. Cell 116, 181-190.

Pickart, C.M., and Eddins, M.J. (2004). Ubiquitin: structures, functions, mechanisms. Biochim Biophys Acta 1695, 55-72.

Polymeropoulos, M.H., Lavedan, C., Leroy, E., Ide, S.E., Dehejia, A., Dutra, A., Pike, B., Root, H., Rubenstein, J., Boyer, R., et al. (1997). Mutation in the alpha-synuclein gene identified in families with Parkinson's disease. Science 276, 2045-2047.

Poulopoulos, M., Levy, O.A., and Alcalay, R.N. (2012). The neuropathology of genetic Parkinson's disease. Mov Disord 27, 831-842.

Przedborski, S. (2007). Neuroinflammation and Parkinson's disease. Handb Clin Neurol 83, 535-551. 
Rada, P., Bocarsly, M.E., Barson, J.R., Hoebel, B.G., and Leibowitz, S.F. (2010). Reduced accumbens dopamine in Sprague-Dawley rats prone to overeating a fat-rich diet. Physiol Behav 101, 394-400.

Ramonet, D., Daher, J.P., Lin, B.M., Stafa, K., Kim, J., Banerjee, R., Westerlund, M., Pletnikova, O., Glauser, L., Yang, L., et al. (2011). Dopaminergic neuronal loss, reduced neurite complexity and autophagic abnormalities in transgenic mice expressing G2019S mutant LRRK2. PLoS ONE 6, e18568.

Randle, S.J., Nelson, D.E., Patel, S.P., and Laman, H. (2015). Defective erythropoiesis in a mouse model of reduced Fbxo7 expression due to decreased p27 expression. $J$ Pathol.

Rizzuto, R., Duchen, M.R., and Pozzan, T. (2004). Flirting in little space: the ER/mitochondria Ca2+ liaison. Sci STKE 2004, re1.

Rockenstein, E., Mallory, M., Hashimoto, M., Song, D., Shults, C.W., Lang, I., and Masliah, E. (2002). Differential neuropathological alterations in transgenic mice expressing alpha-synuclein from the platelet-derived growth factor and Thy-1 promoters. $J$ Neurosci Res 68, 568-578.

Romanello, V., Guadagnin, E., Gomes, L., Roder, I., Sandri, C., Petersen, Y., Milan, G., Masiero, E., Del Piccolo, P., Foretz, M., et al. (2010). Mitochondrial fission and remodelling contributes to muscle atrophy. EMBO J 29, 1774-1785.

Rommelfanger, K.S., Edwards, G.L., Freeman, K.G., Liles, L.C., Miller, G.W., and Weinshenker, D. (2007). Norepinephrine loss produces more profound motor deficits than MPTP treatment in mice. Proc Natl Acad Sci U S A 104, 13804-13809.

Rousseaux, M.W., Marcogliese, P.C., Qu, D., Hewitt, S.J., Seang, S., Kim, R.H., Slack, R.S., Schlossmacher, M.G., Lagace, D.C., Mak, T.W., et al. (2012). Progressive dopaminergic cell loss with unilateral-to-bilateral progression in a genetic model of Parkinson disease. Proc Natl Acad Sci U S A 109, 15918-15923.

Saigoh, K., Wang, Y.L., Suh, J.G., Yamanishi, T., Sakai, Y., Kiyosawa, H., Harada, T., Ichihara, N., Wakana, S., Kikuchi, T., et al. (1999). Intragenic deletion in the gene encoding ubiquitin carboxy-terminal hydrolase in gad mice. Nat Genet 23, 47-51.

Saiki, R.K., Scharf, S., Faloona, F., Mullis, K.B., Horn, G.T., Erlich, H.A., and Arnheim, N. (1985). Enzymatic amplification of beta-globin genomic sequences and restriction site analysis for diagnosis of sickle cell anemia. Science 230, 1350-1354.

Saura, J., Pares, M., Bove, J., Pezzi, S., Alberch, J., Marin, C., Tolosa, E., and Marti, M.J. (2003). Intranigral infusion of interleukin-1beta activates astrocytes and protects from subsequent 6-hydroxydopamine neurotoxicity. J Neurochem 85, 651-661.

Savitt, J.M., Jang, S.S., Mu, W., Dawson, V.L., and Dawson, T.M. (2005). Bcl-x is required for proper development of the mouse substantia nigra. J Neurosci 25, 67216728.

Schapira, A.H., Cooper, J.M., Dexter, D., Jenner, P., Clark, J.B., and Marsden, C.D. (1989). Mitochondrial complex I deficiency in Parkinson's disease. Lancet 1, 1269.

Scheffner, M., Nuber, U., and Huibregtse, J.M. (1995). Protein ubiquitination involving an E1-E2-E3 enzyme ubiquitin thioester cascade. Nature 373, 81-83. 
Schmeisser, M.J., Ey, E., Wegener, S., Bockmann, J., Stempel, A.V., Kuebler, A., Janssen, A.L., Udvardi, P.T., Shiban, E., Spilker, C., et al. (2012). Autistic-like behaviours and hyperactivity in mice lacking ProSAP1/Shank2. Nature 486, 256-260.

Sedelis, M., Schwarting, R.K., and Huston, J.P. (2001). Behavioral phenotyping of the MPTP mouse model of Parkinson's disease. Behav Brain Res 125, 109-125.

Serra, P.A., Sciola, L., Delogu, M.R., Spano, A., Monaco, G., Miele, E., Rocchitta, G., Miele, M., Migheli, R., and Desole, M.S. (2002). The neurotoxin 1-methyl-4-phenyl1,2,3,6-tetrahydropyridine induces apoptosis in mouse nigrostriatal glia. Relevance to nigral neuronal death and striatal neurochemical changes. J Biol Chem 277, 3445134461.

Shang, J., Huang, X., and Du, Z. (2015). The FP domains of PI31 and Fbxo7 have the same protein fold but very different modes of protein-protein interaction. J Biomol Struct Dyn 33, 1528-1538.

Shang, J., Wang, G., Yang, Y., Huang, X., and Du, Z. (2014). Structure of the FP domain of Fbxo7 reveals a novel mode of protein-protein interaction. Acta Crystallogr $D$ Biol Crystallogr 70, 155-164.

Sherr, C.J. (1996). Cancer cell cycles. Science 274, 1672-1677.

Shojaee, S., Sina, F., Banihosseini, S.S., Kazemi, M.H., Kalhor, R., Shahidi, G.A., Fakhrai-Rad, H., Ronaghi, M., and Elahi, E. (2008). Genome-wide linkage analysis of a Parkinsonian-pyramidal syndrome pedigree by $500 \mathrm{~K}$ SNP arrays. Am J Hum Genet 82, 1375-1384.

Sonsalla, P.K., and Heikkila, R.E. (1988). Neurotoxic effects of 1-methyl-4-phenyl1,2,3,6-tetrahydropyridine (MPTP) and methamphetamine in several strains of mice. Prog Neuropsychopharmacol Biol Psychiatry 12, 345-354.

Soranzo, N., Spector, T.D., Mangino, M., Kuhnel, B., Rendon, A., Teumer, A., Willenborg, C., Wright, B., Chen, L., Li, M., et al. (2009). A genome-wide meta-analysis identifies 22 loci associated with eight hematological parameters in the HaemGen consortium. Nat Genet 41, 1182-1190.

Sortwell, C.E., Daley, B.F., Pitzer, M.R., McGuire, S.O., Sladek, J.R., Jr., and Collier, T.J. (2000). Oligodendrocyte-type 2 astrocyte-derived trophic factors increase survival of developing dopamine neurons through the inhibition of apoptotic cell death. J Comp Neurol 426, 143-153.

Sotak, B.N., Hnasko, T.S., Robinson, S., Kremer, E.J., and Palmiter, R.D. (2005). Dysregulation of dopamine signaling in the dorsal striatum inhibits feeding. Brain Res 1061, 88-96.

Spillantini, M.G., Schmidt, M.L., Lee, V.M., Trojanowski, J.Q., Jakes, R., and Goedert, M. (1997). Alpha-synuclein in Lewy bodies. Nature 388, 839-840.

Stegmuller, J., Konishi, Y., Huynh, M.A., Yuan, Z., Dibacco, S., and Bonni, A. (2006). Cell-intrinsic regulation of axonal morphogenesis by the Cdh1-APC target SnoN. Neuron $50,389-400$.

Stein, T.D., and Johnson, J.A. (2002). Lack of neurodegeneration in transgenic mice overexpressing mutant amyloid precursor protein is associated with increased levels of transthyretin and the activation of cell survival pathways. J Neurosci 22, 7380-7388. 
Strekalova, T., and Steinbusch, H.W. (2010). Measuring behavior in mice with chronic stress depression paradigm. Prog Neuropsychopharmacol Biol Psychiatry 34, 348-361.

Sullivan, P.G., Dragicevic, N.B., Deng, J.H., Bai, Y., Dimayuga, E., Ding, Q., Chen, Q., Bruce-Keller, A.J., and Keller, J.N. (2004). Proteasome inhibition alters neural mitochondrial homeostasis and mitochondria turnover. J Biol Chem 279, 20699-20707.

Swonger, A.K., and Rech, R.H. (1972). Serotonergic and cholinergic involvement in habituation of activity and spontaneous alternation of rats in a $\mathrm{Y}$ maze. J Comp Physiol Psychol 81, 509-522.

Taira, T., Saito, Y., Niki, T., Iguchi-Ariga, S.M., Takahashi, K., and Ariga, H. (2004). DJ1 has a role in antioxidative stress to prevent cell death. EMBO Rep 5, 213-218.

Takei, Y., Teng, J., Harada, A., and Hirokawa, N. (2000). Defects in axonal elongation and neuronal migration in mice with disrupted tau and map1b genes. J Cell Biol 150, 9891000.

Tan, M., Davis, S.W., Saunders, T.L., Zhu, Y., and Sun, Y. (2009). RBX1/ROC1 disruption results in early embryonic lethality due to proliferation failure, partially rescued by simultaneous loss of p27. Proc Natl Acad Sci U S A 106, 6203-6208.

Tang, X., Orlicky, S., Lin, Z., Willems, A., Neculai, D., Ceccarelli, D., Mercurio, F., Shilton, B.H., Sicheri, F., and Tyers, M. (2007). Suprafacial orientation of the SCFCdc4 dimer accommodates multiple geometries for substrate ubiquitination. Cell 129, 11651176.

Tashiro, Y., Urushitani, M., Inoue, H., Koike, M., Uchiyama, Y., Komatsu, M., Tanaka, K., Yamazaki, M., Abe, M., Misawa, H., et al. (2012). Motor neuron-specific disruption of proteasomes, but not autophagy, replicates amyotrophic lateral sclerosis. J Biol Chem 287, 42984-42994.

Thomas, B., and Beal, M.F. (2007). Parkinson's disease. Hum Mol Genet 16 Spec No. 2, R183-194.

Tillerson, J.L., and Miller, G.W. (2003). Grid performance test to measure behavioral impairment in the MPTP-treated-mouse model of parkinsonism. J Neurosci Methods 123, 189-200.

Tomko, R.J., Jr., and Hochstrasser, M. (2013). Molecular architecture and assembly of the eukaryotic proteasome. Annu Rev Biochem 82, 415-445.

Tonges, L., Frank, T., Tatenhorst, L., Saal, K.A., Koch, J.C., Szego, E.M., Bahr, M., Weishaupt, J.H., and Lingor, P. (2012). Inhibition of rho kinase enhances survival of dopaminergic neurons and attenuates axonal loss in a mouse model of Parkinson's disease. Brain 135, 3355-3370.

Trevor, A.J., Castagnoli, N., and Singer, T.P. (1988). The formation of reactive intermediates in the MAO-catalyzed oxidation of the nigrostriatal toxin 1-methyl-4-phenyl1,2,3,6-tetrahydropyridine (MPTP). Toxicology 49, 513-519.

Tsou, A.P., Yang, C.W., Huang, C.Y., Yu, R.C., Lee, Y.C., Chang, C.W., Chen, B.R., Chung, Y.F., Fann, M.J., Chi, C.W., et al. (2003). Identification of a novel cell cycle regulated gene, HURP, overexpressed in human hepatocellular carcinoma. Oncogene 22, 298-307. 
Um, J.W., Im, E., Lee, H.J., Min, B., Yoo, L., Yoo, J., Lubbert, H., Stichel-Gunkel, C., Cho, H.S., Yoon, J.B., et al. (2010). Parkin directly modulates $26 S$ proteasome activity. J Neurosci 30, 11805-11814.

Valadas, J.S., Vos, M., and Verstreken, P. (2015). Therapeutic strategies in Parkinson's disease: what we have learned from animal models. Ann N Y Acad Sci 1338, 16-37.

Valente, E.M., Abou-Sleiman, P.M., Caputo, V., Muqit, M.M., Harvey, K., Gispert, S., Ali, Z., Del Turco, D., Bentivoglio, A.R., Healy, D.G., et al. (2004). Hereditary early-onset Parkinson's disease caused by mutations in PINK1. Science 304, 1158-1160.

van der Harst, P., Zhang, W., Mateo Leach, I., Rendon, A., Verweij, N., Sehmi, J., Paul, D.S., Elling, U., Allayee, H., Li, X., et al. (2012). Seventy-five genetic loci influencing the human red blood cell. Nature 492, 369-375.

van Putten, M., de Winter, C., van Roon-Mom, W., van Ommen, G.J., t Hoen, P.A., and Aartsma-Rus, A. (2010). A 3 months mild functional test regime does not affect disease parameters in young mdx mice. Neuromuscul Disord 20, 273-280.

Verstreken, P., Ly, C.V., Venken, K.J., Koh, T.W., Zhou, Y., and Bellen, H.J. (2005). Synaptic mitochondria are critical for mobilization of reserve pool vesicles at Drosophila neuromuscular junctions. Neuron 47, 365-378.

Von Coelln, R., Thomas, B., Savitt, J.M., Lim, K.L., Sasaki, M., Hess, E.J., Dawson, V.L., and Dawson, T.M. (2004). Loss of locus coeruleus neurons and reduced startle in parkin null mice. Proc Natl Acad Sci U S A 101, 10744-10749.

Willeumier, K., Pulst, S.M., and Schweizer, F.E. (2006). Proteasome inhibition triggers activity-dependent increase in the size of the recycling vesicle pool in cultured hippocampal neurons. J Neurosci 26, 11333-11341.

Winston, J.T., Koepp, D.M., Zhu, C., Elledge, S.J., and Harper, J.W. (1999). A family of mammalian F-box proteins. Curr Biol 9, 1180-1182.

Wu, D.C., Teismann, P., Tieu, K., Vila, M., Jackson-Lewis, V., Ischiropoulos, H., and Przedborski, S. (2003). NADPH oxidase mediates oxidative stress in the 1-methyl-4phenyl-1,2,3,6-tetrahydropyridine model of Parkinson's disease. Proc Natl Acad Sci U S A $100,6145-6150$.

Xiong, N., Long, X., Xiong, J., Jia, M., Chen, C., Huang, J., Ghoorah, D., Kong, X., Lin, Z., and Wang, T. (2012). Mitochondrial complex I inhibitor rotenone-induced toxicity and its potential mechanisms in Parkinson's disease models. Crit Rev Toxicol 42, 613-632.

Yalcin-Cakmakli, G., Olgiati, S., Quadri, M., Breedveld, G.J., Cortelli, P., Bonifati, V., and Elibol, B. (2014). A new Turkish family with homozygous FBXO7 truncating mutation and juvenile atypical parkinsonism. Parkinsonism Relat Disord 20, 1248-1252.

Yokota, T., Sugawara, K., Ito, K., Takahashi, R., Ariga, H., and Mizusawa, H. (2003). Down regulation of $\mathrm{DJ}-1$ enhances cell death by oxidative stress, ER stress, and proteasome inhibition. Biochem Biophys Res Commun 312, 1342-1348.

Yonashiro, R., Kimijima, Y., Shimura, T., Kawaguchi, K., Fukuda, T., Inatome, R., and Yanagi, S. (2012). Mitochondrial ubiquitin ligase MITOL blocks S-nitrosylated MAP1B-light chain 1-mediated mitochondrial dysfunction and neuronal cell death. Proc Natl Acad Sci U S A 109, 2382-2387. 
Yun, H.J., Park, J., Ho, D.H., Kim, H., Kim, C.H., Oh, H., Ga, I., Seo, H., Chang, S., Son, I., et al. (2013). LRRK2 phosphorylates Snapin and inhibits interaction of Snapin with SNAP-25. Exp Mol Med 45, e36.

Zaiss, D.M., Standera, S., Holzhutter, H., Kloetzel, P., and Sijts, A.J. (1999). The proteasome inhibitor PI31 competes with PA28 for binding to $20 \mathrm{~S}$ proteasomes. FEBS Lett 457, 333-338.

Zaiss, D.M., Standera, S., Kloetzel, P.M., and Sijts, A.J. (2002). PI31 is a modulator of proteasome formation and antigen processing. Proc Natl Acad Sci U S A 99, 1434414349.

Zarow, C., Lyness, S.A., Mortimer, J.A., and Chui, H.C. (2003). Neuronal loss is greater in the locus coeruleus than nucleus basalis and substantia nigra in Alzheimer and Parkinson diseases. Arch Neurol 60, 337-341.

Zhang, H., and Sulzer, D. (2003). Glutamate spillover in the striatum depresses dopaminergic transmission by activating group I metabotropic glutamate receptors. J Neurosci 23, 10585-10592.

Zhang, Y., Chen, K., Sloan, S.A., Bennett, M.L., Scholze, A.R., O'Keeffe, S., Phatnani, H.P., Guarnieri, P., Caneda, C., Ruderisch, N., et al. (2014). An RNA-sequencing transcriptome and splicing database of glia, neurons, and vascular cells of the cerebral cortex. J Neurosci 34, 11929-11947.

Zhao, T., De Graaff, E., Breedveld, G.J., Loda, A., Severijnen, L.A., Wouters, C.H., Verheijen, F.W., Dekker, M.C., Montagna, P., Willemsen, R., et al. (2011). Loss of nuclear activity of the $\mathrm{FBXO7}$ protein in patients with parkinsonian-pyramidal syndrome (PARK15). PLoS ONE 6, e16983.

Zhao, T., Severijnen, L.A., van der Weiden, M., Zheng, P.P., Oostra, B.A., Hukema, R.K., Willemsen, R., Kros, J.M., and Bonifati, V. (2013). FBXO7 immunoreactivity in alpha-synuclein-containing inclusions in Parkinson disease and multiple system atrophy. $\mathrm{J}$ Neuropathol Exp Neurol 72, 482-488.

Zhou, W., Wei, W., and Sun, Y. (2013). Genetically engineered mouse models for functional studies of SKP1-CUL1-F-box-protein (SCF) E3 ubiquitin ligases. Cell Res 23, 599-619.

Zhou, Z.D., Xie, S.P., Sathiyamoorthy, S., Saw, W.T., Sing, T.Y., Ng, S.H., Chua, H.P., Tang, A.M., Shaffra, F., Li, Z., et al. (2015). F-box protein 7 mutations promote protein aggregation in mitochondria and inhibit mitophagy. Hum Mol Genet 24, 6314-6330.

Ziviani, E., Tao, R.N., and Whitworth, A.J. (2010). Drosophila parkin requires PINK1 for mitochondrial translocation and ubiquitinates mitofusin. Proc Natl Acad Sci U S A 107, 5018-5023. 


\section{Appendix}

Table A1 Mutations and clinical features described in PARK15 patients. Modified from YalcinCakmakli et. al (Yalcin-Cakmakli et al., 2014).

\begin{tabular}{|c|c|c|c|c|c|c|}
\hline $\begin{array}{l}\text { Subject ID } \\
\text { code, } \\
\text { gender }\end{array}$ & $\begin{array}{l}\text { ANK-06, } \\
\text { female }\end{array}$ & ANK-07, male & $\begin{array}{l}\text { Family C, } \\
\text { female }\end{array}$ & $\begin{array}{l}\text { Family C, } \\
\text { female }\end{array}$ & $\begin{array}{l}\text { Family C, } \\
\text { female }\end{array}$ & $\begin{array}{l}\text { Family D, } \\
\text { male }\end{array}$ \\
\hline Reference & $\begin{array}{l}\text { (Yalcin- } \\
\text { Cakmakli et } \\
\text { al., 2014) }\end{array}$ & $\begin{array}{l}\text { (Yalcin- } \\
\text { Cakmakli et al., } \\
\text { 2014) }\end{array}$ & $\begin{array}{l}\text { (Paisan-Ruiz } \\
\text { et al., 2010) }\end{array}$ & $\begin{array}{l}\text { (Paisan-Ruiz } \\
\text { et al., 2010) }\end{array}$ & $\begin{array}{l}\text { (Paisan-Ruiz } \\
\text { et al., 2010) }\end{array}$ & $\begin{array}{l}\text { (Paisan-Ruiz } \\
\text { et al., 2010) }\end{array}$ \\
\hline Country & Turkey & Turkey & Pakistan & Pakistan & Pakistan & Turkey \\
\hline Mutations & $\begin{array}{l}\text { Homozygous } \\
\text { p.Arg498* }\end{array}$ & $\begin{array}{l}\text { Homozygous } \\
\text { p.Arg498* }\end{array}$ & $\begin{array}{l}\text { Homozygous } \\
\text { p.Arg498* }\end{array}$ & $\begin{array}{l}\text { Homozygous } \\
\text { p.Arg498* }\end{array}$ & $\begin{array}{l}\text { Homozygous } \\
\text { p.Arg498* }\end{array}$ & $\begin{array}{l}\text { Homozygous } \\
\text { p.Arg498* }\end{array}$ \\
\hline $\begin{array}{l}\text { Mental } \\
\text { retardation }\end{array}$ & + & + & na & na & na & - \\
\hline Onset age & 10 & 14 & 17 & 24 & 22 & 17 \\
\hline $\begin{array}{l}\text { Symptoms } \\
\text { at onset }\end{array}$ & $\begin{array}{l}\text { Slowness, } \\
\text { gait } \\
\text { imbalance, } \\
\text { falls }\end{array}$ & $\begin{array}{l}\text { Slowness, gait } \\
\text { imbalance, falls }\end{array}$ & $\begin{array}{l}\text { Eye-opening } \\
\text { difficulties, } \\
\text { stiffness }\end{array}$ & Slowness & Slowness & $\begin{array}{l}\text { Walking } \\
\text { difficulties, } \\
\text { stiffness }\end{array}$ \\
\hline $\begin{array}{l}\text { Parkinson } \\
\text { signs }\end{array}$ & $\begin{array}{l}\text { Bradykinesia, } \\
\text { rigidity, } \\
\text { postural } \\
\text { instability }\end{array}$ & $\begin{array}{l}\text { Bradykinesia, } \\
\text { rigidity, postural } \\
\text { instability, } \\
\text { resting tremor }\end{array}$ & $\begin{array}{l}\text { Bradykinesia, } \\
\text { rigidity, } \\
\text { postural } \\
\text { instability }\end{array}$ & $\begin{array}{l}\text { Bradykinesia, } \\
\text { rigidity, } \\
\text { postural } \\
\text { instability }\end{array}$ & $\begin{array}{l}\text { Bradykinesia, } \\
\text { rigidity, } \\
\text { postural } \\
\text { instability }\end{array}$ & $\begin{array}{l}\text { Bradykinesia, } \\
\text { rigidity, } \\
\text { postural } \\
\text { instability }\end{array}$ \\
\hline $\begin{array}{l}\text { Pyramidal } \\
\text { signs }\end{array}$ & - & - & + & + & + & - \\
\hline $\begin{array}{l}\text { Additional } \\
\text { symptoms }\end{array}$ & & $\begin{array}{l}\text { Dysphagia, } \\
\text { tongue } \\
\text { protrusion }\end{array}$ & $\begin{array}{l}\text { Cataracts, } \\
\text { eye-opening } \\
\text { apraxia }\end{array}$ & $\begin{array}{l}\text { Dysphagia, } \\
\text { cataracts }\end{array}$ & $\begin{array}{l}\text { Dysphagia, } \\
\text { dystonia }\end{array}$ & $\begin{array}{l}\text { Marked } \\
\text { response to } \\
\text { nicotine }\end{array}$ \\
\hline $\begin{array}{l}\text { Response } \\
\text { to L-Dopa }\end{array}$ & + & + & + & + & + & + \\
\hline $\begin{array}{l}\text { Side- } \\
\text { effetcs of } \\
\text { L-Dopa }\end{array}$ & $\begin{array}{l}\text { Dyskinesias, } \\
\text { behavioral } \\
\text { disturbances }\end{array}$ & $\begin{array}{l}\text { Dyskinesias, } \\
\text { behavioral } \\
\text { disturbances }\end{array}$ & $\begin{array}{l}\text { Dyskinesias, } \\
\text { behavioral } \\
\text { disturbances }\end{array}$ & Dyskinesias & Dyskinesias & $\begin{array}{l}\text { Dyskinesias, } \\
\text { psychosis }\end{array}$ \\
\hline Brain MRI & Normal & $\begin{array}{l}\text { Unspecific T2 } \\
\text { hyperintensities }\end{array}$ & Normal & $\begin{array}{l}\text { General } \\
\text { atrophy }\end{array}$ & $\begin{array}{l}\text { General } \\
\text { atrophy }\end{array}$ & Normal \\
\hline
\end{tabular}




\begin{tabular}{|c|c|c|c|c|c|c|}
\hline $\begin{array}{l}\text { Subject ID } \\
\text { code, } \\
\text { gender }\end{array}$ & $\begin{array}{l}\text { NIJ-002, } \\
\text { female }\end{array}$ & $\begin{array}{l}\text { NIJ-006, } \\
\text { male }\end{array}$ & $\begin{array}{l}10 \text { patients, } \\
8 \text { males, } 2 \\
\text { females }\end{array}$ & Y119, female & Y186, female & $\begin{array}{l}\text { Individual III:2, } \\
\text { female }\end{array}$ \\
\hline Reference & $\begin{array}{l}\text { (Di Fonzo et } \\
\text { al., 2009) }\end{array}$ & $\begin{array}{l}\text { (Di Fonzo et } \\
\text { al., 2009) }\end{array}$ & $\begin{array}{l}\text { (Shojaee et } \\
\text { al., 2008) }\end{array}$ & $\begin{array}{l}\text { (Lin et al., } \\
2013)\end{array}$ & $\begin{array}{l}\text { (Lin et al., } \\
2013 \text { ) }\end{array}$ & $\begin{array}{l}\text { (Lohmann et al., } \\
\text { 2015) }\end{array}$ \\
\hline Country & Netherlands & Netherlands & Iran & Taiwan & Taiwan & Turkey \\
\hline Mutations & $\begin{array}{l}\text { Comp. } \\
\text { heterozygous } \\
\text { c1144+1G>T } \\
\text { pThr22Met }\end{array}$ & $\begin{array}{l}\text { Comp. } \\
\text { heterozygous } \\
\text { c1144+1G>T } \\
\text { pThr22Met }\end{array}$ & $\begin{array}{l}\text { Homozygous } \\
\text { p.Arg378Gly }\end{array}$ & $\begin{array}{l}\text { Heterozygous } \\
\text { p.lle87Thr }\end{array}$ & $\begin{array}{l}\text { Heterozygous } \\
\text { p.Asp328Arg }\end{array}$ & $\begin{array}{l}\text { Homozygous } \\
\text { p.Leu34Arg }\end{array}$ \\
\hline $\begin{array}{l}\text { Mental } \\
\text { retardation }\end{array}$ & - & - & - & - & - & - \\
\hline Onset age & 18 & 19 & Third decade & 42 & 40 & 52 \\
\hline $\begin{array}{l}\text { Symptoms } \\
\text { at onset }\end{array}$ & $\begin{array}{l}\text { Tremor, } \\
\text { nervousness }\end{array}$ & $\begin{array}{l}\text { Social } \\
\text { withdrawal, } \\
\text { slowness }\end{array}$ & Stiffness & na & na & Tremors, cramps \\
\hline $\begin{array}{l}\text { Parkinson } \\
\text { signs }\end{array}$ & $\begin{array}{l}\text { Bradykinesia, } \\
\text { rigidity, } \\
\text { postural } \\
\text { instability }\end{array}$ & $\begin{array}{l}\text { Bradykinesia, } \\
\text { rigidity, } \\
\text { postural } \\
\text { instability, } \\
\text { resting tremor }\end{array}$ & $\begin{array}{l}\text { Bradykinesia, } \\
\text { rigidity (only } \\
3 \text { worst } \\
\text { patients) }\end{array}$ & $\begin{array}{l}\text { Bradykinesia, } \\
\text { rigidity }\end{array}$ & $\begin{array}{l}\text { Bradykinesia, } \\
\text { rigidity }\end{array}$ & $\begin{array}{l}\text { Bradykinesia, } \\
\text { rigidity, resting } \\
\text { tremor }\end{array}$ \\
\hline $\begin{array}{l}\text { Pyramidal } \\
\text { signs }\end{array}$ & + & + & $\begin{array}{l}++(\text { all } \\
\text { patients) }\end{array}$ & na & na & - \\
\hline $\begin{array}{l}\text { Additional } \\
\text { symptoms }\end{array}$ & $\begin{array}{l}\text { Slow } \\
\text { saccades, } \\
\text { upgaze } \\
\text { limitation }\end{array}$ & $\begin{array}{l}\text { Dysphagia, } \\
\text { upgaze } \\
\text { limitation }\end{array}$ & $\begin{array}{l}\text { Equinovarus } \\
\text { deformity (all } \\
\text { patients) }\end{array}$ & na & na & $\begin{array}{l}\text { Dysarthric } \\
\text { speech, } \\
\text { depression }\end{array}$ \\
\hline $\begin{array}{l}\text { Response } \\
\text { to L-Dopa }\end{array}$ & + & + & $\begin{array}{l}+ \text { (only one } \\
\text { patient } \\
\text { treated) }\end{array}$ & + & + & + \\
\hline $\begin{array}{l}\text { Side- } \\
\text { effetcs of } \\
\text { L-Dopa }\end{array}$ & $\begin{array}{l}\text { Dyskinesias, } \\
\text { behavioral } \\
\text { disturbances }\end{array}$ & $\begin{array}{l}\text { Dyskinesias, } \\
\text { behavioral } \\
\text { disturbances }\end{array}$ & na & Dyskinesias & - & Hallucinations \\
\hline Brain MRI & Unremarkable & Unremarkable & Normal & na & na & $\begin{array}{l}\text { Mild cortical } \\
\text { atrophy and } \\
\text { periventritcular } \\
\text { white amtter } \\
\text { lesions }\end{array}$ \\
\hline
\end{tabular}




\begin{tabular}{|c|c|c|c|c|}
\hline $\begin{array}{l}\text { Subject ID code, } \\
\text { gender }\end{array}$ & $\begin{array}{l}\text { Individual III:4, } \\
\text { male }\end{array}$ & BO-53, female & BO-56, male & Patient 1, female \\
\hline Reference & $\begin{array}{l}\text { (Lohmann et al., } \\
\text { 2015) }\end{array}$ & $\begin{array}{l}\text { (Di Fonzo et al., } \\
2009)\end{array}$ & $\begin{array}{l}\text { (Di Fonzo et al., } \\
2009)\end{array}$ & $\begin{array}{l}\text { (Conedera et al., } \\
\text { 2016) }\end{array}$ \\
\hline Country & Turkey & Italy & Italy & Turkey \\
\hline Mutations & $\begin{array}{l}\text { Homozygous } \\
\text { p.Leu34Arg }\end{array}$ & $\begin{array}{l}\text { Homozygous } \\
\text { p.Arg498* }\end{array}$ & $\begin{array}{l}\text { Homozygous } \\
\text { p.Arg498* }\end{array}$ & $\begin{array}{l}\text { Homozygous } \\
\text { p.Arg498* }\end{array}$ \\
\hline Mental retardation & - & - & - & + \\
\hline Onset age & Third decade & 10 & 13 & 17 \\
\hline $\begin{array}{l}\text { Symptoms at } \\
\text { onset }\end{array}$ & $\begin{array}{l}\text { Rapid eye } \\
\text { movement sleep } \\
\text { behavior disorder }\end{array}$ & $\begin{array}{l}\text { Arm tremor, stiffness, } \\
\text { unsteadiness }\end{array}$ & $\begin{array}{l}\text { Hand tremor, } \\
\text { slowness, } \\
\text { unsteadiness }\end{array}$ & Slowness, tremor \\
\hline Parkinson signs & $\begin{array}{l}\text { Bradykinesia, } \\
\text { rigidity, postural } \\
\text { instability, resting } \\
\text { tremor }\end{array}$ & $\begin{array}{l}\text { Bradykinesia, rigidity, } \\
\text { postural instability, } \\
\text { resting tremor }\end{array}$ & $\begin{array}{l}\text { Bradykinesia, rigidity, } \\
\text { postural instability, } \\
\text { resting tremor }\end{array}$ & $\begin{array}{l}\text { Bradykinesia, } \\
\text { rigidity, resting } \\
\text { tremor }\end{array}$ \\
\hline Pyramidal signs & - & + & + & + \\
\hline $\begin{array}{l}\text { Additional } \\
\text { symptoms }\end{array}$ & $\begin{array}{l}\text { Dysarthria, panic } \\
\text { attacks }\end{array}$ & $\begin{array}{l}\text { Dysphagia, dystonia, } \\
\text { incontinence }\end{array}$ & $\begin{array}{l}\text { Dysphagia, dystonia, } \\
\text { upgaze limitation }\end{array}$ & $\begin{array}{l}\text { Behavioral } \\
\text { disturbances }\end{array}$ \\
\hline $\begin{array}{l}\text { Response to L- } \\
\text { Dopa }\end{array}$ & + & + & + & + \\
\hline $\begin{array}{l}\text { Side-effetcs of L- } \\
\text { Dopa }\end{array}$ & - & $\begin{array}{l}\text { Dyskinesias, } \\
\text { behavioral } \\
\text { disturbances }\end{array}$ & $\begin{array}{l}\text { Dyskinesias, } \\
\text { behavioral } \\
\text { disturbances }\end{array}$ & $\begin{array}{l}\text { Dyskinesias, } \\
\text { behavioral } \\
\text { disturbances }\end{array}$ \\
\hline Brain MRI & Normal & Unremarkable & Unremarkable & Diffuse atrophy \\
\hline
\end{tabular}




\title{
Curriculum Vitae
}

\author{
SIV VINGILL \\ Max Planck Institute of Experimental Medicine \\ Lab of Cellular and Molecular Neurobiology \\ Hermann-Rein-Straße 3 \\ 37075 Göttingen \\ Germany \\ +49 (0)176-70294160 \\ vingill@em.mpg.de, siv.vingill@gmail.com
}

\section{EDUCATION}

2012-present PhD Neuroscience

Georg-August University, Göttingen, Germany

MSc/PhD program: International Max Planck Research School of

Neurosciences

PhD thesis: "Characterization of FBXO7 (PARK15) knockout mice modeling Parkinsonian-Pyramidal Syndrome ", Dr. Judith Stegmüller, Max Planck Institute of Experimental Medicine, Göttingen, Germany

2010-2012 MSc Neuroscience

Georg-August University, Göttingen, Germany

MSc/PhD program: International Max Planck Research School of Neurosciences

Master's thesis: "Biochemical and functional analysis of F-box only protein 7 (FBXO7) in neurons", Dr. Judith Stegmüller, Max Planck Institute of Experimental Medicine, Göttingen, Germany

Aggregate: A (Excellent)

2006-2009 BSc Molecular Biology

University of Oslo, Oslo, Norway

\section{RESEARCH EXPERIENCE}

2011-present Research fellow

Lab of Dr. Judith Stegmueller

Max-Planck Institute of Experimental Medicine

Responsibilties included: Establishing behavioral techniques.

Maintenance of transgenic mouse colony. Supervision of lab-rotation students. Teaching master courses.

2011 Lab rotation projects:

"Verification of gene-expression in the hearing organ of Drosophila melanogaster" Prof. Dr. Martin Göpfert, Cellular neurobiology, Georg August University, Göttingen, Germany 
"Movement of sytlV and BDNF vesicles upon activation of synaptic sites/ The distribution of sytXVII in astrocytes and neuronal cultures", Dr. Camin Dean, Trans-synaptic signalling, European Neuroscience Institute, Göttingen, Germany

"Characterization of FBXO7 mutations involved in Schizophrenia and Parkinson's disorders" Dr. Judith Stegmüller, Max Planck Institute of Experimental Medicine, Göttingen, Germany

\section{Techniques:}

Animal work: Behavioral analysis of mice, pharmacological treatment of mice, mouse genetics, immunohistochemistry, striatal dissection, stereological quantification of neurons and in situ hybridization

Cell culture: Primary neuronal culture, immortalized cell culture, neuronal transfection, RNA interference and immunocytochemistry

Bio-chemical: Proteasome activity assay, co-immunoprecipitation analysis, subcellular fractionation, molecular cloning, protein expression, quantitative RT-pcr and immunoblotting

Microscopy: Fluroescence, bright-field and confocal microscopy of cells and sections and fluorescent live cell imaging

AWARDS AND QUALIFICATIONS

2014

FELASA Category B, University Medical Center, Göttingen

2013-2016 Excellence Stipend, Göttingen Graduate School for Neurosciences, Biophysics and Molecular Biosciences

\section{EXTRA-CURRICULAR ACTIVITIES}

2014-2015 PhD Student Representative, Göttingen Graduate School for Neurosciences, Biophysics and Molecular Biosciences

2013-2016 PhD Student Representative, International Max Planck Research School of Neurosciences

2012-2013 Co-organizer of International Conference: Neurizons 2013, Göttingen, Germany

2012-2013 PhD Student Representative, Max Planck Institute of Experimental Medicine, Göttingen, Germany

2008-2009 Student Representative, Faculty of Biomolecular Sciences, University of Oslo, Oslo, Norway

2007-2009 Voluntary after-school science teacher, Red Cross, Oslo 


\section{PUBLICATIONS, TALKS AND POSTERS}

Dean C, Liu H, Staudt T, Stahlberg M, Vingill S, Bueckers J, Kamin D, Engelhardt J, Jackson M, Hell S, Chapman E (2012) Distinct Subsets of Syt-IV/BDNF Vesicles Are Sorted to Axons versus Dendrites and Recruited to Synapses by Activity. J Neurosci 32(16):5398-5413

Talk: "The role of the E3 ubiquitin ligase FBXO7-SCF in Juvenile Parkinsonism", 2012, Evaluation of IMPRS Neurosciences, Göttingen, Germany

Poster: "Loss of FBXO7 in mice mimics Parkinsonian-Pyramidal symptoms seen in PARK15 patients", Neurizons 2015, Göttingen, Germany

Poster: "Loss of F-box only protein 7 triggers early-onset motor dysfunction in mice", FENS 2014, Milan, Italy 